\title{
Noninvasive reconstruction of cardiac electrical activity
}

Citation for published version (APA):

Cluitmans, M. J. M. (2016). Noninvasive reconstruction of cardiac electrical activity: Mathematical innovation, in vivo validation and human application. [Doctoral Thesis, Maastricht University]. Maastricht University. https://doi.org/10.26481/dis.20160929mc

Document status and date:

Published: 01/01/2016

DOI:

10.26481/dis.20160929mc

Document Version:

Publisher's PDF, also known as Version of record

\section{Please check the document version of this publication:}

- A submitted manuscript is the version of the article upon submission and before peer-review. There can be important differences between the submitted version and the official published version of record.

People interested in the research are advised to contact the author for the final version of the publication, or visit the DOI to the publisher's website.

- The final author version and the galley proof are versions of the publication after peer review.

- The final published version features the final layout of the paper including the volume, issue and page numbers.

Link to publication

\footnotetext{
General rights rights.

- You may freely distribute the URL identifying the publication in the public portal. please follow below link for the End User Agreement:

www.umlib.nl/taverne-license

Take down policy

If you believe that this document breaches copyright please contact us at:

repository@maastrichtuniversity.nl

providing details and we will investigate your claim.
}

Copyright and moral rights for the publications made accessible in the public portal are retained by the authors and/or other copyright owners and it is a condition of accessing publications that users recognise and abide by the legal requirements associated with these

- Users may download and print one copy of any publication from the public portal for the purpose of private study or research.

- You may not further distribute the material or use it for any profit-making activity or commercial gain

If the publication is distributed under the terms of Article $25 \mathrm{fa}$ of the Dutch Copyright Act, indicated by the "Taverne" license above, 


\section{Noninvasive reconstruction of cardiac electrical activity}

Mathematical innovation, in vivo validation and human application

Matthijs Cluitmans
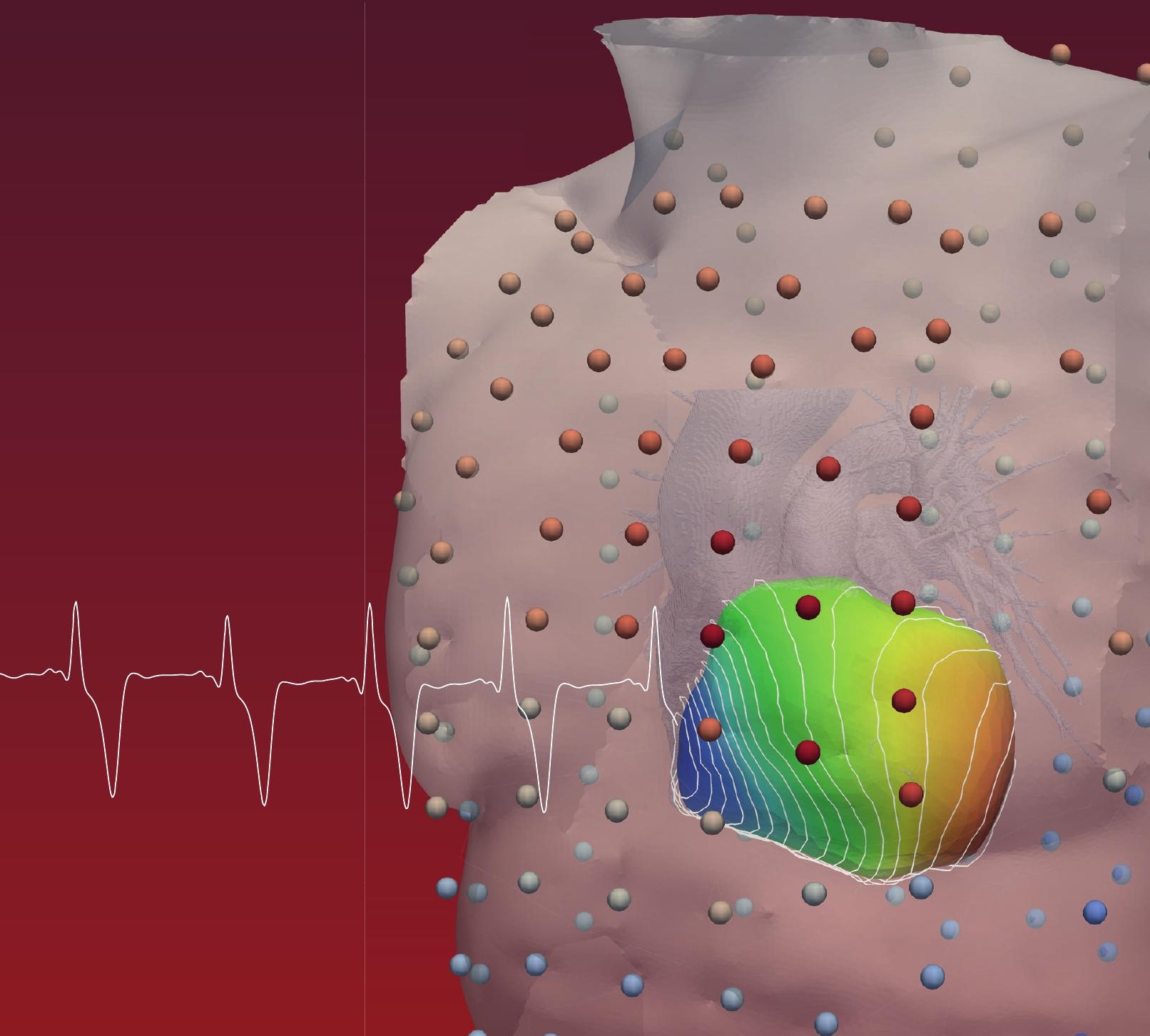
Noninvasive reconstruction of cardiac electrical activity

Mathematical innovation, in vivo validation and human application

\section{Matthijs Cluitmans}

$$
2016
$$




\section{Noninvasive reconstruction of cardiac electrical activity Mathematical innovation, in vivo validation and human application}

\section{PROEFSCHRIFT}

ter verkrijging van de graad van doctor

aan de Universiteit Maastricht, op gezag van de Rector Magnificus,

Prof.dr. Rianne M. Letschert, volgens het besluit van het College van Decanen, in het openbaar te verdedigen op donderdag 29 september 2016 om 16:00 uur

door

Matthijs Joseph Maria Cluitmans 


\section{Promotores}

Prof. dr. P.G.A. Volders

Prof. dr. ir. R.L.M. Peeters

Prof. dr. R.L. Westra (Universiteit Hasselt, België)

\section{Beoordelingscommissie}

Prof. dr. H.J.G.M. Crijns (voorzitter)

Prof. dr. O. Dössel (Karlsruhe Institute of Technology, Duitsland)

Prof. dr. E. Formisano

Prof. dr. Y. Rudy (Washington University in St Louis, VS)

Prof. dr. H.J.J. Wellens

Prof. dr. J.E. Wildberger

Financial support by the Dutch Heart Foundation for the publication of this thesis is gratefully acknowledged. The research described in this thesis was supported by a grant of the Dutch Heart Foundation ( $\mathrm{NHS}_{2007} \mathrm{~T}_{51}$ ). Additional support by the transnationale Universiteit Limburg ( $\mathrm{tUL}$ project $\mathrm{NS}_{1} \mathrm{~b}$ ) is gratefully acknowledged as well.

(C) Matthijs Cluitmans, 2016

ISBN 978-94-6159-594-2 
Voor mijn ouders 


\section{Contents}

1 INTRODUCTION 1

2 ECGI: A REVIEW ON CURRENT METHODS, APPLICATIONS AND CHALLENGES 19

$3 \quad$ In VIVO VALIDATION OF ECGI 36

4 Physiology-based regularization in ECGI $\quad 62$

5 WAVELET-BASED REgUlarization IN ECGI $\quad \mathbf{8 1}$

6 INTEGRATION OF ECGI OF REPOLARIZATION WITH CELLULAR MODELING IN A PATIENT WITH VF 95

7 General discussion $\quad 107$

8 SumMary

9 SAmenVATting $\quad 147$

$\begin{array}{lr}10 \text { VAlorisation } & 153\end{array}$

11 ACKNowledgements / Dankwoord $\quad 158$

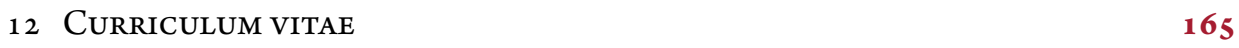



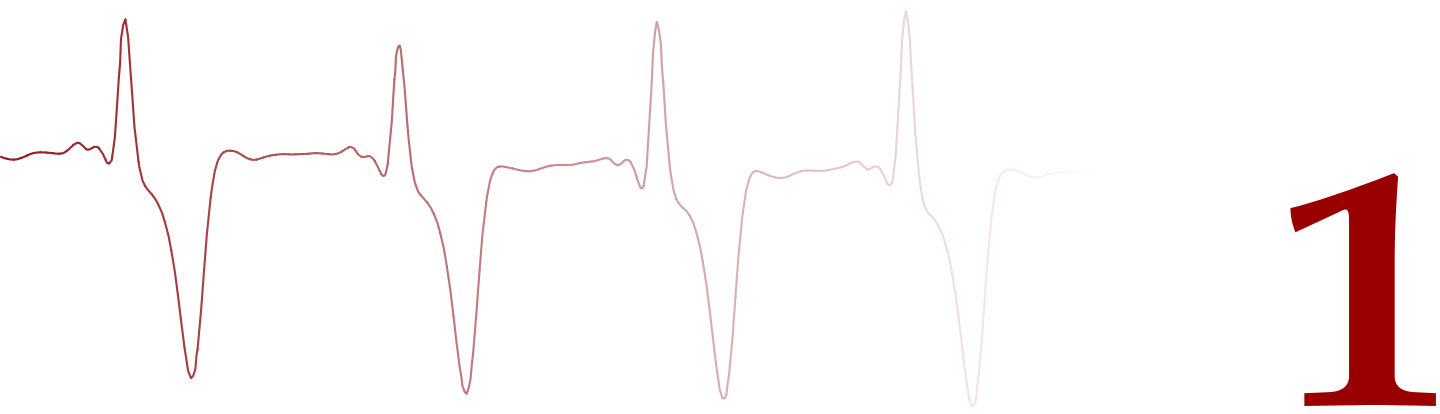

\section{Introduction}

\section{Contents}

1.1 The origin of electrical heart activity . . . . . . . . . 4

1.2 Arrhythmogenesis . . . . . . . . . . . . . . 6

1.3 A short history of the ECG . . . . . . . . . . 8

1.4 Development of electrocardiographic imaging . . . . . . 10

1.5 Implementation of ECGI in Maastricht $\ldots \ldots \ldots \ldots$

1.6 Challenges and goals . . . . . . . . . . . . . 16

1.7 Structure of this thesis . . . . . . . . . . . . . . 18 
The electrocardiogram (ECG) is a well-established, validated, patient-friendly, quick, reproducible and cheap tool to assess the electrical activation and recovery of the heart as projected on the body surface. It provides valuable information about the rhythm of the heart, and about rhythm disorders.

Due to the attenuation and dispersion of electromagnetic propagation in the torso, the ECG is a superimposed and 'smeared' representation of the actual cardiac electrical activity. Thus, it lacks the capacity to assess electrical activity at high resolution at the level of the heart muscle (myocardium) at high resolution. Studies to evaluate the electrical activity directly on the heart surface, not body surface, require invasive approaches and are thus are limited by practical and ethical considerations. In 1970, Durrer et al reported on direct epicardial and intramural recordings of electrical activation from explanted hearts of humans who died in the absence of cardiac disease, see Figure 1.1. [1] It allowed, for the first time, to investigate normal electrical activity directly at the level of the human heart. To date, this landmark study remains a frequently-used source of information, as is evident from the numerous times it is cited in subsequent literature reports (Figure 1.2), indicating how difficult but valuable it is to image the electrical state of the heart with this level of detail.

In this thesis, I investigate electrocardiographic imaging (ECGI), a modality that noninvasively images electrical activation and recovery directly at the heart surface. Its
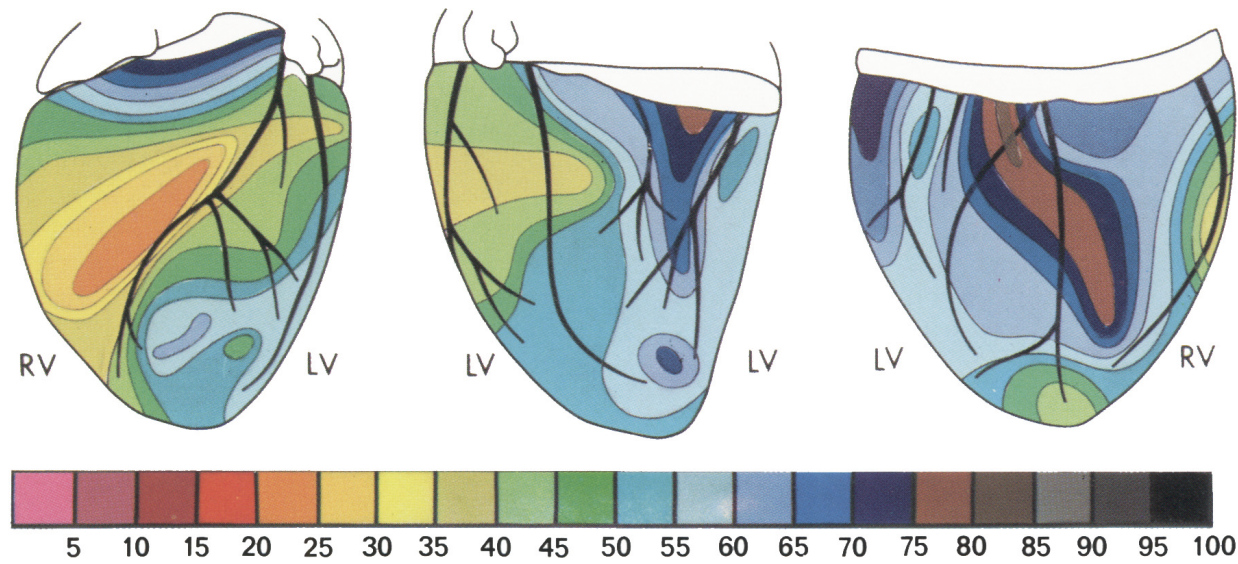

Figure 1.1: Electrical activation of an explanted human heart, as recorded by Durrer et al in in the 1960s [1] on explanted hearts. Recorded activation time (in ms) from the same heart in anterior (left), left lateral (middle) and posterior view (right). RV = right ventricle; $\mathrm{LV}=$ left ventricle. 


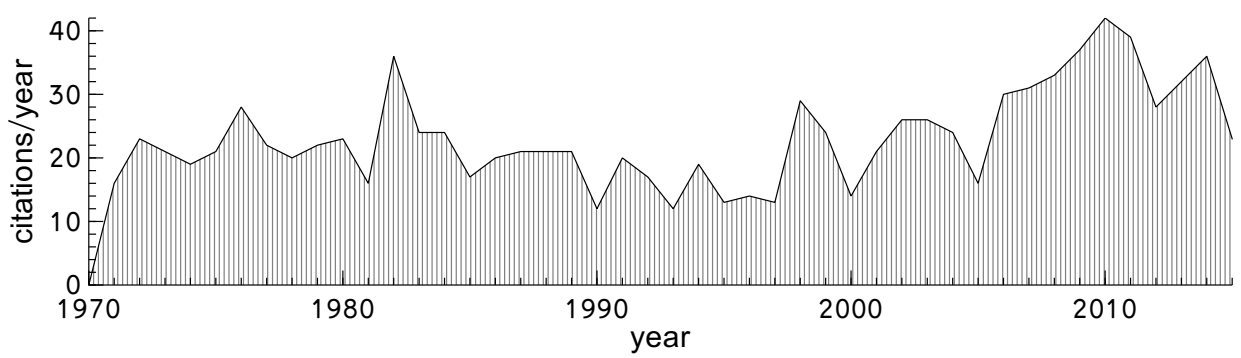

Figure 1.2: Yearly citations of the landmark study by Durrer et al [1], according to Web of Science [2], adding to a total of more than 1,000 citations.

patient-specific approach can provide new insights in cardiac electrophysiology and arrhythmias and it can guide therapy.

In this introductory chapter, I discuss the basics of cardiac electrical activity, as well as the history and development of the ECG and ECGI. Finally, the scientific goals and general layout of this thesis are described.

\subsection{THE ORIGIN OF ELECTRICAL HEART ACTIVITY}

From a mechanical point of view, the heart is a pump and consists of two separate, but interconnected systems: the right system pumps blood to the lungs, and then to the left system, which pumps blood to the rest of the body. Each system consists of two chambers: an atrium and a ventricle. The ventricles are supplied with blood by the atria. Arrhythmias in the ventricles can be directly life-threatening if they prevent adequate pumping capacity.

From an electrical point of view, contraction of the heart muscle is initiated by an electrical impulse that travels through the tissue as an excitation wave front (Figure 1.3, left). This activation wave front originates from the sinus node in the right atrium, and is then conducted to the atrioventricular (AV) node. During this time interval, the atria are electrically activated and contract, pumping blood to the ventricles. After a necessary delay to allow time for the filling of the ventricles, the AV node activates the ventricular conduction system, consisting of the His bundle, the left and right bundle branches and ultimately the Purkinje fibers, which spread throughout the ventricular muscle. The quick conduction system then ensures almost simultaneous electrical activation and subsequent mechanical contraction of the ventricular myocardium, resulting in efficient pumping. 


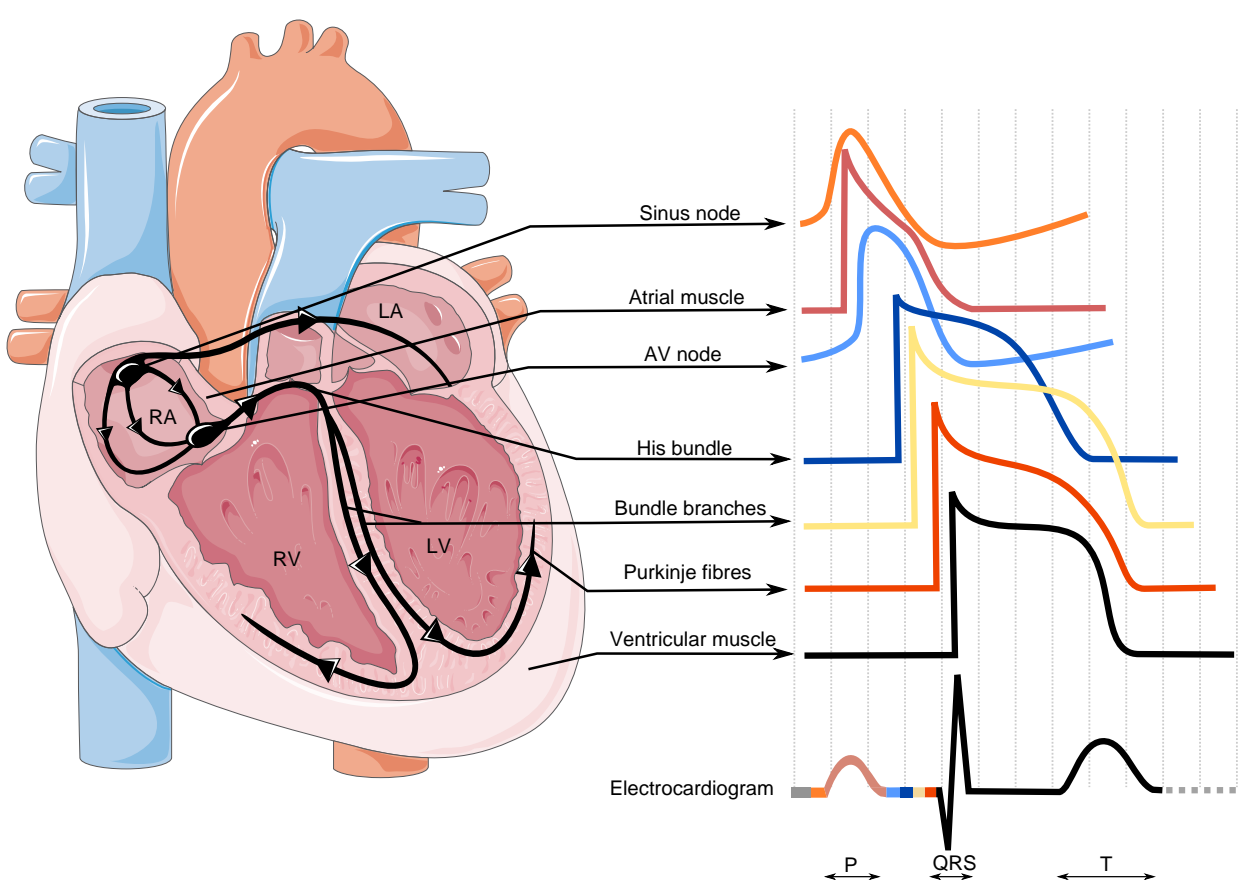

Figure 1.3: Left: the heart and its electrical conduction system. Right: the heterogeneous shapes of action potentials throughout the heart, which at the body-surface can be recorded as an electrocardiogram. RA: right atrium; LA: left atrium; LV: left ventricle; RV: right ventricle; $\mathrm{AV}$ : atrioventricular.

The electrical impulse that travels through the heart is formed by the individual activation of millions of cardiomyocytes (heart muscle cells). Activation of a cardiomyocyte generates a typical change in the cellular membrane potential, which is called the action potential (AP). The shape of the AP differs for the different types of cardiomyocytes (Figure 1.3, right). The electromagnetic field that results from all APs can be recorded as the ECG on the body-surface. The P wave on the ECG is the result of electrical activation of atrial cardiomyocytes, the QRS complex results from electrical activation of the ventricular muscle, and the $\mathrm{T}$ wave is the result of the electrical recovery (repolarization) of ventricular muscle.

APs reflect the voltage changes over the cell membrane of a cardiomyocyte, resulting from changes in the intracellular or extracellular concentrations of charged atoms (ions), notably sodium $\left(\mathrm{Na}^{+}\right)$, potassium $\left(\mathrm{K}^{+}\right)$, and calcium $\left(\mathrm{Ca}^{2+}\right)$ ions. For example, in a ventricular myocyte at rest, the intracellular concentration of $\mathrm{Na}^{+}$is approximately 14 times lower than the extracellular concentration, the intracellular concentration of $\mathrm{K}^{+}$is approximately 40 times higher than extracellular, and the intracellular concentration of 
calcium ions $\mathrm{Ca}^{2+}$ 20,000 lower than extracellular. [3]

APs are the result of a complex interplay between ion channels, pumps and exchangers in a cardiomyocyte, which control the inflow and outflow of ions through the cell membrane (Figure 1.4, top). The shape of the AP reflects different phases of opening and closing of different ion-channel types, which results in ion currents (Figure 1.4, bottom). The upstroke of the AP (phase o) is initiated either when ions enter the cell from neighboring cardiomyocytes, or when a nearby electromagnetic field (from a depolarization wave front) creates a shift in intracellular pooling of ions towards or away from the membrane surface. [3] This results in a depolarization of the membrane, which, if a threshold is reached, is followed by opening of voltage-dependent $\mathrm{Na}^{+}$channels and inflow of $\mathrm{Na}^{+}$, resulting in a rapid full depolarization. Subsequently, the $\mathrm{Na}^{+}$channels inactivate and $\mathrm{K}^{+}$and $\mathrm{Ca}^{2+}$ channels open. This new balance results in early repolarization (phase 1 ) and the plateau phase (phase 2 ). The opening of L-type $\mathrm{Ca}^{2+}$ channels on the membrane results in a small $\mathrm{Ca}^{2+}$ influx that rapidly triggers a much larger $\mathrm{Ca}^{2+}$ release from the sarcoplasmic reticulum, a process termed $\mathrm{Ca}^{2+}$-induced $\mathrm{Ca}^{2+}$-release. During the relatively long plateau phase, the large intracellular $\mathrm{Ca}^{2+}$ concentrations promote binding of calcium to the troponin complex, thereby activating contraction in the sarcomere, and thus contraction of the cell. After repolarization of the membrane potential to its resting state by time-dependent activation of $\mathrm{K}^{+}$channels, intracellular ion concentrations are restored to normal values by the $\mathrm{Na}^{+}-\mathrm{K}^{+}$pump, the $\mathrm{Na}^{+}-\mathrm{Ca}^{2+}$ exchanger and the sarcoplasmic reticulum $\mathrm{Ca}^{2+}$ pump.

Thus, the electrical balance that results from the ion currents in cardiomyocytes throughout the heart is essential for the rhythmic mechanical contraction of the heart. A disbalance in the electrical activation can produce arrhythmia, which can severely disturb the normal function of the heart.

\subsection{ARRHYTHMOGENESIS}

Mechanisms of cardiac arrhythmias are usually divided in two main categories: abnormal impulse formation (automaticity or triggered activity) and abnormal impulse conduction (reentry). Combinations of these probably occur often. [4] This classification does not reflect underlying causes of arrhythmogenesis, e.g., genetic mutations to ion channels or regional ischemia. Diagnosis of underlying arrhythmia mechanisms can be of great help in guiding treatment strategy. 

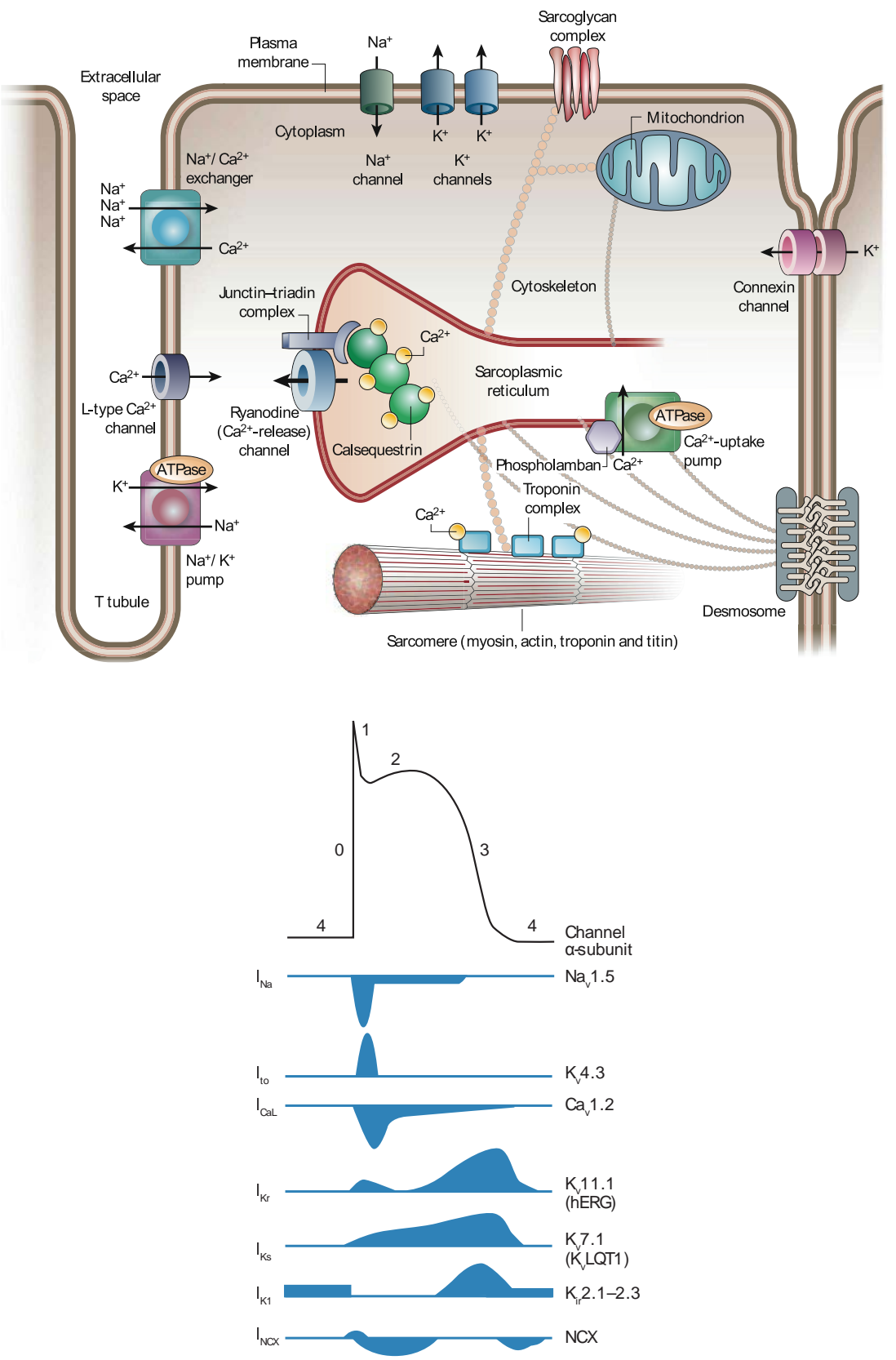

Figure 1.4: Top: Model of a ventricular cardiomyocyte. Illustrated are the ion channels involved in cardiac excitation, and the intracellular structures involved in contraction. Bottom: A schematic representation of the cardiac action potential with the major contributing cardiac ion channel currents. 
Abnormal automaticity Spontaneous impulse formation (termed automaticity) is a normal property of cardiomyocytes from the sinus node. These cells spontaneously produce a rhythmic depolarization that is not initiated by other APs, allowing the development of a wave front that activates all other cardiomyocytes. Non-sinus-node cardiomyocytes also show some automaticity, but normally at such slow rates that it has no physiological role. If the sinus fails, the automaticity of other cardiomyocytes of the conduction system induces a life-saving escape rhythm. Under specific conditions, cardiomyocytes can show enhanced normal automaticity or abnormal automaticity, which may occur in cells that normally do not exhibit automatic properties. Such automaticity can be induced by drugs, cardiac disease, changes in extracellular potassium, or sympathetic nervous stimulation.

TRIGgered ACTIVITY Triggered activity is spontaneous impulse formation initiated by an afterdepolarization. Afterdepolarizations are depolarizing oscillations in membrane potentials that occur during an AP (termed early afterdepolarizations) or after full repolarization (termed delayed afterdepolarization). If the afterdepolarization reaches the threshold of AP excitation, a new (so-called triggered) AP is generated. Unlike automaticity, triggered activity is not self-generating, but a response to a preceding AP. [4]

AbNoRmal CONDUCTION AND REENTRY During most moments of the AP, the cell is not sensitive to new stimuli, i.e., it is not excitable. This so-called refractoriness protects the heart from inadvertent electrical stimulation, thus permitting relaxation of cardiac muscle before subsequent reactivation. Reentry occurs when a propagating AP wavefront continues to reexcite cardiac tissue in a circuitry with abnormal conduction properties and/or refractoriness. Normally, activation wavefronts vanish spontaneously after all cardiac tissue has been activated due to the long duration of refractoriness of the heart compared with the duration of the excitation period. [4] In pathological circumstances, shortening of refractoriness or slowing of conduction velocity ensures that the wave front can continue to excite tissue, resulting in a reentry tachycardia. Reentry can be initiated when a wave front blocks in parts of the cardiac tissue, or circulates around zones of block, and reactivates tissue that has recovered in the mean time.

\subsection{A SHORT HISTORY OF THE ECG}

To diagnose and treat cardiac arrhythmias, assessment of the electrical functioning of the heart is essential. For this purpose, the ECG has proven to be extremely valuable as 


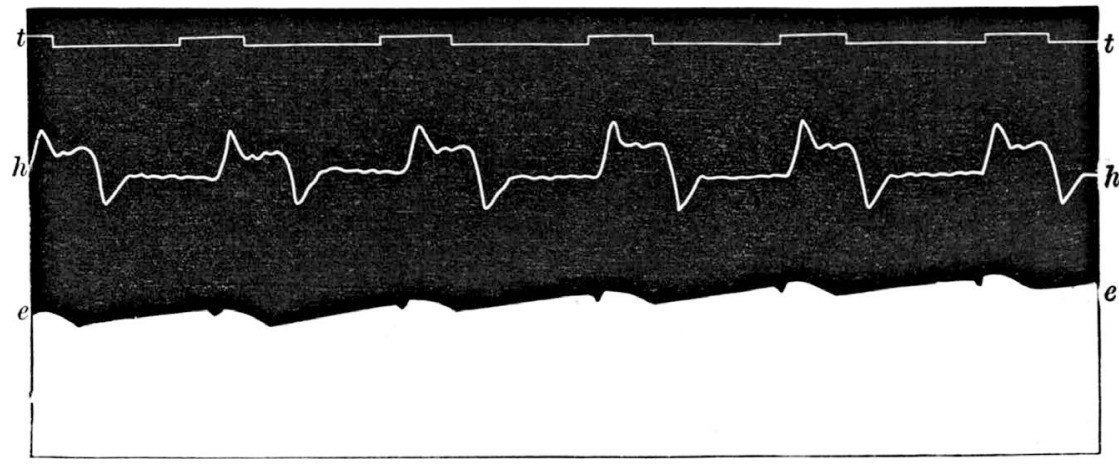

Figure 1.5: First human ECG, by Waller [8]. The line $t$ represents time in seconds, $h$ the chest-wall movement due to the heart contraction (an 'apex cardiogram') and $e$ the electrocardiogram as recorded by movement of a mercury column connected with two electrodes to the front and back of the chest.

a clinical tool for over a century. [5] Some insight in the history of the ECG helps to put the current state of the art of this thesis in perspective.

The first notions of electrical activity in living organisms ('animal electricity') appeared in the 18 th century. In 1791, Galvani showed that electrical stimuli yield contraction of the heart muscle in a frog. [6] Matteucci showed in 1838 that an electric current accompanies each heart beat. [7] He detected electrical activity using an instrument called 'rheoscopic frog' or 'frog galvanoscope', which is made of the leg muscle of a frog. Matteucci showed that the frog leg, when connected to a heart, would twitch with the rhythm of the beating heart, indicating the presence of a rhythmic electrical current. [7]

In the second half of the 19 th century, the electrical activity of the heart was recorded by different investigators. Although the quality of the recordings was very poor, two different phases could be distinguished during a heart beat, now known to be corresponding to ventricular activation and recovery. In 1887 , the British physiologist Waller published the first human ECG [8], see Figure 1.5. In 1893, inspired by Waller, the Dutch physician Einthoven introduced the term 'electrocardiogram' [9].

In 1895 Einthoven, combining an improved recording device and a correction formula, constructed an ECG that resembles modern day recordings [10], Figure 1.6. When correcting the original recording (in which he annotated the deflections by A, B, C and D) he used the first characters of the second half of the alphabet and arrived at the modernday notation of the $\mathrm{P}, \mathrm{Q}, \mathrm{R}, \mathrm{S}$ and $\mathrm{T}$ deflections. He improved ECG recording quality and introduced the 'Einthoven triangle', in which three ECGs are recorded from three 


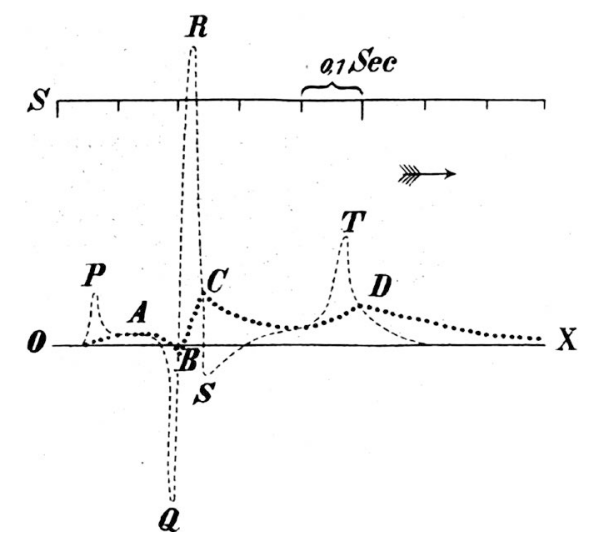

Figure 1.6: Einthoven's corrected human ECG [10]. The original recording (thick, dotted line; $A, B, C, D$ ) is corrected to arrive at a modern-day recording with $P, Q R S$ and $T$ waves (thin, dashed line).

electrodes: one at the right arm, one at the left arm, and one at the left leg, Figure 1.7. These recordings are called the limb (or extremity) leads, and annotated I, II and III.

Einthoven was also amongst the first to show the clinical applications of the ECG, by presenting normal and abnormal electrocardiograms with left and right ventricular hypertrophy, left and right atrial hypertrophy, the U wave, notching of the QRS complex, ventricular premature beats, bigeminy, atrial flutter and complete heart block [ 11 . In 1924, he was awarded the Nobel prize for his 'discovery of the mechanisms of the electrocardiogram'.

In 1938 , the position of the precordial leads was standardized. [12] These leads, annotated $V_{1}$ to V6, are added to the three leads of Einthoven and are placed at the front and left side of the thorax. In 1942, the augmented limb leads aVR, aVL and aVF were defined as different derivations of the existing limb electrodes. The combination of Einthoven's three limb leads (I, II, III), the augmented limb leads from the same three electrodes (aVR, aVL, aVF), and the six chest leads from the precordial electrodes ( $\left.\mathrm{V}_{1}-\mathrm{V} 6\right)$, thus in total 9 electrodes, gives rise to the 12-lead ECG that is used nowadays, Figure 1.8.

\subsection{DEVELOPMENT OF ELECTROCARDIOGRAPHIC IMAGING}

Adding more electrodes is a trend in the history of the ECG, Figure 1.9. Many additional and alternative electrode positions have been investigated. During the 1960s, researchers 


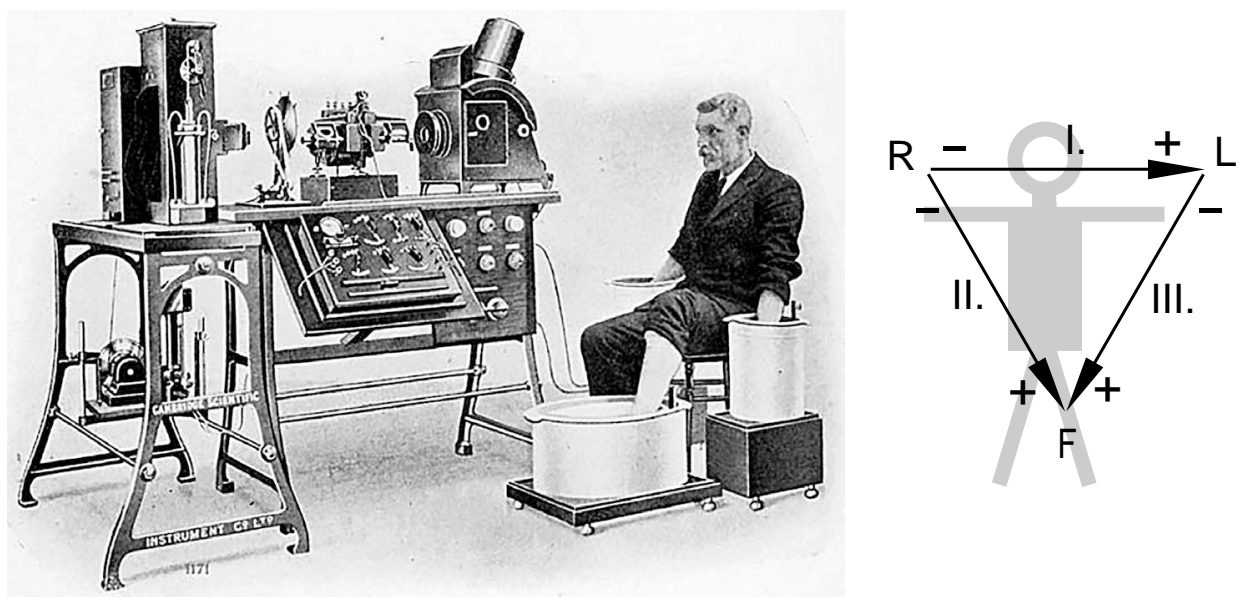

Figure 1.7: Left: an early commercial ECG machine, recording a three-lead ECG from the two arms and one leg by submersion of those limbs in buckets with electrolyte solution. This would capture the electrical activity of the heart in three directions, known as lead I, II and III of the Einthoven triangle (right).

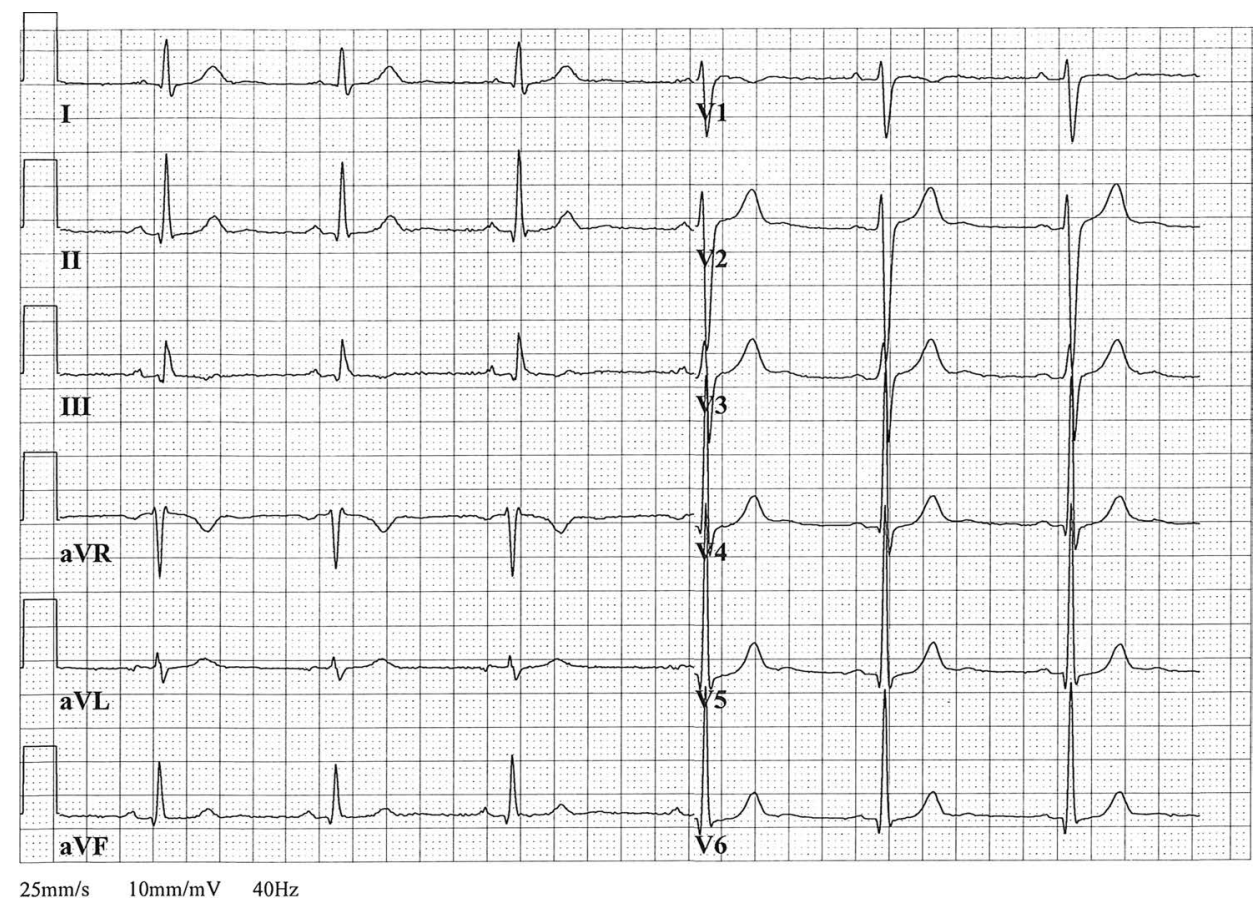

Figure 1.8: The author's (normal) 12-lead ECG. 

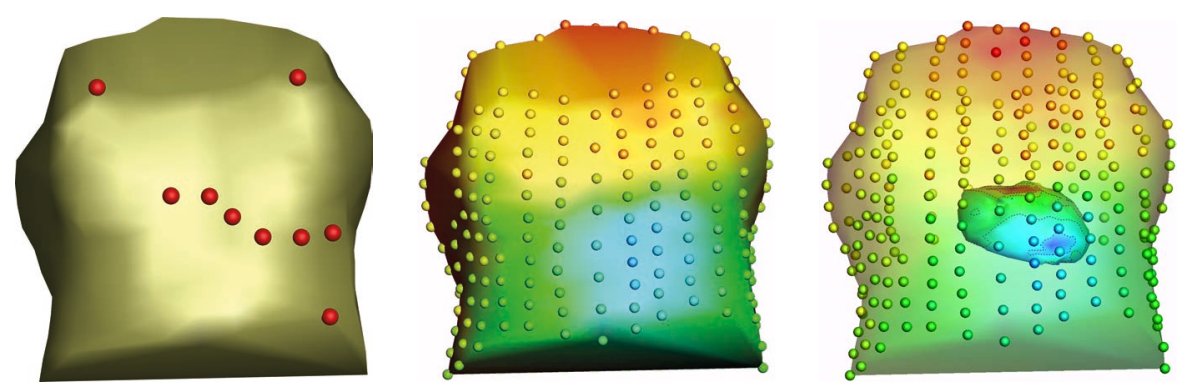

Figure 1.9: Digitized torso with the three limb electrodes and six precordial electrodes of the default 12-lead ECG (left), a body-surface potential map (BSPM) recorded with 256 electrodes on the torso (middle), and the geometrical relation between heart-surface potentials and the body-surface potentials (right).

started using tens to hundreds of body-surface recordings, yielding body-surface potential maps (BSPMs). However, interpretation of these BSPMs was not straightforward and clinical applications remained very limited. [13] Nevertheless, it seemed logical that a larger number of electrodes would allow a more thorough understanding of the electrical activity of the heart, especially if this extensive recording would allow to reconstruct the electrical activity directly on the heart surface.

So called 'forward models' describe the propagation of electromagnetic activity from heart surface to body surface (Figure 1.10), and are based on the laws of electromagnetism. An inverse model is based on a forward model with known 'output' and unknown 'source'; essentially it aims at reversing the natural electromagnetic relationship between heart and body surface. The problem of reconstructing electrical activity at the level of the heart tissue from recorded body-surface potentials is called the inverse problem of electrocardiography. Methods that achieve this have commonly been called electrocardiographic imaging (ECGI).

To solve the inverse problem, it is essential to have a good forward model. If the electrical activity of the heart is known, the laws of electromagnetism can be used to compute the corresponding electrical potentials at the body surface. The propagation is dependent on conductivity of the intrathoracic organs and tissue, and their geometrical relation. The geometry can be obtained from computed tomography (CT) or magnetic resonance imaging (MRI) scans. Usually, the torso is then modeled as a piecewise homogeneous medium, i.e., a medium in which there are areas of distinct conductivities. Combined with the assumed properties of the human torso (e.g., absence of capacitive and inductive effects), the intrathoracic electromagnetic fields can then be described quasi- 


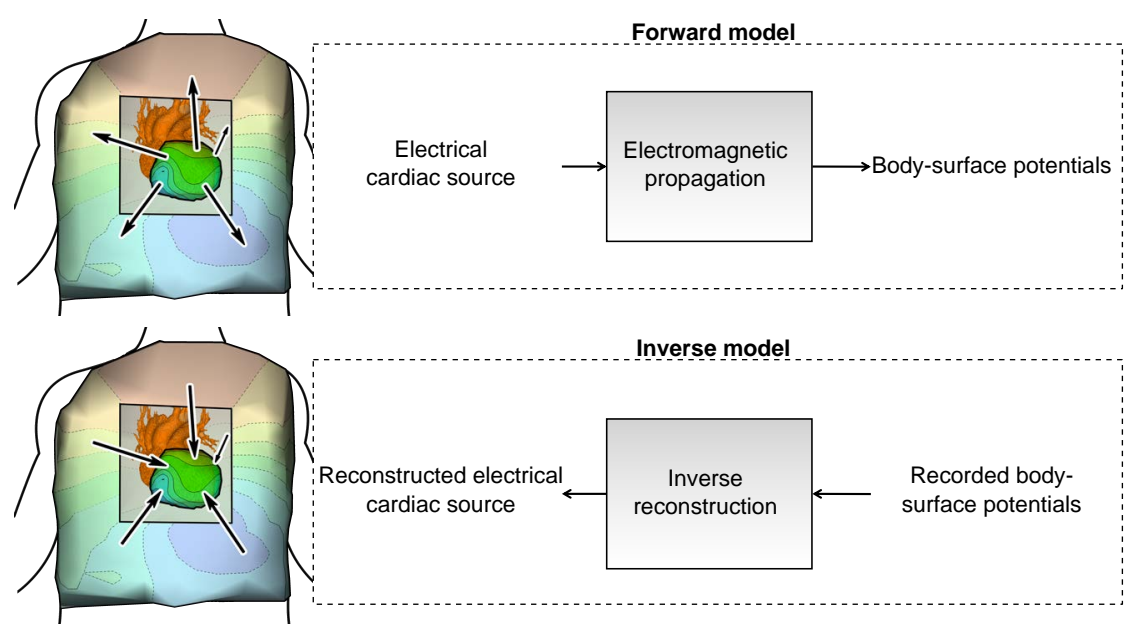

Figure 1.10: Schematic representation of forward and inverse models. A forward model describes the propagation of electromagnetic activity from the heart to the body surface; an inverse model reverses that relation, allowing for noninvasive reconstruction of electrical heart activity from measured body-surface potentials. More details in Chapter 2.

statically. [14] This means that, although potentials change over time, their distribution at any time instant can be computed by the electrostatic field equations, independent of time. [14]

An essential step in forward and inverse modeling is the source description, i.e., the model for the electrical activity of the heart. Currently, the two most commonly applied source descriptions are the potential-based formulation, and the wave-front based formulation. The potential-based formulation reconstructs potentials at the heart surface, whereas the wave-front formulation reconstructs local activation and recovery times.

The potential-based formulation is based on the electrostatic relation between potentials on the body surface, and the potentials on a (virtual) closed surface that contains all electrical sources within the thorax. [14] A logical choice for such a surface seems to be the epicardium (outer heart surface), as it contains all significant electrical sources of the heart, Figure 1.11a. If one is only interested in the ventricular activity and not atrial activity, this virtual surface can be capped at the base of the heart. An alternative formulation of the potential-based source model also incorporates the endocardium (inner heart surface), Figure $1.11 \mathrm{~b}$. This formulation has the added advantage that it would also reconstruct endocardial electrical activity. However, it has not been extensively investigated or validated in vivo, or in clinical studies.

The wave-front source model is based on the reconstruction of action potentials. For 


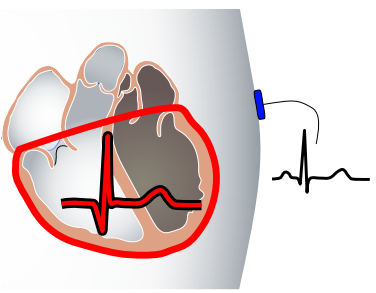

(a) Epicardial potentials

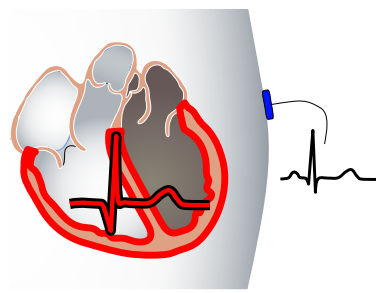

(b) Epi-/endocardial potentials

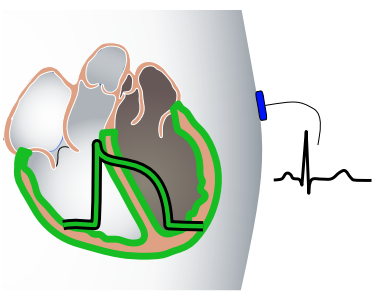

(c) Equivalent double layer

Figure 1.11: Different source models for electrical activity of the heart. The recording electrode on the body surface (blue) records electrical potentials. The heart activity that is the origin of the body-surface potentials can be described with different source models, either in terms of potentials (red), or in terms of an equivalent double layer (green).

this purpose, a simple template for APs is used per virtual location on the endocardium and epicardium to form an equivalent double layer (EDL) source model. [15], [16] Although the AP template is too simple to gain insights in cellular mechanisms, its upslope and downslope are representative for local tissue activation and recovery. Thus, the EDL models activation and recovery wave fronts, Figure $1.11 \mathrm{c}$.

The epicardial-potential based formulation is the most studied source model in the inverse problem of electrocardiography. It has been validated in experimental setups and is applied in clinical settings. Therefore, it is also the formulation that was used for implementation of ECGI in this thesis.

\subsection{IMPLEMENTATION OF ECGI in MAASTRICHT}

The first studies on ECGI in Maastricht were performed in 2007 and investigated some theoretical characteristics of the potential-based formulation. [17] The hardware for body-surface potential recordings was acquired in 2009 and was followed by implementation of software for analysis of acquired data.

The current implementation of ECGI is visualized in Figure 1.12. Using the ActiveTwo setup (BioSemi, Amsterdam, the Netherlands), up to 256 body-surface electrodes are attached to a subject's torso (front, back and lateral) and used to record potentials at $2048 \mathrm{~Hz}$. Computed tomography (CT) is used to capture the geometry of the heart and torso. The heart surface is imaged with a cardiac CT scan. The cardiac scan is ECG gated, meaning that the CT slices are obtained during the same phase of the cardiac cycle during consecutive beats. Usually, the heart is scanned in the diastolic (i.e., relaxed) state. Intravenous iodine contrast medium is used to visualize the cardiac 

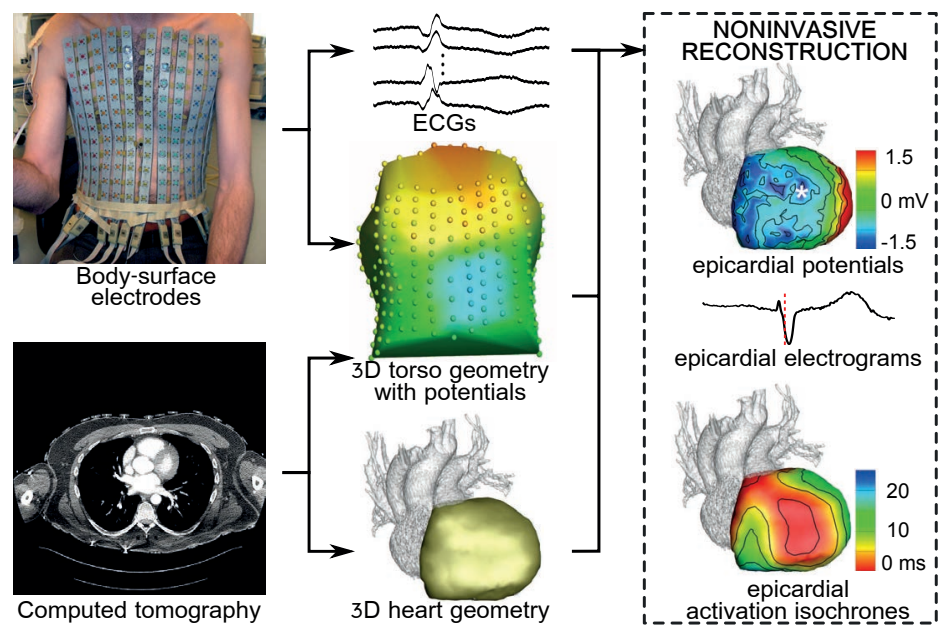

Figure 1.12: Electrocardiographic imaging (ECGI) as implemented in Maastricht. Bodysurface potential recordings and a computed tomography (CT) scan are combined in a torsoheart geometry. Epicardial potentials are reconstructed from the body-surface ECGs and yield local electrograms, activation isochrones and recovery isochrones.

structures. The position of the body-surface electrodes is captured by a thoracic CT scan (without the use of a intravenous contrast agent or ECG gating). In a preclinical study, the CT scanning protocol was optimized to reduce scattering artifacts from the bodysurface electrodes, while minimizing radiation dose, see Figure 1.13.

The torso-heart geometry in the Maastricht ECGI implementation is defined by the position of body-surface electrodes and the ventricular epicardium. These structures are segmented from the cardiac and thoracic CT scan with the publicly available $\mathrm{Seg}_{3} \mathrm{D}$ software (Scientific Computing and Imaging Institute (SCI), Salt Lake City, Utah, USA). [18] The transfer matrix, capturing the geometry and conductivity relation between the heart and body surfaces, is also computed with publicly available SCI software. [19] The potential data and transfer matrix are then processed in custom-made software in Matlab to obtain epicardial potentials. [20], [21] Post-processing of the reconstructed potentials is used to obtain activation and recovery times, to visualize these as isochrones on the epicardial surface, and to localize beat origins. Further software was developed to integrate ECGI-reconstructions in electroanatomical mapping systems that are used during clinical ablation procedures.

The first patient was included in ECGI research in Maastricht in 2011, and since 2014, ECGI has been more routinely applied in the Maastricht University Medical Centre (MUMC+) for scientific and clinical purposes. 


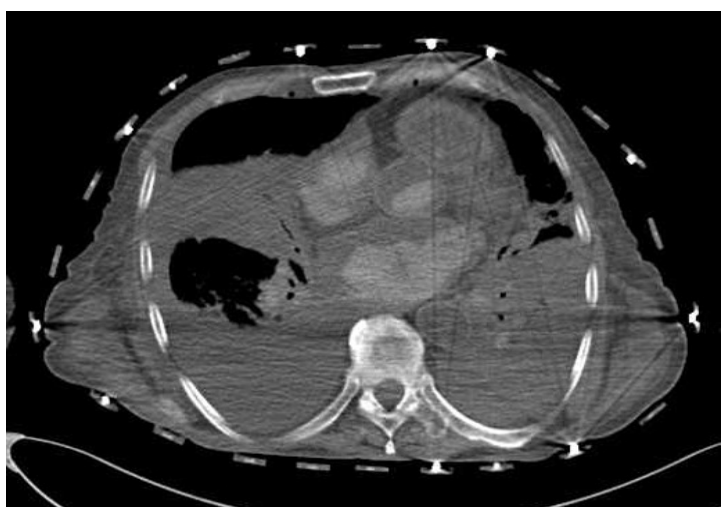

Figure 1.13: CT scan of a human cadaver performed in MUMC+, to investigate the effect of the body-surface electrodes on scan quality. The electrodes result in scattering, which can hinder diagnostic assessment of intrathoracic structures. This scan was used to optimize scan protocols to reduce scattering artifacts due to the electrodes.

\subsection{Challenges AND gOAlS}

The goals of this thesis stem from challenges in implementing, validating, improving and applying ECGI.

IMPLEMENTATION During implementation of ECGI in MUMC+, technical and practical challenges arose. Amongst those were the development of a CT protocol, the determination of the number of body-surface electrodes needed for accurate inverse reconstruction, and the need for methods to estimate activation and recovery times from reconstructed electrograms. Especially when locally reconstructed electrograms are noisy or fractionated, estimation of activation and recovery times can be challenging. We introduce new computational methods to deal with this.

VALIDATION To assess the accuracy of ECGI, it is essential to obtain in vivo recordings of electrical potentials at the heart surface. However, these data are difficult to obtain because of practical and ethical limitations. Previous studies either focused on torso-tank experiments, in which there is a lot of experimental control but limited physiological realism, or on human data, in which invasive electrogram recordings are available only to a very limited extent and obtained at different moments. We hypothesize that the necessary high quality, simultaneous, invasive data can be obtained from animal experiments. These data will help to investigate the spatial accuracy of reconstructed electrograms, the accuracy of activation and recovery times, and the influence of cardiac motion. Insights in these processes will help to bring significant improvements to ECGI. 
Mathematical improvement Due to the attenuation and dispersion of the torso, the potentials on the body-surface are a superimposed and 'smeared' representation of the electrical activity of the heart. Additionally, simplifications and assumptions are necessary to arrive at models that can be used for inverse reconstruction. Consequently, uncertainty at different levels is involved in the inverse reconstruction of electrical potentials at the heart surface. Therefore, to unravel body-surface recordings to their source on the heart surface, mathematical techniques are needed to deal with the involved uncertainty. We postulate that this uncertainty can be reduced if the source models of the electrical heart activity are designed such that they yield an efficient (i.e., sparse) representation of the data.

Clinical and mechanistic application ECGI can be applied for diagnostic purposes, monitoring of treatment, and mechanistic research. Amongst all possible applications, ECGI may help to guide ablation therapy by localizing the origin of abnormal (extrasystolic) beats. Moreover, ECGI allows to investigate the electrical substrate of arrhythmias that cannot be detected on the normal ECG, such as local recovery abnormalities. In combination with translational cellular studies, this can bring new insights in disease mechanisms.

GoALs Thus, the goals of the research presented in this thesis were to implement ECGI at MUMC+, to improve and validate novel ECGI algorithms, to use ECGI to obtain new insights in arrhythmogenesis, and to use ECGI to support ablation therapy in patients. This can be captured by the following research questions:

1. What is the in vivo accuracy of electrograms that are noninvasively reconstructed on the heart surface?

2. How can activation and recovery times be estimated accurately from reconstructed electrograms?

3. How can the origin of a beat be estimated accurately from reconstructed electrograms?

4. What is the influence of cardiac motion on reconstruction accuracy?

5. What is the influence of the number of body-surface potentials on reconstruction accuracy?

6. Can novel cardiac source models that aim for a sparse representation of the electrical activity on the heart surface, improve reconstruction accuracy?

7. Can localization of (abnormal) beat origins by ECGI support ablation therapy in patients? 
8. Can ECGI detect the electrical substrate for cardiac arrhythmias that is not apparent from the 12-lead ECG?

\subsection{STRUCTURE OF THIS THESIS}

These research questions shaped the structure of this thesis. The current status of the ECGI field is reviewed in Chapter 2. We illustrate this with the first clinical case from Maastricht and demonstrate some of the possibilities with ECGI. The Maastricht ECGI implementation is then extensively validated in an animal study in Chapter 3. This study provides insights in factors influencing the accuracy of ECGI, and quantifies, for the first time, the quality of reconstructed activation and recovery isochrones. We investigate the spatial accuracy of electrogram reconstructions, and demonstrate the added benefit of a spatiotemporal approach to reliably reconstruct beat origins, and activation and recovery times. Moreover, we investigate the influence of cardiac motion on reconstruction accuracy.

Novel algorithms to improve ECGI accuracy are introduced in Chapters 4 and $\mathbf{5}$. These methods are based on different representations of the data, in order to obtain a sparser description of the inverse problem that is less sensitive to noise. The physiologybased method (Chapter 4) is inspired by 'physiologically realistic' spatial distributions of potentials, which are simulated with AP models and subsequently used as building blocks for the inverse procedure. The wavelet-based method (Chapter 5 ) uses temporal building blocks to describe the reconstructed potentials more efficiently. Both novel methods increase accuracy of recovery times, which is relevant when investigating the influence of recovery abnormalities on the development of cardiac arrhythmias.

In Chapter 6, the value of ECGI is further illustrated by a clinical case. This chapter also demonstrates the added value of a translational approach, by combining ECGI with computational modeling of the effects of genetic mutations of ion channels. It highlights the role of recovery abnormalities in arrhythmogenesis, and the role of ECGI to detect these abnormalities, even when not visible from the 12-lead ECG.

Finally, Chapter 7 puts the results obtained in this thesis in perspective. This chapter also offers some examples of clinical cases where ECGI was used to guide ablation therapy, and it shows a first example of image integration with MRI. Additionally, we shed some light on practical aspects of ECGI that were not discussed in the other chapters, such as the influence of the number of body-surface electrodes on inverse reconstruction accuracy. In all, the research questions formulated in the previous section are discussed, and to some extent answered, and used to discuss the future of ECGI. 


\begin{abstract}
Electrical activity at the level of the heart muscle can be noninvasively reconstructed from bodysurface electrocardiograms and a patient-specific torso-heart geometry. This modality, coined electrocardiographic imaging, could fill the gap between the noninvasive (low-resolution) 12lead ECG and invasive (high-resolution) electrophysiology studies. Much progress has been made to establish electrocardiographic imaging, and clinical studies appear with increasing frequency. However, many assumptions and model choices are involved in its execution, and only limited validation has been performed. In this chapter, we will discuss the technical details, clinical applications and current limitations of commonly used methods in electrocardiographic imaging. It is important for clinicians to realize the influence of certain assumptions and model choices, for correct and careful interpretation of the results. This, in combination with more extensive validation, will allow for exploitation of the full potential of noninvasive electrocardiographic imaging as a powerful clinical tool to expedite diagnosis, guide therapy and improve risk stratification.
\end{abstract}

Adapted from: MJM Cluitmans, RLM Peeters, RL Westra, PGA Volders. Noninvasive reconstruction of cardiac electrical activity: update on current methods, applications and challenges. Neth Heart J, 2015, 23, 301-311 


\subsection{INTRODUCTION}

The 12-lead electrocardiogram (ECG) is a well-established, patient-friendly, quick, reproducible, and cheap tool to determine normal cardiac activation and repolarization, to diagnose cardiac arrhythmias, altered activation, ischemia, infarction, primary electrical abnormalities of the heart, structural disease, metabolic disorders, electrolyte imbalance and other conditions. It reflects the attenuated and dispersed result of propagated electrical activity and recovery in the heart on the body surface. However, it lacks the capacity to directly assess electrical activity at the level of the myocardium at high resolution. A modality that noninvasively images electrical activation and recovery at the heart surface could fill the gap between the noninvasive (low-resolution) 12-lead ECG and invasive (high-resolution) electrophysiology studies (EPs). Among other applications, this could facilitate the characterization and imaging of electrical gradients under physiological and pathological conditions, to localize the origins and circuits of ventricular tachycardias, to determine the substrate complexity underlying atrial fibrillation, and to assess the size and location of an infarct scar, which are important for risk stratification.

In this regard, a current challenge is the inverse problem of electrocardiography, which is the main topic of this chapter. The inverse problem of electrocardiography indicates our current limitations to determine the electrical activity at the level of the heart muscle by means of body-surface ECGs and a patient-specific torso-heart geometry. The relation between electrical heart activity and its projection on the body surface depends on the torso-heart geometry and thoracic conductivities. The anatomical reference for a patientspecific torso-heart geometry can be provided by imaging modalities such as CT or MRI. The cardiac electrical activity can then be reconstructed in terms of local electrograms, depolarization and repolarization isochrones, activation sequences, and other relevant electrophysiological signals.

Durrer et al. [1] were the first to thoroughly investigate the electrical activity of the intact human heart at the organ level. In their studies, they used explanted human hearts from patients who had died in the absence of heart disease. Years later, attempts to achieve this noninvasively were published in analytical and computer models [14], [22]-[30], experimental dogs [31], torso-tank experiments with isolated canine hearts [32], [33], and humans [34], [35].

During the last decades, much progress has been made in solving the inverse problem of electrocardiography [36]-[40], and applications in humans appear with increasing frequency. [34], [35], [41] Notably, the group of Yoram Rudy at Washington University in St. Louis, USA, has published a diverse series of clinical studies in the last years, e.g. [42]-[48]. In previous investigations in the Netherlands, noninvasive re- 


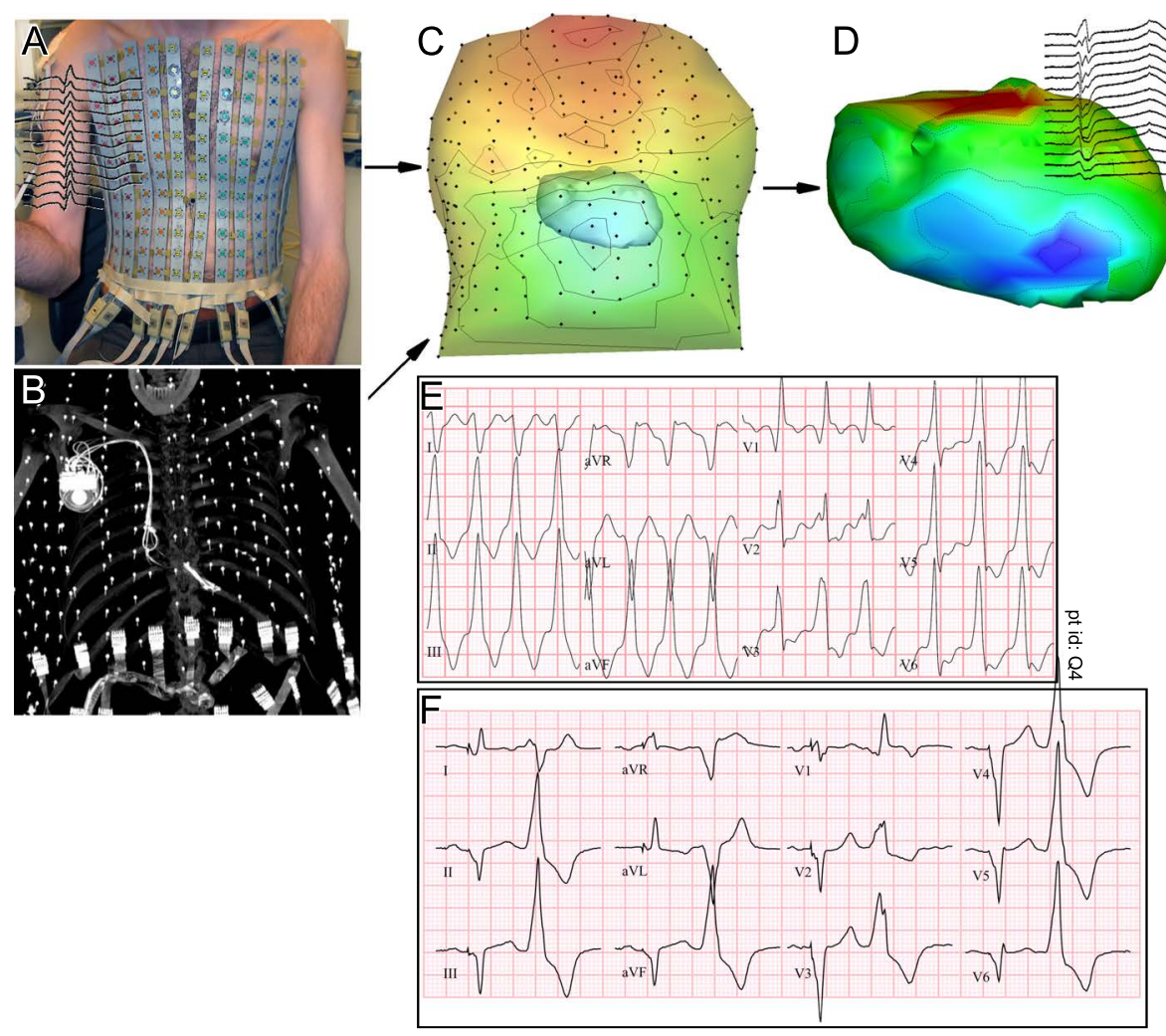

Figure 2.1: Inverse reconstruction of epicardial potentials in the patient from the text. Body-surface potentials are measured with 256 electrodes on the patient's torso $(A)$ and the geometrical and conductivity relationship between heart and body surface is, in this case, determined by computed tomography (B). The patient-specific inverse model (C) is then used to reconstruct epicardial potentials (D). Panel E shows the patient's VT, which shared morphology with his frequent VES beats (panel F: first beat paced, second beat VES). (Person in panel $A$ is not the patient.)

construction of electrical heart activity was studied as well [15], [27], [49]-[52], and clinical collaborations are pursued in the upcoming years [15], [53]. These methods carry different names, such as noninvasive electrocardiographic imaging (ECGI or ECGimaging, coined by the team of Yoram Rudy) [34], myocardial activation imaging [41], electrocardiographic mapping [54], inverse solution mapping [35], noninvasive imaging of cardiac electrophysiology (NICE) [55], three-dimensional cardiac activation imaging (3-DCAI) [56], noninvasive epi- and endocardial electrophysiology system (NEEES) [57], or similar terminology. We will refer to this full range of techniques as 'electrocardiographic imaging'. 
In the present chapter, we will discuss the different techniques available to noninvasively reconstruct electrical heart activity based on body-surface ECG recordings and anatomical imaging. A patient case will illustrate the application of electrocardiographic imaging in our centre in Maastricht, see Figure 2.1. This patient, a 63-year old male, was known with third-degree AV block, for which he received a pacemaker. After developing cardiomyopathy his device was upgraded to a biventricular (BiV) pacemaker and implantable cardioverter-defibrillator (ICD). His heart failure worsened and he developed a high count (20\%) of ventricular extrasystolic (VES) beats. No genetic or ischemic causes could be found and treatment with drugs proved insufficient. Ultrasound repeatedly confirmed a low left ventricular ejection fraction of $20 \%$ and moderate mitral regurgitation. When readmitted with recurrent monomorphic sustained ventricular tachycardia (VT, morphology similar to his VES beats, see Figure 2.1), an origin at the aortomitral continuity was suspected (based on the 12-lead ECG), but during electrophysiology (EP) study, an epicardial origin close to the distal coronary sinus seemed more likely. The patient underwent electrocardiographic imaging before the EP study. His case will demonstrate how noninvasively reconstructed epicardial electrograms can help localize the origin of ventricular tachycardia, and how reconstructed activation isochrones are superior to QRS duration and morphology on the regular 12-lead ECG for determining the electrical synchrony in cardiac resynchronization therapy (CRT). In this paper, we will also point out current shortcomings and basic questions that need to be addressed before noninvasive electrocardiographic imaging can be fully accepted in the clinic. Understanding the methods and pitfalls of electrocardiographic imaging is essential for the correct interpretation of its results.

\subsection{Clinical applications}

Applications of noninvasive electrocardiographic imaging are summarized in Table 2.1. One of these is the ability to detect the origin of VT [45], [58]-[61]. Although catheter ablation of sustained monomorphic VTs has been very successful in the last years, recurrence of VTs is still an issue, requiring repeated ablation procedures. There is not only a substantial recurrence rate in the large group of myocardial-infarction related VTs, but especially also in (inherited) cardiomyopathies [74]. Noninvasive electrocardiographic imaging may expedite EP studies and improve outcome, particularly in cases where the myocardium is very sensitive to catheter manipulation, or (such as the case presented in this paper) when only sporadic VES beats are present during the ablation procedure. These issues make intracardiac point-by-point mapping a strenuous task, whereas elec- 
Chapter 2. ECGI: a review on current methods, applications and challenges

\begin{tabular}{|c|c|c|}
\hline Application & Method requirements & Ref \\
\hline VT \& AT substrate & $\begin{array}{l}\text { (Sub-)centimeter resolution for relatively large } \\
\text { potentials; Endocardial vs. epicardial origin }\end{array}$ & {$[45],[58]-[64]$} \\
\hline AF substrate & $\begin{array}{l}\text { Millimeter resolution for relatively small poten- } \\
\text { tials }\end{array}$ & {$[44]$} \\
\hline MI substrate & $\begin{array}{l}\text { Detection of low-amplitude and fractionated } \\
\text { electrograms }\end{array}$ & {$[46],[65]$} \\
\hline CRT optimization & $\begin{array}{l}\text { Super-centimeter resolution for large potentials } \\
\text { (electrical synchrony) }\end{array}$ & {$[54],[55],[66],[67]$} \\
\hline Accessory pathways & Sub-centimeter resolution & {$[68]-[70]$} \\
\hline $\begin{array}{l}\text { Repolarization abnor- } \\
\text { malities }\end{array}$ & $\begin{array}{l}\text { Centimeter resolution for relatively small poten- } \\
\text { tials }\end{array}$ & {$[43],[47],[71]-[73]$} \\
\hline $\begin{array}{l}\text { Risk stratification (ar- } \\
\text { rhythmogenesis) }\end{array}$ & $\begin{array}{l}\text { Detection of conduction slowing and repolariza- } \\
\text { tion abnormalities }\end{array}$ & {$[46],[65]$} \\
\hline
\end{tabular}

Table 2.1: Applications and method requirements for noninvasive reconstruction of electrical heart activity. VT: ventricular tachycardia. AT: atrial tachycardia. AF: atrial fibrillation. MI: myocardial infarction. CRT: cardiac resynchronization therapy.

trocardiographic imaging can be performed noninvasively for several hours and requires only one extrasystolic beat to reconstruct its origin. Localizing the origin of VT noninvasively can also reduce radiation burden and procedural complications, and improve ablation success rate. For similar reasons, the management of atrial tachycardia may be facilitated by electrocardiographic imaging as well [62]-[64]. Furthermore, the suggested ability of electrocardiographic imaging to differentiate between an epicardial versus endocardial VT origin [59] would allow to select the appropriate ablation setup beforehand.

Electrocardiographic imaging may also help to reduce the currently large group of CRT non-responders, as it has been shown that noninvasive reconstruction of local activation timing predicts clinical CRT response better than QRS duration or the presence of left bundle branch block (LBBB) [54], [55], [66], [67]. Despite the low voltage amplitudes, atrial fibrillation (AF) has also been characterized by noninvasive reconstruction, assessing AF activation patterns and complexity [44], and electrocardiographic imaging significantly reduces invasive procedural time during AF ablation [75].

Another important public-health problem is sudden cardiac death (SCD). Individual risk stratification for SCD is notably difficult [ 76 ]. Electrocardiographic imaging may prove useful for the risk prediction of SCD by its ability to image and quantify conduction and repolarization abnormalities, which are an important substrate for arrhythmias [43], [47], [71]-[73], [77], [78]. More specifically, the electro-anatomical characterization 

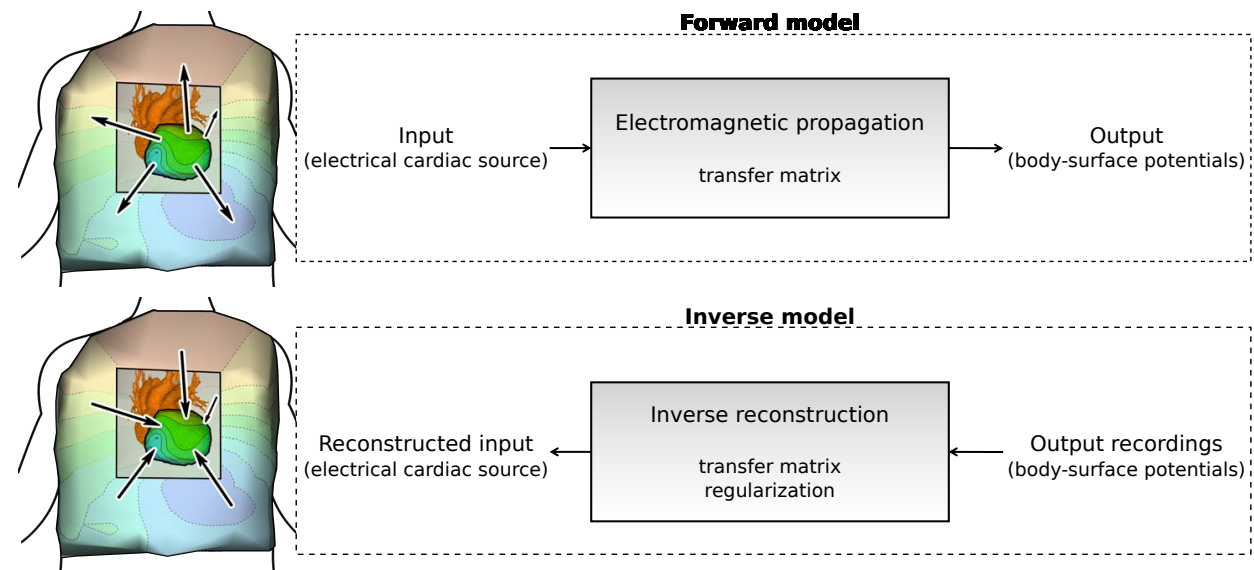

Inverse model

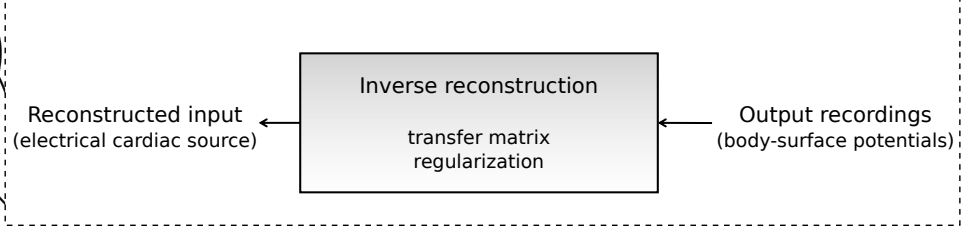

Figure 2.2: Schematic representation of forward/inverse models. A forward model describes the propagation of electromagnetic activity from the heart to the body surface; an inverse model reverses that relation, allowing for non-invasive reconstruction of electrical heart activity from measured body-surface potentials.

of scar tissue after myocardial infarction, myocarditis or cardiomyopathies by assessing local low-amplitude and fractionated electrograms, could contribute to risk stratification for VT and SCD [46], [65], [79].

Although all these examples illustrate the potential value of inverse electrocardiography, no specific application has been developed to such an extent yet that is has been included in a clinical guideline.

\subsection{INVERSE RECONSTRUCTION SETUP}

The setup necessary for inverse reconstruction consists of specialized hardware and mathematical algorithms. So-called 'forward models' describe the propagation of electromagnetic activity from heart to body surface, see Figure 2.2. An inverse model is based on a forward model with known 'output' and unknown 'source'; it essentially reverses the natural electromagnetic relationship between heart and body surface. Forward models consist of three parts: the cardiac source (representing the electrical activity of the heart), the model output (the body-surface potentials) and the electromagnetic source-output relation (capturing patient-specific propagation by an anatomical and conductivity reference). Reversing this electromagnetic relationship requires a fourth element, namely regularization methods, to deal with the reconstruction's sensitivity to noise. We will dis- 
Chapter 2. ECGI: a review on current methods, applications and challenges

\begin{tabular}{ll}
\hline Topic & Level of investigation \\
\hline Volume conductor & $\begin{array}{l}\text { (Piecewise) homogeneous: Investigated in model studies but } \\
\text { clinical implications unclear }\end{array}$ \\
Breathing and cardiac motion & $\begin{array}{l}\text { Assumed static: Not investigated } \\
\text { Torso volume }\end{array}$ \\
\hline
\end{tabular}

Table 2.2: General model assumptions and their level of investigation

cuss these four elements in the next sections. Some technique characteristics are summarized in Tables 2.2 and 2.3 .

\subsubsection{BODY-SURFACE POTENTIAL MEASUREMENTS}

To be able to reconstruct electrical activity of the heart, measurements of the projected potentials on the body surface should be taken at sufficient positions at anterior, posterior and lateral sides of the patient's torso. These represent the 'natural output' of the electromagnetic (forward) propagation of cardiac electrical signals to the body surface. However, the minimum number of body-surface electrodes and their optimal positioning remains a subject of discussion, but is usually taken to be far more than the nine electrodes of the 12-lead ECG. Currently used setups include 64 [41], 120 [35] or 256 [45] electrodes. Simulation studies indicate that when a limited number of electrodes is used, their positioning is of significant influence on the quality of the inverse reconstructions [80]. Recently, the use of only the standard 12-lead ECG was shown to be worthwhile in some cases, but using this small number of body-surface electrodes has not been validated thoroughly [53]. We typically use 256 electrodes for body-surface potential measurements (Figure 2.1A) with specialized hardware (BioSemi, Amsterdam, the Netherlands).

\subsubsection{CARDIAC SOURCE REPRESENTATION}

After measuring the body-surface potentials, a suitable model for the cardiac source must be chosen. Currently, two distinct representations for electrical heart activity are used most often: the potential-based formulation and the wave-front formulation. The potentialbased formulation is built on the assumption that there is a direct and unique relation between the potentials at the epicardial surface and the potentials at the body surface, described by the conductivity properties of the torso as a passive electrical conductor [14], [34], [35], [54]. In this approach, local electrograms at the epicardium are reconstructed, from which additional information can be obtained, such as activation and 


\begin{tabular}{|c|c|c|}
\hline & Potential-based formulation & Wave-front formulation \\
\hline \multicolumn{3}{|c|}{ Source representation } \\
\hline Source model & Epicardial potentials & Current dipole layers \\
\hline Endocardial/epicardial & Only epicardial representation & $\begin{array}{l}\text { Endo- \& epicardial represen- } \\
\text { tation }\end{array}$ \\
\hline \multicolumn{3}{|c|}{ Output measurements (body-surface potentials) } \\
\hline $\begin{array}{l}\text { Number and positioning of } \\
\text { body-surface electrodes }\end{array}$ & $\begin{array}{l}\text { Not established, 100+ elec- } \\
\text { trodes used }\end{array}$ & $\begin{array}{l}\text { Not established, } 64+\text { elec- } \\
\text { trodes used }\end{array}$ \\
\hline \multicolumn{3}{|c|}{ Regularization \& reconstruction } \\
\hline $\begin{array}{l}\text { Sensitivity to ill-posedness } \\
\text { (noise) }\end{array}$ & High & Intermediate \\
\hline Local electrograms & Yes, direct & Yes, indirect \\
\hline Epicardial information & Yes & Yes \\
\hline Endocardial information & Maybe, under debate & Yes \\
\hline Activation times & Yes, indirect & Yes, direct \\
\hline Repolarization times & Yes, indirect & Yes, direct \\
\hline Fractionated potentials & Yes & No \\
\hline Low amplitude potentials & Yes & No \\
\hline Conduction slowing & Yes & Yes \\
\hline \multicolumn{3}{|c|}{ Validation } \\
\hline In vitro & Torso tank, extensive & None \\
\hline Animal & None & $\begin{array}{l}\text { Limited (only asynchronous, } \\
\text { endocardial validation) }\end{array}$ \\
\hline Human & $\begin{array}{l}\text { Limited (asynchronous, open } \\
\text { thorax, or only endocardial) }\end{array}$ & $\begin{array}{l}\text { Limited (asynchronous, only } \\
\text { endocardial) }\end{array}$ \\
\hline \multicolumn{3}{|c|}{ Reconstruction quality } \\
\hline Detection of origin & Centimeter accuracy & Centimeter accuracy \\
\hline Spatial resolution & Not investigated & Not investigated \\
\hline Temporal resolution & Not investigated & Not investigated \\
\hline \multicolumn{3}{|c|}{ Clinical applications in literature } \\
\hline VT substrate & Yes & Yes \\
\hline AT substrate & Yes & No \\
\hline CRT optimization & Yes & Yes \\
\hline Accessory pathways & Yes & Yes \\
\hline AF characterization & Limited & No \\
\hline Myocardial infarction & Limited & No \\
\hline Repolarization abnormalities & Limited & No \\
\hline
\end{tabular}

Table 2.3: Characteristics of commonly used methods for noninvasive reconstruction of electrical heart activity. Details in text. VT: ventricular tachycardia; AT: atrial tachycardia; CRT: cardiac resynchronisation therapy; AF: atrial fibrillation. 
repolarization times, fractionated potentials, low-amplitude potentials etc. Some approaches aim at reconstructing endocardial potentials at the same time, although there is still discussion about the feasibility of this approach [81], [82].

The wave-front formulation utilizes different assumptions to model activation and repolarization wave-fronts, by defining the wave-front (or more recently the complete endocardium and epicardium) as layer of current dipoles [30], [41], [55], [56]. This approach is more restricted than the potential-based method, yielding only local activation and repolarization times. On the other hand, the more sparse representation of the wave-front approach makes it less sensitive to noise. Furthermore, this method enables both endocardial and epicardial information to be obtained. In the approach applied by the group of Van Oosterom in the Netherlands, estimates for the cardiac source are used to solve the forward model to compute the corresponding body-surface potentials, which are then compared with the measured body-surface potentials [15]. Via an iterative approach an optimal estimate of the activation sequence is computed to best match the measured body-surface potentials [83]. Recently, Van Oosterom investigated the relation between the wave-front and potential-based methods and showed in a simulated case that also local electrograms can be computed from the wave-front formulation [40].

Other methods formulate the cardiac source fundamentally differently. For example, attempts were made to directly reconstruct areas of myocardial infarction [84]. Another method is to reconstruct transmembrane potentials, a 'bidomain' approach that connects computational models of intracellular and extracellular domains to body-surface potential measurements $[85]-\left[8_{7}\right]$. These approaches have not yet gained as much attention as the potential-based or wave-front formulation.

\subsubsection{SOURCE-OUTPUT RELATION: ANATOMICAL AND CONDUCTIVITY REFERENCE}

The next step in inverse reconstruction of electrical heart activity is determining the electromagnetic relation between source and output. This relation is captured by a patientspecific transfer matrix, based on conductivity and geometry of the patient's torso. This is essential for creating accurate, patient-specific reconstructions that can be linked to anatomical location and abnormalities. A CT or MRI scan is used to obtain the anatomical reference (Figure 2.1B), from which a geometry is created that consists of the heart surface, the location of the body-surface electrodes, and the torso-volume conductor properties (Figure 2.1 C). The heart surface can either be defined purely by the epicardium (in the potential-based approach), or by both endocardium and epicardium (in the wave-front approach). 


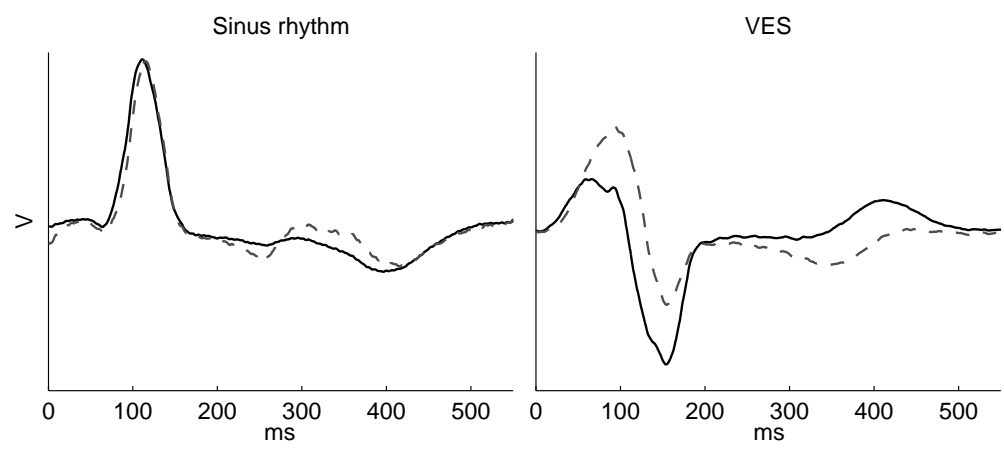

Figure 2.3: Reconstructed epicardial electrograms on one location of the left ventricle (indicated with an asterisk in Figure 2.6) of a 63-year old patient with native LBBB during sinus rhythm and frequent ventricular extrasystoles (VES). In the reconstruction process, the same body-surface potentials (either sinus rhythm with LBBB or VES), but a different torso-heart geometry were used: one with a diastolic cardiac geometry (resulting in the electrograms with a solid line), and one with a systolic cardiac geometry (dashed line). Using either a systolic or diastolic cardiac geometry results in significantly different electrograms on the same epicardial location, notably in the repolarization phase. This indicates that cardiac contractile movement should be taken into account when reconstructing epicardial potentials. Voltage scales identical for both graphs.

It is assumed that the torso volume can be divided into subregions with constant conductivity. In its simplest form, the human torso can be represented as completely homogeneous and containing only the heart. More realistic representations use a piecewise homogeneous torso, including other tissues such as the lungs, fatty tissue, bones and skeletal muscles, each with their specific conductivity. Some simulation studies suggest that the inclusion of torso inhomogeneities is necessary [40], [88], [89], whereas others have revealed that the reconstructed epicardial potentials were only slightly less accurate when omitting inhomogeneities [90]. In-vivo studies are needed to assess the clinical relevance of including torso inhomogeneities in humans and whether it is relevant to create a more complicated geometry.

Another neglected element in the computation of the transfer matrix is the false assumption that both torso and heart are non-moving. The static torso-heart geometry is usually based on a CT scan taken during cardiac diastole and breath-hold, to capture both heart and torso in a quiescent state. However, during the recording of body-surface potentials that are used for inverse reconstruction, both torso and heart are changing in geometry (and conductivity). Especially the reconstruction of repolarization would suffer from the assumption of a static (diastolic) geometry, as we demonstrate for the patient in Figure 2.3 where we used both a diastolic and a systolic geometry for recon- 
structing the same body-surface potentials on the epicardium. Clearly, the influence of using a static geometry should be investigated further in clinical studies.

Furthermore, the need for a CT or MRI scan hinders quick application of electrocardiographic imaging, complicates logistics in daily clinical practice, and induces radiation burden to the patient when $\mathrm{CT}$ is used.

\subsubsection{RECONSTRUCTION AND REGULARIZATION METHODS}

After 1) measuring the body-surface potentials, 2) choosing a suitable cardiac source representation, and 3 ) defining the electromagnetic relation between those two elements with a patient-specific transfer matrix, noninvasive reconstructions of cardiac electrical activity can be performed with an inverse model. However, the inverse problem is inherently ill-posed. This means that the solution of the problem is extremely sensitive to small perturbations in the measured body-surface potentials, such as noise. Ill-posedness is the mathematical consequence of the attenuating and dispersing effect of the electromagnetic propagation from heart to body surface [91]. Most implementations of the inverse problem therefore suffer from numerical instability. So-called 'regularization methods' are needed to deal with this uncertainty and sensitivity in inverse models.

Regularization incorporates additional knowledge in the inverse problem by applying constraints to the solutions, which will yield more realistic results, see Figure 2.4. These additional constraints are based on physical or mathematical properties that apply to the forward/inverse models but that are not yet incorporated in the transfer matrix. When regularization is applied, the weight of the constraints (the regularization parameter) has to be determined to find a balance between solutions purely based on the bodysurface potentials (possibly severely distorted by ill-posedness) and solutions that are constrained too strictly (possibly too much bias). Methods exist that aim at finding an optimal balance [92]. The important influence of the regularization parameter is shown in Figure 2.5 for the same patient as described previously.

The most commonly used regularization method is Tikhonov regularization, which is based on the idea that epicardial potentials should be reasonably small (zeroth order Tikhonov) or smoothly changing over the heart surface (first and second order Tikhonov) [93]. Other regularization methods include truncated Singular Value Decomposition (tSVD) [94], Greensite SVD [95], and the Generalized Minimal Residual (GMRes) method [96]. Each method has its specific advantages and disadvantages, but all have difficulties with the inherent disadvantageous properties of the ill-posed inverse problem. Moreover, reconstruction quality can heavily depend on parameter settings. 


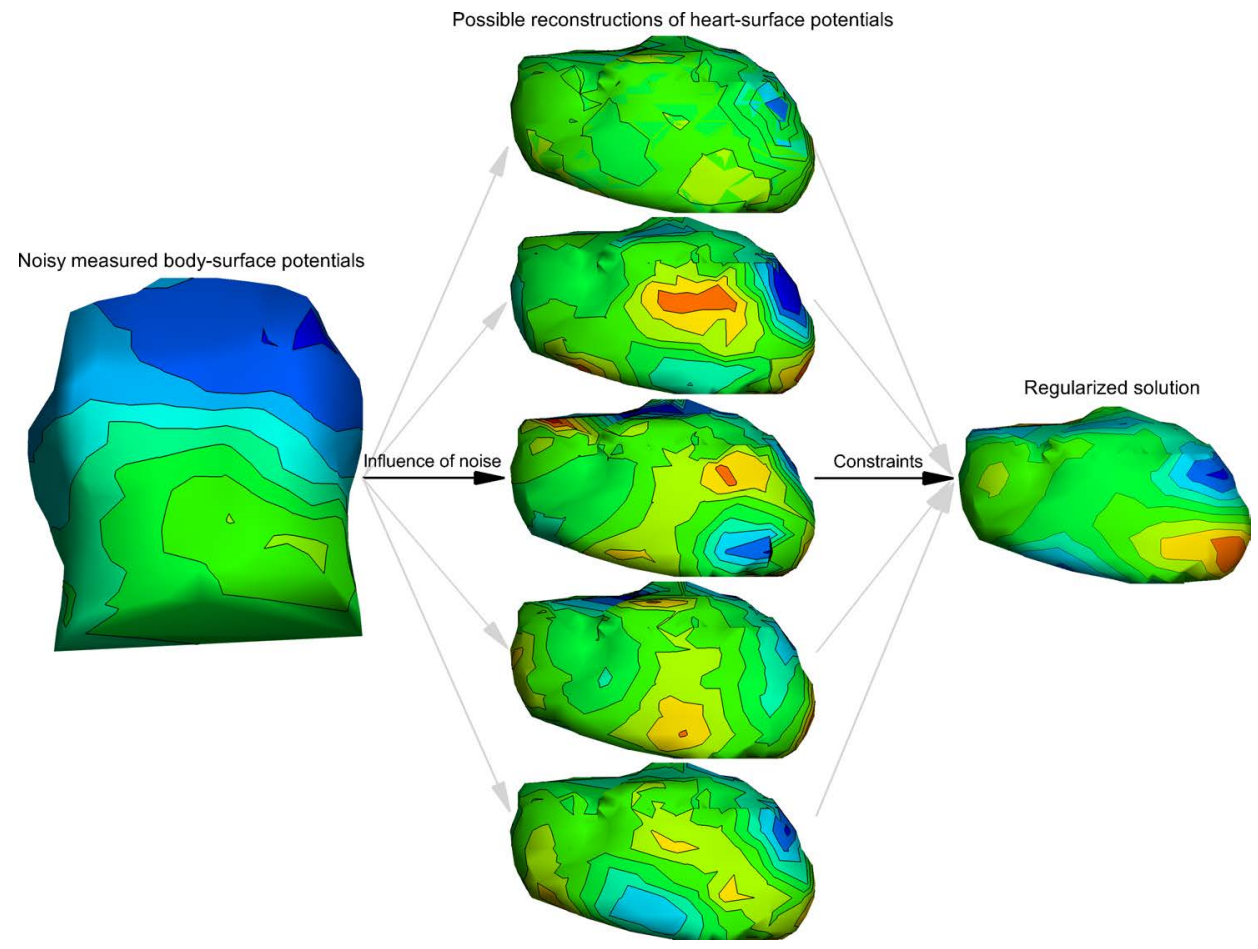

Figure 2.4: III-posedness: small perturbations in the input data (such as noice in recorded body-surface potentials) result in completely different reconstructions. By applying contraints, a regularized solution is obtained.

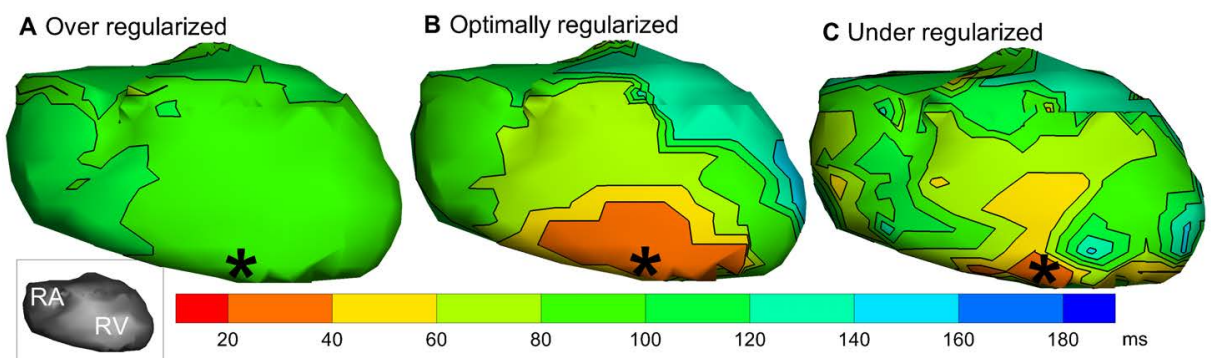

Figure 2.5: Noninvasively reconstructed isochrones of a paced beat, on the right ventricle (RV) of the same patient as in Figure 2.3, for different values of the regularization parameter. Panel A shows an overregularized setting, applying the constraints too heavily, resulting in a reconstruction that does not contain any relevant information. An optimally regularized solution provides the most adequate reconstruction (panel B), showing early activation (orange colour) on the location of pacing (indicated with an asterisk). An underregularized setting results in a reconstruction that is dominated by the influence of noise (panel $C$ ). 
Recently, a first attempt was made to not only use physical and mathematical properties to regularize the solution, but to incorporate electrophysiological information as well to improve the quality of the reconstruction [97]. For an in-depth review of regularization methods, we refer to Milanic et al [98].

It is important for clinicians to realize that constraints are introduced purposefully to reach realistic solutions, but that this bias might create artefacts or prevent the reconstruction of electrical activity that is not accounted for by the constraints that were added.

\subsection{RECONSTRUCTION QUALITY AND CLINICAL VALIDATION}

In the selected case from our patient studies, we obtained body-surface potentials with 256 electrodes and chose a potential-based representation of the cardiac source. The most important reason for doing so is to be able to reconstruct local electrograms and not only activation/repolarization timing. Moreover, it has been validated more thoroughly in clinical settings. The patient-specific geometry, based on a CT scan during diastole, was chosen to be homogeneous. As a regularization method, we applied Tikhonov zeroth order regularization. Reconstructed electrograms are shown in Figure 2.6, for three different beats in this patient. The insets in panel A and B show pseudo-unipolar electrograms recorded at the pacemaker lead tips in the right and left ventricle that correspond to the location of the shown reconstructed electrograms. Correlation coefficients between measured and reconstructed electrograms range from 0.79 to 0.85 . Although the correlation coefficients are reasonably high, some important features are clearly not reconstructed correctly. This might be due to a combined effect of the pseudo-unipolar character of the pacemaker recordings, cardiac changes in the months between recording of body-surface potentials and pacemaker electrograms, the location mismatch in case of the right ventricle (endocardial recording vs epicardial reconstruction), and shortcomings in the inverse algorithm.

Activation isochrones can be created by determining the activation time at each epicardial location, as shown in the last two columns of Figure 2.6. Activation of a sinus beat (Panel A) starts at the right ventricle (red) and spreads to the left ventricle (blue), as was expected based on the LBBB morphology on the 12-lead ECG. Panel B shows activation isochrones for a paced beat ( $\mathrm{BiV}$ paced). On the right ventricle, the reconstructed location with earliest activation (indicated with symbol \#) is $23 \mathrm{~mm}$ from the known pacing location (indicated with symbol ${ }^{*}$ ) that was determined from the anatomical reference. For the left ventricle, the reconstructed and known locations of first activation are $28 \mathrm{~mm}$ apart. In agreement with pacemaker settings, the right ventricle was paced and activated 

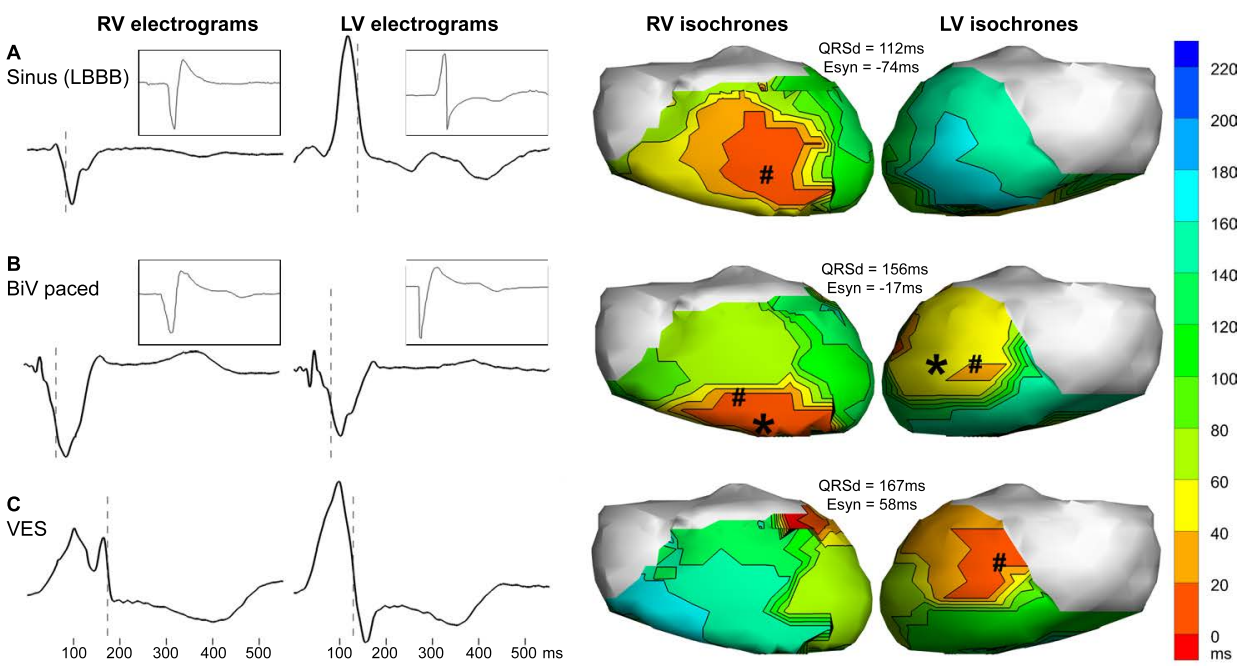

Figure 2.6: Reconstructed epicardial electrograms (first two columns, voltage scales identical over all graphs) and reconstructed activation isochrones (last two columns) for three different beats (row A: sinus beat with LBBB pattern; B: biventricularly paced beat; C: extrasystolic beat, see Figure 2.1F for 12-lead ECG of the VES). The reconstructed electrograms correspond to the epicardial location closest to the right and left pacing lead tips from the patient's pacemaker. The insets show recorded electrograms from those leads on the same location for comparison, i.e. an endocardial recording for the right ventricle, and an epicardial recording for the left ventricle. The dashed line indicates the activation time (maximum $-\mathrm{dV} / \mathrm{dt}$ ). The ventricular activation isochrones, depicted in the last two columns, reflect the time of maximum $-\mathrm{dV} / \mathrm{dt}$ per epicardial location. Locations of reconstructed earliest activation are indicated with symbol \#. QRS duration (QRSd) is based on the 12-lead ECG. Electrical synchrony (Esyn) is the difference between the mean activation times at the right ventricle (RV) and the mean activation times at the left ventricle (LV); a value close to zero usually results in more efficient contraction. For the paced beat (panel B), the pacing locations are indicated with symbol *.

before the left ventricle. The activation isochrones of a VES beat (Panel C) suggest an origin of extrasystolic activity on the superior part of the left ventricle, although spatial resolution was too low to give a very precise location. From the EP study, an epicardial location at the mid-coronary sinus was suspected to be the origin, consistent with these reconstructions.

Figure 2.6 also demonstrates the advantage of inverse electrocardiography in CRT optimization, by showing that electrical synchrony, i.e., measuring the time difference of activation of the complete left versus the complete right ventricle based on inversely reconstructed electrograms, gives better insight in ventricular (dys)synchrony than the often used 12-lead QRS duration.

Although reconstructed electrograms and activation times are consistent with ex- 
pectations based on 12-lead ECGs, pacemaker recordings and the EP study, the level of accuracy and detail is suboptimal, with correlation coefficients around 0.80 and a reconstructed pacing-origin mismatch of $23-28 \mathrm{~mm}$. Other research groups have performed more extensive validation studies, claiming $10 \mathrm{~mm}$ accuracy and cross correlations of 0.70. [78] Other studies have found a significant decrease of accuracy in diseased hearts, with localization accuracy going from $13 \pm 9 \mathrm{~mm}$ (mean $\pm \mathrm{SD}$ ) in healthy hearts up to $28 \pm 27 \mathrm{~mm}$ in infarcted hearts and even $43 \pm 11 \mathrm{~mm}$ near scarred tissue. [35] However, no thorough in-vivo validation has been performed with potential recordings simultaneously at extensive locations at the heart and body surfaces in intact organisms.

\subsection{Limitations, ADDED VALUE AND FUtURE POTENTIAL}

The potential applications of noninvasive reconstruction of electrical heart activity are promising. Localization of extrasystolic activity in a noninvasive, per-beat and precise manner combined with an anatomical reference can expedite diagnosis, guide therapy and reduce procedural radiation burden. Electrical (dys)synchrony assessment allows patient-specific CRT and might help in improving therapy for current non-responders. Future applications could also include noninvasive risk stratification for conduction abnormalities (conduction slowing, electrical chaos in AF, low-voltage amplitudes), direct relation of structural abnormalities to electrical abnormalities (fibrosis, fibrofatty replacement) and easier visual interpretation of abnormalities that are already diagnosed on the powerful 12-lead ECG. Commercial, easy-to-use setups such as those developed by CardioInsight (Cleveland, OH, USA) will be essential to fully exploit the potential of these applications in daily clinical routine.

Whereas noninvasive reconstruction of electrical heart activity currently has a clear research potential, its added value in clinical practice remains to be established. It is still unclear when, from a patient and socioeconomic perspective, it is worth the extra effort of extensive mapping of body-surface potentials, performing a CT/MRI scan for anatomical reference, and carefully avoiding the regularization and reconstruction pitfalls. More importantly, cardiologists should be aware of the existence and influence of those pitfalls and the assumptions underlying the reconstruction algorithms. Debate remains on topics such as the influence of inhomogeneities in the torso (notably the lungs) on the reconstructions, the optimal regularization methods and their parameters, the maximum achievable resolution of reconstruction, the number and positioning of bodysurface electrodes and the assumption of a static geometry. We feel that more extensive validation should be performed, besides additional clinical studies investigating inverse electrocardiographic imaging for various purposes. Extensive validation by in-vivo 
studies, and careful interpretation of clinical results with knowledge about the underlying methods and assumptions, will allow full exploitation of the potential of noninvasive electrocardiographic imaging, improving diagnosis, therapy and risk stratification.

\section{ACKNOWLedgements}

The authors would like to thank Dr. Bas L.J.H. Kietselaer, cardiologist, and Dr. Marco Das, radiologist, Maastricht University Medical Centre, for their contribution in obtaining the CT scans in the patient studies, and Dr. Joël M.H. Karel and Dr. Pietro Bonizzi, Department of Knowledge Engineering, Maastricht University, for their valuable technical discussions.

\section{CONFLICTS OF INTEREST}

Conflict of interest: none declared 


\section{Abstract}

Background: Noninvasive imaging of electrical potentials at the epicardium, known as electrocardiographic imaging (ECGI), is increasingly applied in patients to assess normal and abnormal cardiac electrical activity.

Objectives: To evaluate the accuracy of noninvasive reconstructions of epicardial potentials, electrograms, activation and recovery isochrones and beat origins by simultaneously performing ECGI and invasive epicardial electrography in intact animals.

Methods: Body-surface potentials and epicardial potentials were recorded in normal anesthetized dogs. CT scanning provided a torso-heart geometry that was used to reconstruct epicardial potentials from body-surface potentials.

Results: Electrogram reconstructions attained a high accuracy compared with epicardial recordings (median correlation coefficient (CC): 0.71 ), but with considerable variation (interquartile range (IQR): $0.36-0.86$ ). This variation could be explained by a spatial mismatch (overall resolution was $<20 \mathrm{~mm}$ ) that was most apparent in regions with electrographic transition. Variability in electrogram quality was addressed by a spatiotemporal approach that incorporates the characteristics of neighboring electrograms as well, enabling more accurate derivation of activation times (Pearson's R: 0.82 ), recovery times (R: 0.73 ), and the origin of paced beats (median error $10 \mathrm{~mm}, \mathrm{IQR}$ 7-17 mm). Reconstruction of beats from repeated single-site pacing showed a stable localization of origin. Cardiac motion, currently ignored in ECGI, correlates negatively with reconstruction accuracy.

Conclusions: ECGI shows a high median accuracy, but variability in electrogram reconstruction can be sizable. Thus, at present, clinical interpretations of ECGI should not be based on single electrograms only. Incorporating local spatiotemporal characteristics enhances the reconstruction of epicardial activation and recovery patterns, and beat origin localization to $10 \mathrm{~mm}$ accuracy. Even more reliable interpretations are expected when the influences of cardiac motion are accounted for in ECGI.

Adapted from: MJM Cluitmans, P Bonizzi, JMH Karel, M Das, BLJH Kietselaer, MMJ de Jong, FW Prinzen, RLM Peeters, RL Westra, PGA Volders. In vivo validation of electrocardiographic imaging. In revision. 


\subsection{INTRODUCTION}

As discussed in Chapters 1 and 2, noninvasive electrocardiographic imaging (ECGI) reconstructs epicardial potentials, electrograms and activation/recovery isochrones from body-surface electrograms. [34] It is based on the relation between potentials at the heart surface and on the torso, dictated by the laws of electromagnetism. Inverting this relation enables to reconstruct epicardial potentials from electrocardiograms recorded at the body surface. This requires a thorough understanding of the heart-torso relation based on the patient-specific geometry and conductivity. Anatomical input is mostly provided by computed tomography (CT) or magnetic resonance imaging (MRI). Additionally, mathematical methods are necessary to overcome the uncertainties that emerge when assessing epicardial potentials from their attenuated and superimposed projections on the torso. Chapter 2 addressed these technical aspects and their clinical implications. [99]

ECGI has been used to noninvasively characterize normal activation and recovery in healthy human subjects. [42] A main focus has also been to detect the origin of ventricular or atrial tachyarrhythmias, thus expediting diagnostic catheter-based electrophysiology studies and potentially promoting the successful outcome of ablation. [45], [62] ECGI of atrial fibrillation (AF) has helped to reduce invasive procedural time during ablation. [75] Noninvasive mapping with ECGI provided detailed electrical activation patterns of the left $(\mathrm{LV})$ and right ventricle (RV), which could improve resynchronization therapy. [54] Likewise, ECGI can benefit risk stratification for sudden cardiac death by imaging of activation and recovery abnormalities, as important arrhythmogenic substrates. [71], [72]

Considering all these applications, it is remarkable that invasive validation studies of ECGI in intact organisms have been limited. Previous investigations were performed using analytical and computer models [22], and computer models with partially real data [71], or were based on torso-tank experiments with isolated canine hearts [33], experiments in dogs [31] and to some extent in humans [35], [44], [78]. Each of these studies had distinct advantages, such as the amount of control in the torso-tank experiments and the potential of clinical application in the human studies. However, none of these studies compared noninvasive reconstructions with simultaneous invasive electrograms in an intact, closed-chest organism. For the present study, we examined the reconstruction accuracy and capabilities of ECGI with high-precision simultaneous heart and torso recordings from uniquely-instrumented anesthetized dogs, see Figure 3.1. 

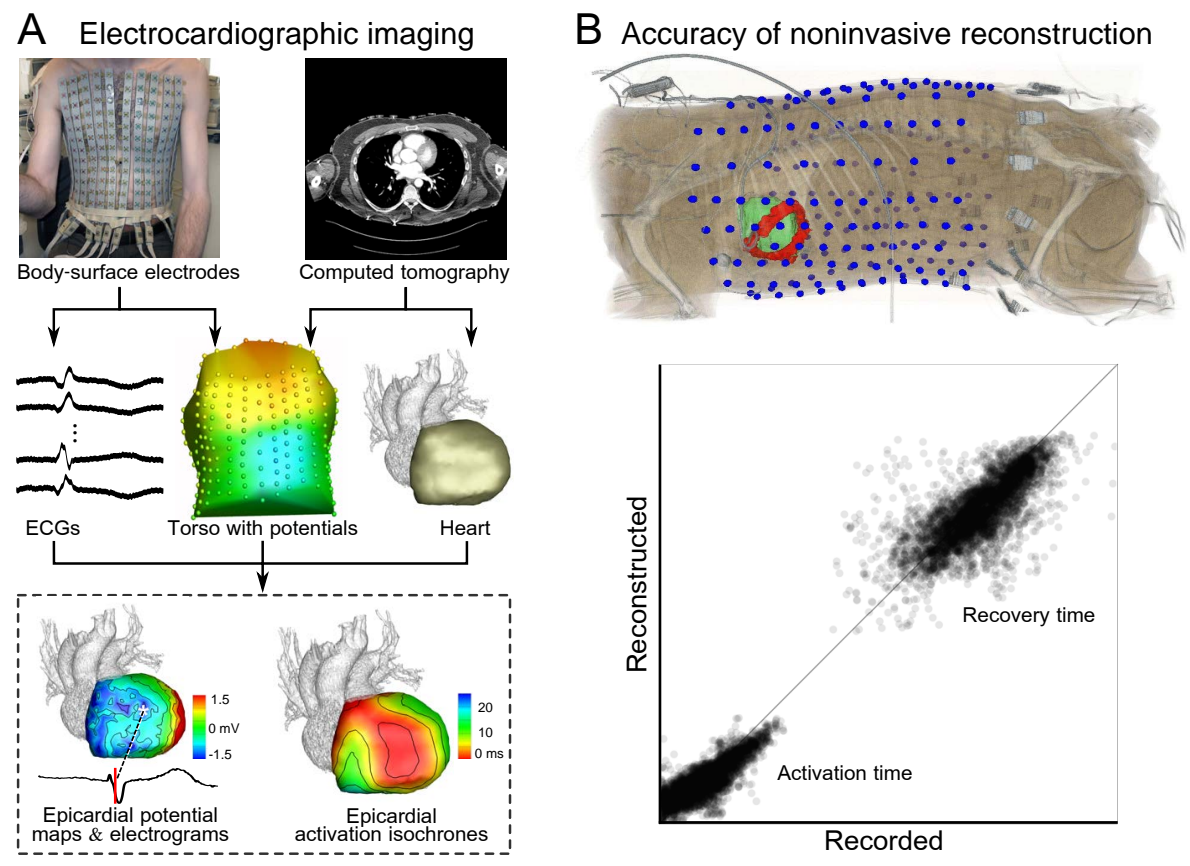

Figure 3.1: Electrocardiographic Imaging (ECGI) noninvasively reconstructs electrograms and activation and recovery isochrones on the epicardium, which were evaluated in canine experiments. Panel A: Noninvasive ECGI as it is generally applied in humans. Body-surface ECGs are combined with a torso-heart geometry obtained with $\mathrm{CT}$. By carefully reversing the physical laws of electromagnetism, epicardial potentials can be reconstructed. From these, epicardial electrograms and isochrones are deducted. Panel B: The experimental setup as applied in a normal anesthetized dog in the validation experiments of this Thesis, illustrating the body-surface electrodes (blue), the epicardial surface (green) and the epicardial electrodes (red). The graph shows that activation and recovery times are accurately reconstructed with ECGI.

\subsection{Methods}

Here, we discuss the main methods. For a detailed description, we refer to Section 3.6 of the Data Supplement at the end of this Chapter.

\subsubsection{ANIMAL EXPERIMENTS}

This investigation conformed to the Guide for the Care and Use of Laboratory Animals published by the United States National Institutes of Health (National Institutes of Health Publication 85-23, revised 1996). Animal handling was in accordance with the European Directive for the Protection of Vertebrate Animals Used for Experimental and 
Other Scientific Purposes (86/609/EU) and was approved by the institutional review committee for animal studies.

In four normal anesthetized dogs, two silicone bands with 99 electrodes were implanted around the basal and mid-basal ventricular epicardium after thoracotomy. Each band consisted of two rows of electrodes. Additional electrodes were placed at the LV apical epicardium, the $\mathrm{LV}$ endocardium, the RV apical endocardium, and the right atrial endocardium, providing a total of 103 electrodes. After chest closure, body-surface electrodes (184-216, depending on torso size) were attached to the chest. Unipolar potential recordings were obtained simultaneously by the epicardial and body-surface electrodes. Recordings were performed on the CT table to avoid a change in the geometry or disconnection of the electrodes by moving the animals. A helical ECG-gated CT scan was performed with intravenous iodine contrast medium and a diastolic reconstruction of the torso-heart geometry was performed.

\subsubsection{INVERSE RECONSTRUCTION}

Inverse reconstruction of epicardial potentials was identical to the human application illustrated in Figure 3.1A, and is based on the potential-based formulation of ECGI. This formulation assumes that there is a numerical relation between electrical potentials at the heart and body surface. [99] It is currently the most used formulation, and is also available in commercial setups.

A torso-heart geometry was digitized from the CT scan and contained the bodysurface electrodes and the ventricular epicardial surface (consisting of on average 1693 nodes, mean node-to-node distance $4 \mathrm{~mm}$ ). Additionally, the position of the $103 \mathrm{im}$ planted electrodes was digitized. In the resultant torso-heart geometry, we did not include torso inhomogeneities (such as lungs, bones or fat tissue).

Direct inverse reconstruction of epicardial potentials from the recorded bodysurface potentials is subject to uncertainty due to the ill-posed character of the inverse problem. Commonly used methods were applied to minimize this and to reconstruct epicardial potentials. [99] We refer to 'nodes' as the virtual points on the epicardial surface on which potentials are reconstructed, and to 'electrodes' as the physically implanted electrodes that record epicardial potentials.

\subsubsection{Post-PRoCesSing TO OBTAIN ELECTROGRAMS, ISOCHRONES AND ACTIVATION ORIGIN}

After reconstruction of epicardial potentials, electrograms were reconstructed per node by concatenating potentials over time. Activation times were determined per electro- 
gram with two different methods: the temporal-only and a spatiotemporal method. The temporal-only approach defines the moment of activation as the moment of steepest voltage downslope (maximum $-d V / d t$ ) during the QRS complex. The spatiotemporal approach, proposed by Erem et al [100], takes advantage of the spatial relationship between neighboring nodes and their potentials, and could be better suited to estimate the activation time in noisy or fractionated electrograms. This approach selects the moment that matches the change in temporal derivative with the change in spatial derivative. [100] Recovery times were defined as the moment of maximum $d V / d t$ during the electrographic $\mathrm{T}$ wave.

All reconstructions and analyses were performed automatically to prevent bias by the researchers and investigate the robustness and practicality of the technique.

\section{$3 \cdot 3$ Results}

In the 4 dogs, 140 beats were analyzed: 93 morphologically unique beats (where only one beat per pacing site was included), and 47 additional non-unique beats (repeated single-site pacing) at 4 different locations. In the 93 unique beats, on average 60 epicardial electrodes provided high-quality potential recordings, resulting in 5552 pairs of recorded and reconstructed electrograms. A large diversity of beats was analyzed: normally conducted sinus beats ( $\mathrm{n}=6$ beats), atrially paced beats with normal ventricular activation $(n=7)$, and beats paced from the $\mathrm{LV}$ endocardium $(\mathrm{n}=3), \mathrm{RV}$ endocardium $(n=2)$, ventricular epicardium $(n=71)$ or by biventricular epicardial pacing $(n=4)$. The additional 47 beats occurred upon repeated pacing at 4 different locations, to determine the stability of origin localization.

\subsubsection{ACCURACY OF RECONSTRUCTED ELECTROGRAMS}

Figure 3.2 shows representative examples of recorded and reconstructed electrograms for a sinus beat (panel A) and an LV epicardially paced beat (panel B). Median correlation coefficient was 0.71 for all 5552 pairs of recorded and reconstructed electrograms. There was a considerable (skewed) spread in correlation coefficients, resulting in an interquartile range (IQR, spanning the $25-75 \%$ range of data) of $0.36-0.86$. Of the electrograms with a correlation coefficient $<0.40,65 \%$ was found in a region with changing electrogram morphologies, as illustrated in panel C for electrode 88 of the paced beat (computational details in Section 3.6.5 in the Data Supplement at the end of this Chapter). The actual percentage is probably higher, because such transitions could only be detected in the direction of the electrode band, and were missed when occurring perpendicular to it. 
A
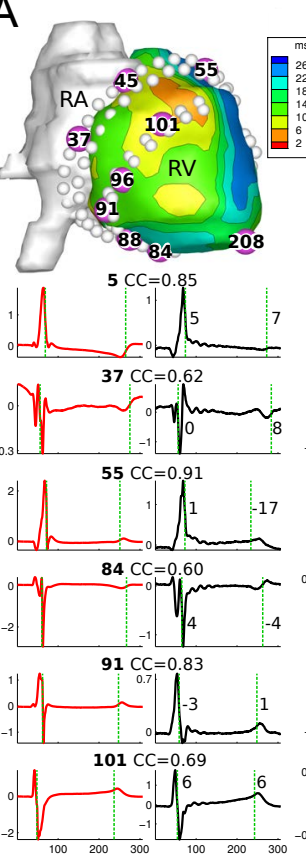
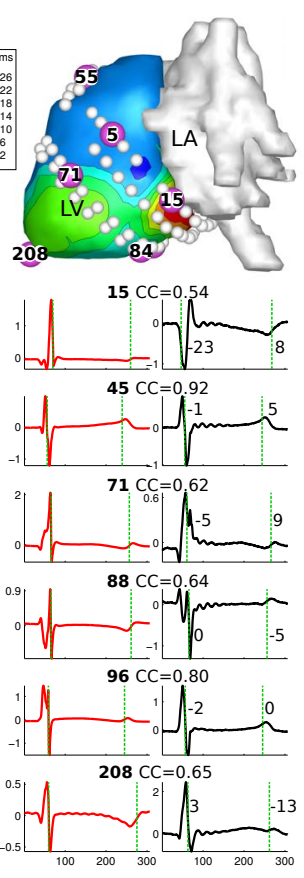

B
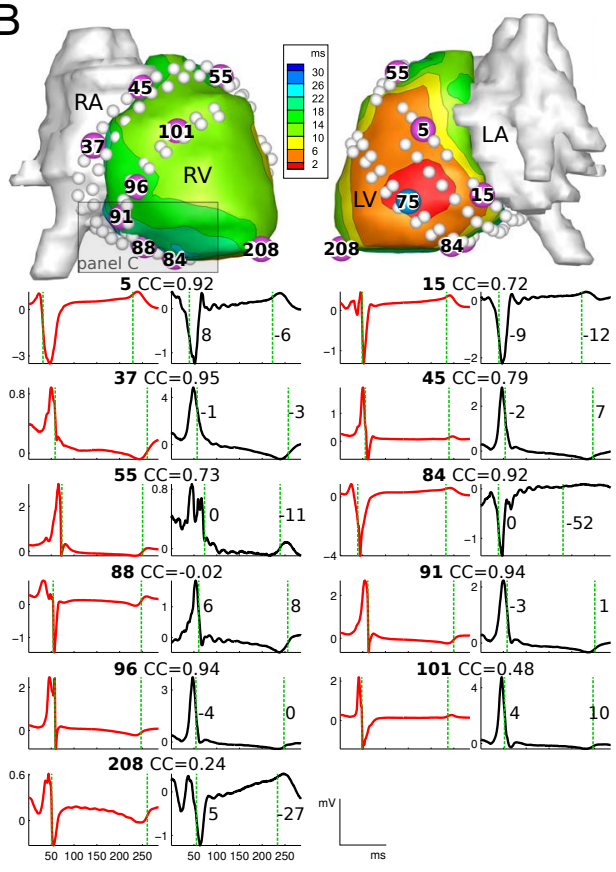

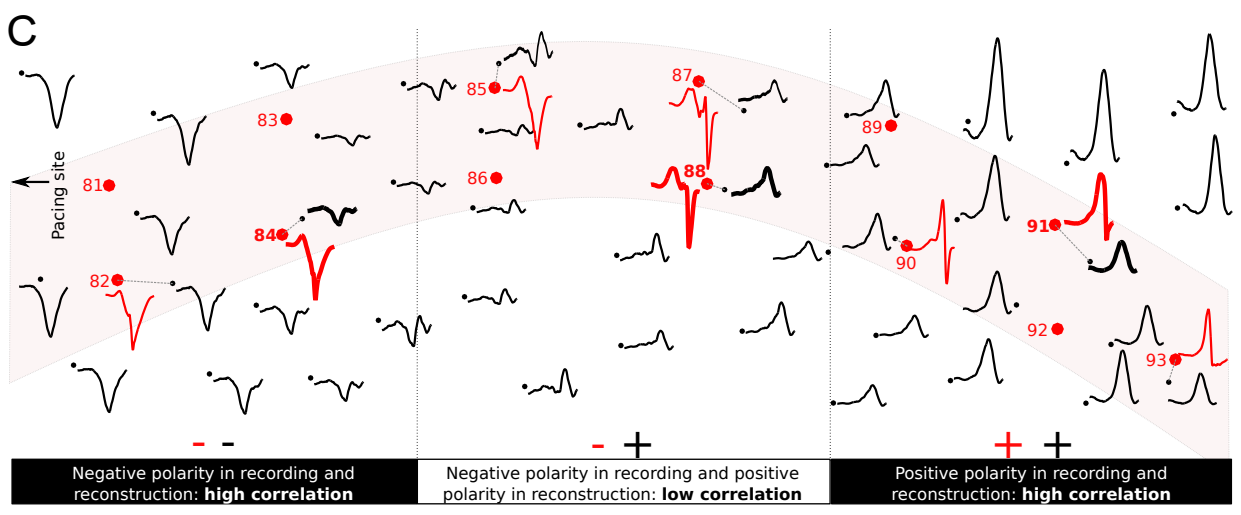

Figure 3.2: Paired examples of invasively recorded and noninvasively reconstructed electrograms, and activation and recovery times in a normal anesthetized dog. Spheres at the heart surface indicate the position of epicardial electrodes. For the numbered purple spheres, the recorded (red, left) and reconstructed (black, right) electrograms are depicted below. For each pair of electrograms, the correlation coefficient (CC) is given. Additionally, activation and recovery times are indicated with vertical green lines. The mismatch between the recorded and reconstructed time is given in ms at the right of each pair. The epicardial colors indicate noninvasive ventricular activation isochrones. Panel $\mathrm{A}$ shows a sinus beat, and panel $B$ a beat paced at LV epicardial electrode 75 (blue sphere). Panel $C$ zooms in on the region of electrodes 84-88-91 of the paced beat (only QRS complex shown), showing that the polarity change in the recorded electrograms (red) is more distal of the pacing site then in the reconstructed electrograms (black). 
Figure 3.2 also shows activation and recovery times in the recorded and reconstructed electrograms, defined as the moment of $\max -d V / d t$ (in the QRS complex) and max $d V / d t$ (in the T wave), respectively. The sinus beat (Figure $3.2 \mathrm{~A}$ ) shows early epicardial breakthrough at the anterior RV. The paced beat (Figure 3.2B) shows early activation at the location of pacing on the LV, and consequently late activation of the RV. For locations on the epicardium for which recorded electrograms were available, generally there was an activation time mismatch of only a few ms, unless the reconstructed electrogram deviated considerably from the recorded one (e.g., electrode 15 in Figure 3.2A). Overall, recovery times had a larger error than activation times.

Figure 3.3A shows the temporal correlation coefficients for different locations on the heart: LV anterior, lateral, posterior, RV lateral, and LV apical epicardium, respectively. Medians for the $\mathrm{LV}$ anterior and RV lateral regions were significantly different from the other regions $(p<0.05)$; however, the large overlap in data suggests that this is mainly an effect of the large sample size and has limited practical value. The correlation coefficient at the apex, determined from only a single electrode and not an ensemble of electrodes as the other regions, was significantly higher than at the other regions.

Figure 3.3 B shows the temporal correlation coefficients for the entire QRST complex and its different segments. Again, these were statistically significantly different, although due to the large overlap, these differences will have limited clinical value.

Figure $3.3 \mathrm{C}$ depicts the average spatial correlation between the recorded and reconstructed potentials for all epicardial electrodes, averaged for all 93 beats. In general, the spatial correlation decreased at the end of the QRS complex and the end of the T wave.

In Figure 3.3 D, spatial resolution of reconstructed electrograms is depicted. For each recorded electrogram, the distance was calculated between the recording electrode and the closest epicardial node that resulted in a good-enough correlation (coefficient $>0.70$ ). For $84 \%$ of all recorded electrograms, a good-enough correlation was found with a reconstructed electrogram at some location on the heart surface. For $90 \%$ of these electrodes, the distance to the closest epicardial node resulting in a good-enough correlation was less than $20 \mathrm{~mm}$.

\subsubsection{ACCURACY OF RECONSTRUCTED ACTIVATION AND RECOVERY TIMES}

Figure 3.3E shows the association between timings determined from the recorded and from the reconstructed electrogram. More accurate timings are closer to the identity line. Activation times as determined with the spatiotemporal approach (red dots, $R=0.82$ ) showed a stronger association $(p<0.05)$ than those determined with the temporal approach (gray dots in the background of the red dots, $R=0.73$ ). Recovery times were 
significantly more accurately $(p<0.05)$ determined after spatial smoothing (blue dots, $R=0.73$ ) than without (gray dots, $R=0.60$ ). Activation times could be determined more accurately than recovery times.

\subsubsection{LOCALIZATION OF BEAT ORIGIN}

One major application of ECGI has been its ability to localize the origin of ventricular extrasystolic (VES) beats and focal tachycardia. We have investigated this by noninvasively reconstructing the origin of beats that were paced by ventricular electrodes. The reconstructed location of earliest activation was compared to the known location of ventricular pacing for 80 beats in the 4 dogs. Each pacing location was included only once. Figure 3.4A shows that the median error of the temporal-only approach for activation timing was $33 \mathrm{~mm}$, with a range from 5-79 $\mathrm{mm}$. These results improved significantly by using the spatiotemporal approach, which resulted in a median error of only $10 \mathrm{~mm}$ (range 3$47 \mathrm{~mm}, p<0.05$ ). Also the range of errors decreased considerably: for the temporal-only approach, the origins of $90 \%$ of the beats lay within a $60 \mathrm{~mm}$ range from the known pacing location, whereas this range decreased drastically to $25 \mathrm{~mm}$ with the spatiotemporal approach.

We analyzed reconstructions of beats paced repeatedly at the same location, to investigate the stability of detection of the origin of these beats. For this purpose, 4 different pacing sites were investigated with in total 47 additional beats (these beats were not included in the previous analysis to avoid bias), Figure 3.4B. In general, all the detected origins (yellow spheres) for the same beat were within a confined region around the pacing location (blue sphere). Moreover, the median location (green sphere) of all detected origins had a lower mismatch than the mean mismatch of individual detected origins, demonstrating that it is worthwhile to reconstruct multiple instances of a monomorphic extrasystolic beat to obtain a more accurate location of its origin.

\subsubsection{CARDIAC MOVEMENT AND RECONSTRUCTION ACCURACY}

We refer to Section 3.7 of the Data Supplement at the end of this Chapter for the analysis of cardiac movement and reconstruction accuracy, where we show that there is a negative association between these quantities. The Data Supplement furthermore provides examples of canine cardiac activation and recovery patterns (Section 3.8), and provides an analysis of the endocardial or epicardial origin of paced beats (Section 3.9). To stimulate further research, data are freely shared (Section 3.6.6). 

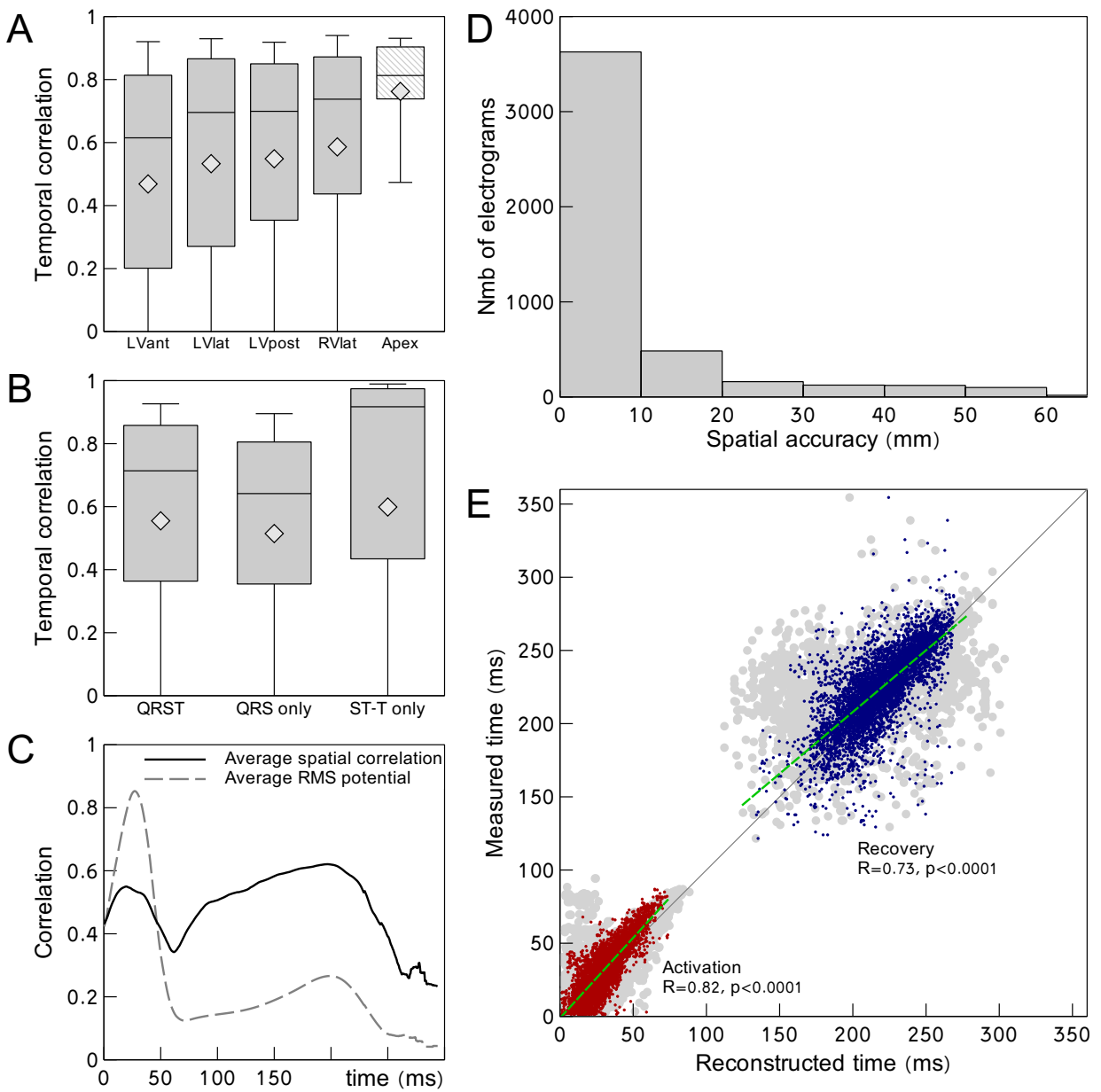

Figure 3.3: Accuracy of noninvasive reconstruction of epicardial potentials. Panel A: Temporal correlation coefficients for different locations on the heart: LV anterior/lateral/posterior sides, RV lateral side (all based on multiple electrodes) and apex (single electrode). Panel B: Temporal correlation coefficients for the full QRST segment of the cardiac beat (left), for only the QRS complex (middle) and only the ST-T segment (right) of all beats, respectively. Panel C: Spatial correlation coefficient per time instant (solid line), averaged over all electrograms. The root mean square (RMS) of the recorded epicardial potentials shows the average moment of the QRS complex (first peak) and T wave (second peak). Panel D: Histogram for recorded electrograms, showing the distance between the recording electrode and the closest virtual epicardial node which resulted in a good-enough correlation $(C C>0.70)$. Panel E: Activation (red dots) and recovery (blue dots) times as determined with the spatiotemporal approach from recorded electrograms (vertical axis) versus the timings determined from reconstructed electrograms (horizontal axis); gray dots in the background show the times as determined with the less accurate temporal-only approach. Timings are relative to QRS onset. Data collected over 93 beats in the 4 dogs (5552 pairs of electrograms). 

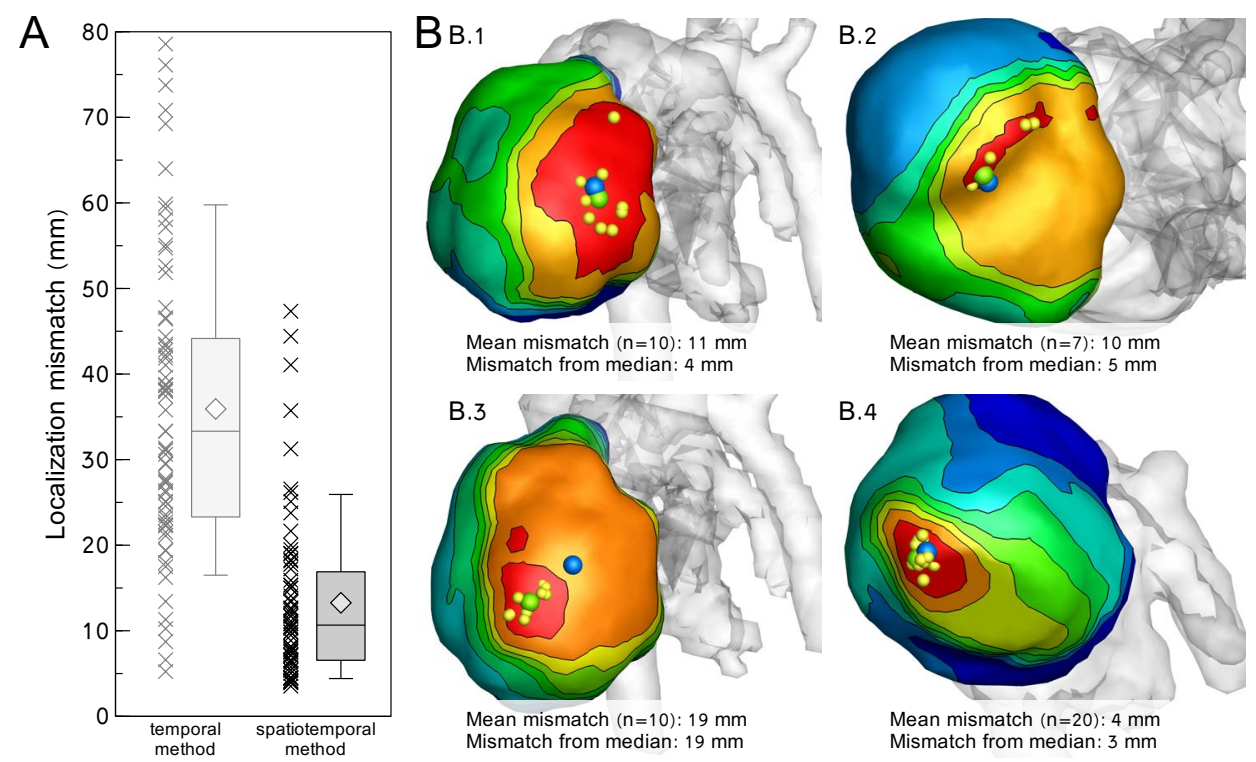

Figure 3.4: Accuracy of noninvasive localization of the origin of paced beats. Panel A: Scatter plots and box plots for localization mismatch with two different methods (temporalonly and spatiotemporal method) for 80 paced beats in 4 dogs. Localization mismatch is defined as the distance between the known pacing location and the location of earliest activation from noninvasively reconstructed epicardial electrograms. Panel B: Reconstructed origins of consecutive beats paced at the location indicated with the blue dot. Epicardial surface color indicates the reconstructed activation time for the first beat of that series (red: $0 \mathrm{~ms}$, isochrone lines drawn at $5 \mathrm{~ms}$ intervals). Yellow dots indicate the reconstructed origins for all beats of that series, all paced at the same location. The green dot is the median location of earliest activation and usually has a lower mismatch with the known pacing location than the mismatch of all beats.

\subsection{Discussion}

Noninvasive ECGI is increasingly applied in clinical settings. Table 3.1 summarizes recent validation studies that evaluated ECGI with quantitative data, e.g., from artificial torso tank setups or non-simultaneous invasive recordings in humans. In the present study, we acquired simultaneous recordings of body-surface and invasive epicardial potentials in normal anesthetized dogs under closed-chest conditions to compare reconstructed with recorded epicardial electrograms, activation/recovery timings and beatorigin localization. 
Medium, reference \& method

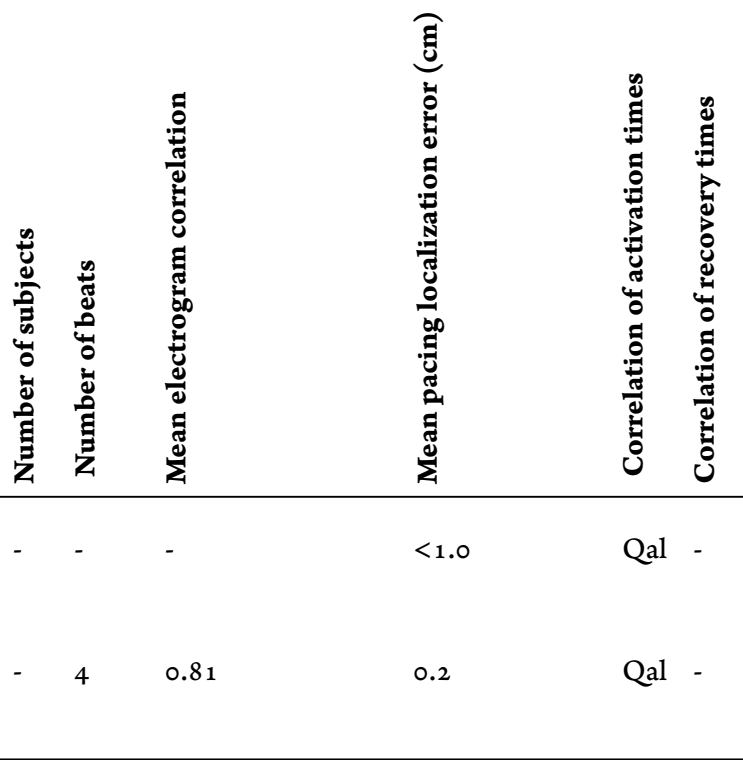

Torso tank [33]

Single site epicardial pacing in torso

tank

Torso tank [101]

Single and dual site epicardial pacing in torso tank

\section{Humans [78]}

Intraoperative mapping in patients

$\begin{array}{lllll}3 & 5 & 0.72 \pm 0.25^{*} & \sim 1.0 \quad \text { Qal - }\end{array}$

while pacing (nonsimultaneous recording, open chest)

Humans [101]

Ventricular pacing by implanted

$4 \quad 6$

0.5

pacemaker

Humans [44]

Endocardial atrial pacing in AF pa-

tients

\section{Humans [35]}

Epicardial ventricular pacing

Humans [57]

Pacemaker atrial/ventricular pacing

Humans [57]

Endocardial atrial/ventricular pacing

\section{Canines}

This study
$6 \quad 37$

$0.6 \pm 0.4$

$479 \quad-$

$1.3 \pm 0.9$

Qal -

$29456+$ -

$0.9 \pm 0.6$

$5412+$ -

$0.7 \pm 0.2$

Table 3.1: Overview of recent in vivo validation studies of the potential-based problem of ECGI. Only studies providing quantitative data (i.e., pacing location mismatch or invasive electrogram comparison) on ECGI validation were included. *Determined by allowing a time-shift (cross correlation); †Includes beats paced from identical locations (non-unique morphology); łmedian [IQR]; Qal = Qualitative agreement, no quantitative analysis performed. 


\subsubsection{VARIABLE ACCURACY OF RECONSTRUCTED ELECTROGRAMS}

Direct comparison of reconstructed and recorded electrograms showed that in general noninvasively reconstructed epicardial electrograms correlated well with the invasively recorded signals, with an overall median correlation coefficient of 0.71 . However, single electrograms could be discrepant, resulting in a lower correlation coefficient. For 90\% of all recorded electrograms for which an improved correlation was found, the distance to the closest epicardial node resulting in a good-enough correlation was less than 20 $\mathrm{mm}$. For $65 \%$ of the electrograms with a low correlation coefficient, the electrogram was in a region where surrounding electrograms changed from a morphology with a mainly positive to a mainly negative polarity (or vice versa). Combined, these results suggest that low correlations are primarily caused by a spatial mismatch between reconstructed and recorded electrogram. Although the spatial mismatch is generally small, it has a large effect on correlation coefficients in regions where the polarity of electrograms changes (Figure 3.2 C).

From Figure 3.2, it can also be seen that correlation coefficients did not always penalize relevant discrepancies between recorded and reconstructed electrograms. For example, epicardial electrogram 5 in Figure 3.2B exhibited an rS morphology, but the reconstructed electrogram missed this initial $r$ peak and showed a $\operatorname{Qr}$ pattern. These morphological differences were not reflected by the high correlation coefficient ( 0.92$)$, but could be clinically relevant when determining the origin of activation (see also Figure 3.7).

Our findings are in agreement with previous studies on noninvasively reconstructed epicardial electrograms in a torso tank and in humans, as can been seen from Table 3.1. In the previous torso-tank experiment, an explanted canine heart was suspended in a container with electrolyte solution. [101] Noninvasively reconstructed electrograms were compared to direct recordings on the epicardium, with a mean correlation coefficient (over 4 beats) of 0.81 . [101] The higher accuracy of that study might be explained by the lack of torso inhomogeneities (such as lungs), reduced breathing motion artifacts, and the distance of the recording electrodes to the epicardium (approximately $1 \mathrm{~cm}$ ). In the previous human study, non-simultaneous invasive recordings during open-heart surgery were compared to preprocedural noninvasive reconstructions. [78] These investigators showed a mean cross correlation of $0.72 \pm 0.25$ over 5 beats in 3 subjects. 'Cross correlation' is defined by shifting the reconstructed electrogram over the recorded one and computing the maximum correlation coefficient during this process. The results of that study should thus be compared to our 'time-shifted' correlation coefficients, as presented in Section 3.7 of the Data Supplement at the end of this Chapter. The mean values of time-shifted correlation coefficients $\left(\mathrm{CC}_{\text {mean }}=0.72\right)$ by Ghanem et al $[78]$ were 
comparable with our present ones $\left(\mathrm{CC}_{\text {mean }}=0.71\right)$. Our study extends these previous results by reporting medians and ranges, and by examining many more beats under closed-chest conditions, i.e., much closer to the non-invasive clinical situation.

Moreover, in the Data Supplement we show that there is a negative association between cardiac movement and reconstruction accuracy (Section 3.7). This should be investigated further, and it is to be expected that incorporating cardiac movement in ECGI will improve accuracy of reconstructed electrograms.

\subsubsection{SPATIOTEMPORAL APPROACH ENHANCES RECONSTRUCTION ACCURACY}

Accuracy of activation has been evaluated previously by qualitatively comparing activation isochrones in torso tank experiments and human studies, see Table 3.1. To the best of our knowledge, such an evaluation does not exist for recovery isochrones. We found that single electrograms could deviate considerably from the true (recorded) electrogram, and consequently, activation and recovery times could deviate considerably between reconstruction and recording. Noise, fractionation and reconstruction inaccuracies can lead to suboptimal results when using temporal-only criteria. By concatenating the characteristics of local electrograms as suggested by Erem et al [100], more robust interpretations were made, and this spatiotemporal approach led to high correlation coefficients $(R=0.82)$ for activation timings. Recovery timings attained slightly less ac$\operatorname{curacy}(R=0.73)$, which could be explained by lower amplitudes of the electrographic $\mathrm{T}$ wave, making it more sensitive to noise compared to the QRS complex. Additionally, when ignoring cardiac motion, the assumption of a static diastolic geometry has its largest negative impact on the reconstruction of the ST-T segment. Thus, activation time maps, and to a lesser extent recovery time maps, can be interpreted with confidence if a spatiotemporal algorithm is used.

\subsubsection{ECGI-BASED LOCALIZATION OF ECTOPIC BEATS IS ACCURATE WITHIN THE CM RANGE}

ECGI has been commonly applied to localize the origin of VES beats (see Table 3.1).The first study to investigate localization accuracy in humans found a precision of approximately $1 \mathrm{~cm}$, based on three paced beats. [78] In another study, ventricular pacing was performed in humans with simultaneous recording of body-surface potentials, and inverse reconstruction of the earliest site of activation had a median error of $1.3( \pm 0.9) \mathrm{cm}$ in healthy hearts. [35] In a similar study, an accuracy of $0.9( \pm 0.6)$ and $0.7( \pm 0.2)$ was obtained. [57] In atria, pacing sites could be localized with $0.6( \pm 0.4) \mathrm{cm}$ accuracy. [44] In the present study, we have shown that a spatiotemporal approach to determine the site 
of origin of paced beats resulted in a median error of $1.0 \mathrm{~cm}$. Importantly, the range of errors was limited, with $75 \%$ of the beats having an error less than $1.7 \mathrm{~cm}$, and $90 \%$ less than $2.5 \mathrm{~cm}$. These errors appear low enough for ECGI to support catheter-based diagnostic evaluation, thereby potentially improving therapeutic outcome. Currently, these results apply only to healthy hearts and are based on beats that were mostly paced from the epicardium.

This study was the first to investigate the reproducibility of beat localization and to show that noninvasive localization of pacing yields consistent and stable results. Another valuable aspect of VES localization would be to distinguish between an endocardial or epicardial origin. In the clinic, this could facilitate the choice between an endocardial or transthoracic epicardial catheter approach, reducing the need to convert from one to the other in specific cases. As we have demonstrated in the Figure 3.7, an endocardial origin gave an rS morphology on the epicardial electrogram.

\subsubsection{COMPARISON TO OTHER APPROACHES}

Many modeling choices are possible in ECGI. We have aimed at following common choices, but other approaches may yield better results. For example, we have applied Tikhonov zeroth order regularization, while other regularization methods may yield improved results in certain situations, e.g., the GMRes method might better distinguish between multiple pacing sites. [96] A method that employs meshless surfaces has been shown to be more accurate than when triangulated surfaces are used [102], as here. Furthermore, it has been shown that beat origin localization could be more accurate when not activation times are used, but the location of deepest negative potential during early activation. [103] Most of the studies from Table 3.1 use this minimal-potential approach, and not earliest-activation time. These different approaches in different laboratories illustrate the challenge in comparing and generalizing accuracies.

In this study, we investigated the potential-based formulation of the inverse problem. Originally, it was thought that this method could only lead to the reconstruction of epicardial potentials. Meanwhile, researchers have tried to reconstruct endocardial potentials as well. [82] These methods have not yet been investigated extensively or validated. A follow-up of this validation study could include endocardial potential recordings as well, to evaluate the accuracy of noninvasive reconstructions of endocardial potentials.

A radically different approach to the inverse problem is the wave-front formulation, which aims at reconstructing activation and recovery times directly, without intermediate reconstruction of cardiac potentials. [41] If one is only interested in activation and recovery times, this approach would have the theoretical advantage that by skipping 
the step of reconstruction of electrograms (with its inherent uncertainty), more reliable timings are obtained. However, no in vivo validation studies have been published, and these methods did not yet find widespread clinical application. We plan to compare the potential-based formulation and wave-front formulation in future research.

\subsubsection{LimitATIONS}

By using two electrode bands, there was no uniform recording coverage of the ventricles. However, a balance was achieved by positioning these bands at the basal and midbasal sections of the ventricles (covering the circumferentially largest parts of the ventricles) and an additional electrode at the apex. Although an electrode sock might have provided more uniform electrode coverage, our approach offered more flexibility to different heart sizes and shapes, and did not constrain cardiac mechanics. Nevertheless, the apical area was underrepresented. Reconstruction accuracy could be different at the apex than at the basal and midbasal areas of the heart due to the closer proximity of the apical area to the body-surface electrodes. The single electrode at the apex achieved a significantly higher correlation for reconstructed electrograms than the other epicardial regions. This suggests that our results, which are based mainly on recordings in basal and midbasal regions, represent the lower bounds of reconstruction accuracy of the full heart.

Implantation of electrodes, contained by silicone bands, could alter local conductivity and propagation of electrical signals to the torso. Bear et al [104], however, did not find any difference when comparing body-surface electrograms before and after electrode implantation in their recent study.

We investigated a large diversity of rhythms: sinus, atrially paced, and endocardial and epicardial ventricular paced rhythm, with rates ranging from 85 to $143 / \mathrm{min}$. We did not investigate atrial or ventricular fibrillation, which is much more complex and most likely more difficult to reconstruct.

To comply with most commonly used ECGI implementations, we have chosen a torso-heart geometry that did not contain any inhomogeneities with distinct conductivity, such as lungs, fatty tissue and bones. Although these inhomogeneities may alter propagation of electromagnetic fields, their inclusion is time consuming and increases complexity of the inverse reconstruction, making it more sensitive to noise.

\subsection{Conclusions}

This validation study addressed the accuracy of electrogram reconstructions for normal and abnormal electrical activity in intact anesthetized dogs, filling the gap between pre- 
vious experiments with explanted hearts and ECGI application in humans. We have demonstrated that noninvasive reconstruction of epicardial potentials is generally attained at a high median accuracy, but with considerable spread. Overall, the spatial resolution was far smaller than $20 \mathrm{~mm}$, and outliers were mainly due to a spatial mismatch between recording and reconstruction in regions of electrographic transition. Given this variation, our results on activation and recovery timing were most reliable when the characteristics of neighboring electrograms were incorporated as well. This spatiotemporal approach allowed for accurate and consistent reconstruction of beat origins (median error $10 \mathrm{~mm}$; $90 \%$ within $25 \mathrm{~mm}$ ), which, upon translation, could expedite catheter-based diagnostic evaluation and ablation in clinical settings. The results presented in this Chapter support adequate applications of ECGI at the ventricles during regular and abnormal rhythm. Cardiac motion was negatively associated with reconstruction accuracy, and accounting for cardiac motion in future ECGI algorithms could yield further improvements.

\section{ACKNOWLEDGMENTS}

The authors would like to thank Roel Spätjens, BSc, for help with the figures, and Lars van Middendorp, $\mathrm{MD}, \mathrm{PhD}$, Marc Strik, $\mathrm{MD}, \mathrm{PhD}$, and Marion Kuiper, BSc, for their invaluable support in the animal experiments. Furthermore, we would like to thank Dana Brooks, PhD, Jaume Coll-Font, MSc (Northeastern University, Boston, MA, USA), and Burak Erem, PhD (Harvard Medical School and Boston Children's Hospital, MA, USA), for kindly sharing their algorithm for spatiotemporal activation time approximation.

\section{Disclosures}

None. 


\section{Data Supplement to Chapter 3}

In this appendix to Chapter 3 , we discuss the following:

1. Details on the experimental setup, data collection, inverse reconstruction and data post-processing;

2. Analysis of the association between cardiac motion and reconstruction accuracy;

3. Examples of activation and recovery patterns in canines;

4. Analysis of reconstructed epicardial electrograms to distinguish between endocardial versus epicardial beat origins.

\subsection{Detailed methods}

\subsubsection{ANIMAL EXPERIMENTS}

Four normal dogs were included. Midazolam i.v. ( $0.25 \mathrm{mg} / \mathrm{kg} / \mathrm{h}$ ) and sufentanil i.v. $(3 \mu \mathrm{g} / \mathrm{kg} / \mathrm{h})$ were used as anesthetic agents. The animals were ventilated with $30 \% \mathrm{oxy}$ gen in pressurized air to normocapnia, with continuous monitoring of oxygen saturation (kept at 98-100\%) and ventilation pressure $\left(15-20 \mathrm{~cm} \mathrm{H}_{2} \mathrm{O}\right)$.

Two silicone bands with 99 electrodes were implanted around the basal and midbasal ventricular epicardium after thoracotomy. Each band consisted of two rows of electrodes. The electrodes of the basal band were made of silver and spaced $10 \mathrm{~mm}$ in both directions; the electrodes of the apical band were made of stainless steel and spaced $10 \mathrm{~mm}$ in the direction of the band, and $5 \mathrm{~mm}$ in the perpendicular direction. Each electrode was $2 \mathrm{~mm}$ in size. Additional electrodes were placed at the left ventricular (LV) apical epicardium, the LV endocardium (via a plunge electrode introduced through the myocardium), the right ventricular (RV) apical endocardium (via an endovascular catheter tip), and the right atrial endocardium (also by catheter). Invasive electrodes and recording system were developed by Instrument Development Engineering \& Evaluation (IDEE), Maastricht, the Netherlands. After airtight closure of the chest, residual air was removed from the thorax with a vacuum system. Body-surface electrodes (on average 200, range 184-216; ActiveTwo Setup from BioSemi, Amsterdam, the Netherlands) were attached to the torso, with a reference electrode on a hind leg. Unipolar potential recordings were obtained simultaneously at the epicardial electrodes (sampling frequency of $1000 \mathrm{~Hz}$ ) and body-surface electrodes (sampling frequency of $2048 \mathrm{~Hz}$ ). A helical ECG-gated CT scan was performed in a Siemens Somatom Definition Flash scanner with intravenous iodine contrast. CT voxel dimensions were $0.70 \times 0.70 \times 0.50$ $\mathrm{mm}$.

Potential recordings were notch-filtered to reduce powerline interference. 


\subsubsection{INVERSE RECONSTRUCTION}

Data analysis was performed in the mathematical computing package MATLAB. [21] A triangulated torso-heart geometry was digitized from the CT scan and consisted of the body-surface electrodes, and the epicardial surface (consisting of on average 1693 nodes); additionally, the positions of the 103 implanted electrodes were digitized. This digitization was performed manually from the CT scans, with the $\mathrm{Seg}_{3} \mathrm{D}$ software. [18] The torso-heart geometry did not include any torso inhomogeneities (such as lungs, bones or fat tissue). The transfer matrix, relating the potentials at the body surface to the epicardial surface, was computed from the torso-heart geometry using publicly available methods. [19]

Direct inverse reconstruction of epicardial potentials from the measured bodysurface potentials suffers from uncertainty due to the ill-posed character of the inverse problem. [99] Therefore, the commonly used Tikhonov zeroth order regularization was applied to obtain epicardial potentials. [93] The regularization parameter was automatically determined using the L-curve method. [20] We refer to 'nodes' as the virtual points on the epicardial surface on which potentials were reconstructed, and to 'electrodes' as the physically implanted electrodes by which epicardial potentials were recorded.

\subsubsection{POST-PROCESSING TO OBTAIN ELECTROGRAMS, ISOCHRONES AND ACTIVATION ORIGIN}

Electrograms were reconstructed per epicardial node by concatenating potentials over time. Activation times were determined per electrogram with the temporal-only and spatiotemporal method. The temporal-only approach defined the moment of activation simply as the moment of steepest voltage down slope (maximum $-d V / d t$ ) during the QRS complex. The spatiotemporal approach, proposed by Erem et al, takes advantage of the spatial relationship between neighboring nodes and their potentials, and might be better suited to estimate the activation time in noisy or fractionated electrograms. [100] They note that not only the temporal signal (local potential at a single node) changes quickly when an activation wavefront passes, but also the spatial gradient of potentials between neighboring nodes. Their approach to activation time estimation selects the time point that matches the change in temporal derivative with the change in spatial derivative. More formally, for each epicardial node, they define the activation time $\tau$ as:

$$
\tau=\min _{t}\|D V(t)\|_{2} \cdot \frac{\partial V(t)}{\partial t}
$$


where $V(t)$ is the potential at the epicardial node under consideration at time $t, D V(t)$ is the approximated spatial gradient, and $\partial V(t) / \partial t$ the approximated temporal derivative.

Recovery times were defined as the moment of maximum $d V / d t$ during the $\mathrm{T}$ wave. To deal with outliers, some spatial smoothing was applied to activation and recovery times at the virtual epicardial surface; however, no smoothing was applied on the times determined from the measured epicardial electrograms. Location of earliest activation was defined as the epicardial node with the earliest activation time. QRS and T-wave delineation was performed manually per beat.

\subsubsection{Statistical analysis}

For each epicardial electrode, Pearson's correlation coefficient (CC) was computed between the recorded electrogram and the reconstructed electrogram at the corresponding (closest) virtual epicardial node. For this purpose, the epicardial recordings (obtained at $1000 \mathrm{~Hz}$ ) were upsampled to match the epicardial reconstructions (obtained at 2048 $\mathrm{Hz}$ ). Alignment of the epicardial and body-surface recordings was achieved by matching the timing of pacing spikes. Linear correlation between measured and reconstructed activation/recovery timings was assessed by means of Pearson's correlation coefficient R. Statistical comparison of correlation coefficients and activation and recovery timings was achieved by Wilcoxon signed-rank (for paired measurements) or Wilcoxon rank-sum (for unpaired measurements) tests.

\subsubsection{Polarity CHANGe}

For each of the recorded electrograms with a correlation coefficient lower than 0.40 , we investigated whether this electrode was in an area where the electrograms changed from a positive to a negative polarity or vice versa (as a sign of electrographic transition). This was assessed by determining the sign of the mean amplitude of the electrograms (with sufficient correlation coefficient) of 8 electrode pairs 'downstream' on the electrode band, and the sign of the electrograms of 8 electrode pairs 'upstream' (left and right in Figure 3.2C). When the downstream signs where opposing the upstream signs, this confirmed that the electrode with low correlation coefficient was in a region of polarity change. Note that this method cannot detect such regions if the polarity change is not in the upstream/downstream direction, but perpendicular to the electrode bands. 


\subsubsection{SHARING OF DATA}

Methodology and validation studies of ECGI have been conducted for decades. Ethical and practical limitations have made it difficult to simultaneously obtain body-surface potential recordings and extensive intracardiac recordings in physiological realistic settings. To stimulate further research, a subset of the validation data presented in this study is shared freely (see http://mecgi.org/). [105] Recently, also other attempts on datasharing have been initiated by the creation of the Consortium for ECG Imaging. [106] It is expected that these initiatives will help to improve noninvasive reconstruction of electrical cardiac activity even further.

\subsection{INFLUENCE OF CARDIAC MOTION ON RECONSTRUCTION ACCURACY}

Currently, all applications of inverse ECGI known to us assume a heart geometry that is static, which ignores the potential influence of mechanical motion of the heart during its beating. We investigated whether there was a correlation between mechanical motion and reconstruction accuracy.

Full helical CT scans were obtained during normally-conducted sinus beats, to determine the digital epicardial geometry for each $10 \%$ of the RR interval. Thus, we could track the movement of epicardial electrodes during the cardiac cycle. Accuracy was limited by the voxel dimensions of the CT scan, being $0.70 \times 0.70 \times 0.50 \mathrm{~mm}$, and the relatively high heart rate ( \pm 115 beats per minute). Epicardial electrograms were reconstructed assuming a static heart geometry obtained during late diastole. Figure 3.5A shows three pairs of recorded and reconstructed electrograms, and Figure $3.5 \mathrm{~B}$ the corresponding electrode movement for each segment of the RR interval. As can be seen, the larger the total movement of an electrode during the cardiac cycle, the larger the mismatch between recorded and reconstructed electrograms. Figure $3.5 \mathrm{C}$ shows this association for all epicardial electrodes during a sinus beat, confirming a lower quality of reconstruction with larger movement of the epicardium.

To investigate the influence of movement on reconstruction quality in all beats, we investigated the hypothesis depicted by the cartoon inserted in Figure 3.5D. If an electrode moves during a beat and this movement is not accounted for by a change in the digitized heart geometry, the electrogram recording could have moved away from the activation wave front, resulting in a recorded electrogram (' $x$ ') with a later activation peak than the electrogram reconstructed at its assumed position ('o'). If, on the other hand, the electrode would have moved towards the wave front, the recorded electrogram would show earlier activation. We investigated this by time-shifting the reconstructed electro- 
gram of each electrode by $\pm_{5} \mathrm{~ms}, \pm 12.5 \mathrm{~ms}$ and $\pm_{25} \mathrm{~ms}$, and computing the highest correlation coefficient during this process. This is also known as computing the maximum cross correlation between pairs of electrograms. Indeed, as shown in Figure 3.5D, determining the maximum correlation within a certain time window results in a significantly higher median correlation coefficient for all paced and non-paced beats, from 0.71 (interquartile range (IQR): 0.36-0.86) without a time-shift to 0.82 (IQR: 0.59-0.92) with a time-shift of maximally $25 \mathrm{~ms}$ per electrode.

Ghanem et al [78] reported correlation coefficients for reconstructed epicardial potentials in humans based on non-simultaneous recordings of body-surface and epicardial potentials during intrathoracic surgery. Interestingly, the correlation coefficients reported in that study were obtained after allowing a time-shift to compensate for misalignment of the signals that were acquired non-simultaneously and under different conditions. This should have resulted in a time-shift that was equal for all electrograms of the same beat. However, this was not the case. These time-shifts could be considered to indicate that cardiac motion and deformation recovery mechanics influenced reconstruction accuracy.

Thus, we have shown for the first time in vivo that cardiac motion was associated with lower accuracy of electrogram reconstructions. When a time-shift was allowed, correlation coefficients increased considerably, indicating that spatial movement - when not accounted for - might result in a time-shift of the reconstructed electrogram compared to the recorded one. However, in clinical applications it would be challenging to predict the extent and direction of the time-shift per electrogram owing to cardiac motion. Furthermore, uncorrected spatial movement would not only result in a time-shift, but also in morphological changes of the electrogram, as illustrated in Figure 3.5A. Future studies should show that incorporating cardiac movement in the inverse problem leads to actual improvements in reconstruction accuracy. This could be achieved by capturing cardiac motion with MRI or CT with ECG-gated dose modulation.

Additional motion effects due to breathing (both movement of the torso surface, and movement of the heart within the torso) were not taken into account in our analyses, but likely influence reconstruction quality as well.

\subsection{ACTIVATION AND RECOVERY PATTERNS}

Figure 3.6 shows the activation and recovery patterns for several beats. For a sinus beat (panel A), earliest epicardial breakthrough occurred predominantly at the superior RV 

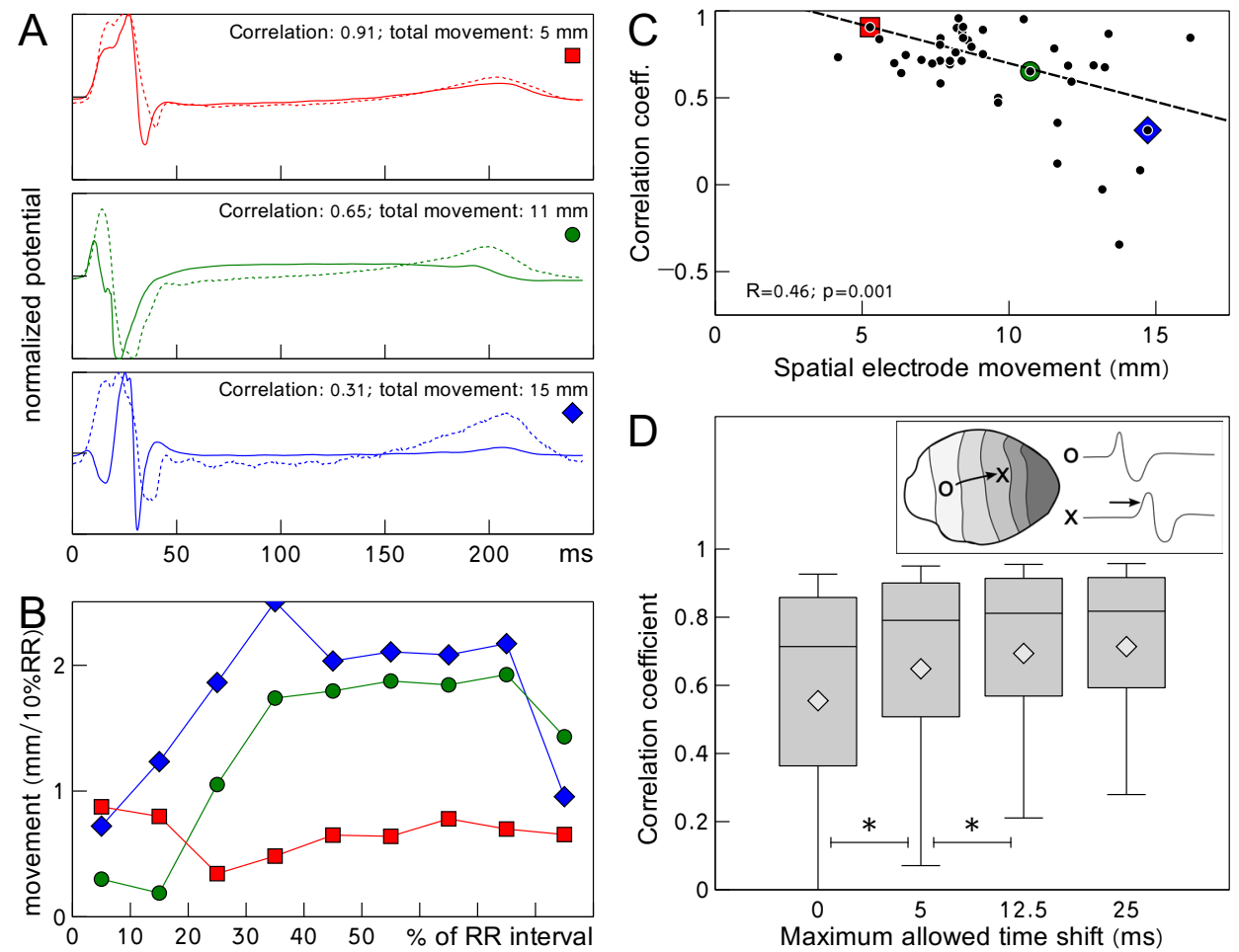

Figure 3.5: Association between reconstruction accuracy and mechanical motion of the heart. Panel A: Recorded (solid line) and reconstructed (dashed line) electrograms for epicardial electrodes that show only little (top), moderate (middle) or much movement (bottom) during a sinus beat. Panel B: movement of these electrodes per $10 \%$ of the RR cycle during a sinus beat, as determined from a CT scan. Panel C: Association between reconstruction quality and electrode movement. Linear regression fit is shown. In general, more electrode movement results in lower reconstruction quality. This is confirmed in panel $D$ for all beats in all dogs, where we investigated whether uncorrected spatial movement results in a temporal shift in the reconstructed electrogram (inset). The left box plot shows the correlation coefficient for 5552 pairs of electrograms when the reconstructed electrograms are directly compared to the recorded electrograms (box spans the interquartile range (IQR), i.e., the $25-75 \%$ range; median indicated by horizontal line; mean indicated by diamond; whiskers at $9-91 \%$ range). In the other box plots, each reconstructed electrogram is allowed to shift maximally $\pm 5, \pm 12.5$ or \pm 25 ms to obtain a maximum correlation for that electrogram. *, $p<0.05$. 

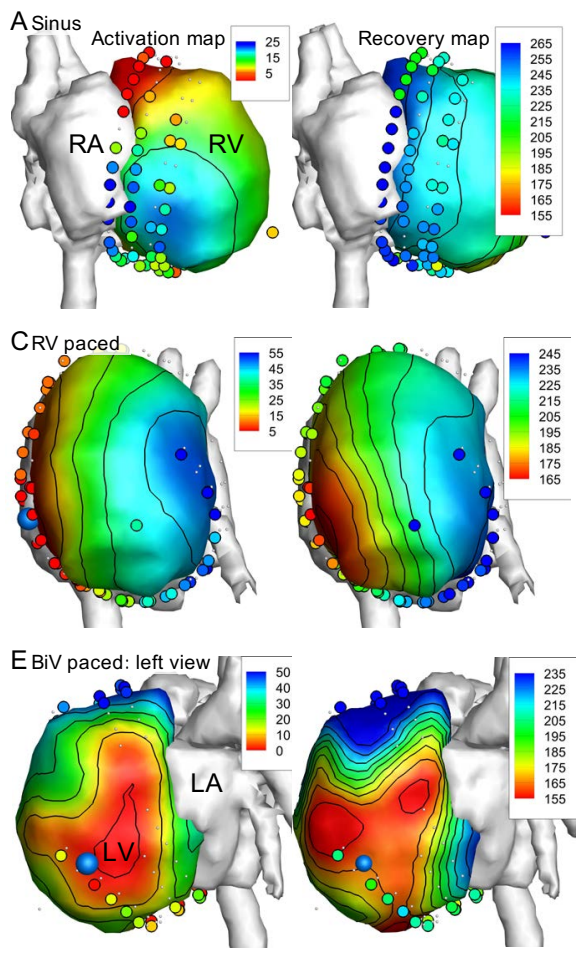
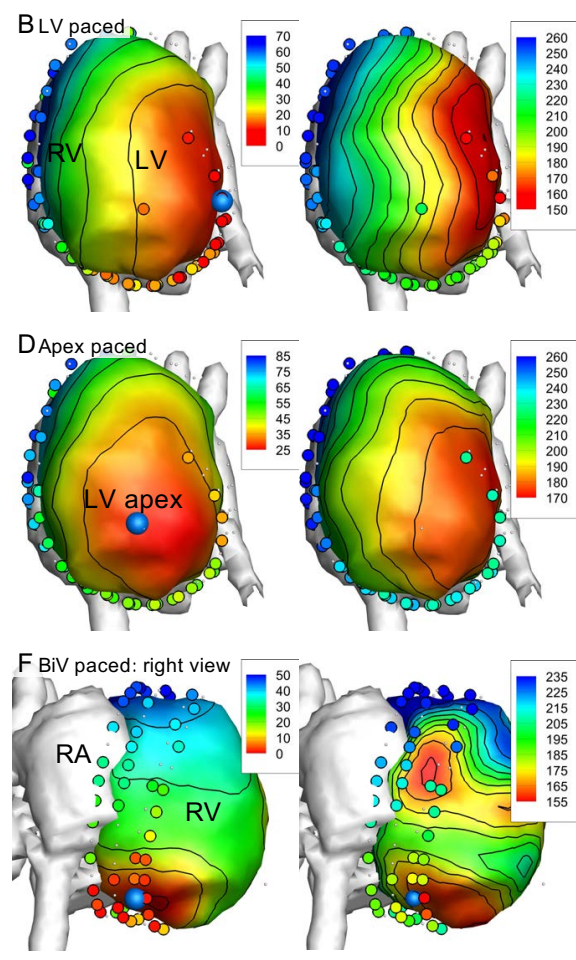

Figure 3.6: Pairs of reconstructed activation (left) and recovery isochrones (right) for multiple beats. Panel A: sinus beat; B: pacing at the left ventricle (LV); C: pacing at the right ventricle (RV); D: pacing at the LV apex; E \& F: left and right view for a beat paced simultaneously at the LV and RV (BiV pacing). Pacing locations are at the epicardium and indicated with the blue spheres. Measured activation and recovery times at electrode positions are indicated with the colored circles; colors correspond to the same scale as the reconstructed isochrones. In general, recovery followed a similar pattern as activation, and for the biventricularly paced beat, two distinct areas of first activation were reconstructed.

free wall, near the septum, followed by rapid activation of the rest of the tissue. Recovery, however, was much more homogeneously distributed and followed a different (more gradual) pattern than activation.

During pacing, activation was much slower than during sinus rhythm. Beats paced at the LV (panel B), RV (panel C) and LV apex (panel D) all showed much slower and gradual activation, indicating that the conduction system is not involved in this process. Total epicardial activation during paced rhythm (duration: 50-70 ms) lasted about twice as long as during sinus rhythm $(25 \mathrm{~ms})$. Epicardial recovery in paced beats followed the pattern of activation very closely. Duration of recovery was more or less similar for paced and native beats. Typically, recovery started around $160 \mathrm{~ms}$ after first epicardial activa- 
tion, and ended at $260 \mathrm{~ms}$.

It is important to note that activation duration is the time interval between the earliest and latest maximum $-d V / d t$ and not the total duration of electrogram change. The duration of electrogram change is typically longer, as this runs from the very first change in morphology (which occurs before the earliest maximum $-d V / d t$ ) until the very last change (after the latest maximum $-d V / d t$ ). For example, in sinus rhythm the epicardial activation duration was around $25 \mathrm{~ms}$, whereas the duration between the earliest and latest detectable change over all reconstructed electrograms was $45 \mathrm{~ms}$, and body-surface QRS width was $48 \mathrm{~ms}$. For the beat paced at the apex, epicardial activation duration was around $60 \mathrm{~ms}$, duration between earliest and latest epicardial electrogram change $71 \mathrm{~ms}$, and body-surface QRS width $78 \mathrm{~ms}$.

Panels $\mathrm{E}$ and $\mathrm{F}$ show the left and right view of the heart for a beat that was paced simultaneously at two epicardial locations: at the RV, and at the LV, i.e., biventricular $(\mathrm{BiV})$ pacing. Clearly, two distinct locations of early activation could be discerned. The activation wave fronts fused approximately at the inferior midline between the ventricles, with late activation of the superior basal side of the heart.

\subsection{ENDOCARDIAL VERSUS EPICARDIAL PACING}

Endocardial pacing yielded activation that was different compared to epicardial pacing, as demonstrated by panel $\mathrm{A}$ in Figure 3.7. In these experiments, the beats were either paced endocardially (left column), or at the corresponding location on the epicardium (right column), perpendicular to the ventricular wall. Although epicardial activation patterns were globally similar for both pacing locations, endocardial pacing resulted in faster activation than epicardial pacing.

It is worthwhile to determine from epicardial reconstructed electrograms whether the initiation was on the endocardium or epicardium. Reconstructed epicardial electrograms at the location of earliest activation are shown in Figure $3.7 \mathrm{~B}$. The epicardial electrogram showed a positive deflection (marked with an asterisk) before the negative deflection ( $r S$ morphology) for endocardial pacing, as the result of the activation wavefront approaching from the endocardium. Such a deflection appeared absent when epicardial pacing was applied (although the pacing artifact prevented a thorough analysis). This indicates that one could distinguish between endocardial and epicardial initiation from the reconstructed epicardial electrogram in case of an $\mathrm{rS}$ morphology or S morphology, respectively. 


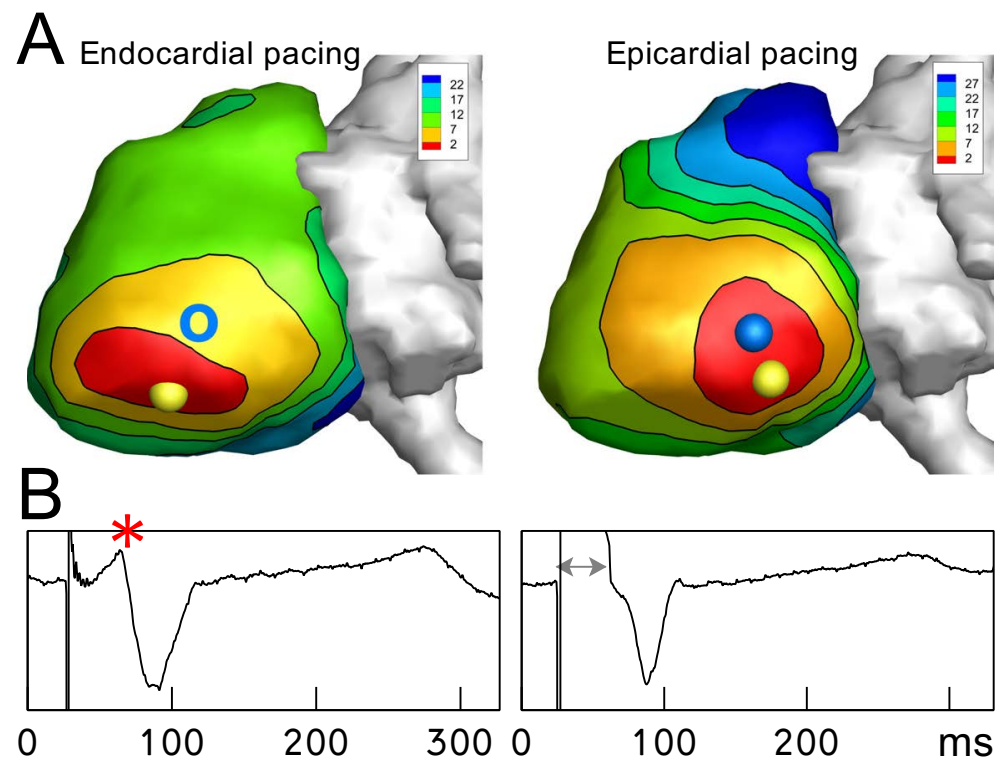

Figure 3.7: Reconstructed isochrones and electrograms for an endocardially paced beat (left column: pacing location endocardially from the blue circle) and an epicardially paced beat (right column: pacing location at the blue sphere) on the LV. Panel A indicates similar activation patterns for either pacing location, although endocardial pacing spread faster. Panel B shows the reconstructed epicardial electrograms at the location of reconstructed earliest activation (yellow sphere). The potential in the time period indicated with the doubleheaded arrow is the pacing artifact. The presence or absence of a positive initial deflection (marked with an asterisk) could be used to indicate the endocardial or epicardial origin of a beat, respectively. 


\begin{abstract}
The inverse problem of electrocardiography aims at noninvasively reconstructing electrical potentials on the heart surface from recorded body-surface electrocardiograms. A crucial step is regularization, which deals with ill-posedness of the problem by imposing constraints on the possible solutions. We developed a regularization method that includes electrophysiological input. Body-surface potentials are recorded and a computed tomography scan is performed to obtain the torso-heart geometry. Propagating waveforms originating from several positions at the heart are simulated and used to generate a set of basis vectors representing spatial distributions of potentials on the heart surface. The real heart-surface potentials are then reconstructed from the recorded body-surface potentials by pursuing a sparse representation in terms of this basis. This method, which we named 'physiology-based regularization' (PBR), was compared to traditional Tikhonov regularization and validated using in vivo recordings in dogs. PBR recovered details of heart-surface electrograms that were lost with traditional regularization, attained higher correlation coefficients and led to improved estimation of recovery times. The best results were obtained by including approximate knowledge about the beat origin in the PBR basis. These advances can improve risk stratification for patients at risk of sudden cardiac death.
\end{abstract}

Adapted from: MJM Cluitmans*, M Clerx*, N Vandersickel, RLM Peeters, PGA Volders, RL Westra. Physiology-based regularization of the electrocardiographic inverse problem. In revision.

* The first two authors contributed equally to this work: The PBR-method was developed and tested by Matthijs Cluitmans, who also collected the in vivo data. All AP simulations were set up and run by Michael Clerx. Both contributed equally to the text of the final publication. 


\subsection{INTRODUCTION}

As discussed in the previous Chapters, electrocardiographic imaging (ECGI) aims at noninvasively reconstructing the electrical activity of the heart, based on body-surface potential measurements and a patient-specific torso-heart geometry [14], [34], [38], [99], [107]. This is achieved by solving what is known as the inverse problem of electrocardiography. In the last decades, much progress has been made in ECGI and clinical applications are published with increasing frequency, yet the accuracy of the reconstructed electrical heart activity is still suboptimal. This is partly due to the ill-posedness of the inverse problem: small variations (noise) in the input data will yield unique but unrealistic variations in the reconstructions, see Chapter 2. To cope with this problem, regularization is applied, i.e., additional knowledge is incorporated in the form of constraints on the possible solutions to attain more realistic results. Such constraints are often based on physical or mathematical properties of the problem [36], [108], but the use of electrophysiological properties has also been proposed [109], [1 10$]$.

In a previous study [109], we have shown that it may be beneficial to also include simulated electrophysiological input in the reconstruction process, using a method we call physiology-based regularization (PBR). In this method, propagating waveforms originating from several positions on the heart are simulated and used to generate a set of basis vectors representing spatial distributions of potentials on the heart surface. The real heart-surface potentials are then reconstructed from the recorded body-surface potentials by pursuing a sparse representation in terms of this basis.

In this Chapter, we provide a detailed description of the PBR method and use in vivo recordings to assess its performance. We investigate whether PBR can improve reconstructions of epicardial ventricular potentials, specifically with the goals of detecting the origin of ectopic beats and imaging substrates for arrhythmias.

\subsection{Methods}

The potential-based formulation of the forward/inverse problems of electrocardiography is based on the assumption that there is a direct relation between potentials on a closed surface surrounding the heart, and the body surface [82]. The closed surface surrounding the heart is usually taken to be the epicardium, i.e., the outer myocardial layer. The forward problem can then be defined as:

$$
\Phi_{B}(t)=A \Phi_{H}(t)
$$


where $\Phi_{B}(t)$ are the potentials on the body surface at a specific time instant $t, \Phi_{H}(t)$ the potentials on the heart surface, and $A$ is the transfer matrix that relates these vectors. The transfer matrix captures the geometry and conductivity relation between the surfaces. It is assumed that the problem is quasi-static and the torso volume is source-free, and, therefore, the transfer matrix is time-independent.

The goal of the inverse problem is to find the cardiac potentials $\Phi_{H}(t)$ from recorded body-surface potentials $\Phi_{B}(t)$ and a patient-specific transfer matrix $A$ (usually based on a computed tomography (CT) scan). However, small variations in the body-surface potentials, e.g., due to noise, will result in disproportionately large changes in the computed cardiac potentials. In other words, the computed solution of the inverse problem does not depend continuously on the data and the problem is, therefore, ill-posed. Additional constraints are needed to obtain a stable, regularized solution. For example, the wellknown Tikhonov method obtains a stable solution by placing bounds on the amplitude of the reconstructed cardiac potentials (or derivatives thereof) with a least-squares minimization at time instant $t$ :

$$
\min _{\Phi_{H}(t)}\left\{\left\|A \Phi_{H}(t)-\Phi_{B}(t)\right\|_{2}^{2}+\lambda(t)\left\|R \Phi_{H}(t)\right\|_{2}^{2}\right\}
$$

where $R$ is the regularization operator (the identity matrix for zeroth order, the gradient operator for first order, or the Laplacian for second order regularization). The regularization parameter $\lambda(t)$ balances the quality of fit with the amount of regularization and can be determined with methods such as the L-curve. Recent papers review these and other regularization methods [82], [98].

PBR is a regularization method that constrains the solutions based on patientspecific electrophysiology data. It is illustrated schematically in Panel A of Figure 4.1. First, body-surface potentials are recorded and a CT scan is performed from which both a digitization of the heart-surface geometry and the location of the body-surface electrodes are obtained. Next, simulations of propagating action potentials (APs) originating from different points on the digitized epicardium are run. Singular value decomposition (SVD) is applied to the simulated patterns of epicardial potentials, and truncation is applied to arrive at a small set of basis vectors representing spatial distributions of potentials on the heart. The real epicardial potentials are then reconstructed as sparse combinations of these vectors. To validate this method, we performed in vivo measurements of the epicardial potentials in three normal, anesthetized dogs, while simultaneously recording potentials at the body-surface. This is illustrated in Figure 4.1, Panels B and C. 

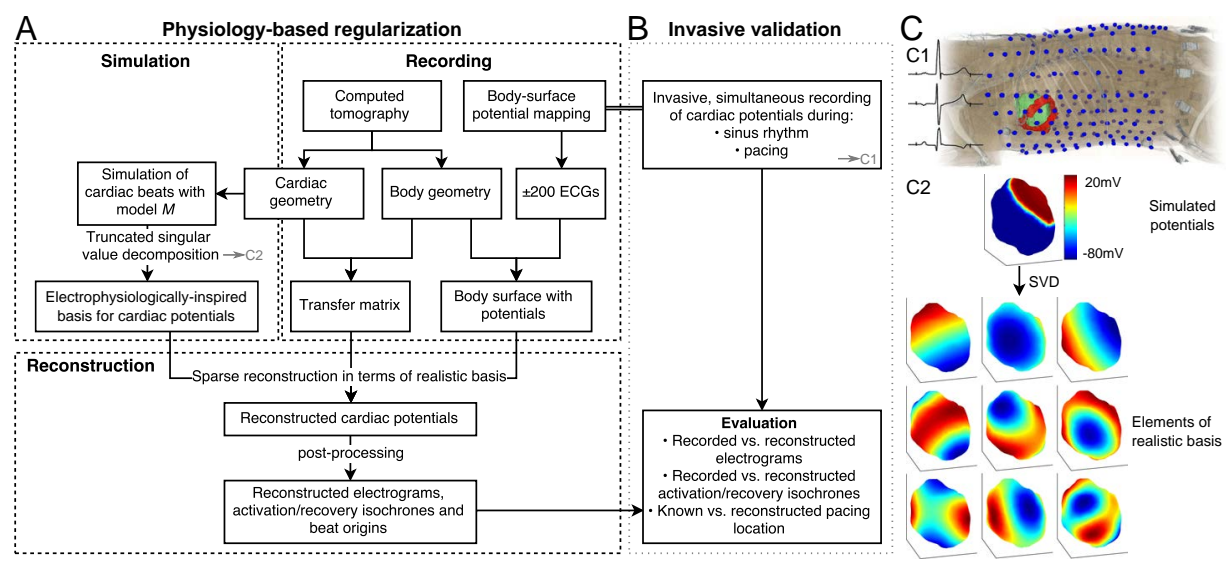

Figure 4.1: Schematic representation of physiology-based regularization (Panel A) and its validation in an in vivo canine model (panel B). Panel C1: Body-surface electrodes (blue), ventricular epicardium (green) and epicardial electrodes (red) in the canine experiment. Panel C2: Simulated potentials on the epicardium. Taking the singular value decomposition (SVD) of many (morphologically distinct) simulated beats yields a realistic basis of electrophysiologically relevant solutions that will be used to reconstruct epicardial potentials.

\subsubsection{IN VIVO RECORDINGS}

In vivo data was acquired in experiments with three out of four of the anesthetized dogs as discussed in Chapter 3. In short, in three normal anesthetized dogs, two silicone bands with 99 electrodes were implanted around the basal and mid-basal epicardium after thoracotomy. Each band consisted of two rows of electrodes. Additional electrodes were placed at the LV apical epicardium, the LV endocardium, the RV apical endocardium, and the right atrial endocardium, providing a total of 103 electrodes. After chest closure, body-surface electrodes ( 184 to 216 , depending on torso size) were attached to the chest. This number of body-surface electrodes is more than sufficient to obtain a good reconstruction with traditional methods.[1 11 l] Unipolar potential recordings were obtained simultaneously by the epicardial and body-surface electrodes.

Beats were recorded during normal sinus rhythm or with epicardial pacing from electrodes on the left ventricle (LV) or right ventricle (RV).

\subsubsection{TORSO-HEART GEOMETRY}

A CT scan was performed and used to digitize a homogeneous geometry consisting of the body-surface electrodes and the epicardial surface. Segmentation of the surfaces from CT scans was performed manually with $\mathrm{Seg}_{3} \mathrm{D}[18]$. The ventricular epicardium was digitized as a triangulated mesh with approximately 1700 nodes and the position of the 103 
implanted electrodes was recorded. The transfer matrix, relating the electrical activity on the cardiac surface to the body surface, was computed with methods available from the SCIrun software repository [19] and was based on a boundary-elements method. In one dog a three-dimensional digitization of the entire ventricular myocardium was created in addition to the epicardial surface. This digitization had a much finer resolution, with grid points spaced $0.5 \mathrm{~mm}$ apart in the $\mathrm{x}, \mathrm{y}$ and $\mathrm{z}$ directions.

\subsubsection{SimUlation OF EPICARDIAL POTENTIALS}

Computational models of the AP were used to simulate waveform propagation over the digitized epicardial surfaces.

BEAT ORIGINS. We performed such simulations for each dog, with simulated beats originating from several, unique locations. Locations were chosen on the LV free wall, the RV free wall, and the remaining area (i.e. the base and apex of the ventricles, where no pacing was performed during the in vivo experiment). Origin locations within these areas were chosen pseudo-randomly, and inspected visually for a roughly uniform, equidistant distribution to prevent under- or over-representation of areas. A single beat was simulated per origin. Where not stated otherwise, the reconstructions in this Chapter used beats from 50 origins per region.

AP MODELS. To see the influence of the AP model used, we ran simulations with three different models: the neuronal FitzHugh-Nagumo model (FHN) [112], the mammalian ventricular model by Luo and Rudy (LR92) [113], and the human ventricular model by Ten-Tusscher et al. (TNNP) [114].

AP pRopagation. Simulations of AP propagation over the triangulated epicardial mesh were performed using a fast, heuristic method. The potential at each node in the mesh was modeled using a model of the single-cell AP. Currents between the nodes were introduced as $I=g \Delta V$ for every two nodes connected in the triangulation. The nodeto-node conductance $g$ was set as $g=\bar{g} / d^{2}$ where $d$ is the distance between the nodes and $\bar{g}$ is a parameter that can be manipulated to adjust the speed of propagation. In this simplified approach, we assumed conduction was the same in all directions, and did not vary from node to node. Because PBR uses spatial but not temporal characteristics of the simulated waveforms, we simply set $\bar{g}$ to any value that allowed the whole heart to activate before the first cells began to recover. These simulations were performed using Myokit, our toolkit for AP model development and simulation [115]. 
The method described above is a heuristic method, which is not guaranteed to provide the same accuracy as more advanced techniques such as finite-element based methods using the monodomain or bidomain equations (although they are based on the same physical principles). In addition, the mesh used for these simulations was rather coarse, compared to the mesh size typically used for "whole-heart" simulations. To validate the applicability of this simplified method for PBR, we performed additional highquality simulations for one of the dogs and compared the results. In these simulations, a fine-grained, regular rectangular mesh was used, representing the entire ventricular myocardium, including the left, right and septal walls. Propagation was then simulated by solving the monodomain equations using a finite-difference approximation and assuming zero-flux boundary conditions. Again, conduction in the myocardium was assumed to be homogeneous, as no knowledge of the fiber direction was available. In these detailed monodomain simulations, 29 origins per region were used. The simulations were performed using a parallelized monodomain solver [116] and the TNNP model of the AP.

\subsubsection{Creation of a Realistic basis}

For each dog and model, a set of simulated potentials $\Phi_{H}$ on the cardiac surface was obtained. These were then decomposed using singular value decomposition (SVD):

$$
\Phi_{H}=U D V^{T}
$$

Here the columns of $U$ form a spatial basis for the simulated beats, the columns of $V$ form a temporal basis, and the diagonal matrix $D$ represents the corresponding singular values. The entries in $D$ were ordered in non-increasing order, i.e. with the largest singular values first. Each singular value can be interpreted as a weight, such that a small value indicates a small contribution of the corresponding spatial and temporal patterns to describing the overall data. A truncated spatial basis $U_{k}$ was created by keeping only the first $k$ most influential elements. These capture the most prominent spatial patterns that build up the simulated potentials. Truncated bases can be beneficial as they leave fewer possibilities for ill-posed influences that could result in unrealistic solutions.

For each dog, and each AP model, we created a generic basis using simulated beats starting from all regions. In addition, region-specific bases were created using beats originating from only the LV or RV. We did not use the temporal basis or the singular values in our reconstructions. 


\subsubsection{SPARSE RECONSTRUCTION OF EPICARDIAL POTENTIALS}

Assuming that $U_{k}$ can be used as a basis for the epicardial potentials, there should be a vector $\beta$ such that

$$
\Phi_{H}(t)=U_{k} \beta(t)
$$

and our forward problem becomes

$$
\Phi_{B}(t)=A U_{k} \beta(t)
$$

By using the truncated basis $U_{k}$ instead of the full basis $U$, the reconstruction is constrained to only those elements of $U$ that contain the most relevant physiological information. Reconstruction of epicardial potentials in terms of this new basis can then be achieved by Lasso regularization [117]. This is a form of least squares approximation that minimizes the least squares error of the direct solution $\left\|A U_{k} \beta(t)-\Phi_{B}(t)\right\|_{2}^{2}$ while at the same time requiring $\|\beta\|_{1}$ to be smaller than a given parameter $\lambda(t)[117]$ :

$$
\min _{\beta(t)}\left\{\left\|A U_{k} \beta-\Phi_{B}(t)\right\|_{2}^{2}\right\} \text { subject to }\|\beta(t)\|_{1} \leq \lambda(t)
$$

This minimization can be solved for each time instant $t$ independently. The resultant $\beta(t)$ can then be plugged into equation 4.4 to obtain the heart-surface potentials at time $t$.

$L_{1}$-norm penalties have previously been shown (in a different setup) to give more accurate results than commonly used $L_{2}$-norms[118]. Constraining the $L_{1}$-norm of the parameter vector $\beta(t)$ tends to produce only a few nonzero coefficients in $\beta(t)$, leading to a sparse representation of the epicardial potentials. In other words, only the most important elements of the truncated basis will be used in the reconstruction of the epicardial potentials. As this basis consists only of well-defined spatial potential patterns, we expected that this approach would reduce the influence of ill-posedness. We used MATLAB to solve this Lasso problem, choosing $\lambda(t)$ such that the mean square error was minimized [21].

We reconstructed beats with bases generated from simulated beats originating from all regions of the heart for all three AP models $\left(\mathrm{FHN}_{\text {all }}, \mathrm{LR}_{92}\right.$ all and TNNPa all $)$. For beats paced at the $\mathrm{LV}$ or RV we compared these results to reconstructions based only on simulated beats originating from the appropriate region $\left(\mathrm{FHN}_{\text {spec }}, \mathrm{LR}_{92} 2_{\text {spec }}\right.$ and $\mathrm{TNNPa}_{\text {spec }}$ ). In one dog, reconstructions were performed using a generic and a regionspecific basis based on detailed monodomain simulations ( $\mathrm{TNNPb}_{\text {all }}$ and $\mathrm{TNNPb} \mathrm{b}_{\text {spec }}$ ). 


\subsubsection{Post-PROCESSING}

Activation and recovery times were determined from reconstructed electrograms with two different methods: a temporal-only method, and a spatiotemporal method. The temporal-only approach defines the moment of activation as the moment of steepest voltage downslope (maximum $-d \Phi_{H} / d t$ ) during the QRS complex. Recovery times were defined as the moment of maximum $d \Phi_{H} / d t$ during the $T$ wave. The spatiotemporal approach, proposed by Erem et al. [100] and also discussed in Chapter 3, takes advantage of the spatial relationship between neighboring nodes and their potentials, and could be better suited to estimate the activation time in noisy or fractionated electrograms. Erem et al. noted that not only the temporal signal (i.e. the local potential at a single node) changes quickly when an activation wavefront passes, but also the spatial gradient of potentials between neighboring nodes. Their approach to activation-time estimation selects the moment at which the change in temporal derivative coincides with the change in spatial derivative [100]. More formally, for each epicardial node, they define the activation time $\tau$ as:

$$
\tau=\min _{t}\left\|D \Phi_{H}(t)\right\|_{2} \cdot \frac{\partial \Phi_{H}(t)}{\partial t}
$$

where $\Phi_{H}(t)$ is the potential at the epicardial node under consideration at time $t, D \Phi_{H}(t)$ is the approximated spatial gradient, and $\partial \Phi_{H} / \partial t$ the approximated temporal derivative.

For recovery times, our spatiotemporal approach was to apply spatial Laplacian smoothing after temporal-only estimation. For more details on our spatiotemporal approach, see Section 7.2 of Chapter 7 .

\subsubsection{Statistical EVAluation}

For each epicardial electrode, Pearson's correlation coefficient (CC) was computed between the recorded electrogram and the reconstructed electrogram at the corresponding (closest) virtual epicardial node. Linear correlation between recorded and reconstructed activation/ recovery timings was assessed by means of Pearson's correlation coefficient R. Results were statistically compared with Wilcoxon signed-rank tests (for paired measurements) or Wilcoxon rank-sum tests (for unpaired measurements).

\subsection{Results}

Figure 4.2 shows examples of recorded and reconstructed electrograms for a sinus beat and a paced beat in one dog. Electrograms were reconstructed with PBR using different 

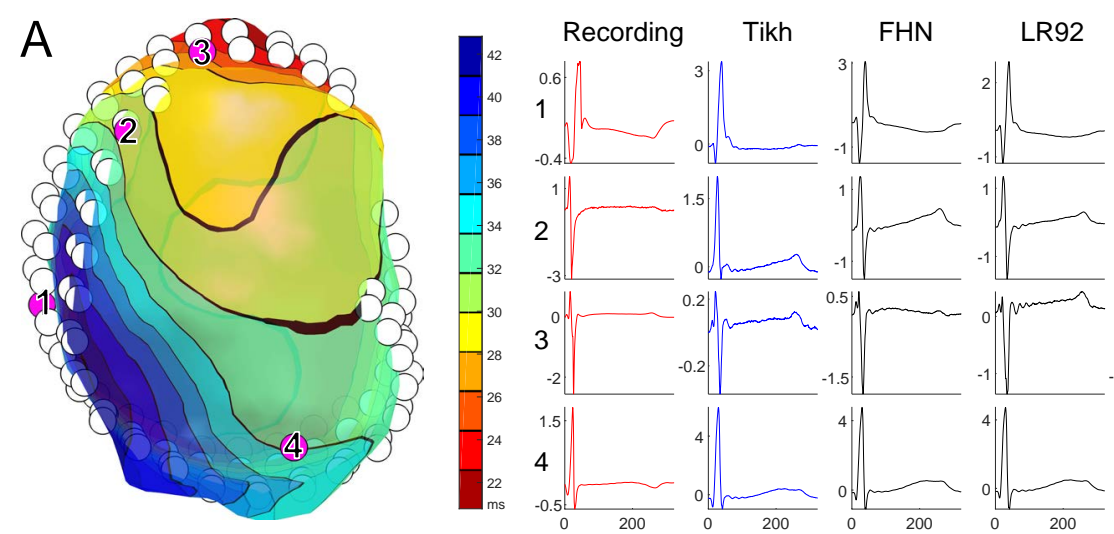

TNNPa
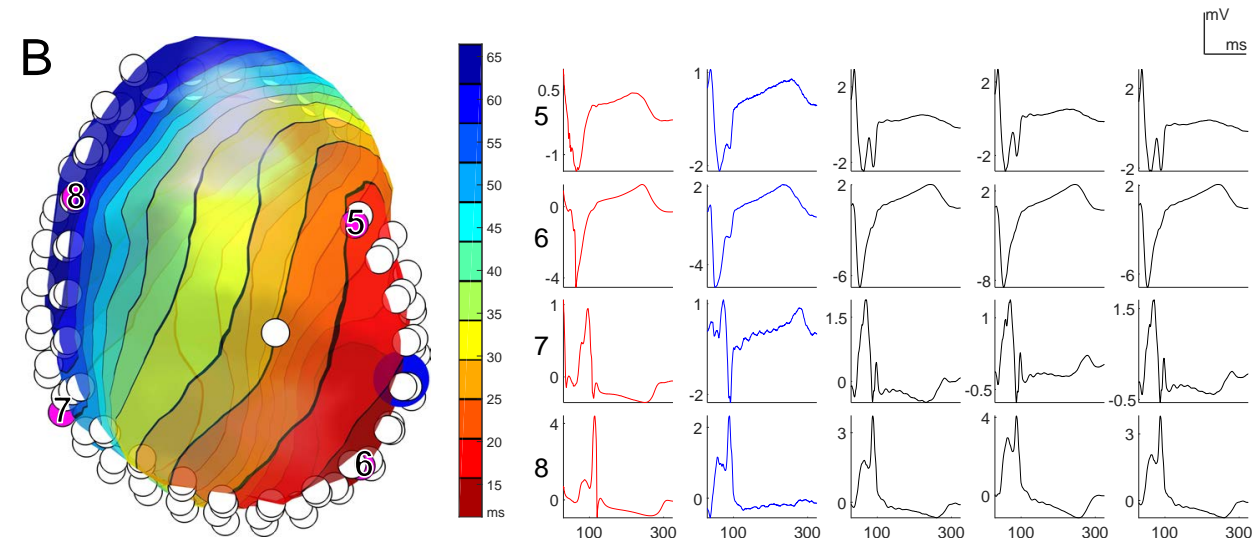

Figure 4.2: Ventricular epicardium (left, colored according to noninvasively reconstructed activation times) and recorded and reconstructed electrograms (right) during a sinus beat (panel A) and an LV paced beat (panel B, pacing location indicated by blue sphere). White circles represent the implanted epicardial electrodes. For selected electrodes (purple, numbered) the corresponding electrograms are shown: recorded (red), Tikhonov-reconstructed (blue) and electrograms reconstructed with PBR for three different basis types (FHN, LR92 or TNNPa). 
AP models, and with traditional zeroth-order Tikhonov regularization for comparison. These examples show that, regardless of the AP model, all PBR-methods were able to recover some of the electrogram characteristics that were lost with Tikhonov regularization, for example the negative deflection in electrogram 1 and 2 , and the positive deflection in electrogram 7. There were no significant changes between the different models used in PBR.

In Figure 4.3, quality of reconstruction is shown for all beats. Data were analyzed for all three dogs, for 88 beats in total (of which 67 were epicardially paced) and for 5203 epicardial electrogram pairs (recorded vs reconstructed). Results are shown for traditional regularization with Tikhonov, and for PBR using the full bases ( $\mathrm{FHN}_{\mathrm{all}}, \mathrm{LR}_{9} 2_{\text {all }}$ and $\left.\mathrm{TNNPa}_{\text {all }}\right)$, and using region-specific bases $\left(\mathrm{FHN}_{\mathrm{spec}}, \mathrm{LR}_{92} 2_{\text {spec }}\right.$ and $\left.\mathrm{TNNPa}_{\text {spec }}\right)$. Panel A of Figure 4.3 shows that the correlation between recorded and reconstructed electrograms increased significantly for 5 out of 6 PBR models. This indicates that more details were recovered by the PBR methods when compared to Tikhonov regularization, as expected from Figure 4.2.

In Panel B of Figure 4.3, activation and recovery times are shown as determined with the temporal-only method (top row) and the spatiotemporal method (bottom row). Spatiotemporal post-processing yielded more accurate timings than the temporalonly method. Activation times determined from PBR-based electrograms were no more accurate than those determined from Tikhonov-based electrograms. However, recovery times were significantly more accurate when PBR was used, especially with regionspecific bases. Combining spatiotemporal post-processing with region-specific bases gave the most accurate results.

Figure 4.3, Panel C shows that, in line with these results, localization error was improved by using spatiotemporal post-processing in all methods. Localization error did not further improve by using PBR, although using region-specific PBR did remove one outlier.

Table 4.1 shows the results for a subset of the data (one dog, 39 recorded beats), where we also used the bases generated with the whole-heart model (TNNPb). From this table, it can be seen that a simple model of the AP combined with a simplified propagation model gives the same quality PBR reconstruction as a detailed three-dimensional monodomain model.

All previous results are based on PBR reconstructions using bases that consisted of components 2-10 of $U$, that is, the first ten components of the SVD of the simulated beats, 


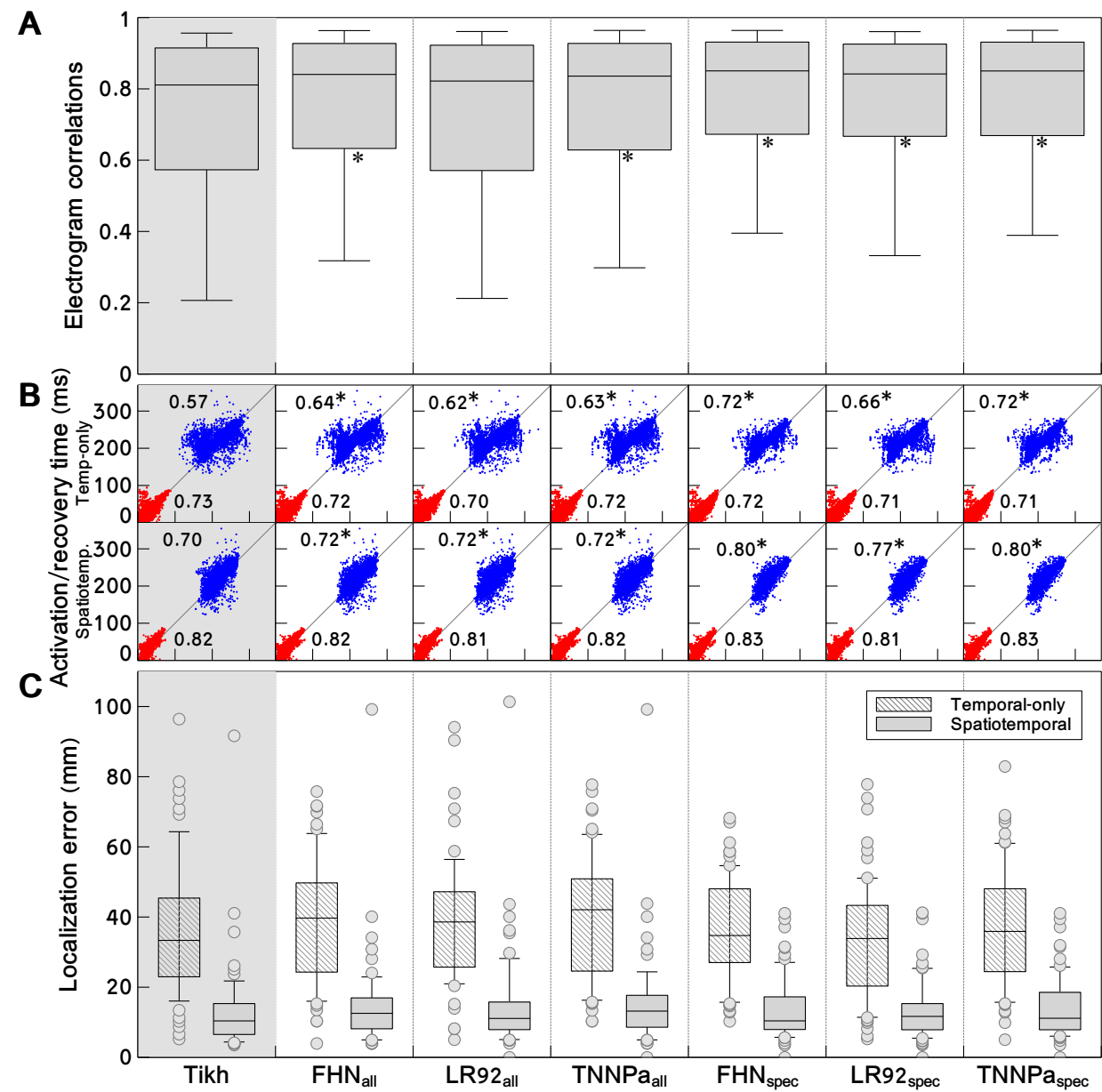

Figure 4.3: Results for the full data set. Columns show the result for the different reconstruction methods: traditional Tikhonov (Tikh) regularization, or regularization by a physiology-based method without $\left(\mathrm{FHN}_{\text {all }}, \mathrm{LR}_{2}\right.$ all and $\left.\mathrm{TNNPa} \mathrm{a}_{\text {all }}\right)$ and with $\left(\mathrm{FHN}_{\mathrm{spec}}\right.$, $\mathrm{LR92}_{\text {spec }}$ and $\mathrm{TNNPa}_{\text {spec }}$ ) region-specific bases. Panel A: box plots of correlation coefficients between recorded and reconstructed electrograms. Box spans the interquartile range (IQR), i.e., the $25-75 \%$ range; median indicated by horizontal line; whiskers at $9-91 \%$ range. Overall, PBR improves reconstruction quality, especially using region-specific bases. Panel B: Activation times (red) and recovery times (blue) as reconstructed (horizontal axes) vs. recorded (vertical axes). Top row shows these timings as directly determined from the electrograms, i.e. with temporal-only criteria. Bottom row shows timings as determined with a spatiotemporal method. Recovery times, especially, are improved by PBR. Additional improvement is achieved when spatiotemporal post-processing is used. Panel C: Localization error between detected and known origins of pacing, as determined with temporal-only methods (hatched box plots) and with spatiotemporal methods (gray box plots). A combined use of spatiotemporal post-processing and PBR gives most accurate results. An asterisk $\left({ }^{*}\right)$ indicates significant improvement compared to Tikhonov results. 


\begin{tabular}{|c|c|c|c|c|}
\hline Model & $\mathrm{CC}_{\mathrm{EGM}}$ & $\mathrm{R}_{\mathrm{act}}$ & $\mathrm{R}_{\mathrm{rec}}$ & $\mathrm{LE}(\mathrm{mm})$ \\
\hline Tikh & 0.81 & 0.8 & 0.58 & 39 \\
\hline$\overline{\mathrm{F}} \overline{\mathrm{H}} \overline{\mathrm{N}}_{\mathrm{all}}$ & $0.85^{*}$ & 0.77 & $0.62^{*}$ & $44^{*}$ \\
\hline LR92 all & $0.83^{*}$ & 0.77 & 0.59 & 42 \\
\hline $\mathrm{TNNPa}_{\text {all }}$ & $0.84^{*}$ & 0.77 & $0.60^{*}$ & $47^{*}$ \\
\hline TNNPb $b_{\text {all }}$ & $0.84^{*}$ & 0.77 & $0.62^{*}$ & 45 \\
\hline$\overline{\mathrm{F}} \overline{\mathrm{H}} \overline{\mathrm{N}}_{\mathrm{spec}}-$ & $0.87^{*}$ & 0.78 & $0.79^{*}$ & $3 \overline{9}$ \\
\hline $\mathrm{LR}_{92} 2_{\text {spec }}$ & $0.87^{*}$ & 0.78 & $0.69^{*}$ & 39 \\
\hline $\mathrm{TNNPa}_{\text {spec }}$ & $0.87^{*}$ & 0.78 & $0.79^{*}$ & 39 \\
\hline$T_{N N P b_{\text {spec }}}$ & $0.87^{*}$ & 0.78 & $0.76^{*}$ & 40 \\
\hline
\end{tabular}

Table 4.1: Effect of more complex AP model. Median accuracy metrics in one dog (39 recorded beats), investigating the added value of a detailed whole heart model (TNNPb). *, statistically significant difference with Tikhonov results. Activation and recovery times (and beat origins) determined from temporal-only criteria. LE: localization error, i.e., the distance between the known pacing location and reconstructed location of earliest activation.

not including the first component. The first component is a constant negative pattern, reflecting the $-80 \mathrm{mV}$ offset of the simulated APs. As we reconstruct electrograms (with a zero average), not APs, this offset component does not have a role for our purpose. Components 2-10 for a FHN-based set of simulated beats are shown in Panel $\mathrm{C}_{1}$ of Figure 4.1. In Figure 4.4, the basis components are shown for all AP models and beat origins in one dog. Reconstruction accuracy with different components of the SVD as basis is shown in Table 4.2 for components $2-5,2-10,2-15$ and $2-25$ for the full data set. For most metrics, using components $2-10$ gave the best result.

Not only the basis size $k$ is important, but also the number of morphologicallydistinct beats that was used to create that basis. In Figure 4.5, the dependency of PBRaccuracy on the number of simulated unique beats is explored in a subset of the data (one dog, 39 recorded beats) with the $\mathrm{FHN}_{\text {all }}$ basis. Both correlation between recorded and reconstructed electrograms, and the accuracy of activation and recovery times are maxi-

Figure 4.4 (following page): Bases created with PBR for different AP models and simulated beat origins, in one dog. Each row shows bases elements $2-10$ as created with a specific AP models (FHN, LR92, TNNPa or TNNPb) and a specific region of simulated origins (full epicardial surface ['all'], only the LV ['left'], or only the RV ['right']). The elements are shown in reducing order (left-to-right) of contribution to the simulated potentials. Regardless of AP method and beat origins, the first basis elements capture simple patterns and later elements capture more complex patterns. There is a clear difference between basis elements of different beat origins (all vs left vs right), but less difference between basis elements of different AP models. 


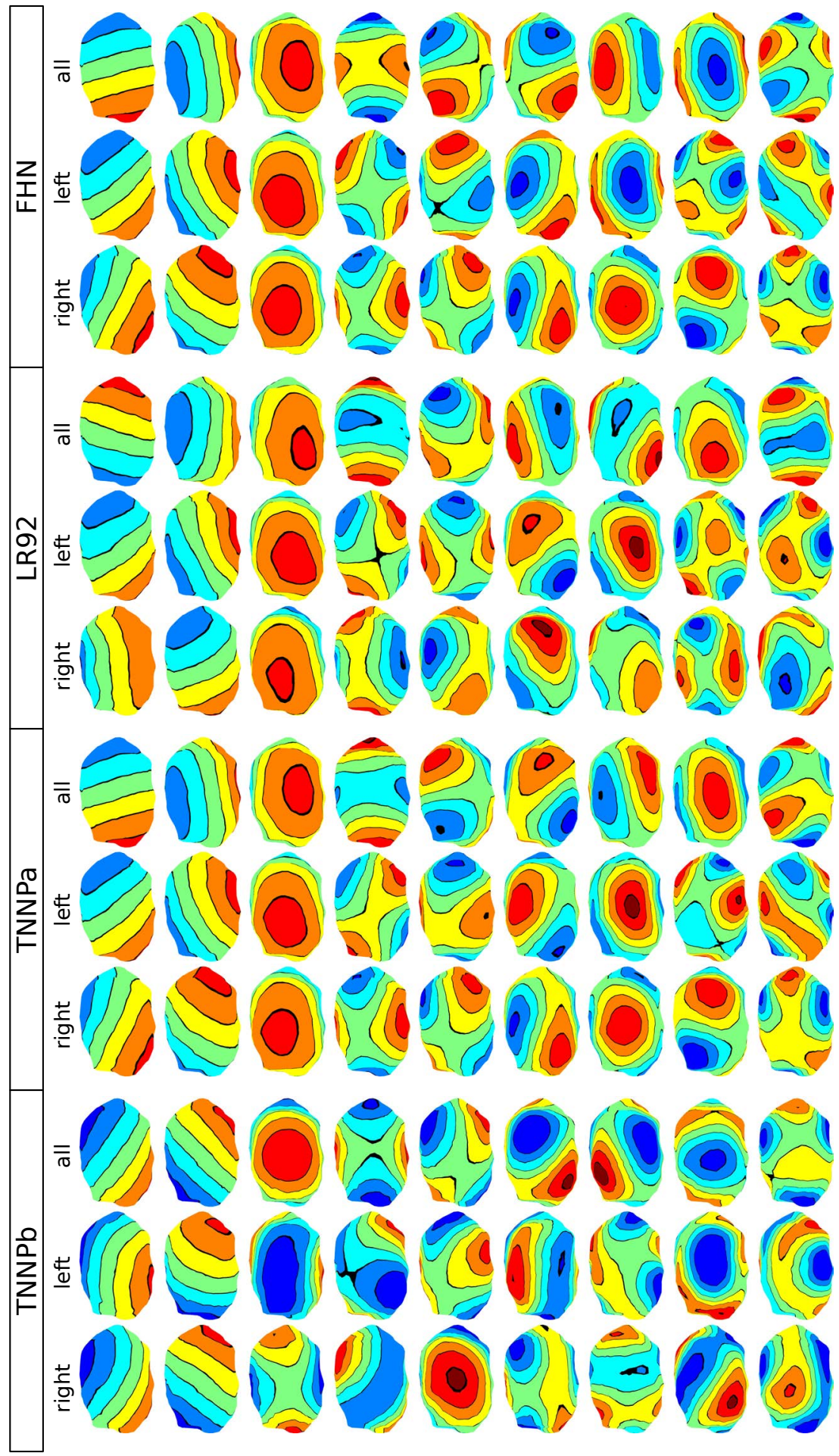




\begin{tabular}{|c|c|c|c|c|c|c|c|}
\hline \multicolumn{8}{|c|}{ Correlation coefficients for electrograms (CC) } \\
\hline 5 & 0.81 & $0.80^{*}$ & $0.79^{*}$ & $0.80^{*}$ & $0.83^{*}$ & $0.83^{*}$ & $0.83^{*}$ \\
\hline 10 & $”$ & $0.84^{*}$ & 0.82 & $0.84^{*}$ & $0.85^{*}$ & $0.84^{*}$ & $0.85^{*}$ \\
\hline 15 & & 0.81 & 0.81 & 0.81 & 0.82 & 0.81 & $0.82^{*}$ \\
\hline 25 & $”$ & $0.74^{*}$ & $0.73^{*}$ & $0.73^{*}$ & $0.72^{*}$ & $0.74^{*}$ & $0.71^{*}$ \\
\hline \multicolumn{8}{|c|}{ Activation time correlation $(\mathrm{R})$} \\
\hline 5 & 0.73 & 0.68 & 0.66 & 0.68 & 0.65 & 0.65 & 0.64 \\
\hline 10 & & 0.72 & 0.70 & 0.72 & 0.72 & 0.71 & 0.71 \\
\hline 15 & & 0.72 & 0.71 & 0.72 & 0.70 & 0.67 & 0.69 \\
\hline 25 & $"$ & 0.62 & 0.57 & 0.62 & 0.51 & 0.58 & 0.49 \\
\hline \multicolumn{8}{|c|}{ Recovery time correlation $(\mathrm{R})$} \\
\hline 5 & 0.57 & $0.62^{*}$ & 0.60 & $0.62^{*}$ & $0.69^{*}$ & $0.67^{*}$ & $0.69^{*}$ \\
\hline 10 & & $0.64^{*}$ & $0.62^{*}$ & $0.63^{*}$ & $0.72^{*}$ & $0.66^{*}$ & $0.72^{*}$ \\
\hline 15 & & 0.58 & 0.57 & 0.58 & $0.61^{*}$ & $0.62^{*}$ & $0.61^{*}$ \\
\hline 25 & $"$ & $\begin{array}{l}1 \\
0.41 \\
\end{array}$ & 0.39 & 0.42 & 0.37 & 0.36 & 0.35 \\
\hline \multicolumn{8}{|c|}{ Localization error (mm) } \\
\hline 5 & 33 & $48^{*}$ & $45^{*}$ & $48^{*}$ & $46^{*}$ & $41^{*}$ & $45^{*}$ \\
\hline 10 & & 40 & 39 & $42^{*}$ & 35 & 34 & 36 \\
\hline 15 & $"$ & 34 & 30 & 33 & 35 & 36 & 34 \\
\hline 25 & $"$ & I 37 & $45^{*}$ & 37 & $41^{*}$ & 38 & $36^{*}$ \\
\hline
\end{tabular}

Table 4.2: Dependency on basis size. Median accuracy metrics for the full data set for different number of components of the realistic basis (basis size $k$ ). ${ }^{*}$, statistically significant difference with Tikhonov results. Activation and recovery times (and beat origins) determined from temporal-only criteria.

mal when data from 6 or more simulated beats is included in the basis $U_{k}$.

\subsection{Discussion}

We have shown in an in vivo experiment that PBR increases the accuracy of reconstruction of electrograms and recovery times, compared to traditional Tikhonov regularization, and was able to recover electrogram characteristics that were lost with Tikhonov regularization. This indicates that PBR yields more information in the reconstructed electrograms than traditional methods.

\subsubsection{RECOVERY AND ACTIVATION TIMES}

Recovery times were determined more accurately with PBR than with Tikhonov regularization, and therefore profited less from spatiotemporal post-processing. However, a 


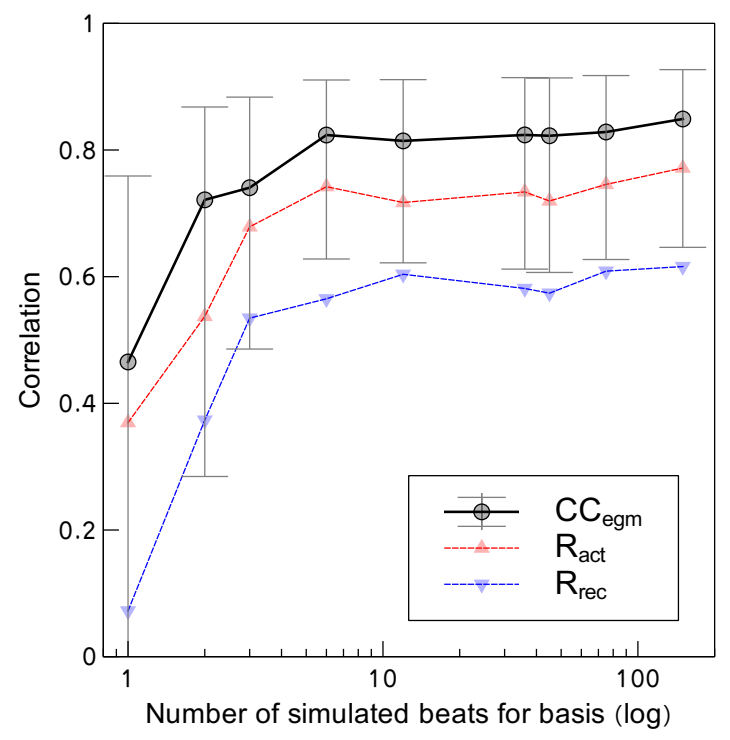

Figure 4.5: Dependency of the accuracy of PBR on the number of (unique) simulated beats that are used for the basis. Results for one dog (39 recorded beats), reconstructed with $\mathrm{AP}$ model $\mathrm{FHN}_{\text {all }}$. Correlation coefficients between recorded and reconstructed electrograms $\left(\mathrm{CC}_{\text {egm }}\right)$ are maximal and stable when approximately 6 or more beats are simulated. Similarly, activation times $\left(R_{\text {act }}\right)$ and recovery times $\left(R_{\text {rec }}\right)$ are reconstructed with highest accuracy for 6 or more simulated beats.

combination of PBR with spatiotemporal post-processing yielded the highest accuracy for recovery times. PBR did not improve accuracy of activation times, which were already determined with reasonable accuracy. Abnormalities in recovery patterns can be an important substrate for cardiac arrhythmia and they are often difficult to diagnose from the 12-lead ECG due to its limited spatial resolution. Noninvasive imaging of recovery abnormalities could therefore greatly benefit risk assessment and further understanding of their role in arrhythmia.

\subsubsection{BEAT ORIGIN LOCALIZATION}

Beat origin localization is performed by finding the location with the earliest activation time. Since the accuracy of the estimated activation times did not improve significantly with PBR, beat origin locations were not significantly improved. However, some outliers were removed when region-specific bases were used. This is relevant for clinical practice, where the origin of a ventricular ectopic beat is often a target for invasive ablation therapy. More accurate localization of ectopic beats could help guide therapy, thereby reducing procedural time and improving success rates. 


\subsubsection{TUNING THE BASES IMPROVES RESULTS}

One interesting feature of $\mathrm{PBR}$ is that its bases can be tuned to a specific clinical question. From a 12-lead ECG, the LV or RV origin of an ectopic beat can often be determined without issues. In this study we have shown that, using a basis specific to the LV or RV, a higher accuracy of electrogram reconstruction is achieved, along with a reduction of origin-localization outliers.

Interestingly, the AP model used did not affect reconstruction quality, and a simplified model of AP propagation was found to perform as well as a fine-grained monodomain simulation. This may be explained by the fact that the truncated basis elements reflect the spatial potential patterns, mainly reflecting the order of activation. Consequently, as demonstrated in Figure 4.4, the basis patterns obtained with SVD using different models are very similar, unless spatial differences exist such as limited regions of beat origins.

This means that PBR leaves temporal aspects largely unconstrained and it is unlikely that incorporating temporal patient-specific characteristics will benefit the results. However, including spatial information such as infarcted regions could directly influence reconstruction quality: these characteristics will have a large impact on simulated beat propagation and will therefore be incorporated in the truncated spatial basis. Similarly, incorporating spatio-temporal information such as abnormal regional changes to the AP may lead to improved reconstructions. These results suggest that even simpler methods for simulating propagating waves, for example using the eikonal equations, may be applicable to PBR in its basic form. However, such methods do not offer as clear a road to integrating patient-specific spatio-temporal characteristics such as local changes in the ionic balances. As the dogs used in this study had normal hearts, we were not able to investigate these hypotheses.

\subsubsection{Parameter Dependency}

PBR is able to reconstruct electrophysiologically relevant patterns using a limited number of basis elements (typically 9) generated from only a few simulated beats ( 6 or more). This is possible, because a linear combination of these basis elements still spans a large enough solution space, even when this truncated basis only consists of the first, simple basis patterns. The Lasso method allows any linear combination, but gives preference to sparse solutions, thereby reducing the influence of ill-posedness. The combination of a truncated realistic basis with Lasso optimization will result in solutions based on a small number of electrophysiologically relevant 'building blocks'. 
An open question is how to automatically determine the number of elements needed in the truncated basis. With too few elements, the basis will not span the full range of possible potential patterns and the method will be unable to reconstruct all physiologically relevant cases. If too many elements are used, the ill-posedness of the inverse problem will dominate and allow noise-like influence of the 'less important' basis elements to obscure the real solution. In this study, we found that the optimal basis size was close to 10. For clinical applications, where no invasively measured potentials are available, methods are needed that automatically determine the optimal basis size. These should aim to find a balance where ill-posedness is reduced without overly constraining the solution space.

\subsubsection{OTHER APPROACHES}

In another study [119], a method was presented to reconstruct the heart-surface potentials based on a-priori knowledge about their spatial covariance. This knowledge is usually not available, and this method therefore cannot be applied in practice. However, with methods similar to those presented in the present Chapter, knowledge about the spatial covariance could be simulated, making it worthwhile to re-investigate this approach.

In another recent study [110], heart-surface potentials were reconstructed with the help of a simulation based on the bidomain equations, similar to the TNNPb monodomain simulations performed in this Chapter. We have shown that, in our implementation, such a detailed cellular implementation does not contribute to the accuracy of reconstructions.

We have presented a method to include electrophysiological data in the potentialbased formulation of the inverse problem. In the wavefront-based formulation of the inverse problem, (cellular) electrophysiology models have always been part of the reconstruction process, e.g. by including a model of the expected transmembrane potentials $[52],[120]$.

\subsection{Conclusion}

We have introduced and validated PBR, a novel method to noninvasively reconstruct epicardial potentials from body-surface potentials. By incorporating simulated electrophysiological input in the regularization of the inverse problem of electrocardiography, more information is recovered in the reconstructed epicardial electrograms. Reconstruction of recovery time, in particular, is improved with this method. While we found that the level of temporal detail in the simulations did not affect the results, the inclusion of spatial characteristics (i.e., suspected LV or RV origin) did improve accuracy. Nonin- 
vasive imaging of recovery abnormalities with PBR can greatly benefit risk assessment and improve understanding of their role in arrhythmias. This may help to answer clinical questions with improved accuracy.

\section{ACKNOWLEDGEMENTS}

The authors would like to thank Roel L.H.M.G. Spätjens for help with the figures, and Joël M.H. Karel and Pietro Bonizzi for technical discussions. Furthermore, the authors would like to thank Dana Brooks, Jaume Coll-Font, (Northeastern University, Boston, MA, USA), and Burak Erem (Harvard Medical School and Boston Children's Hospital, MA, USA), for kindly sharing their algorithm for spatiotemporal activation-time approximation.

\section{CONFLICTS OF INTEREST}

Conflict of interest: none declared 


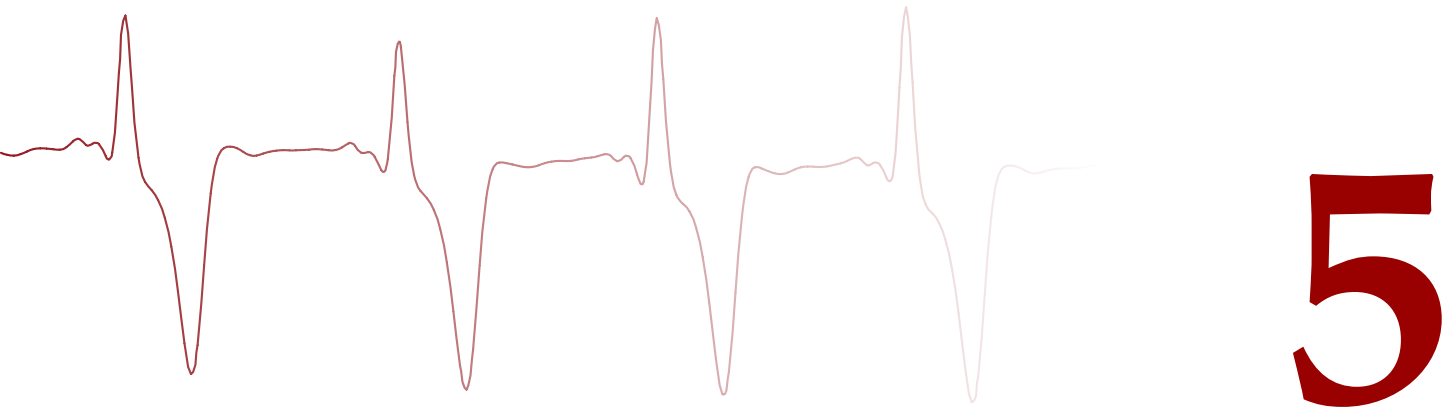

Electrocardiographic imaging: regularization with spatiotemporal sparsity in the wavelet domain

\section{Contents}

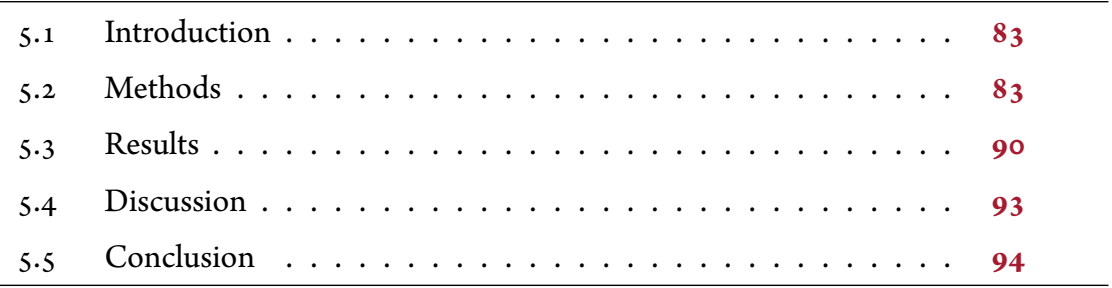




\section{Abstract}

Objective: To investigate a novel sparsity-based regularization in the wavelet domain of the inverse problem of electrocardiography.

Methods: In three normal, anesthetized dogs, electrodes were implanted around the epicardium and body-surface electrodes were attached to the torso. Potential recordings were obtained simultaneously on the body-surface and on the epicardium. A CT scan was used to digitize a homogeneous geometry which consisted of the body-surface electrodes and the epicardial surface. Beats were recorded during normal sinus rhythm and during epicardial pacing. A novel elasticnet based method was introduced to regularize the inverse problem of electrocardiography. The method simultaneously pursues a sparse wavelet representation in time-frequency and exploits correlations in space. Performance was assessed in terms of quality of reconstructed epicardial potentials, estimated activation and recovery time, and estimated locations of pacing, and compared with performance of Tikhonov zeroth-order regularization.

Results: Epicardial potentials were noninvasively reconstructed with higher accuracy than with Tikhonov zeroth-order regularization $(p<0.05)$, and recovery times were improved $(p<0.05)$. No significant improvement was found in terms of activation times and localization of origin of pacing.

Conclusion: Multi-task elastic-net based regularization of the inverse problem of electrocardiography in the wavelet domain improved both quality of electrogram reconstruction and recovery time estimation.

Significance: Abnormalities in recovery are an important substrate for cardiac arrhythmias and sudden cardiac death, and more accurate estimates of epicardial electrograms and recovery times could directly impact clinical interpretation.

Adapted from: MJM Cluitmans*, JMH Karel*, P Bonizzi, PGA Volders, RL Westra, RLM Peeters. Electrocardiographic imaging: regularization with spatiotemporal sparsity in the wavelet domain. Submitted.

* The first two authors contributed equally to this work. 


\subsection{INTRODUCTION}

As discussed in the previous chapter, the reconstruction quality of ECGI is influenced by ill-posedness, reducing the accuracy of the reconstructed electrical heart activity. To cope with this problem, regularization is applied, i.e., additional knowledge is incorporated, in the form of constraints on the possible solutions, in order to arrive at more realistic results [36], [108]. For a review of common regularization methods, see [82], [98]. One way to overcome the influence of ill-posedness is to work with the data in a different domain. For example, in Chapter 4 we have shown that accuracy is improved when heart-surface potentials are determined as a function of physiologically-realistic potential patterns obtained from numerical models that function as 'building blocks'. By using relevant building blocks only, a sparse representation was acquired, which leaves only little room for ill-posedness and could therefore significantly improve reconstruction accuracy. In the present Chapter, we propose to use a different sparse representation, in terms of wavelets. By using an appropriate wavelet basis, only few wavelet expansion coefficients are necessary to describe electrograms at the heart surface.

Additionally, our previous regularization method works (as many other commonly used methods [82], [98]) on spatiotemporally decoupled versions of the inverse problem, where the regularization applies only on the spatial distribution of potentials (at a certain time point) or on the temporal behaviour of potentials (at a certain spatial location). In contrast, the method proposed in the current Chapter achieves regularization over space and time simultaneously.

The purpose of this Chapter was to investigate whether such a spatiotemporal regularization of a sparse representation of the electrical potentials at the heart surface in the wavelet domain could improve accuracy. We evaluated this in canine experiments for which simultaneous body-surface and invasive epicardial recordings were obtained.

\subsection{Methods}

\subsubsection{THE INVERSE PROBLEM OF ELECTROCARDIOGRAPHY}

The potential-based formulation of the forward and inverse problems of electrocardiography is based on the assumption that there is a direct and instantaneous linear relationship between potentials at a closed surface surrounding the heart, and the potentials at the body surface [82], as we have also discussed in the previous Chapters. The closed surface surrounding the heart is usually taken to be the epicardium, i.e., the outer heart 
surface. The forward model describes the way that body-surface potentials are induced by heart-surface potentials. It is commonly defined as:

$$
\Phi_{B}=A \Phi_{H}
$$

where $\Phi_{B}$ is an $r \times m$ matrix of potentials at $r$ body-surface nodes at $m$ moments in time, and $\Phi_{H}$ a $q \times m$ matrix of potentials at $q$ heart-surface nodes at the same $m$ time instants. The $r \times q$ transfer matrix $A$ depends on the geometry (the node locations, both on the torso and on the heart) and the medium (the conductivity properties of the body tissues). The model expresses an instantaneous, linear, and time-invariant relationship, motivated by the quasi-static nature of electromagnetic fields in the torso and the superposition principle. It neglects the influences of tissue capacitance and any sources of electrical potentials other than the heart, as well as the effects of relative motion of the nodes (e.g., due to a contracting beating heart and breathing). It also assumes that the $q$ heart-surface nodes are sufficiently densely generated to well represent all electrical potential on the heart. The matrix $A$ is typically estimated based on a computed tomography (CT) scan of the patient's torso with body-surface electrodes attached.

The objective of the inverse problem of electrocardiography is to find $\Phi_{H}$ so as to best explain a recorded instance of $\Phi_{B}$ taking into account the estimated transfer matrix A:

$$
\min _{\Phi_{H}}\left\|A \Phi_{H}-\Phi_{B}\right\|_{2}^{2} .
$$

Here the notation $\|\cdot\|_{2}$ is used to indicate the entrywise 2-norm. Throughout this Chapter we shall employ it for vectors and matrices alike; in the former case it expresses the usual Euclidean norm, in the latter case the matrix Frobenius norm. The choice of this norm is motivated by the time-invariance of the forward linear model, the identical technical specifications of the electrodes, and the assumed independence and (Gaussian) whiteness of the measurement errors.

Though equation (5.2) is of the well-known linear least squares type, in this case there are a number of complicating factors which make it ill-posed and hard to solve accurately:

Near dependencies in the rows of $A$ : In the situation that the nodes on the body surface are densely placed, it is to be expected that the value at a given node can often be estimated well by a linear combination of the values at neighboring nodes. As a result, many of the nonzero singular values of $A$ will be small in size. Their reciprocals are used in the reconstruction of $\Phi_{H}$, making the computation unstable. 
Underdeterminacy: To have sufficient resolution at the heart surface, the number of heart-surface nodes $q$ is generally much larger than the number of electrodes $r$. For each moment in time this leads to non-unique solutions: the space of all minimizers of equation (5.2) has many degrees of freedom. The aforementioned near dependencies worsen this situation.

This ill-posedness of the inverse problem tends to cause disproportionately large and rather unpredictable changes in the computed heart-surface potentials, when only slight variations in the body-surface potentials occur, e.g., due to noise or measurement errors. Other contributing factors to this inherent unreliability of the solution, are that the forward model is only approximate, while the matrix $A$ can only be estimated with limited accuracy too, due to necessary simplifications with respect to non-linearities, torso inhomogeneities, movement of heart and torso, changes in conductivity, and so on.

Therefore, to obtain a stable solution to the inverse problem additional constraints on the feasible solutions are needed, either implicit or explicit. For example, the well-known Tikhonov regularization method [93] does this implicitly. It obtains a stable solution by penalizing the amplitude of the reconstructed cardiac potentials (or derivatives thereof) and solving the resulting modified linear least squares problem:

$$
\min _{\Phi_{H}}\left\{\left\|A \Phi_{H}-\Phi_{B}\right\|_{2}^{2}+\lambda\left\|R \Phi_{H}\right\|_{2}^{2}\right\} .
$$

Here $R$ is the regularization operator. It equals the identity matrix for zeroth order, the gradient operator for first order, or the Laplacian for second order Tikhonov regularization. The regularization parameter $\lambda$ balances the least squares error of the fit with the amount of regularization. It can be selected with methods such as the L-curve approach [92].

5.2.2 Elastic-Net BASED RECONSTRUCTION OF EPICARDIAL POTENTIALS With WAVELET-DOMAIN REGULARIZATION

Another approach to regularization is to reduce parameter redundancy by representing $\Phi_{H}$ sparsely. One may do this explicitly, by preselecting a limited number of 'building blocks' to construct $\Phi_{H}$ with, as in [109], [121]. An implicit, more flexible way to obtain sparsity, is by minimizing the least squares error criterion under an $\ell_{0}$-norm constraint. However, the $\ell_{\mathrm{o}}$-norm constraint yields a nonconvex optimization problem, which is computationally difficult to solve. Therefore, the $\ell_{1}$-norm is widely used as an alternative that also promotes sparsity [122], for example in the lasso method [117], or in the more general elastic-net approach [123], [124]. 
If each of the $m$ columns of the heart-surface potential matrix $\Phi_{H}$ were sparse, then the elastic-net approach would give rise to $m$ decoupled elastic net problems which could be solved independently:

$$
\min _{\Phi_{H}^{(k)}}\left\{\left\|A \Phi_{H}^{(k)}-\Phi_{B}^{(k)}\right\|_{2}^{2}+\lambda\left[(1-a) \frac{1}{2}\left\|\Phi_{H}^{(k)}\right\|_{2}^{2}+a\left\|\Phi_{H}^{(k)}\right\|_{1}\right]\right\}, \quad k=1, \ldots, m,
$$

where the superscipt ${ }^{(k)}$ denotes the $k$-th column of a matrix, corresponding to the $k$-th time point. In this case the problem is decoupled over time and sparsity is sought over space, per time instant. The constant $\alpha$ is the elastic-net mixing parameter, balancing between a lasso approach $(\alpha=1)$ and zeroth order Tikhonov regularization (ridge regression, $a=0$ ). The factor $\frac{1}{2}$ appears for consistency with [124] and the software glmnet [125].

However, there is no physiological reason for $\Phi_{H}$ to be sparse over space. Instead, it makes more sense to pursue sparsity of a suitably chosen representation of $\Phi_{H}$. Here we propose that such a representation is most easily achieved by applying an orthogonal wavelet transform over time only. Even though a wavelet transform can also be applied spatially, the fact that the heart-surface is curved and the nodes are all lumped into a single vector makes it difficult to handle it properly, leaving us wanting to avoid the spatial dimension.

The temporal evolution of potentials on a single node is known as an electrogram. It is well understood and can commonly be sparsely represented with the orthogonal Daubechies-2 wavelet [126]. If desired, one may also design a more dedicated orthogonal wavelet using the approach of [127].

For an orthogonal wavelet, the set of the wavelet (and approximation) coefficients up to a chosen decomposition level can be obtained from the time signal at a node, by applying a corresponding orthogonal matrix $W$. Then the matrix $P_{H}$ of wavelet coefficients of all the electrograms in the rows of $\Phi_{H}$ is given by:

$$
P_{H}=\Phi_{H} W
$$

Multiplication by an orthogonal matrix does not change the 2-norm, and it is natural to require the matrix $P_{H}$ to be sparse. One may then proceed to address and solve the 
following set of $m$ decoupled elastic net problems:

$$
\min _{P_{H}^{(k)}}\left\{\left\|A P_{H}^{(k)}-P_{B}^{(k)}\right\|_{2}^{2}+\lambda\left[(1-a) \frac{1}{2}\left\|P_{H}^{(k)}\right\|_{2}^{2}+a\left\|P_{H}^{(k)}\right\|_{1}\right]\right\}, \quad k=1, \ldots, m,
$$

where $P_{B}=\Phi_{B} W$ denotes the matrix of body-surface wavelet coefficients using the same orthogonal wavelet.

Each $P_{H}^{(k)}$ denotes a vector of wavelet coefficients of index $k$, collected over all nodes. The index $k$ corresponds to a specific localized time and scale pair. This means that by solving for each $P_{H}^{(k)}$ independently, the problem gets decoupled over the wavelet coefficients and the natural relationship between the wavelet coefficients within a scale due to wave propagation is ignored.

To take into account that there will be correlations over time and across wavelet scales, it is proposed that one finally combines the $m$ elastic net problems into a single multi-task lasso problem [128], [129]. Then joint sparse estimation of the coefficients can be achieved by including a penalty term consisting of the $\ell_{1}$-norm of the root energies per wavelet coefficient over all the grid points:

$$
\min _{P_{H}}\left\{\left\|A P_{H}-P_{B}\right\|_{2}^{2}+\lambda\left[(1-\alpha) \frac{1}{2}\left\|P_{H}\right\|_{2}^{2}+\alpha \sum_{k=1}^{m}\left\|P_{H}^{(k)}\right\|_{2}\right]\right\}
$$

This promotes sparsity over all the wavelet coefficients simultaneously, over timefrequency and space. The last penalty term of equation $(5.7)$ is a mixed $\ell_{2} / \ell_{1}$-norm, i.e., the $\ell_{1}$-norm of the vector of columnwise $\ell_{2}$-norms of $P_{H}$. For computational purposes, the multi-task lasso problem can be formulated in turn as a group-lasso problem [124] for which standard packages exist such as glmnet [125].

The proposed approach expressed in equation (5.7) exploits the sparsity of the electrograms in the wavelet domain, is able to exploit the coherence between wavelet coefficients and takes into account zeroth order Tikhonov penalties. From the resulting wavelet coefficient matrix $P_{H}$, the desired heart-surface potentials are eventually obtained by $\Phi_{H}=P_{H} W^{T}$.

\subsubsection{IN VIVO RECORDINGS}

In vivo data were acquired in the canine experiment discussed in Chapter 3 . In short, in three normal, anesthetized dogs, 99 electrodes were implanted around the epicardium via a thoracotomy and 192 body-surface electrodes were attached to the torso after chest 
closure. Potential recordings were obtained simultaneously on the body-surface and on the epicardium. A CT scan was performed and used to digitize a homogeneous geometry which consisted of the body-surface electrodes and the epicardial surface. The transfer matrix, relating the electrical activity at the cardiac surface to the body surface, was computed with methods available from the SCIrun software repository [19]. Beats were recorded during normal sinus rhythm and during epicardial pacing. Segmentation of the surfaces from the CT scan was performed with $\mathrm{Seg}_{3} \mathrm{D}$ [18]. The ventricular epicardium was digitized as a triangulated mesh. This procedure requires a manual segmentation, causing variability in the number of nodes. On average 1693 nodes were obtained, which then acted as the cardiac surface.

Epicardial potentials were reconstructed with the wavelet-based regularization method described in the previous section. For this, we used the Daubechies-2 (i.e., filter length 4) wavelet. We used 3 levels of wavelet decomposition, which provided results that were as accurate as when more levels were used, but computationally more efficient. We choose the stationary-wavelet approach over the discrete-wavelet approach because of its time-invariant properties. The optimization of equation (5.7) was performed with the glmnet package in Matlab [21], [125].

\subsubsection{POST-PROCESSING}

From reconstructed electrograms, activation and recovery times were determined with a spatiotemporal method. This approach, proposed by Erem et al [100], takes advantage of the spatial relationship between neighboring nodes and their potentials, and could be better suited to estimate the activation time in noisy or fractionated electrograms then temporal-only criteria. The authors noted that not only the temporal signal (local potential at a single node) changes quickly when an activation wavefront passes, but also the spatial gradient of potentials between neighboring nodes. Their approach to activation-time estimation selects the moment in which the negative temporal derivative and change in spatial derivative coincide [100]. More formally, for each epicardial node, they define the activation time $\tau$ as:

$$
\tau=\min _{t}\left\{\|D \Phi(t)\|_{2} \cdot \frac{\partial \Phi(t)}{\partial t}\right\}
$$

where $\Phi(t)$ is the potential at the epicardial node under consideration at time $t, D \Phi(t)$ is the approximated spatial gradient, and $\partial \Phi / \partial t$ the approximated temporal derivative. For recovery times, our spatiotemporal approach was to apply spatial Laplacian smoothing after temporal-only estimation [130]. 


\subsubsection{ANALYSis OF PERFORMANCE}

Given the multi-task elastic-net model in equation (5.7), a grid-search was carried out to identify an optimal point in the two-dimensional parameter space given by the regularization parameter $\lambda$ and the elastic-net mixing parameter $\alpha$. Optimality was defined in terms of accuracy of reconstruction by looking at the median correlation coefficient between recorded electrogram and the reconstructed electrogram at the corresponding (closest) virtual epicardial node, with the former representing the ground truth in this study. Eight beats from one dog (out of eighty-nine beats from all three dogs) were used for this optimality analysis. The choice of eight beats was constrained by the computational effort of this analysis. For completeness, we also looked at the data mismatch, i.e. the mismatch between the recorded body-surface potentials and body-surface potentials provided by the forward solution based on reconstructed epicardial potentials as in equation (5.1). The data mismatch is usually applied as criterion when no ground truth is available.

After identifying optimal values for $\lambda$ and $\alpha$, we used the corresponding model to assess performance of the proposed approach. Performance was assessed in terms of accuracy of:

Reconstructed epicardial potentials. For each epicardial electrode, Pearson's correlation coefficient (CC) was computed between the recorded electrogram and the reconstructed electrogram at the corresponding (closest) virtual epicardial node;

Estimated activation and recovery time. Linear correlation between recorded and reconstructed activation/recovery timings was assessed by means of Pearson's correlation coefficient;

Estimated locations of pacing. Localization error (LE) was defined as the Euclidean distance between the reconstructed location of earliest activation and the known pacing location.

Results were statistically compared with Wilcoxon signed-rank tests (for paired measurements) or Wilcoxon rank-sum tests (for unpaired measurements). Performance of the proposed approach was compared with Tikhonov zeroth-order regularization, one of the most commonly used regularization approaches in the inverse problem of electrocardiography. 

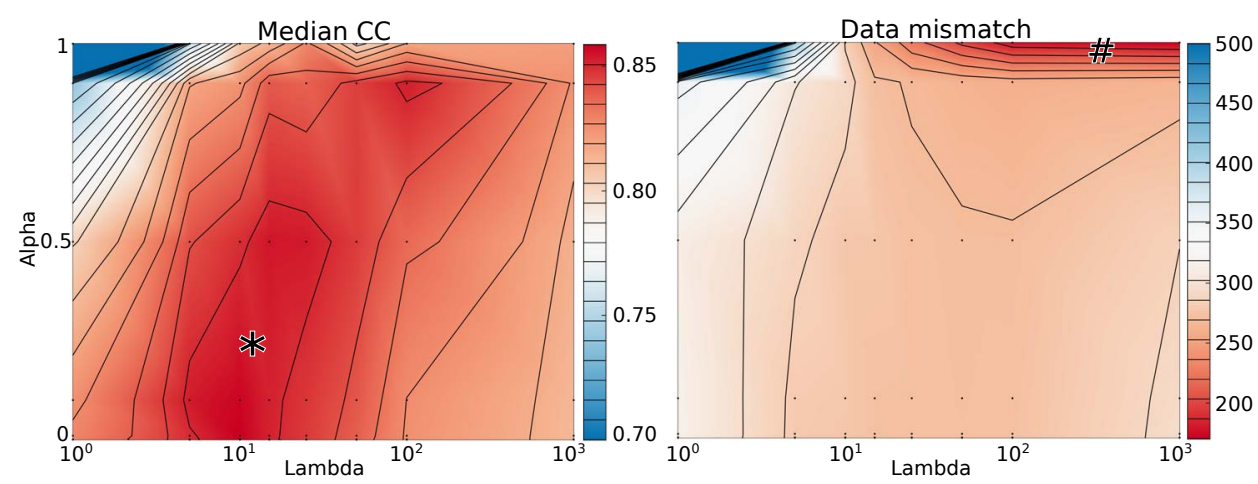

Figure 5.1: Dependency of in vivo results on elastic-net algorithm parameters, based on 8 recorded beats in a dog. For each combination of alpha and lambda, the median correlation coefficient (left) and data mismatch (right) is shown. Red color indicates optimal results. Grid search points are indicated by black dots.

\section{$5 \cdot 3$ Results}

Left panel of Figure 5.1 shows the results of the grid search for the optimal values of the parameters $\lambda$ and $\alpha$ of the multi-task elastic-net algorithm in equation (5.7). Highest accuracy (indicated by ${ }^{*}$ ) was obtained for $a=0.01$ and $\lambda=10$. These values were used for further analysis. Right panel of Figure 5.1 shows the results of the grid search based on data mismatch. The point of lowest mismatch (indicated by \#) was obtained for $\alpha=1$ and $\lambda=100$. Differences between the two optima were limited: $\mathrm{CC}=0.86$ for highest $\operatorname{accuracy}(*), \mathrm{CC}=0.82$ for lowest data mismatch $(\#)$.

Panel A of Figure 5.2 shows an example of reconstructed activation times on the ventricular epicardium for a sinus beat. For selected electrodes the corresponding electrograms are shown as recorded, Tikhonov-reconstructed and wavelet-domain elastic-net reconstructed. Panel B of Figure 5.2 shows similar results for a left-ventricular paced beat. In general, wavelet-domain elastic-net regularization was able to recover some details that were lost with Tikhonov regularization.

Figure 5.3 shows results for the full data set (5203 pairs of recorded and reconstructed electrograms). Columns show the results for the different reconstruction methods: Tikhonov regularization or wavelet-based regularization. Panel A shows accuracy of reconstructed epicardial potentials in terms of correlation coefficients between recorded and reconstructed electrograms. Wavelet-based regularization is able to recover more details in the electrograms, significantly improving reconstruction quality $(p<0.05)$. Panel B shows activation times and recovery times as reconstructed vs. recorded. Acti- 


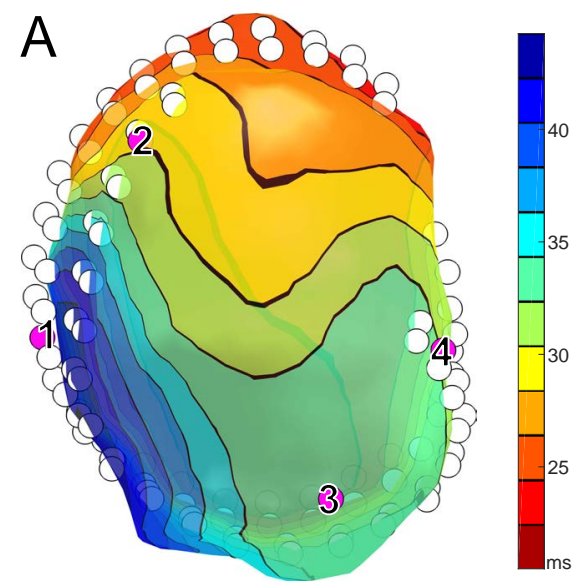

Recording Tikhonov Wavelet / Elastic net
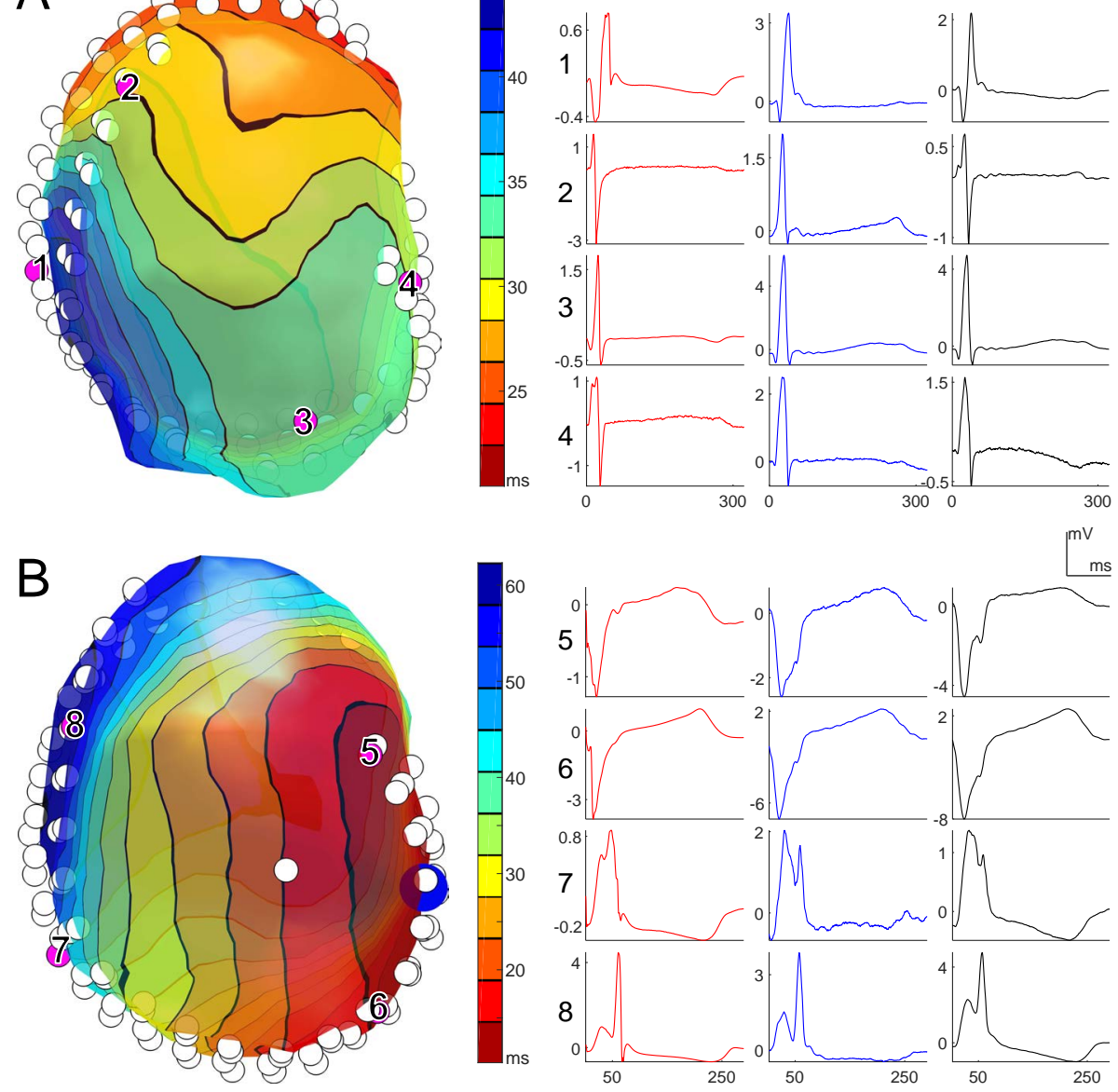

Figure 5.2: Ventricular epicardium (left, colored according to noninvasively reconstructed activation times) and recorded and reconstructed electrograms (right) during a sinus beat (panel A) and a left-ventricular paced beat (panel B, pacing location indicated by blue sphere). White circles represent the implanted epicardial electrodes. For selected electrodes (purple, numbered) the corresponding electrograms are shown: recorded (red), Tikhonovreconstructed (blue) and wavelet-domain elastic-net reconstructed (black). 


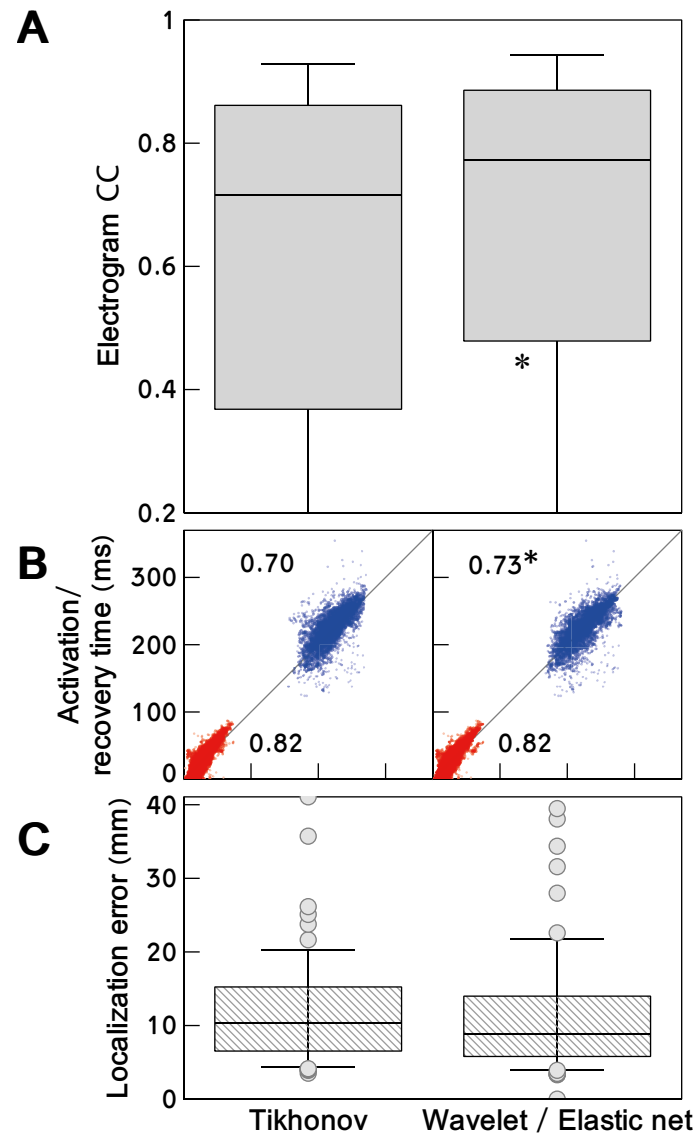

Figure 5.3: Results for the full data set. Columns show the result for the different reconstruction methods: traditional Tikhonov regularization, or wavelet-domain elastic-net regularization. Panel A: box plots of correlation coefficients between recorded and reconstructed electrograms. Box spans the interquartile range (IQR), i.e., the $25-75 \%$ range; median indicated by horizontal line; whiskers at 9-91\% range. Wavelet-based regularization improves reconstruction quality. Panel B: Activation times (red) and recovery times (blue) as reconstructed (horizontal axes) vs. recorded (vertical axes). Recovery times, especially, are improved by wavelet-based regularization. Panel C: Localization error between detected and known origins of pacing. An asterisk $\left({ }^{*}\right)$ indicates significant improvement compared to Tikhonov results. 
vation times (red) are not improved by wavelet-based regularization, but recovery times are $(p<0.05)$. Panel $\mathrm{C}$ shows localization error between detected and known origins of pacing. There is not significant difference between the two regularization methods.

\subsection{Discussion}

We have introduced a new method to regularize the inverse problem of electrocardiography in both time-frequency and space. This method pursues a sparse representation of the reconstructed epicardial potentials in the wavelet domain. By choosing a suitable wavelet basis, the main morphological characteristics of the expected epicardial electrograms can be expressed sparsely, i.e., in only a few non-zero wavelet coefficients. This likely reduces the influence of ill-posedness. Sparsity was promoted by a multi-task elastic-net approach. We evaluated this method on a series of uniquely-instrumented canine experiments, in which invasive epicardial recordings were obtained.

Epicardial potentials reconstructed with the wavelet-domain elastic-net approach attain a higher correlation coefficient compared to traditional zeroth-order Tikhonov regularization. More importantly, recovery times are reconstructed with higher accuracy. Abnormalities in recovery times can be an important substrate for cardiac arrhythmias and sudden cardiac death. Improved recovery time imaging might thus improve clinical care.

Activation time imaging is not improved and consequently localization of beat origin (which was based on the earliest activation time) is not improved either.

The multi-task elastic net returns the inverse solution which obtains a sparse solution over all wavelet coefficients simultaneously. This means that optimization is performed over both time-frequency (columns of $P_{H}$, wavelet coefficient index) and pseudo-space (rows of $P_{H}$, the epicardial nodes). The spatial relation between the epicardial nodes is implicitly included in this process, as this relation is captured by the transfer matrix $A$. However, this relationship could be exploited even more if one could define a wavelet transform over the irregularly curved heart surface.

To the best of our knowledge, spatiotemporal sparsity has not been pursued previously in a regularization method for ECGI. Greensite SVD applies spatiotemporal regularization (without pursuing sparsity) [95], but was outperformed by zeroth-order Tikhonov regularization in a previous study by our group [97].

Additionally, to the best of our knowledge, wavelets have not been applied in regularization of the inverse problem of electrocardiography. One advantage of wavelet-based regularization is the freedom in choosing the wavelet basis. Here, we choose to apply the Daubechies-2 wavelet transform, which was previously shown to be a good choice 
for sparse representation of heart-surface potentials amongst orthogonal wavelet transforms [126]. However, improved results could be expected for different choices for the wavelet basis. Designed wavelets could be used to tailor this method to specific situations. [127] For example, if one is interested in fractionated electrograms (which could occur after myocardial infarction), one could employ a more fractionated wavelet. Additionally, abnormally long or short activation or recovery durations might be captured with specifically designed wavelets. We were not able to test this in vivo, as the dogs from this experiment had healthy hearts.

\subsection{Conclusion}

We have introduced a novel method to regularize the inverse problem of electrocardiography. By simultaneously pursuing a sparse wavelet representation in time-frequency and exploiting correlations in space, epicardial potentials were noninvasively reconstructed with higher accuracy than with Tikhonov zeroth-order regularization. This indicates that sparse representations of the cardiac source can help to improve reconstruction accuracy in electrocardiographic imaging. Most notably, our approach led to improved imaging of recovery times. As abnormalities in recovery are an important substrate for cardiac arrhythmias and sudden cardiac death, these improvements could directly impact clinical interpretation.

\section{ACKNOWLedgment}

The authors would like to thank Dana Brooks, PhD, Jaume Coll-Font, MSc (Northeastern University, Boston, MA, USA), and Burak Erem, PhD (Harvard Medical School and Boston Children's Hospital, MA, USA), for kindly sharing their algorithm for spatiotemporal activation time approximation.

\section{CONFLICTS OF INTEREST}

Conflict of interest: none declared 


\section{AbStract}

Background: Exaggerated spatial and temporal dispersion of repolarization are well-known substrates for cardiac arrhythmias. However, the presence and extent of proarrhythmic repolarization heterogeneity are not easily detectable on the 12-lead electrocardiogram. Noninvasive imaging of electrical activation and recovery at the epicardium may provide mechanistic and diagnostic insights in such conditions. Here, we combine electrocardiographic imaging (ECGI) with computational modeling of human cardiac myocyte function to explain proarrhythmia in a female with sudden cardiac arrest due to primary ventricular fibrillation (VF).

Methods: The 47-year old female presented with polymorphic ventricular tachycardia (VT) and VF, without overt structural heart disease. Upon resuscitation, frequent short-coupled ventricular extrasystolic (VES) beats and normal QT intervals were noted during sinus rhythm. Based on 184 simultaneously recorded body-surface ECGs and a torso-heart geometry (digitized from a thoracic CT scan of the patient), local epicardial potentials, electrograms and isochrones were computed. Abnormal impulse formation was investigated by simulating human Purkinje, ventricular and atrial action potentials (APs) upon incorporating the effects of the TRPM4 genetic variant c.2531G>A, p.(Gly844Asp), identified in this patient.

Results: Epicardial isochrones showed normal ventricular activation during sinus rhythm, but abnormally short activation-recovery intervals $(180 \mathrm{~ms})$ at the right ventricular outflow tract (RVOT) and steep RV repolarization gradients. Short-coupled VES beats originated from the RVOT, and AP simulations suggested enhanced automaticity in Purkinje fibers, due to the $\mathrm{TRPM}_{4}$ mutation, as a contributor to their genesis.

Conclusion: Our translational approach, based on the combination of noninvasive ECGI at the organ level and AP simulations at the cellular level, helps to infer arrhythmia mechanisms and treatment options in cardiogenetic patients. In this case, short-coupled VES beats infringe on an apparently latent substrate of abnormally short and dispersed repolarization to potentially initiate VT and VF. Antiarrhythmic treatment with quinidine, a class-IA agent, appears very effective.

Adapted from:

1) MJM Cluitmans, J Heijman, RMA ter Bekke, RLM Peeters, RL Westra, HJGM Crijns, PGA Volders. Integration of noninvasive imaging of repolarization with cellular modeling in a clinical case. Unpublished.

2) MJM Cluitmans, PGA Volders. Noninvasive imaging of repolarization gives insight in arrhythmogenic substrate. Nederlandse Vereniging voor Cardiologie (NVVC) Voorjaarscongres, Noordwijk, the Netherlands, 9-10 April 2015. 


\subsection{INTRODUCTION}

Abnormalities in electrical recovery (i.e., repolarization) of the heart can form an important substrate for cardiac arrhythmias. On the 12-lead ECG these may appear as prolongation or shortening of the QT interval [131]; increased QT dispersion (i.e., difference between the minimum and maximum QT interval in each of the leads) [131]; beat-tobeat QT-interval variability [132], changes in T-wave morphology [131]; T-wave inversion [133]; T-wave alternans [131]; increased $\mathrm{T}_{\text {peak }}-\mathrm{T}_{\text {end }}$ or T-wave right slope [134]; early repolarization (i.e., elevation of the QRS-ST junction from baseline) [135], [136]; and short-coupled ventricular extrasystoles (VES) [137].

However, most of these parameters are poor predictors of arrhythmic outcome, as they lack significant sensitivity and specificity. Furthermore, the accuracy to detect repolarization abnormalities is limited by the spatial resolution of the 12-lead ECG. For example, increased QT dispersion is suggestive of steep spatial repolarization gradients in the heart. Neighboring areas with different repolarization durations can provide a substrate for reentrant tachycardia and reduce the threshold for ventricular fibrillation (VF). However, proarrhythmic repolarization dispersion may occur in a small myocardial region, which is not reflected by QT dispersion on the ECG. Conversely, distant repolarization differences in the heart may appear as QT dispersion, but will likely not create a proarrhythmic substrate. [131]

Noninvasive electrocardiographic imaging (ECGI) allows to reconstruct potentials, electrograms, and activation/recovery isochrones on the epicardium (Figure 1.12). [99] It can overcome the low spatial resolution of the 12-lead ECG by reconstructing electrograms directly at the epicardium from a much larger set of (up to 256) body-surface electrograms combined with computed tomography (CT)-derived information about the heart and body-surface geometries. Because of the increased spatial resolution and anatomical information provided by ECGI at the organ level, it may be better suited to detect regional repolarization abnormalities.

At the cellular level, the QT-interval is largely determined by the action potential duration (APD) of ventricular cardiomyocytes. Regional APD differences between the left ventricle ( $\mathrm{LV}$ ) and right ventricle (RV), between apex and base, and between the different layers of the ventricular wall (from endo to epicardium) largely determine the QT interval and T-wave morphology. These APD differences result from heterogeneous expression of multiple ion channels and can be exacerbated by genetic or acquired factors, thereby promoting repolarization dispersion. Computational modeling has proven useful to analyze the potential proarrhythmic effects of ion-channel dysfunction under a variety of conditions. For example, using a computational model of the canine ventricular 
cardiomyocyte, we have previously simulated cellular effects of beta-adrenergic receptor stimulation and demonstrated how loss-of-function mutations in the slow delayedrectifier $\mathrm{K}^{+}$-current $\left(\mathrm{I}_{\mathrm{Ks}}\right)$ selectively prolong $\mathrm{APD}$ during conditions of elevated betaadrenergic tone [138].

In this Chapter, we present a patient with sudden cardiac arrest due to primary VF, in whom local repolarization abnormalities were imaged with ECGI. In addition, we used computational modeling to investigate the potential proarrhythmic effects of a genetic variant in the transient-receptor-potential melastatin-related (TRPM) type-4 channel that was identified in this patient.

\subsection{PATiENT}

A 47-year old woman, without previous history of cardiovascular disease, presented with out-of-hospital syncopes and in-hospital cardiac arrest due to polymorphic ventricular tachycardia (VT) and VF, see Figure 6.1A. After defibrillation, she exhibited sinus rhythm with frequent, short-coupled VES beats (Figure 6.1B, QRS width $120 \mathrm{~ms}$ ) and short runs of non-sustained VT (NSVT, Figure 6.1 C). Her coronary angiogram showed no abnormalities and plasma electrolyte concentrations were normal at the time of presentation. No structural abnormalities were found with cardiac magnetic resonance imaging (MRI) and ultrasound. A 24-hours Holter recording showed 17,675 VES beats with short coupling interval and morphologies suggestive of origins in the RV wall. The patient used herbal tea and dietary supplements; discontinuation of these products did not reduce the number of short-coupled extra-systolic beats. Ajmaline provocation testing was negative for Brugada syndrome, but abolished VES beats completely. A subsequent electrophysiologic (EP) study confirmed the RV origins of VES beats, and showed normal endocardial RV voltage maps. Thus, conventional imaging did not provide evidence for the presence of a vulnerable substrate. In parallel, cardiogenetic screening was initiated to determine potential genetic factors involved in the arrhythmogenic phenotype.

\subsection{Noninvasive ECGI}

Noninvasive ECGI was performed in this patient (Figure 6.2). Epicardial isochrones showed normal ventricular activation during sinus rhythm (panel A1), with first epicardial breakthroughs (activation) at the RV outflow tract (RVOT) and RV apex. This activation pattern is consistent with normal patterns of activation as imaged previously 


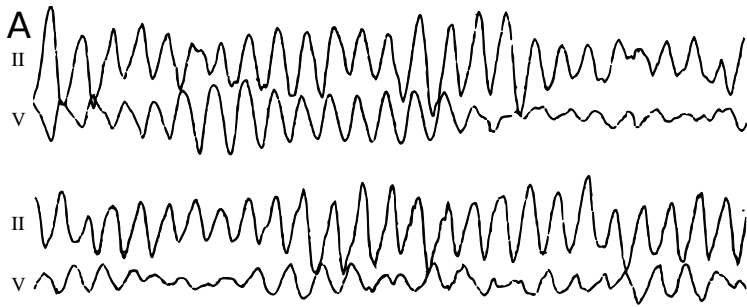

B

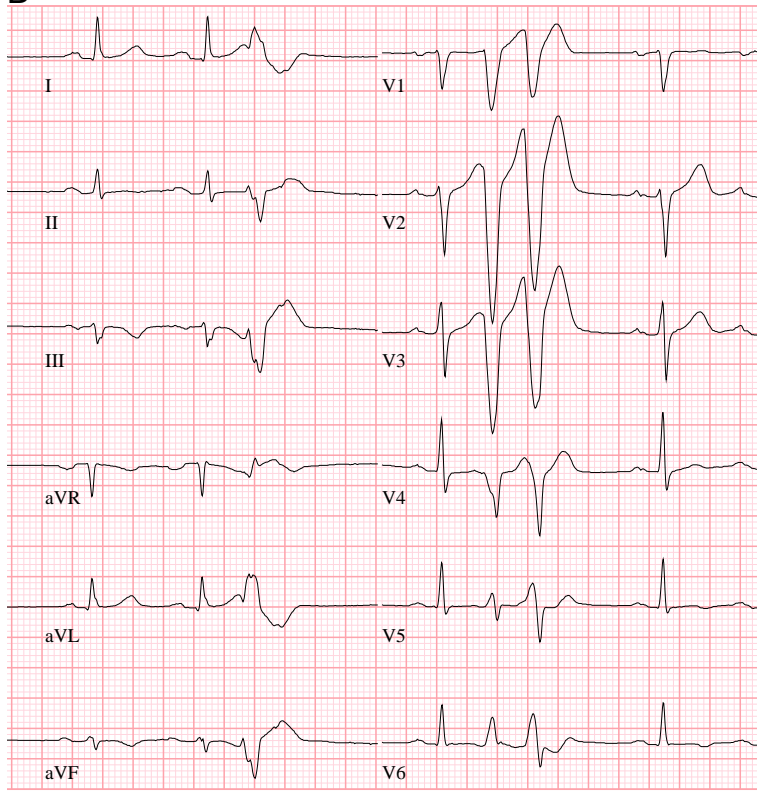

C
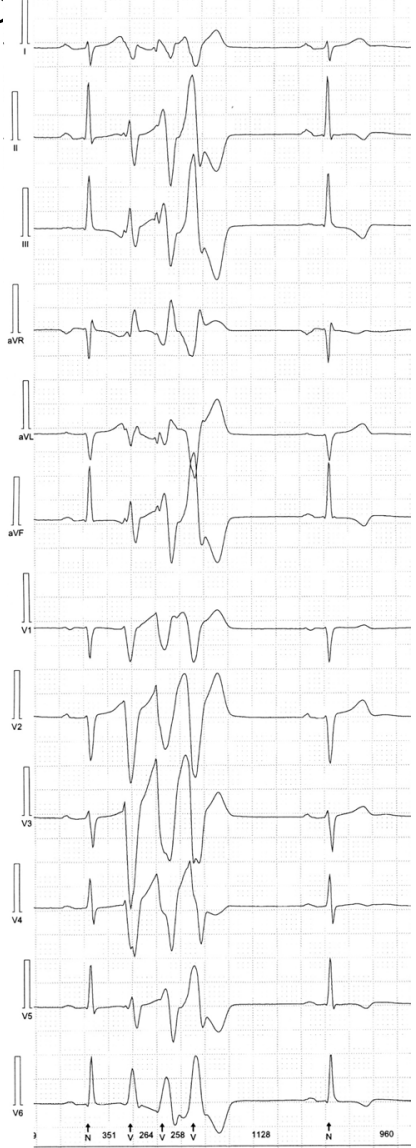

Figure 6.1: Polymorphic VT at presentation (A), short-coupled extrasystoles (B) and short runs of NSVT (C) in a 47-year old female patient with no previous cardiac history.

with ECGI in healthy human adults [42] and as shown by Durrer et al. in explanted human hearts [1]. Repolarization of this same sinus beat, on the other hand, was abnormal (panel A2). In particular, the RVOT region recovered abnormally early, being $\geq 180 \mathrm{~ms}$ from QRS onset. In normal conditions, epicardial recovery times are approximately $260-$ $360 \mathrm{~ms}$ in healthy subjects [42], compared to $180-340 \mathrm{~ms}$ in this patient. As a result, there was a steep repolarization gradient between the area of abnormally early recovery ( 180 $\mathrm{ms}$ at the RVOT) and normal recovery ( $300 \mathrm{~ms}$ at the inferior RV), which was $55 \mathrm{~ms} / \mathrm{cm}$, i.e., significantly larger than normal values of $2 \mathrm{~ms} / \mathrm{cm}$ reported in previous ECGI studies [73]. This gradient occurs over a distance of only 2 centimeters.

Figure 6.3 shows selected epicardial electrograms during sinus rhythm. There are no 


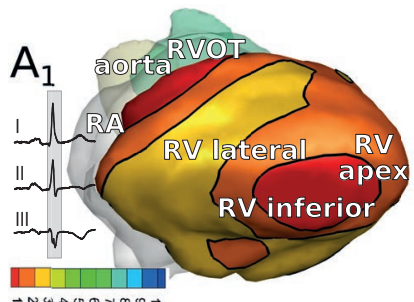

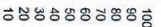

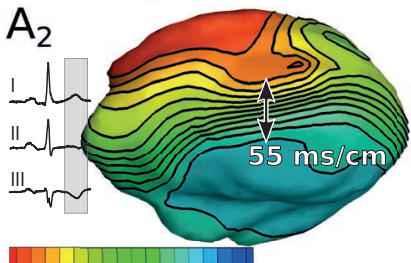

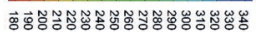

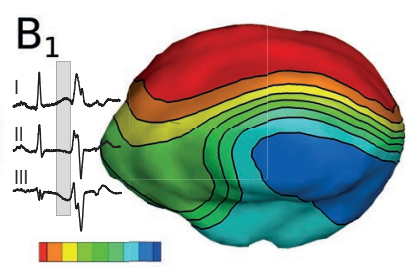

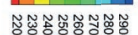

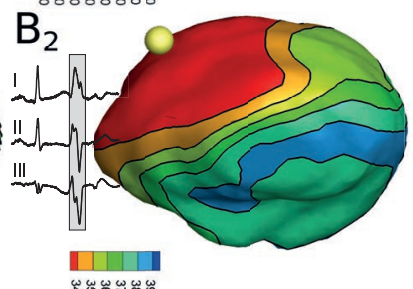

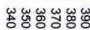

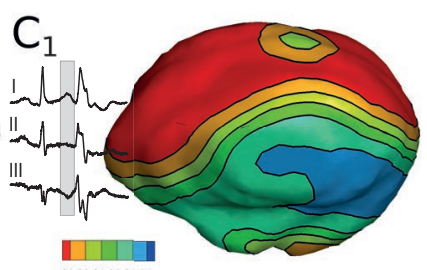

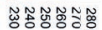

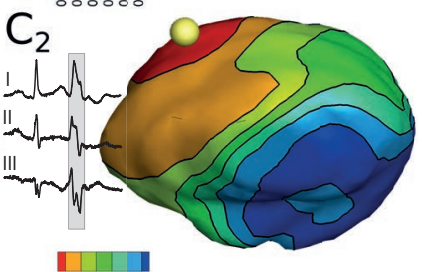

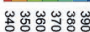

Figure 6.2: Noninvasively reconstructed epicardial isochrones for activation and recovery of sinus beats and ventricular extrasystolic beats. Epicardial isochrones show normal ventricular activation during sinus rhythm (panel A1), but abnormal recovery (panel A2) with steep repolarization gradients. VES activation (panels B2 and C2) follows the pattern of recovery (panels B1 and C1) of the preceding sinus beat. Yellow spheres in panels $\mathrm{C} 1$ and $\mathrm{C} 2$ indicate the origin of VES.

signs of abnormal conduction, i.e., no fractionation or ST elevation (the 'fractionation' in the lower electrodes is present throughout the full signal and is therefore likely to be noise, not fractionation due to conduction abnormalities).

The crowded isochrones are in a region where the local $T$ wave changes polarity. The epicardial $\mathrm{T}$ wave polarity depends on transmural and regional repolarization gradients and becomes (more) negative when regional dispersion increases [139], [140], such as in a region of steep repolarization gradient. In a previous ECGI study, crowded recovery isochrones were also observed in a region of $\mathrm{T}$ wave polarity change. [73]

VES beats in this patient were short-coupled to their preceding sinus beats, and usually they activated at the end of the preceding $T$ wave. ECGI shows that the VES beats originate from the RVOT region. The VES activation pattern (Figure 6.2, panels $\mathrm{B}_{2}, \mathrm{C}_{2}$ ) follows the pattern of recovery $\left(\mathrm{B}_{1}, \mathrm{C}_{1}\right)$ of the preceding sinus beat. Activation-recovery intervals (not shown) are similarly distributed as recovery times, indicating that repolarization gradients are induced by local changes in APD.

Although we were unable to record a VT-episode in this patient during the ECGI procedure, we hypothesize that the short-coupled VES beats infringe on a substrate of abnormally short (RVOT) and dispersed repolarization to potentially initiate VT and VF via functional reentry (Figure 6.4). Although ECGI provided insight in the electro- 


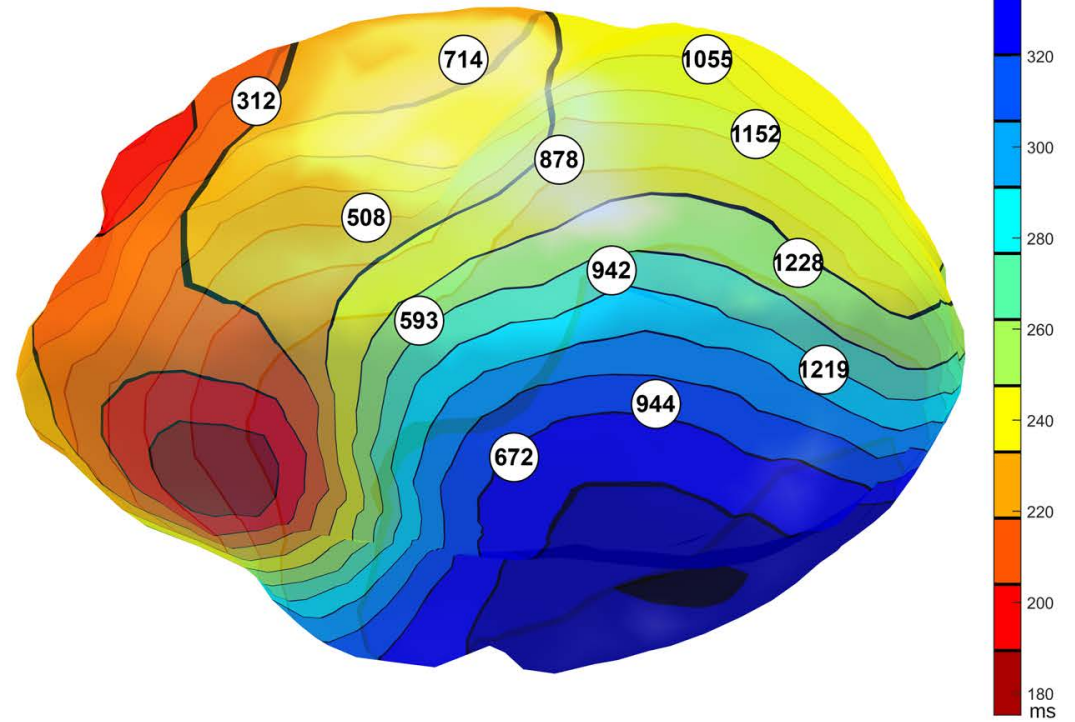

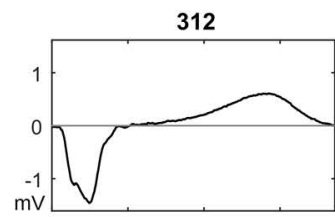

508

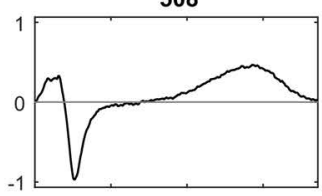

593

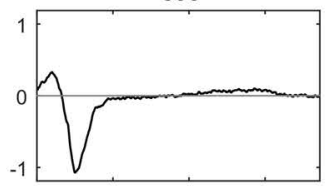

672

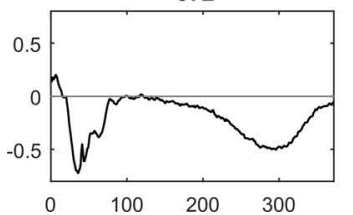

714

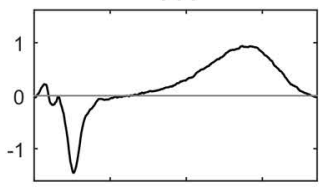

878

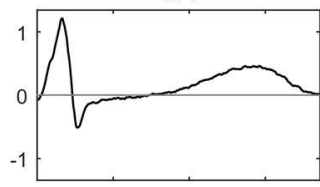

942
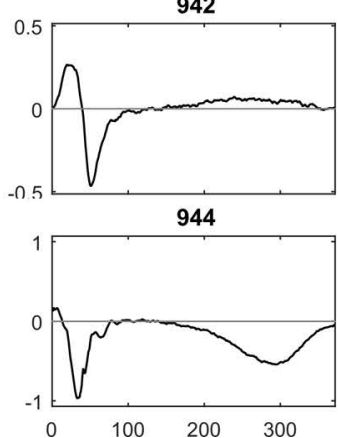

1055

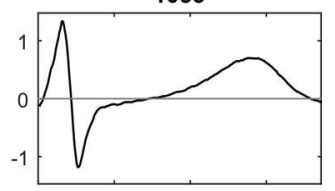

1152

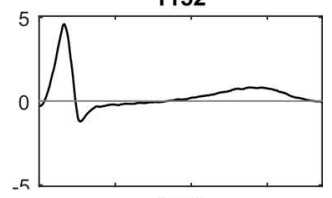

1228
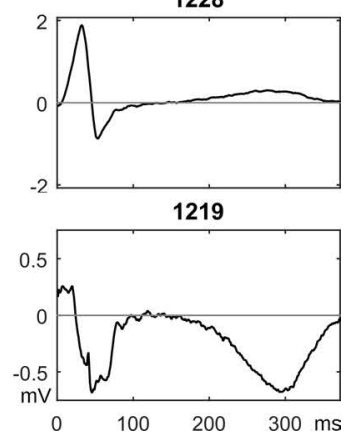

Figure 6.3: Epicardial isochrones of sinus recovery and selected epicardial electrograms (numbers reflect node id), as imaged with ECGI during a sinus beat. In the region of crowded recovery isochrones, there is a switch in local $\mathrm{T}$ wave polarity. 


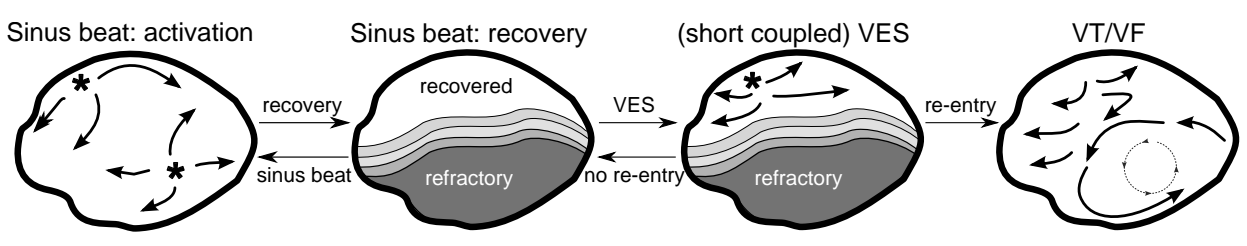

Figure 6.4: Hypothesis of VT initiation: short-coupled VES beat could initiate VT when late-recovering tissue is initially still refractory.

physiological substrate and the anatomical origin of the trigger, it does not yet provide an explanation for the genesis of the trigger.

\subsection{GENETIC ANALYSIS AND CELLULAR MODELING}

A broad range of genes involved in cardiac electrophysiology and arrhythmias was screened to identify potential molecular mechanisms involved in the electrophysiological substrate or the trigger in this patient. Two variants of unknown significance were identified.

The first variant, $c_{1} 188 \mathrm{C}>\mathrm{T}$ in the $\mathrm{CACNA} \mathrm{D}_{1} \mathrm{D}$ gene, predicted an alanine to valine substitution at position 63 (p.Ala63 Val) in the Cav1.3 protein that forms a lesser-known L-type $\mathrm{Ca}^{2+}$ channel $\alpha$-subunit. In the ventricles, the L-type $\mathrm{Ca}^{2+}$ channel is predominantly formed by a protein complex including the Cav1.2 subunit. The Cav1.3 subunit, on the other hand, is highly expressed in the brain, inner ear and in chromaffin cells involved in (nor)adrenaline secretion and regulation of the fight-or-flight response [141]. In the heart, Cav1.3 appears predominantly expressed in the sinoatrial and/or atrioventricular node, thus regulating pacemaker activity [142]. Furthermore, Cav1.3 appears to be moderately expressed in the human atria, but only very weakly in the human ventricle [142]. The alanine at position 63 in Cav1.3 is strongly conserved and the p.Ala63Val variant has not been described in the literature nor has it been found previously in the patient population tested at our institute. This variant was observed once in 121,366 alleles analyzed by the Exome Aggregation Consortium.

The second variant of unknown significance, TRPM $4.2531 \mathrm{G}>\mathrm{A}$, predicted a glycine to aspartic acid substitution at position 844 of the TRPM 4 channel. TRP channels represent a large family of ion channels, which are ubiquitously expressed and regulated by a wide range of signals (pain, temperature, taste, pressure). TRPM 4 is indeed widely expressed, including in the human heart, with the highest mRNA levels observed in the conduction system, followed by the septum and RV, but with much lower expression levels in the right atrium or LV [143], [144]. TRPM4 is voltage dependent and 

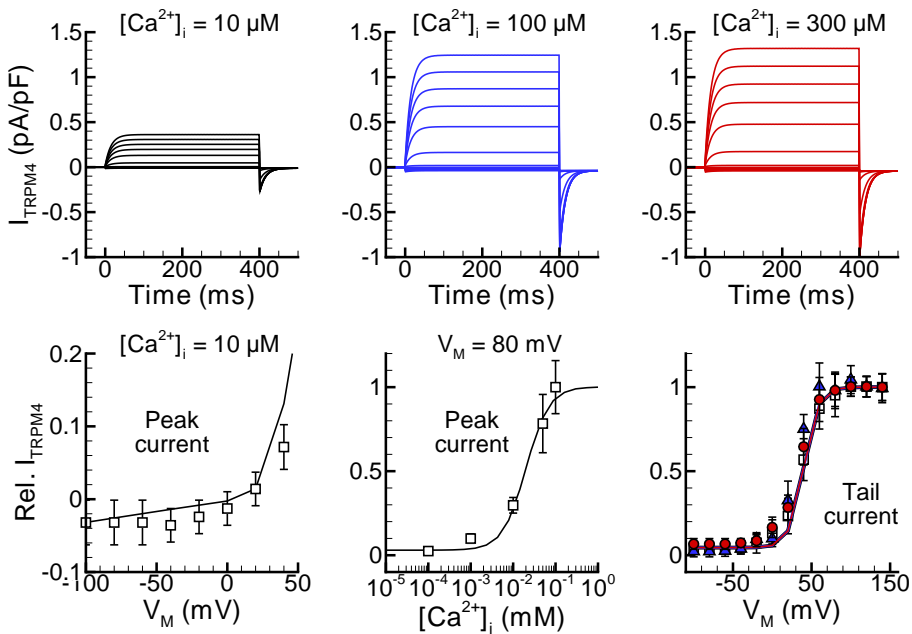

Figure 6.5: Validation of computational model of TRPM4 channel formulation. Top row shows simulated TRPM4 current during 400-ms depolarizing voltage-clamp pulses from - 80 to $+150 \mathrm{mV}$ at three different intracellular $\mathrm{Ca}^{2+}$ concentrations $(\mu \mathrm{mol} / \mathrm{L}$, black; $100 \mu \mathrm{mol} / \mathrm{L}$, blue; or $300 \mu \mathrm{mol} / \mathrm{L}$, red). Bottom row shows validation of simulated peak TRPM4 voltage dependence, $\mathrm{Ca}^{2+}$ dependence and tail current amplitude (lines) compared to experimental data (symbols, from [148], [149]).

regulated by intracellular $\mathrm{Ca}^{2+}[144]$. In its open state, the TRPM4 channel is similarly permeable to $\mathrm{Na}^{+}$and $\mathrm{K}^{+}$. The TRPM4 p.Gly844Asp variant is located in the second ABC-transport motif involved in ATP binding. It was observed 41 times in 19,480 alleles analyzed by the Exome Aggregation Consortium and has been associated in the literature with conduction block and Brugada syndrome [145]-[147]. The p.Gly844Asp variant results in increased current density due to increased channel expression at the plasma membrane.

Based on the known electrophysiological consequences of the TRPM4 p.Gly844Asp variant and the association between TRPM 4 and Brugada syndrome, an arrhythmogenic disorder previously associated with electrophysiological abnormalities in the RVOT, we decided to investigate the effect of increased TRPM 4 channel expression on cardiomyocyte electrophysiology in different regions of the heart using computational modeling. None of the published cardiomyocyte models include a formulation for TRPM 4 channels. We developed a Hodgkin-Huxley based formulation of TRPM4 encompassing a voltage-dependent gate and a $\mathrm{Ca}^{2+}$-dependent gate. Model parameters were fit to experimental data from the literature (Figure 6.5) and the channel was considered equally permeable to $\mathrm{Na}^{+}$and $\mathrm{K}^{+}$. The TRPM4 channel formulation was subsequently incorpo- 

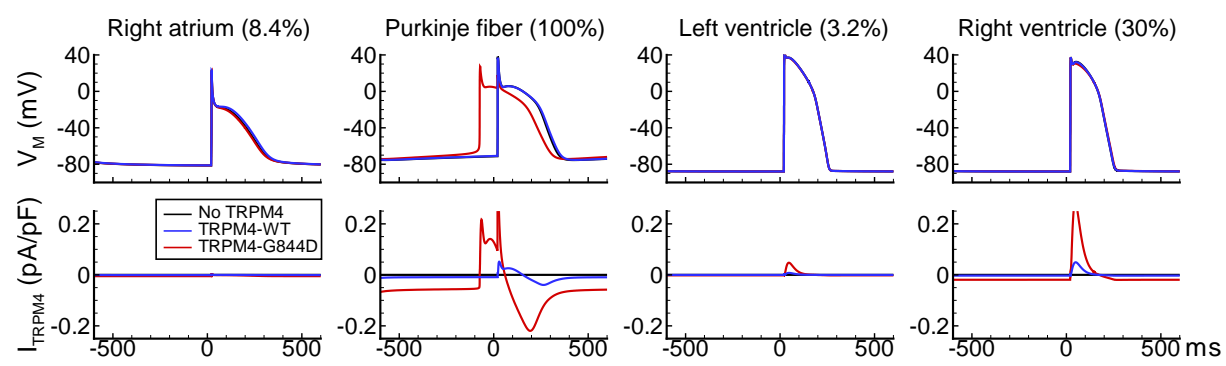

Figure 6.6: Simulated action potentials (APs, top panels) and TRPM4 current (bottom panels) in right atrial, Purkinje, left ventricular and right ventricular cardiomyocyte models in the absence of TRPM4 (black lines), using the wild-type TRPM4 formulation (TRPM4-WT, blue lines), or using the p.Gly844Asp formulation (TRPM4-G844D, red lines) with 6.3-fold increase in TRPM4 current. Numbers in top labels represent the relative TRPM4 expression in different parts of the heart (relative to the expression in the Purkinje fiber), based on [143].

rated into computational models of the human atrial cardiomyocyte [150], human Purkinje cell [151] and human ventricular cardiomyocyte [152], taking into account regional expression differences in TRPM4 [143]. The O'Hara et al. model of the human ventricular cardiomyocyte was originally developed to simulate LV cardiomyocytes. Given the RV origin of the ectopic activity and repolarization abnormalities in our patient, we developed an RV version of this model based on published LV-to-RV differences in ion channel expression [153]. Each model was simulated in the absence of TRPM4, with simulated wild-type $\mathrm{TRPM}_{4}$ current, or in the presence of the simulated p.Gly844Asp TRPM4 current during pacing at $1 \mathrm{~Hz}$ using the Myokit simulation package [115].

Both the wild-type and p.Gly844Asp TRPM 4 currents had very minor effects on simulated atrial and ventricular action potentials (Figure 6.6). By contrast, the large mutant $T R P M_{4}$ current (but not the wild-type version) significantly altered electrophysiological activity in the model of the human Purkinje cell. In particular, the inward current through $T R P M_{4}$ channels promoted diastolic depolarization and enhanced automaticity in this cell type.

\subsection{Discussion}

Hypotheses about the mechanisms of arrhythmia are often based on 1) abnormal repolarization, where spatial heterogeneity of APD can promte reentry; or 2) abnormal conduction, where tissue abnormalities (e.g., scar tissue) lead to slow and discontinuous AP propagation, which can also promote reentry. [154] In this case, ECGI showed signs of abnormal repolarization, in particular abnormal local early repolarization and steep repolarization gradients. Reconstructed epicardial electrograms did not show abnormal con- 
duction (e.g., fractionation, ST-segment elevation), nor did invasive endocardial voltage mapping. Therefore, abnormal repolarization, rather than abnormal conduction, likely creates the vulnerable substrate promoting VT in this patient.

A potential mechanism of VT initiation in this case is illustrated in Figure 6.4. The steep local repolarization gradient could function as line of conduction block for shortcoupled beats, facilitating functional reentry. Timing of the short-coupled beat is essential for reentry: very early automaticity will find no excitable tissue, whereas all tissue will be excited if automaticity comes too late. Only when timing is such that tissue is still partially refractory, functional reentry may occur. Ultimately, the patient was treated with quinidine, a class-IA antiarrhythmic agent, which successfully abolished VES beats (pre-quinidine: 17,675/24hrs, post-quinidine: 1/24hrs), and received an implantable cardioverter defibrillator (ICD). She was event-free at one-year follow-up.

Modeling of the TRPM4 mutation suggested that this mutation may promote increased automaticity in Purkinje myocytes. This could potentially explain spontaneous VES beats. However, in healthy hearts, automaticity from the conduction system yields narrow-complex beats. In this patient, the short-coupled VES beats showed a broad QRS morphology, indicative for an origin outside of the conduction system. Three hypotheses could explain this apparent discrepancy. First of all, it is known that in some cases there is rate-dependent partial blockade of the conduction system. Secondly, impulse formation from the Purkinje system might be so early that most myocardial tissue is still refractory, and the impulse can only exit at the region of early recovery. This would give rise to a broad-complex activation from that location. Thirdly, although single-cell AP simulations show that only Purkinje myocytes are likely to have increased automaticity, RV tissue also has a high presence of $\mathrm{TRPM}_{4}$, and regional differences or cell-to-cell coupling might give rise to RV automaticity. Currently, we have no data supporting any of these hypotheses. Future studies should investigate them further.

Treatment with quinidine abolished virtually all VES activity in this patient. In the AP simulations, abnormal TRPM4 automaticity was suppressed in the presence of simulated quinidine (simulated as a reduction in $\mathrm{I}_{\mathrm{Kr}}, \mathrm{I}_{\text {to }}, \mathrm{I}_{\mathrm{Na}}, \mathrm{I}_{\mathrm{Kp}}$ and $\mathrm{I}_{\text {sus }}$ based on published $\mathrm{IC}_{50}$ values $\left.[155]-[158]\right)$, Figure 6.7. Furthermore, quinidine prolonged the APD, which could have an effect on the area of early recovery.

This is not the first case in which ECGI was successfully used to image recovery abnormalities as a substrate for arrhythmia. In long QT syndrome [73] and Brugada syndrome [154], ECGI showed that patients had abnormally steep gradients of repolarization. Repolarization gradients were steeper in symptomatic patients. [73] More importantly, ECGI has been used previously to characterize early recovery. [72] In that case 


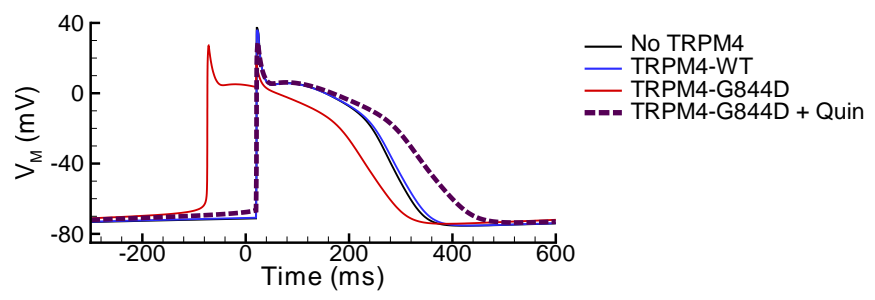

Figure 6.7: AP simulations in a Purkinje cell, showing that abnormal automaticity in the TRPM4 mutation is abolished in the presence of quinidine.

report, two patients with signs of early recovery on the 12-lead ECG were shown to have steep local repolarization gradients with local early repolarization. Also in these patients, VES beats originated in a region remote from the area with high repolarization dispersion. Our case study finds similar results, and adds a translational investigation with AP modeling of a mutation, providing a first hypothesis about the trigger and success of treatment with quinidine. Moreover, our case study shows that even when early recovery is not apparent on the 12-lead ECG, it can play a crucial role in arrhythmogenesis.

\subsubsection{Limitations}

Although ECGI provided a satisfactory characterization of the arrhythmia substrate, we could not provide an explanation for the presence of that substrate, and it remains unknown why the RVOT region recovered abnormally early. Unfortunately, we could not yet repeat ECGI after treatment with quinidine to investigate the effect on the repolarization gradient. Additionally, as no data was available on the other gene variant Cav1.3, we could not investigate its effect or any possible interactions with the TRPM4 variant. In general, this work provides several interesting hypotheses about arrhythmia mechanisms, but direct proof of these mechanisms could not yet be obtained.

\subsection{Conclusion}

This translational investigation, combining noninvasive electrical imaging at the organ level with AP simulations at the cell level, helped to examine the substrate and trigger of arrhythmogenesis in a patient with polymorphic VT and VF, suffering cardiac arrest. Noninvasive ECGI demonstrated repolarization abnormalities that were not apparent on the 12-lead ECG. AP simulations suggested enhanced automaticity due to the TRPM4 mutation, although it remains unknown whether this fully explains the broad-complex VES beats. This case shows that a translational approach may help to generate new hypotheses about arrhythmogenesis and treatment. 

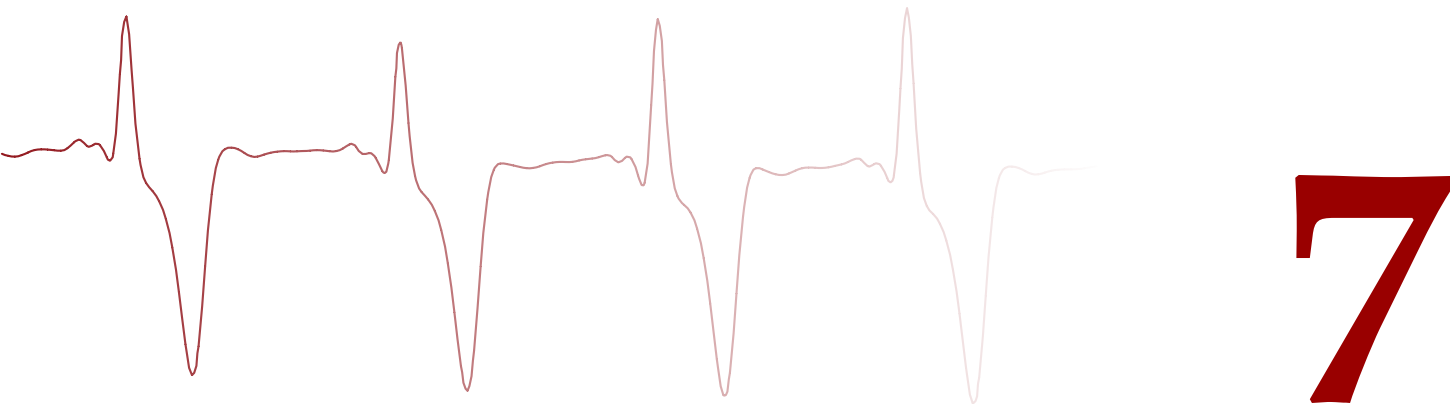

General discussion

\section{Contents}

Part I: Novel insights into ECGI . . . . . . . . . . . . 110

7.1 In vivo accuracy of ECGI $\ldots \ldots \ldots 110 \ldots \ldots$

7.2 The added value of spatiotemporal methods $\ldots \ldots \ldots 117$

7.3 The minimum number of body-surface electrodes $\ldots \ldots \ldots 121$

7.4 The added benefit of sparse data representation . . . . . . . . 124

7.5 Conclusions and future work $($ Part I $) \ldots \ldots \ldots \ldots$

Part II: Novel insights obtained with ECGI _ . . . . . . . . . . . 128

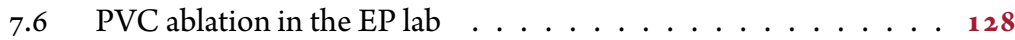

$7 \cdot 7$ Image integration $\ldots \ldots \ldots \ldots \ldots \ldots$

7.8 The role of recovery abnormalities in arrhythmia mechanisms . . 138

7.9 Conclusions and future work $($ Part II $) \ldots \ldots$ 


\section{Achieved in this thesis}

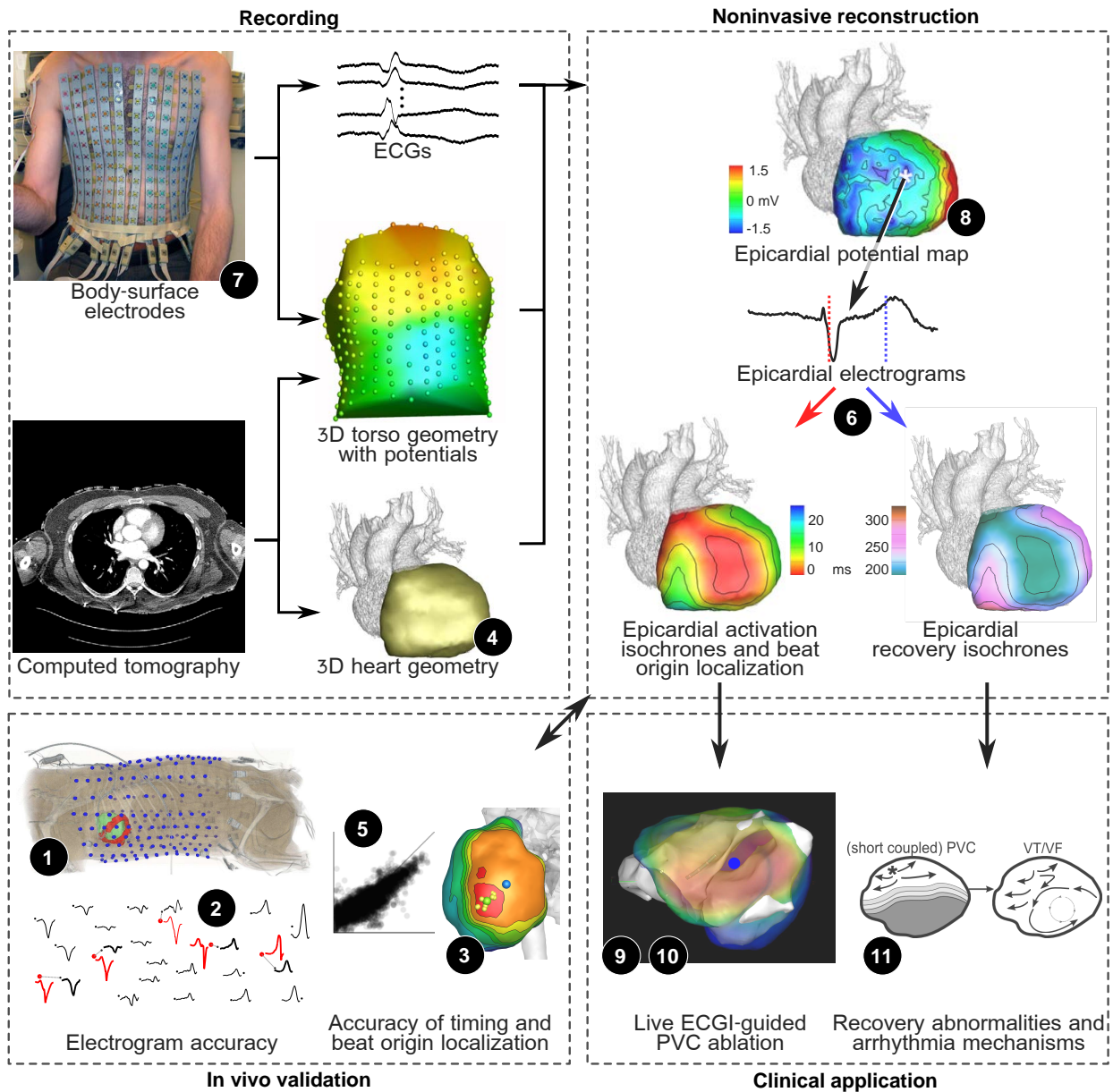


In this chapter, we put the results from this thesis in perspective. The discussion consists of two parts. The first part is dedicated to novel insights that were obtained about the technique and accuracy of ECGI. The second part of the discussion focuses on the scientific and clinical value of ECGI. Whereas ECGI as a method has the main stage in the first part, its applications are central in the second part. Both parts will not only give new insights based on data presented in the previous chapters, but also present new analyses and their implications.

The Figure on the previous page sketches the results obtained in this thesis, with the numbers reflecting the specific investigations that will be discussed in more detail:

1. The in vivo accuracy of noninvasively reconstructed electrograms on the epicardium (Section 7.1);

2. The influence of spatial mismatch on electrogram accuracy (Section 7.1.1);

3. The in vivo accuracy of beat-origin localization (Section 7.1.2);

4. The association between cardiac motion and reconstruction accuracy (Section 7.1.3);

5. The in vivo accuracy of activation/recovery-time estimation (Section 7.2);

6. The use of a spatiotemporal approach in activation/recovery-time estimation (Section 7.2);

7. The influence of the number of body-surface potentials on reconstruction accuracy (Section 7.3);

8. The improvements in reconstruction accuracy by using novel cardiac source models that aim for a sparse representation of potentials and electrograms (Section 7.4);

9. The localization of PVCs to guide ablation therapy (Section 7.6);

10. The integration of different imaging modalities to guide ablation therapy (Section 7.7);

11. The imaging of recovery abnormalities for arrhythmia mechanisms that are not apparent from the 12-lead ECG (Section 7.8).

Parts of this chapter have been published:

1) MJM Cluitmans, JMH Karel, P Bonizzi, MMJ de Jong, PGA Volders, RLM Peeters, RL Westra. In-vivo Evaluation of Reduced-Lead-Systems in Noninvasive Reconstruction and Localization of Cardiac Electrical Activity. Computing in Cardiology 2015, 42, 221-4.

2) MJM Cluitmans, J Coll-Font, B Erem, D Brooks, P Bonizzi, JMH Karel, PGA Volders, RLM Peeters, RL Westra. Spatiotemporal Activation Time Estimation Improves Noninvasive Localization of Cardiac Electrical Activity. Submitted. 


\section{Part I: Novel insights into ECGI}

This thesis has provided new insights into the accuracy of ECGI, the added value of spatiotemporal methods, and the role of cardiac motion. We will discuss these elements in the next sections. In addition, novel results based on new analyses will be presented, furthering spatiotemporal methods for activation/recovery-time estimation, and highlighting the influence of the number of body-surface electrodes on reconstruction accuracy. Finally, the use of sparse data representations as novel source models is discussed.

\subsection{IN VIVO ACCURACY OF ECGI}

In experiments with anesthetized dogs (Chapter 3 ), we obtained extensive and highquality recordings that were used to assess the accuracy of ECGI. These experiments allowed us to highlight the extent and role of spatial mismatch, investigate the accuracy and consistency of origin localization of paced beats, and quantify, for the first time, the accuracy of reconstructed activation and recovery times. Additionally, we showed that the accuracy of ECGI is negatively correlated with cardiac motion. We will elaborate on these elements in the following sections.

\subsubsection{THE ROLE OF SPATIAL MISMATCH}

One striking result is the role of spatial mismatch in electrogram variability. Direct comparison of recorded epicardial electrograms to the reconstructed electrogram at the closest virtual epicardial node showed an overall high accuracy (median correlation coefficient $[\mathrm{CC}]=0.71$ ) but with a considerable spread (interquartile range $=0.36-0.86$ ). We defined a 'good-enough' correlation as $\mathrm{CC}>0.70$, and at direct comparison $49 \%$ of the correlation coefficients was below this threshold. However, a good-enough $\mathrm{CC}>0.70$ with a reconstructed electrogram was found at some location on the heart surface for $84 \%$ of all recorded electrograms. For $77 \%$ of these, the virtual node with good-enough correlation was within $1 \mathrm{~cm}$ of the electrode, and for $90 \%$ within $2 \mathrm{~cm}$. This strongly indicates that a spatial mismatch, up to $2 \mathrm{~cm}$, explains a large part of the variability of the electrograms. The variability was most pronounced in areas where the electrograms change in polarity. This can be explained by the fact that a mismatch in polarity between the reconstructed and recorded electrogram has a strong negative effect on the corresponding correlation coefficient.

The spatial mismatch is visualized for three sinus beats in Figure 7.1. For each electrode with a spatial mismatch (i.e., direct comparison to the closest node yielded a CC $<0.70$ ), an arrow is drawn from the electrode to the closest node with a good-enough 


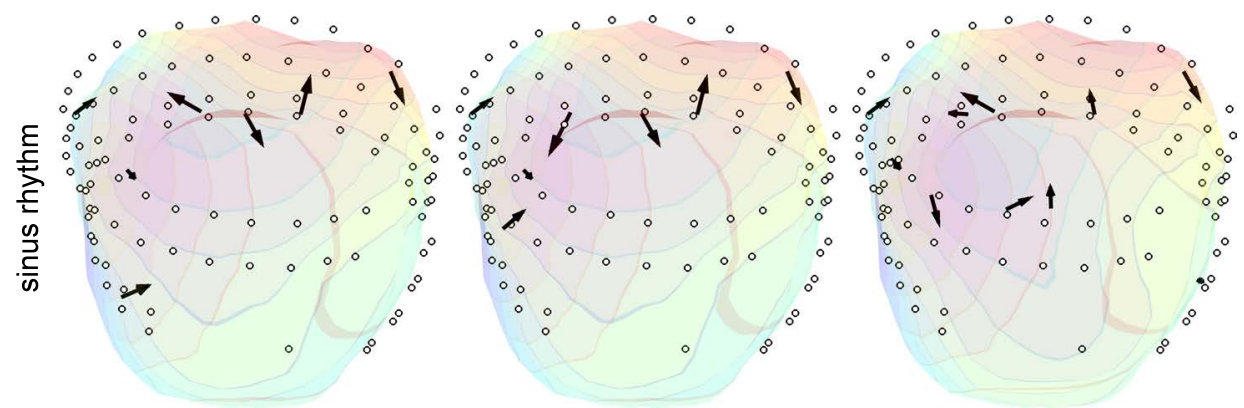

Figure 7.1: The direction of spatial mismatch between recorded and reconstructed electrograms during three sinus beats in a dog. Epicardial surface is colored according to noninvasively reconstructed activation time (red: early, blue: late) and is made semi-transparent. The circles indicate the implanted electrodes. For each electrode with a spatial mismatch, an arrow to the closest node with good-enough correlation (defined as $>0.70$ ) is shown.

correlation. The location and direction of the spatial mismatch are very similar for all three sinus beats. Results for paced beats are shown in Figure 7.2. Each row shows the spatial mismatch for beats paced at different, but very closely located electrodes. The location and direction of spatial mismatch are very similar between those beats (i.e., there is close resemblance between columns for a given row). However, the characteristics of spatial mismatch are very different between pacing regions that are far apart (i.e., little resemblance between different rows). Thus, spatial mismatch seems to be consistent with activation patterns. These results suggest that the differences in electrical (and possibly mechanical) activation play a role in the location and direction of spatial mismatch.

\section{CaUses of SPATIAL Mismatch}

The exact origins of spatial mismatch are unknown. In part, it will be influenced by the quality of the digitized torso-heart geometry. This geometry is segmented manually from a CT scan. Although the CT scans can be of high resolution (typically $0.75 \times 0.75 \mathrm{~mm}$, with variable slice thickness), the manual segmentation will be of lower quality. The effect of segmentation resolution has not been investigated by us or in literature.

Another factor is the accuracy of the forward model. The forward model, describing the propagation of the electromagnetic field from the heart to the body surface, is fully defined by the geometry and conductivity of the thoracic tissue. In our implementation, the torso is homogeneous (i.e., has equal conductivity at all locations), and the only volume of distinct conductivity is the heart. Other studies have investigated the effect of more realistic, complex torso inhomogeneities, e.g., including bone, fat, vessels and mus- 

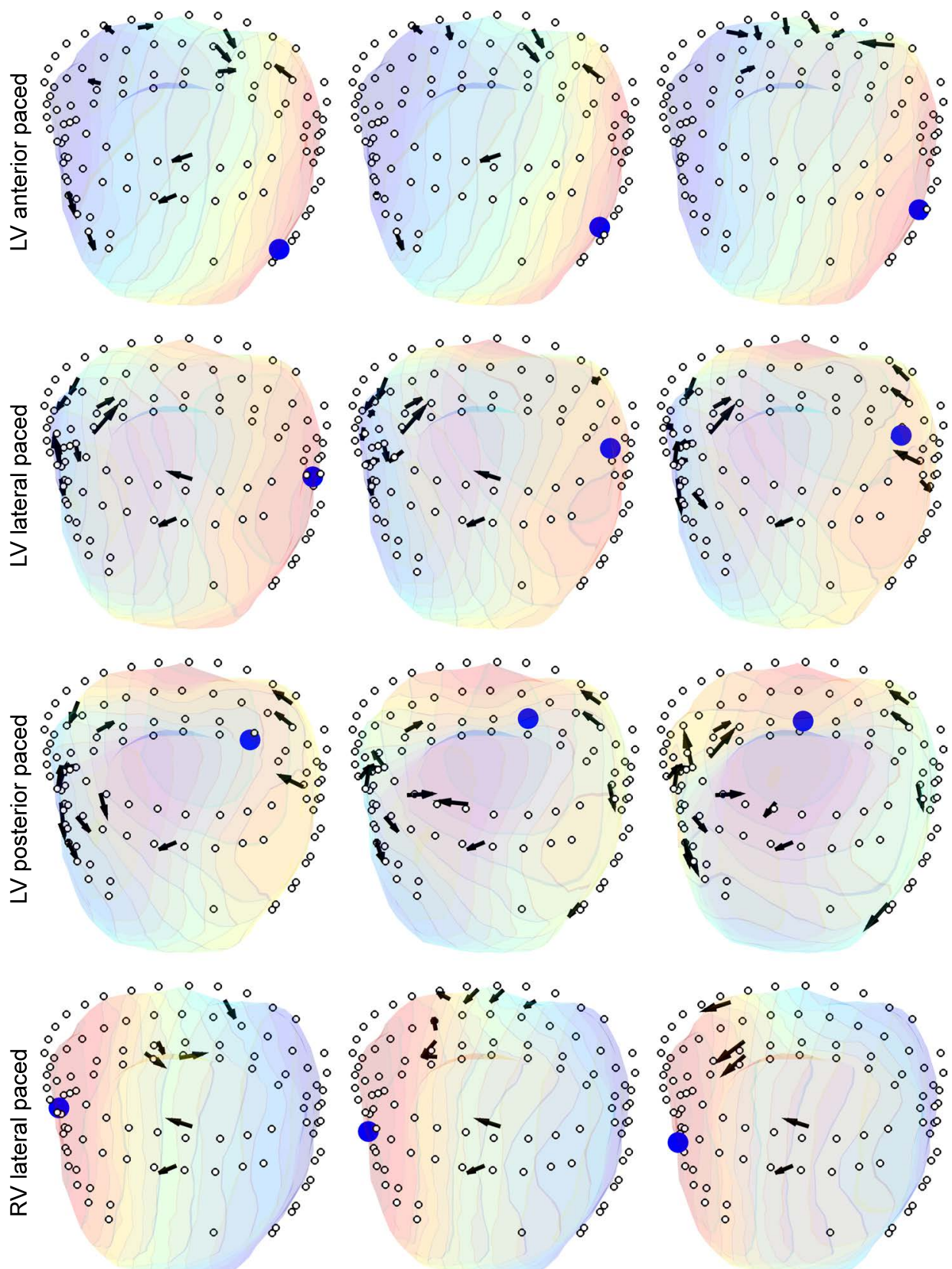

Figure 7.2: The direction of spatial mismatch for paced beats in a dog. Blue sphere indicates the pacing location. Each row shows beats paced from different but adjacent electrodes. Pacing locations are at the LV anterior (first row), LV lateral (second row), LV posterior (third row) and RV lateral wall (fourth row). 
cles. One would expect that a more realistic torso model results in more accurate reconstructions. However, with increasing complexity of the torso model, the ill-posedness increases as well (reflected by an increase in condition number of the transfer matrix [90]), and the reconstructions are more sensitive to the influence of noise. Whereas in silico studies often indicate that including the torso inhomogeinities increases accuracy [40], [88], [89], in vivo results suggest no benefit or even reduced accuracy [90], [159]. The difference between in silico and in vivo results could be explained by the larger amount of experimental control in the former, and the more realistic noise levels (on potential recording, geometry precision, and conductivity accuracy) in the latter. Recently, a highquality animal study similar to our study also investigated this aspect. Bear et al showed that the forward model with a more realistic, complex torso geometry did not improve in vivo forward solutions significantly with respect to body-surface potential distributions. [104] Their in vivo investigation of the inverse solution showed no benefit for including torso inhomogeneities either. [160]

Another source for spatial mismatch could be the assumption that the torso model is static, which ignores movement of the heart and torso. Usually, the heart is captured in a diastolic (relaxed) state by ECG gating during the CT scan. Additionally, the scans are obtained during breath-hold. Thus, both the effect of breathing (which changes the torso geometry, and the position of the heart within the torso), and the effect of cardiac contraction are ignored. These effects should be investigated further in future research. In Chapter 3 , we hypothesized that ignoring cardiac motion can result in a spatial shift of potential patterns, which could contribute to the observed spatial mismatch. However, cardiac motion is unlikely to be the only cause of this mismatch. If the spatial mismatch was only due to the assumption of a static cardiac geometry, one might expect a relation between the direction of spatial mismatch and the direction of electrical activation and mechanical contraction. However, such a relation is absent, as Figures 7.1 and 7.2 show that the directions of spatial mismatch are not uniformly aligned in the same direction. We will discuss the role of cardiac contraction in more detail in Section 7.1.3.

Additionally, the quality of the 'golden standard' epicardial recordings is dependent on many experimental factors, and might not always be that 'golden'. From Figures 7.1 and 7.2, it is clear that the spatial mismatch is not due to bad electrodes: a broken electrode would result in a consistently low-quality recording, and its quality would not change between pacing locations. Thus, the spatial mismatch would always occur on the same location, which is not the case. However, it is possible that some electrodes lose contact with the underlying tissue due to mechanical deformation to which the somewhat stiff electrode bands cannot adapt optimally. This would create loss of contact during the same type of mechanical activation (i.e., beats paced from closely related loca- 
tions), as is seen here. Although we carefully selected electrograms to be of sufficient quality (i.e., having good contact with the tissue), there is a gray zone between acceptable and unacceptable electrograms. However, it is unlikely that a bad recording, either due to a broken electrode or due to loss of connection during mechanical deformation, has a better correlation coefficient with another nearby node; it is more likely that no good correlation would be found at all.

We conclude that no definite single cause for spatial mismatch can be pinpointed, and the spatial mismatch is likely the combined result of many (or all) of the discussed factors: geometrical accuracy of the torso-heart model, accuracy of the forward/inverse model, including or ignoring torso inhomogeneities, the assumption of a static torso (ignoring breathing effects), the assumption of a static heart (ignoring cardiac deformation), and experimental influences (broken or disconnecting electrodes).

\section{IMPLICATIONS OF SPATIAL MISMATCH}

For clinical applications, it is important to know that spatial mismatch can be present, and electrogram abnormalities should be interpreted with this in mind. For example, local fractionation of electrograms, which can be a sign of myocardial scar tissue, can only be localized with confidence with $2 \mathrm{~cm}$ accuracy. This could be important when assessing the electrical consequences (imaged with ECGI) of scar tissue (e.g., imaged with MRI). Similarly, this spatial mismatch could explain the accuracy of origin-localization of paced beats, which in our study had a median error of $1 \mathrm{~cm}$, and $84 \%$ below $2 \mathrm{~cm}$.

During clinical applications, the spatial mismatch may be larger. In the animal experiments, the position of the animal was exactly identical during the CT scan and the body-surface potential (BSP) recordings. However, in patients the CT scan is obtained with the patient in a fully horizontal position, whereas the BSP recording is usually obtained in a semi-supine position. The BSPs are recorded in this position because it is more comfortable for the patient during the relatively long BSP recordings, and comfort is important to reduce movement artifacts. However, it is known that the position of the heart within the torso is different between a supine and semi-supine position. Thus, in clinical applications, it is likely that the position of the heart is (slightly) different during the recording of the electrical signals compared to the moment of geometrical acquisition. This effect should be investigated to determine its relevance for clinical applications.

\subsubsection{ACCURACY AND CONSISTENCY OF PVC LOCALIZATION}

As discussed in Chapters 2 and 3 , localization of paced beats is used as a surrogate for the localization of spontaneous ventricular extrasystolic (VES) beats, also known as prema- 
ture ventricular contractions (PVCs). The accuracy of (the potential-based implementations of) ECGI in localizing paced beats was investigated previously, both in torso-tank experiments [33], [101] and human experiments [35], [44], [57], [78], [101], see Table 3.1 (Chapter 3 , page 47). Our results are in agreement with these studies.

In addition, we have shown there that pacing localization is consistent for beats paced consecutively at the same location. The only difference in identical, consecutively recorded beats is noise and breathing artifacts. However, due to the ill-posed nature of the inverse problem, the inverse reconstruction process is very sensitive to noise, which could result in different reconstructions for these beats. In our results, the noninvasively reconstructed origins of these beats were within confined regions, indicating that the influences of noise and breathing artifacts were well addressed by regularization and did not have a large influence on localization consistency. Currently, international collaborations are ongoing to investigate the PVC-localization accuracy of different ECGI implementations. [161]

The accuracy of localization, and the consistency of accuracy, is important for clinical management. High incidence of PVCs can (further) deteriorate pump function during heart failure, cause palpitations, or increase risk of arrhythmias in the presence of substrate. For these reasons, PVCs can be the target of ablation therapy. Accurate and consistent localization of PVCs origins can thus play an important role in clinical therapy, as we will show for several cases in Part II of this Chapter.

\subsubsection{INFLUENCE OF CARDIAC MOTION}

Currently, cardiac motion is ignored in ECGI. The static torso-heart geometry is usually based on a CT scan taken during cardiac diastole, to capture the heart in a quiescent state. However, during the recording of body-surface potentials that are used for inverse reconstruction, the heart's geometry is changing, both in position within the torso, and in shape. This could partially, but not fully, explain the spatial mismatch, as discussed in Section 7.1.1 (page 111). In Chapter 3, we have shown that there is a negative correlation between the degree of movement of an implanted epicardial electrode and the accuracy of local reconstructed electrograms. However, we have only shown this for sinus beats. The mechanical contraction of a sinus beat is instantaneous and uniform, resulting in concentric movement of the heart. Beats that activate abnormally can result in non-uniform activation and non-concentric contraction, i.e., dyssynchrony, for example in left or right bundle branch block or PVCs. The simplification of a static heart may have the largest influence on the reconstruction of beats that result in non-concentric contraction. 


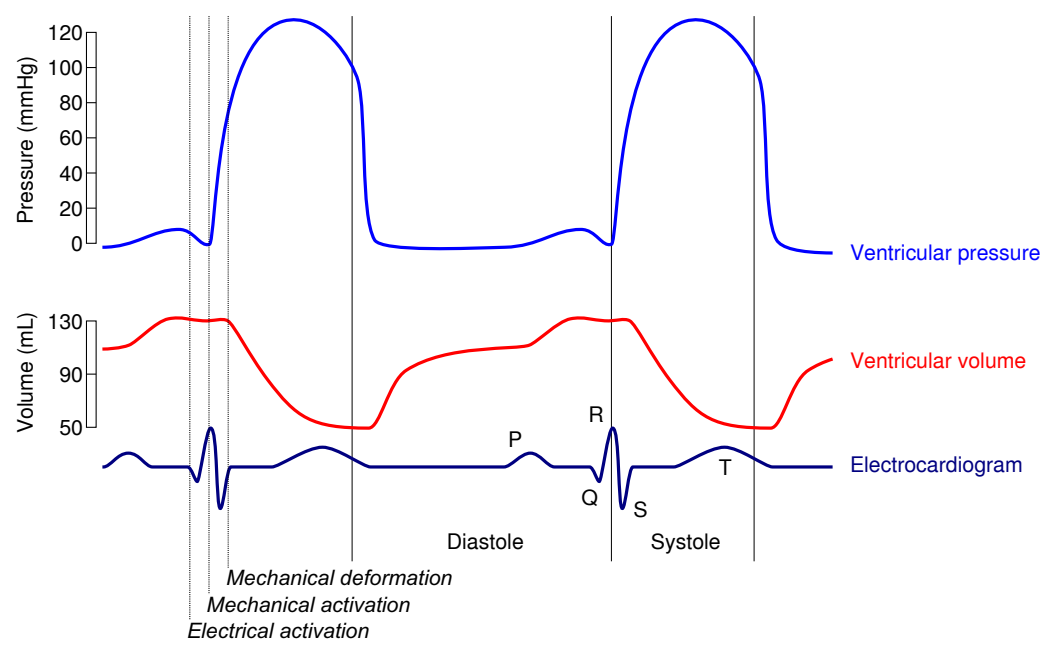

Figure 7.3: Wiggers diagram, showing the relation between electrical activation (visible on ECG) and mechanical activation (resulting in increase in ventricular pressure) and deformation (resulting in change in ventricular volume).

\section{CLINICAL IMPLicATIONS}

As there is some delay between electrical activation and mechanical contraction of cardiac tissue, the assumption of a static, diastolic geometry is likely of little influence on the reconstruction accuracy of the first part of a beat. Especially for normal sinus beats with quick electrical conduction, the QRS complex has already progressed halfway before mechanical contraction starts, and is almost finished when mechanical deformation occurs, see Figure 7.3. However, abnormal activation (e.g., due to bundle branch block or PVCs) can be slower, resulting in mechanical contraction and deformation during large parts of the QRS complex. Nevertheless, detection of beat origins (both normal and abnormal) is likely not influenced by the assumption of a static diastolic geometry, as it is only based on the initial activation of a beat during which there is no contraction yet.

On the other hand, the reconstruction of repolarization would likely suffer much more from the assumption of a static (diastolic) geometry, as we demonstrated for the patient in Figure 3 of Chapter 2, where we used both a diastolic and a systolic geometry for reconstructing the same body-surface potentials on the epicardium.

\section{FUTURE PERSPECTIVE}

Future studies, designed to detect the influence of cardiac motion, could provide convincing arguments that ECGI should incorporate dynamic changes of cardiac geometry. 
Cardiac mechanics could then be included by 'functional scanning' techniques. For example, CT and MRI are capable of capturing movement of the heart at different phases of the cardiac cycle, provided that the heart rhythm is regular. This allows to capture the change in cardiac geometry for sinus beats. For example, the CT protocol in MUMC+ allows for CT reconstructions at every $10 \%$ of the RR interval, effectively providing the cardiac geometry each $1 / 1$ oth of the cardiac cycle. However, manual segmentation of the epicardial geometry is currently very time consuming, and repeating this process ten times to capture the full movement of the heart is not practical. Therefore, techniques should be developed that automate parts of this process. One approach could be to manually segment a diastolic (fully relaxed) and systolic (fully contracted) epicardial surface, and interpolate the movement of the heart in-between these phases. However, this approach would not be able to capture asymmetric contraction (e.g., during bundle branch block or paced rhythm). A fully automated segmentation would provide a single geometry in which the position of the epicardial nodes is described as a continuous function of time, i.e., the geometry is morphed to capture the movement of the heart during each time instant.

To capture the movement of (usually sporadic) PVCs, MRI and CT are less convenient. Cardiac ultrasound may provide an alternative and can be used in combination with mechanical modeling to create a heart geometry with abnormal contraction.

\subsection{THE ADDED VALUE OF SPATIOTEMPORAL METHODS}

In the previous sections, the accuracy of ECGI was discussed, and causes for variability in electrogram accuracy were highlighted. In this section, the spatiotemporal technique to deal with electrogram variability is discussed, which allows more accurate reconstructions of activation times, recovery times, and beat origins. This technique was applied in Chapters 3-6 and this will be more closely examined in this section.

The accuracy of reconstructed electrograms is influenced by noise, spatial mismatch and other artifacts induced by ill-posedness. This affects the accuracy of estimation of activation and recovery times from reconstructed electrograms. Usually, activation and recovery are determined only based on temporal criteria, namely as the moment in a local electrogram with the steepest downslope (during the QRS complex) and the steepest upslope (during the $\mathrm{T}$ wave), respectively. However, fractionation can be present in the electrogram, due to the activation pattern, noise, or the influence of ill-posedness. It that case, it can be difficult to determine which deflection represents local activation. By incorporating information from neighboring electrograms, the most likely deflection can be chosen, as illustrated in Figure 7.4. This is exploited by a method proposed previously 


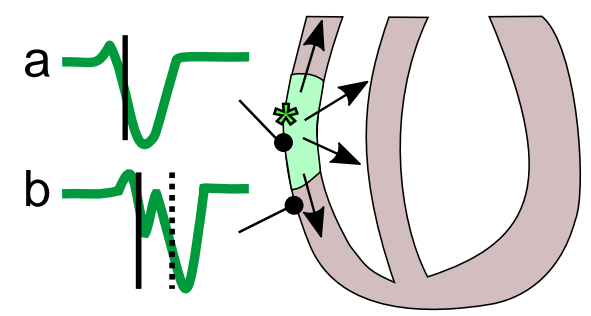

Figure 7.4: Illustration of the added value of spatial information in determining the moment of local activation. Electrogram $b$ is fractionated, with two deflections that could be selected by a temporal-only activation-time estimation method. Comparing this electrogram to neighboring electrogram $a$ makes the first deflection most likely the true activation time.

[100], which detects the simultaneous presence of the spatial and temporal features associated with a passing wavefront.

In the previous Chapters, this spatiotemporal approach was combined with spatial smoothing to remove outliers. This allowed us, for the first time, to assess the accuracy of activation and recovery timing quantitatively. Qualitatively, activation maps have been compared visually in previous studies, see Table 3.1 (Chapter 3, page 47). To the best of our knowledge, such comparisons do not exist for recovery maps. Moreover, quantitative comparison is important to obtain a more thorough understanding of the electrical substrate of arrhythmias. We showed that activation times, and to a lesser extent recovery times, could be determined with confidence when using a spatiotemporal approach.

In this section, we expand the results and analyses of previous chapters by presenting new investigations on the effect of the temporal-only criteria, the spatiotemporal approach and spatial smoothing.

\subsubsection{ESTIMATION OF ACTIVATION AND RECOVERY TIMES}

The temporal-only approach defined the moment of activation as the moment of steepest voltage downslope $(\max -\partial \varphi(t) / \partial t)$ during the QRS complex, where $\varphi(t)$ is the potential at the epicardial node under consideration at time $t$. Recovery times were defined as the moment of maximum $\partial \varphi(t) / \partial t$ during the $\mathrm{T}$ wave. These approaches were applied after smoothing the electrograms to reduce the influence of noise.

The spatiotemporal approach, proposed by Erem et al. [100], takes advantage of the spatial relationship between neighboring nodes and their potentials. They noted that not only the temporal signal (i.e., the local potential at a single node) changes quickly when an activation wavefront passes, but also the spatial gradient of potentials between neighboring nodes. More formally, for each epicardial node, they defined the activation 
time $\tau$ as:

$$
\tau=\min _{t}\left\{\|D \varphi(t)\|_{2} \cdot \frac{\partial \varphi(t)}{\partial t}\right\}
$$

where $\varphi(t)$ is the potential at the epicardial node under consideration at time $t, D \varphi(t)$ is the approximated spatial gradient, and $\partial \varphi(t) / \partial t$ the approximated temporal derivative. Although designed for activation-time estimation, we also applied this method to estimate recovery times; the minimization problem (equation 7.1 ) in this case becomes a maximization problem.

Golden standard activation and recovery times to compare with, were determined from recorded electrograms with the temporal-only method.

\subsubsection{ADDITIONAL SPATIOTEMPORAL SMOOTHING}

In addition, we explored the effects of the smoothing technique applied in reference [162]. This method smoothes estimates of activation or recovery times over the heart surface. The final times $\tau_{D}$ are a balance between the current noisy estimations $\tau$ and a minimization of the Laplacian function on the surface. This trade-off is obtained through the following minimization:

$$
\tau_{D}=\min _{\tau_{D}}\left\{\left\|\tau-\tau_{D}\right\|_{2}^{2}+\gamma\left\|L \tau_{D}\right\|_{2}^{2}\right\}
$$

The first term, $\left\|\tau-\tau_{D}\right\|_{2}^{2}$, corresponds to a least squares minimization that impels the final estimates to be equal to the pre-smoothing estimation. The second term, $\left\|L \tau_{D}\right\|_{2}^{2}$, minimizes the surface Laplacian of the estimates. This is obtained through multiplication of $\tau_{D}$ by a numerical approximation of the Laplace operator $L$ obtained as in [130].

The trade-off between these two terms is set by the parameter $\gamma$. For small values of $\gamma$, the final time estimates are similar to the noisy estimation, while for large values of $\gamma$ the resulting time estimates tend to zero. The choice of this smoothing parameter is determined by the user and depends on the final application. We explored the effect of different degrees of smoothing for estimation of activation and recovery times and beat localization. We analyzed the results without applying any smoothing $(\gamma=0)$, applying a limited $(\gamma=200)$ and an extreme amount of smoothing $(\gamma=200,000)$.

\subsubsection{RESUlts AND DISCUSSION}

Figure 7.5 shows the results. Activation-time estimation is most accurate when the spatiotemporal method is employed. Regardless of the estimation method, there is a clear 

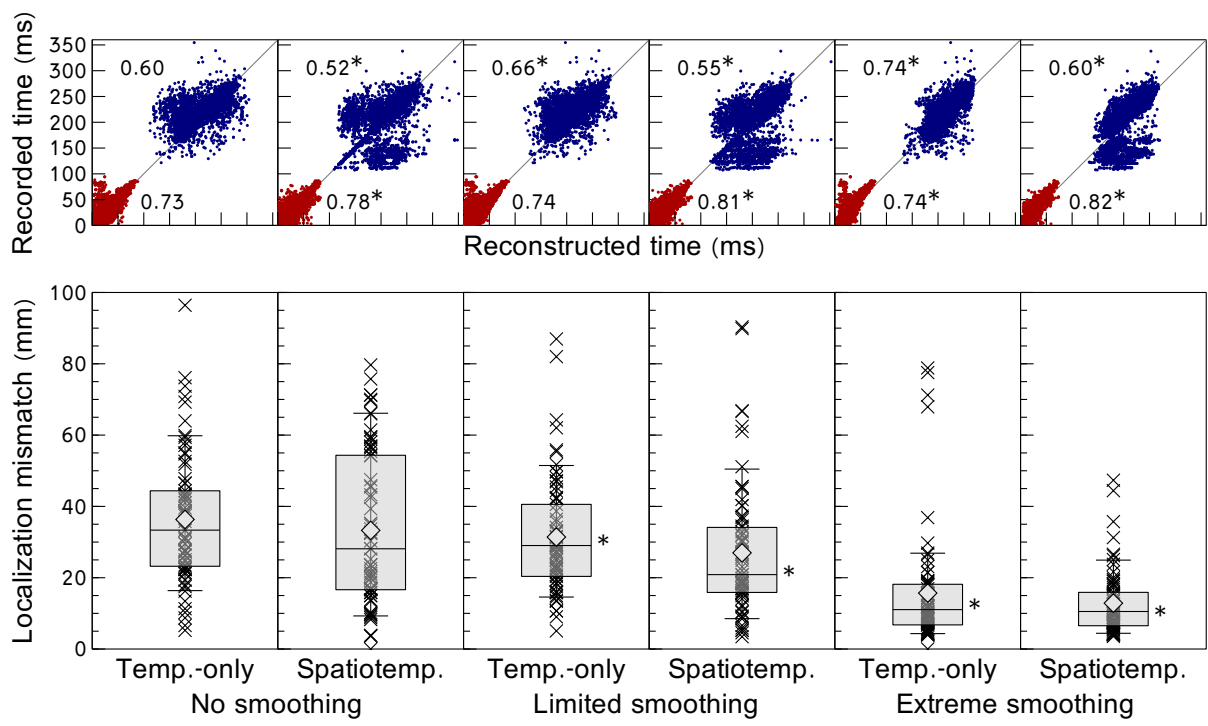

Figure 7.5: In vivo accuracy of the temporal-only ('Temp.-only') and spatiotemporal ('Spatiotemp.') approach to activation/recovery-time estimation, with no (left two columns), limited (middle two columns), and extreme post-estimation spatial smoothing (right two columns). Top row: activation (red) and recovery times (blue) as determined from the recorded epicardial electrograms (vertical axis) and reconstructed epicardial electrograms (horizontal axis); R-values of the linear fit are shown. Bottom row: distance between known origin of pacing and reconstructed location of earliest activation. Box spans the 25-75\% range; whiskers at $9-91 \%$ range; median indicated by horizontal line; mean by diamond. Asterisk $(*)$ indicates statistically significant difference from the temporal-only-no-smoothing results.

added benefit of additional spatial smoothing. There is small difference between limited or extreme smoothing in terms of activation time accuracy. These results show that incorporating spatial information (either by the spatiotemporal approach or by spatial smoothing) is beneficial for the estimation of activation times.

As a consequence of more accurate activation times, localization of beat origins (defined as the location of earliest activation) is improved as well. In our canine validation experiments, the median accuracy of temporal-only beat localization was only $33 \mathrm{~mm}$. Spatiotemporal localization of beat origins had a median accuracy of $25 \mathrm{~mm}$, which improved further to $20 \mathrm{~mm}$ with limited additional spatial smoothing, and to $10 \mathrm{~mm}$ with extreme smoothing. Additionally, localization variability was reduced strikingly, especially with extreme additional spatial smoothing. The large effect of additional spatial smoothing can be explained by the fact that beat origins are determined by the single epicardial node with earliest reconstructed activation time; outliers with incorrect early 
activation times will therefore have a large impact. Additional spatial smoothing removes these sporadic outliers and increases the chance of detecting the true location with earliest activation.

Interestingly, recovery-time estimation did not improve with the spatiotemporal approach. This could be explained by the more gradual pattern of recovery, which results in less pronounced spatial gradients. Therefore, there is no added benefit of including a spatial gradient when estimating the upslope of the $\mathrm{T}$ wave, even the opposite: the spatiotemporal approach decreased accuracy of recovery-time estimation. Regardless of the estimation method, there was added benefit from additional spatial smoothing.

In conclusion, accuracy of activation times determined from reconstructed epicardial electrograms is improved when a spatiotemporal approach is used. Recovery times are best determined with a temporal-only approach. Additional spatial smoothing benefits both activation- and recovery-time estimation, and yields the results presented in Chapter 3 .

\subsection{The MINIMUM NUMber OF BODY-SURFACE EleCtrodeS}

Since the development of the ECG, it has been investigated how many electrodes are needed to reliably diagnose or localize cardiac electrical events, and which electrode positions are most important. Also for ECGI, the minimum number of body-surface electrodes remains a subject of discussion (and their optimal positioning), but is usually taken to be far more than the nine electrodes of the 12-lead ECG. Currently-used setups include 64 [41], 120 [35] or 256 [45] electrodes. Recently, the use of only the standard 12-lead ECG was shown to be worthwhile in some cases in a different implementation of ECGI, and work is ongoing to validate the use of such a small number of body-surface electrodes for beat origin localization. [53], [163]

In this section, we will investigate how many electrodes are needed for accurate epicardial reconstructions in our implementation of ECGI. More precisely, for reasons of practicality, we have investigated what the optimal number of electrode strips is. Each electrode strip contains 8 or 12 electrodes. Reducing the number of electrode strips can help to reduce the time investment needed for preparation and cleaning of the electrodes. We evaluated this in one dog of the canine experiments of Chapter 3. In that dog, 192 body-surface electrodes were used and included in the transfer matrix that describes the relation between body-surface potentials and heart-surface potentials. Of all implanted epicardial electrodes, 52-73 electrodes recorded a good signal (number of well-connected electrodes could change per beat). Those reference electrograms were 


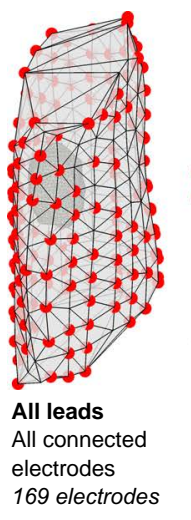

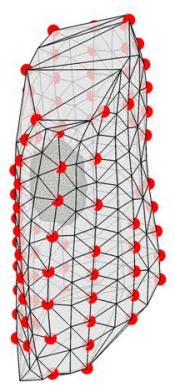

Half 1

Half of electrode columns

108 electrodes

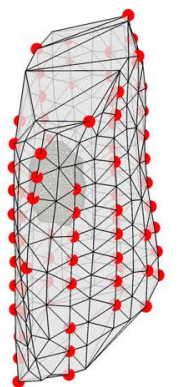

Half 2

Other half of columns 80 electrodes

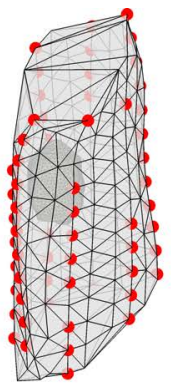

Third 1

$1 / 3$ rd of electrode Other $1 / 3$ rd of

columns columns

80 electrodes $\quad 68$ electrodes

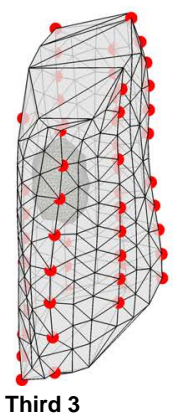

Third 3

Remaining $1 / 3 \mathrm{rd}$
of columns

59 electrodes

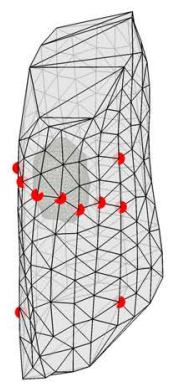

10 electrodes

Default 12-lead

electrodes $+\mathrm{RL}$

10 electrodes

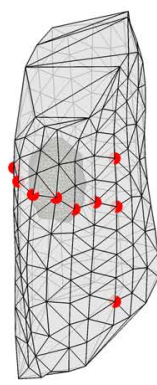

9 electrodes

Default 12-lead

electrodes

9 electrodes

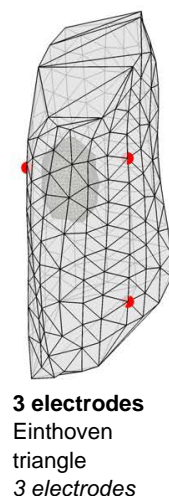

triangle

3 electrodes

Figure 7.6: The full and reduced-lead setups used for noninvasive reconstruction of epicardial potentials and activation locations. The transparent mesh represents the body surface, and the heart surface is shown as well. The red dots in the full-lead setup represent all bodysurface electrodes with a good recording quality. In the subsequent setups, only subsets of these electrodes are used.

compared to the reconstructed electrograms from the corresponding (closest) virtual epicardial node. Selected epicardial electrodes were also used for pacing.

Of the 192 body-surface electrodes, 169 electrodes had a connection to the skin that provided electrograms of high quality. This full set of well-connected electrodes was compared with reduced-lead sets, see Figure 7.6. Reduced-lead sets were created by selecting only part of the electrode strips (visible as columns in the geometry). For example, the set 'Half 1 ' was based on selecting every other strip of electrodes; the set 'Half 2' was formed by its complement. Due to an uneven number of strips, one strip was included in both sets, resulting in a combined number of electrodes that exceeds the initial set. In a similar way, three sets of leads with $1 / 3$ of the electrode strips were created. Electrode sets used clinically were also evaluated. The default 12-lead electrocardiogram 


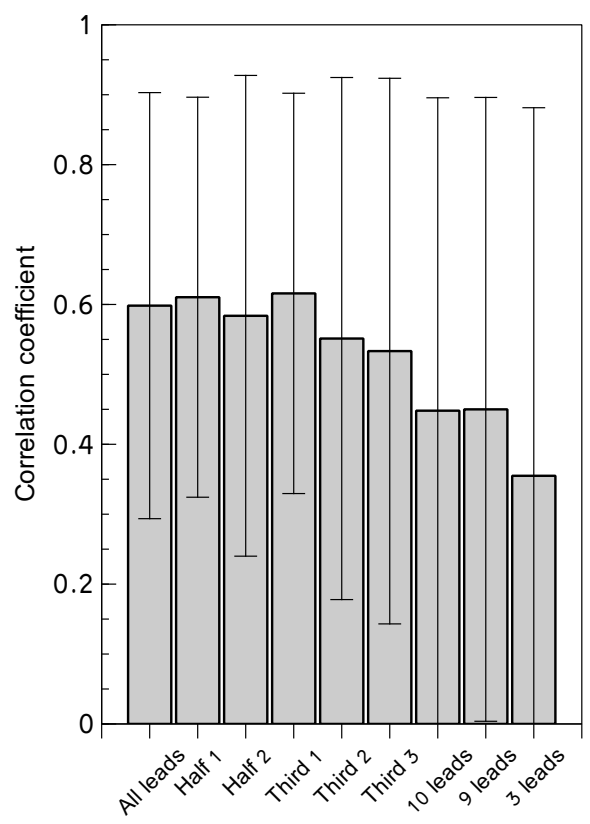

(a)

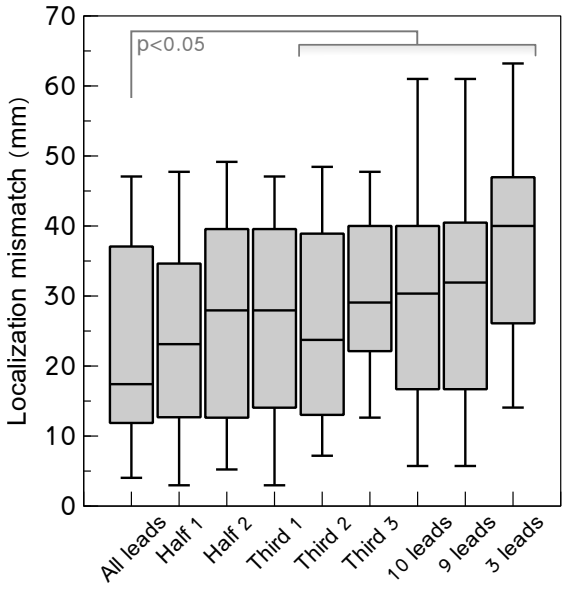

(b)

Figure 7.7: Accuracy of reduced lead-setups in ECGI. a) Correlation coefficients between reconstructed and recorded electrograms, averaged over all epicardial electrodes in all 39 beats in each lead setup. b) Box plots of the mismatch between reconstructed and known locations of earlieast activation, for all 27 paced beats in each lead setup. Horizontal line in the box indicates the median; the box spans the range bewteen the first and third quartiles.

is based on 9 electrodes, forming the ' 9 -electrodes' set. In one set, an additional electrode on the right leg was included, resulting in the '10-electrodes' set. Finally, a '3-electrodes' set was formed by Einthoven's triangle.

Figure 7.7a shows the correlation coefficients for all 52-73 epicardial electrodes for all 39 beats in one dog, averaged per lead-setup. This shows that the quality of the reconstructed electrograms remains stable from 169 (set 'All leads') to 59 (set 'Third 3') electrodes, and then decreases with a lower number of electrodes. Similarly, the mismatch between the detected origin of a beat and known pacing location decreases when fewer body-surface electrodes are used, see Figure 7.7b. However, it is striking that when only 9 or 10 electrodes are available for pacing localization, the median mismatch is only marginally higher than when half of the electrodes are used. This might be the result of the spatiotemporal approach when determining the activation times, in which neighboring nodes on the virtual epicardial surface are taken into account when determining 
the activation times. This procedure also involves smoothing, which might be beneficial when only a few electrodes are used to reconstruct electrograms on thousands of epicardial nodes.

In this study, we have not investigated the effect of including a more detailed bodysurface geometry in the transfer matrix. In all lead-setups, the transfer matrix was computed on the original 192 body-surface electrodes. A more detailed body-surface geometry might improve the reconstruction quality in reduced-lead setups even further.

Additionally, the regularization parameter was determined on the full set of electrodes. Selecting the regularization parameter per reduced-lead setup might be beneficial (selecting a more appropriate balance between the available information from the limited number of electrodes, and the need for regularization), but could also be disadvantageous (as simply less information is available to determine that balance).

Moreover, we have not investigated optimal positioning of the electrodes. One might expect that the anterior side of the thorax may contain more relevant information than the posterior or lateral sides. However, this could differ between beat types.

In conclusion, our study showed that a minimum of 59 electrodes is needed for high quality reconstructions of electrograms. To take into account disconnection of electrodes, a minimum of 80 electrodes seems advisable. For specific purposes (such as detecting the origin of an extrasystolic beat), a lower number of body-surface electrodes could provide results that are still useful. However, especially when using only a 12-lead setup, it is important to realize that although the median results are reasonable, there is a significant spread of the error, up to a $65 \mathrm{~mm}$ mismatch.

\subsection{THE ADDED BENEFIT OF SPARSE DATA REPRESENTATION}

The work in this thesis was based on the potential-based source model, i.e., our implementation of ECGI reconstructs potentials at the epicardium. As explained in Chapter 1 , this is only one of possible source models. The alternative EDL source model directly reconstructs activation and recovery times at the heart surface. It has the (at least theoretical) advantage that this source description is simpler than the potential-based model: where the EDL model has two parameters per node per beat (time of activation, and time of recovery), the potential-based model has one parameter per time instant per beat (electrical potential). For a beat of $500 \mathrm{~ms}$ recorded at $2048 \mathrm{~Hz}$, this means $\sim 1000$ values to be estimated per node for the potential-based model, as opposed to two for the EDL model.

Although the simplicity of the EDL model might be an advantage when dealing with ill-posedness, the lack of electrograms prevents investigation of abnormalities such as low 
amplitude potentials, fractionated electrograms, local ST elevation etc. Moreover, the EDL model requires assumptions regarding activation and recovery that may not hold in pathological circumstances. For example, the shortest-path algorithm used as a propagation model in the Oosterom - Van Dam implementation may not apply in scarred tissue. [164] Additionally, these investigators assume an equal conduction velocity for activation and recovery [164], while we have shown that recovery patterns in animals and humans can be very different from activation patterns (and thus have different velocities). Therefore, the potential-based source model, which makes less restrictive assumptions on cardiac electrophysiology, could provide more accurate results, especially in pathological circumstances.

In this thesis we have not only investigated the potential-based source model, but we have also developed two source models that are derived from it. By using a different representation of the data in the reconstruction process, a lower complexity of the source model is achieved. Both source models use the novel data representations only as intermediates, and can thus easily be converted to potentials and electrograms. Therefore, although the end result is epicardial potentials, the actual source models are based on different models: 'physiologically likely potential patterns' in physiology-based regularization (PBR, Chapter 4) and 'wavelet coefficients' in wavelet-based regularization (WBR, Chapter 5 ).

\subsubsection{COMPLEXITY OF THE NOVEL SOURCE MODELS}

For the potential-based model, the number of parameters that is reconstructed per time instant is equal to the number of virtual epicardial nodes, typically around 1,500. For a beat of $500 \mathrm{~ms}$ recorded at $2048 \mathrm{~Hz}(\sim 1000$ time instants), the total number of parameters for a beat equals $1,500 \times 1,000=1,500,000$.

In PBR, the number of parameters estimated per time instant is equal to the size of the novel basis, and does not depend on the number of virtual epicardial nodes. In our results, a basis size of 10 was optimal. For a beat of $500 \mathrm{~ms}$ at $2048 \mathrm{~Hz}$, this results in $1,000 \times 10=10,000$ parameters, significantly less than the direct potential-based model.

In WBR, the number of parameters is actually higher, as the potentials per virtual node are decomposed. The increase in number of parameters depends on the number of levels in the wavelet decomposition, and the type of decomposition (stationary or discrete). For a stationary wavelet decomposition on three levels, the number of parameters for a full beat is 4 ( 1 approximation +3 detail levels) $\times 1,000$ (time instants) $\times 1,500$ (virtual epicardial nodes) $=6,000,000$. 
A

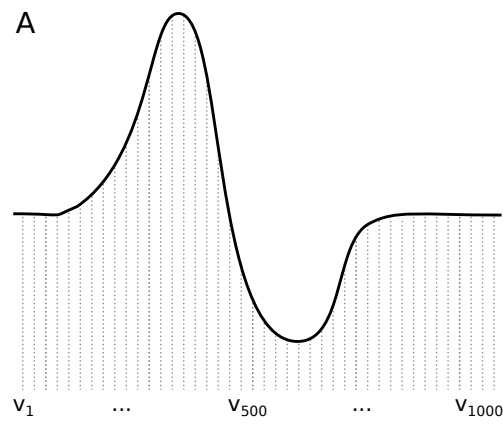

B

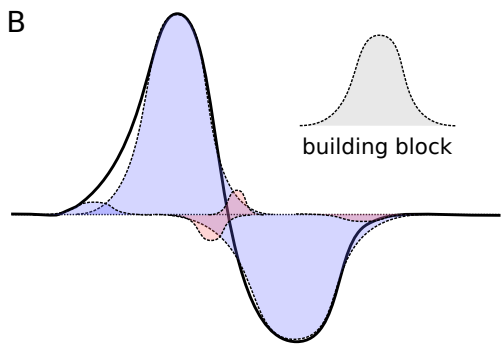

Figure 7.8: The role of building blocks in efficient (sparse) data representation. Panel A: normal data representation of a time signal, such as an electrocardiogram. The signal is described by a value $v_{i}$ at each time $i$, resulting in 1,000 values for a single electrocardiogram. Panel B: a building block that closely resembles the expected data allows to specify a signal with only a few parameters, in this case the position, width and height of the building blocks. All other parameters (i.e, of the unused positions, widths and heights) can be set to zero or ignored all together, resulting in a sparse description of the data. Building blocks can be used both with an additive (blue) and subtractive (red) purpose.

Thus, the model complexity of PBR is inherently lower than that of the normal potential-based formulation, whereas that of WBR is higher. However, by pursuing a sparse representation, many of the parameters of PBR and especially WBR are set to zero and ignored. Effectively, this reduces model complexity (further).

\subsubsection{SPARSity OF DATA REPRESENTATION}

Sparsity means that only relatively few parameters are needed to describe the data, and the others can be ignored (i.e., set to zero). For this, it is essential that the data representation closely resembles the true source. A good choice for the data representation is thus essential, as illustrated in Figure 7.8. In the PBR method, this is achieved by simulating potential patterns with realistic propagation methods. These potential patterns are then used as basis (or building blocks) for the reconstruction. As the building blocks already closely resemble the expected source data, only few building blocks are necessary to capture this. In the WBR method, the building blocks are formed by a so-called mother wavelet, which can be shifted in time and amplitude to match the source data. We chose the Daubechies 2 wavelet, a common choice for cardiac signals [126], and only a few parameters would be necessary to describe real epicardial electrograms in terms of this mother wavelet. We also designed a mother wavelet based on epicardial electrograms, and obtained similar results. Different methods are available to estimate these parame- 
ters while enforcing sparsity, i.e. estimating the parameters such, that most are zero and only a few are non-zero. [165]

We have shown, for both the PBR and WBR methods, that such data representations in combination with algorithms that enforce sparsity indeed provide reconstructions of epicardial electrograms and beat origins that are more accurate than the traditional zeroth-order Tikhonov method used in Chapter 3.

\subsubsection{IMPLICATIONS FOR CLINICAL APPLICATIONS}

The freedom in data representation might be exploited further for specific clinical goals. For example, if a patient has myocardial scar tissue (e.g., detected with MRI), this area of abnormal conductivity could be included in the potential patterns that form a basis in the PBR method. This could improve reconstruction quality in this specific patient. In the WBR method, temporal characteristics of electrograms (e.g., fractionation for myocardial scar, or $\mathrm{Q}$ morphology for beat origin) could be used as mother wavelet to improve their reconstruction accuracy. Thus, the choice of data representation could be useful to improve reconstruction accuracy for specific clinical questions. We have shown that this is true when information about the origin of the beat (left versus right) is included in the reconstruction basis (see Chapter 4), resulting in higher accuracy of reconstructed electrograms. Of course, the choice in data representation can also reduce reconstruction quality if the assumptions (e.g., origin of a beat) do not fit with reality.

\subsection{CONCLUSions ANd Future Work (PARt I)}

The main insights into ECGI provided by this thesis are the following:

- Electrogram reconstruction is accurate but shows considerable variability

- Spatial mismatch between recorded and reconstructed electrograms is a main cause for variable accuracy of reconstructed electrograms

- Spatial resolution between recorded electrograms and highly correlating reconstructed electrograms is $<2 \mathrm{~cm}$

- Beat origin localization is accurate $(\sim 1 \mathrm{~cm})$ and highly reproducible

- Electrogram variability is well addressed by using a spatiotemporal approach when estimating activation and recovery times

- Cardiac mechanics should be incorporated in ECGI

- Accuracy of ECGI is independent of the number of body-surface electrodes if more than 80 electrodes are used

- Sparsity can help to improve reconstruction quality, especially for specific goals 


\section{Part II: Novel insights obtained with ECGI}

In this part of the discussion, we introduce and discuss some of the clinical and scientific applications for which we have used ECGI: guiding ablation in the cardiac electrophysiology (EP) lab, integrating imaging modalities and ECGI, and investigating the role of recovery abnormalities in arrhythmia mechanisms.

\subsection{PVC ABLATION IN THE EP LAB}

PVCs are ectopic beats from the ventricles. They are the result of focal depolarization in a discrete region of ventricular myocardium [166], due to enhanced automaticity, triggered activity, or reentry [167] (see also Chapter 1 , Section 1.2). PVCs can occur in the absence of structural heart disease, or develop in relation to ischemic myocardial disease, valvular heart disease, and cardiomyopathies. [166] PVCs are found in the majority of the population to some extent, with $1 \%$ prevalence per single ECG and a little over $50 \%$ prevalence in a 24-48 hour Holter recording. [167] However, not the presence of PVCs, but their frequency (PVC burden) is associated with comorbidities and decreased left ventricular ejection fraction (LVEF), a measure for cardiac pump function. [166] Prospective studies have suggested that frequent PVCs may not only be associated with the presence of a cardiomyopathy and reduced LVEF, but may have a direct detrimental effect on LVEF. [166], [168].

\subsubsection{INCREASED RISK FOR SUDDEN CARDIAC DEATH}

PVCs are also associated with an increased risk for sudden cardiac death (SCD) in some populations. [167] Especially short-coupled PVCs have been described as potential triggers of VF [167], amongst others in Brugada syndrome [169], early repolarization syndrome [170], idiopathic VF [171] and after myocardial infarction [172], but also in the absence of apparent heart disease [173], see also Chapter 6. No cutoff value for a tight coupling interval has been defined, although most often it was $<400 \mathrm{~ms}$ under these circumstances. [167]

It is important to realize that heart disease, although not obviously present, can be associated with frequent PVCs. For example, arrhythmogenic right ventricular cardiomyopathy (ARVC), catecholaminergic polymorphic ventricular tachycardia, and $\mathrm{SCN}_{5} \mathrm{~A}$ mutations are often associated with frequent PVCs. A family history of SCD should prompt to look for these and other disease characteristics. However, neither the presence of frequent PVCs nor an identified cardiomyopathy are required for malignant arrhythmias to develop. [167] 
One large prospective study investigated the association between the prevalence of PVCs and risk for SCD in the general population, in the absence of apparent heart disease. [174] In that study, with 15,00o apparently healthy white males, 2 minutes of ECG recordings revealed a prevalence of PVCs of $4.4 \%$. The subgroup of participants with PVCs had an increased relative risk for SCD $(\mathrm{RR}=3.0)$, but no increased risk for noncardiac sudden death. Higher PVC burden and complexity (multiforms, pairs, runs, Ron-T) were associated with a higher relative risk, especially in participants younger than 50 years. [174]

\subsubsection{Treatment Of FreQuent PVCs}

Treatment of PVCs can therefore be necessary and depends on symptoms and LV dysfunction. Symptoms can include palpitations, lightheadedness, dyspnea, chest discomfort and exercise intolerance. However, frequent PVCs are often asymptomatic. High burden of ventricular ectopy ( $>20 \%$ per 24 hour Holter monitoring) is associated with LV dysfunction and can require PVC therapy independent of symptoms. [166] Therapy to suppress PVCs includes drugs and catheter-based ablation. In general, higher PVC burden, unifocal PVC morphology, presumed endocardial origin, and PVC-mediated LV dysfunction may favor catheter ablation, while pleomorphic PVCs and severe comorbidities favor pharmacological therapy. [167] Initial drug therapy includes betablockers or calcium channel blockers and may be followed by therapy with (other) antiarrhythmic drugs. [166], [167] Ablation therapy is a safe and effective option for patients with symptomatic frequent PVCs and in those with suspected PVC-induced or PVCaggravated cardiomyopathy and can be a therapy of first choice. [166] In a randomized controlled study, ablation of PVCs successfully normalized LV function in $82 \%$ of the patients. [168] Additionally, ablation therapy should be considered as a first-line therapy in patients in whom PVCs are identified to trigger VF. [167] In a recent review, therapy choices for patients with frequent PVCs are summarized as follows: [166]

- In the absence of both symptoms and structural heart disease, observation is appropriate;

- In patients with symptoms but no structural heart disease, medical therapy is appropriate as first line therapy;

- And in the patient with structural heart disease with or without symptoms, catheter ablation is appropriate as first choice.

Catheter ablation is based on electrically silencing the tissue that is triggering the PVC. After introduction of the catheter in the heart, a combination of activation mapping and pace mapping is used to determine the PVC origin. [167] Activation mapping is 
performed by positioning the catheter on the myocardial tissue and recording the local electrogram during a PVC. By iteratively repositioning the catheter, the location with earliest activation time (EAT) is identified as the origin of the PVC. This procedure is usually performed point-by-point and thus beat-by-beat, and can be very time consuming if the patient does not have frequent PVCs. The occurrence and frequency of PVCs are often reduced during the procedure due to use of sedatives or anesthetics.

Pace mapping is based on giving a local electrical stimulus with the catheter electrode, activating the myocardial tissue from that location. The 12-lead ECG of the resulting beat is then compared to the (preprocedurally recorded) ECG of the clinically relevant PVC. The correspondence between the paced beat and PVC can be expressed as number of leads with high correlation (e.g., a 11/12 pacemap has a good correspondence in 11 leads of the 12 -lead ECG), or as averaged correlation coefficient over all leads (e.g., $97 \%$ correlation). Although pace mapping is less accurate than activation mapping, it may be the only possibility for localizing PVC origins if PVC frequency is low.

\subsubsection{ROLE OF ECGI IN ABLATION THERAPY}

Alternatively, ECGI could be used to reconstruct PVC origins before the EP procedure. It reconstructs activation times per beat and can thus localize the origin of a PVC based on a single recorded beat. Moreover, body-surface potential recording for ECGI can be performed up to several hours, significantly increasing the likelihood of capturing a clinically relevant PVC. Additionally, ECGI can be performed without sedatives and anesthetics, which are known to suppress PVCs. When ECGI is combined with threedimensional mapping systems, ECGI's preprocedural noninvasive PVC activation map can be merged with the invasively recorded anatomy during the ablation procecure. This allows the cardiologist-electrophysiologist to see the position of the catheter live during the procedure within the ECGI map. The noninvasive PVC activation map can then be used to guide the ablation.

We have performed ECGI for PVC ablation therapy in several cases. One case is illustrated in Figure 7.9. This female of 67 years old presented with relatively limited, but very symptomatic PVCs (Holter 24 hour recording: 1,347 PVCs). The PVCs were monomorphic, with a suspected origin in the $\mathrm{LV}$, and were also recorded during the BSP recording for ECGI, see Figure 7.9, top. The ECGI-reconstructed activation map of the PVC showed a suspected origin on the inferior midbasal LV-RV groove.

The patient had no spontaneous clinically-relevant PVCs during the ablation procedure. Beta-adrenergic stimulation with isoproterenol did not produce any PVCs. Based 

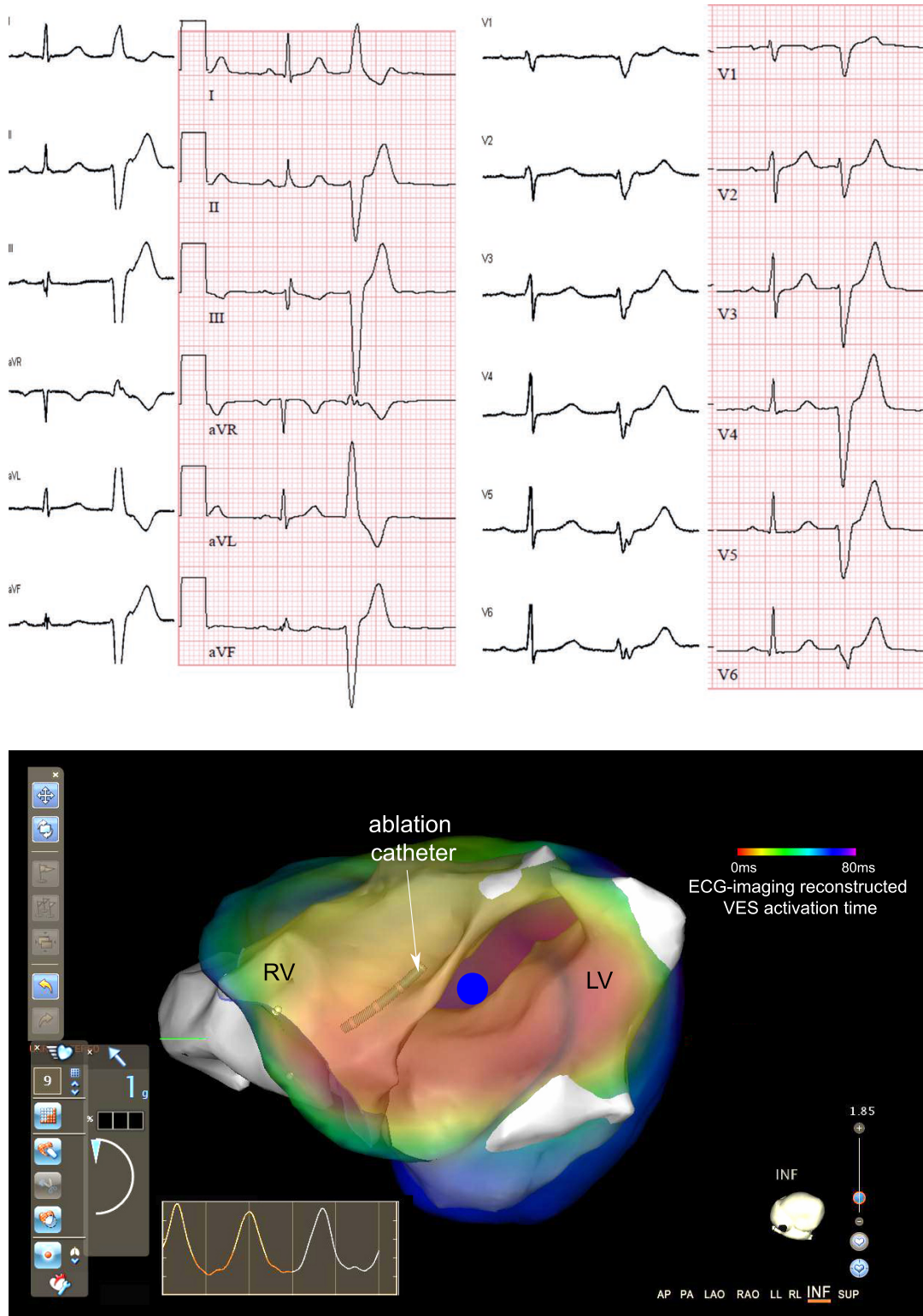

Figure 7.9: First ECGl-guided ablation case in MUMC+. Top: 12-lead ECG recorded during ECGI (white background) and clinical recording during symptoms (pink background), both with a sinus beat and subsequent PVC. Bottom: Live view as visible to the cardiologistelectrophysiologist during the ablation procedure when using the Carto 3 cardiac mapping system. The 3D anatomy as determined by the Carto catheter (white structures) is overlaid with the activation map of the PVC beat as created preprocedurally with ECGI (red-to-blue: early-to-late activation time; blue dot: PVC origin). The cardiologist can use this live view during the procedure to navigate the catheter to the area suspected of the PVC origin. 
on the ECGI prediction, endocardial pace mapping was performed on the LV and RV midbasal sides of the septum and resulted in a 6/12 and 12/12 score, respectively. Radiofrequency ablation was performed on two RV locations with 12/12 pacemap and resulted in runs of PVCs with the clinically suspect morphology. The ablation locations were 25 and $25 \mathrm{~mm}$ from the predicted PVC origins. Although the ablation was performed endocardially, ECGI can only project PVC origins on the epicardium. Thus, the distance between the ablation location and predicted PVC origins includes the thickness of the myocardial wall (in this patient $5 \mathrm{~mm}$, normally for a healthy RV $<5 \mathrm{~mm}[175]$ ), the intracardiac distance to the septum and potentially any deviating activation pattern.

At three-month follow up, the patient experienced a substantial subjective improvement. There was an almost complete absence of PVCs on a 24-hour Holter recording, with just 8 PVCs recorded. Thus, ECGI facilitated the ablation procedure, which led to a successful reduction of extrasystolic activity and reduced subjective burden.

The initial results of PVC ablations of several cases in MUMC+ are shown in Table 7.1. This table shows that in general, the mismatch between noninvasive ECGI prediction and invasive therapy was around $19 \mathrm{~mm}$. Assuming that the PVC originated from the location that was ultimately ablated, this could be interpreted as the mismatch of ECGI-based PVC localization. This mismatch is higher than the pacing mismatch that was determined in the canine validation experiments, where a median mismatch of 10 $\mathrm{mm}$ was found (Chapter 3 ). Reasons for this increased mismatch include: the thickness of myocardial wall in case of endocardial ablations, whereas the ECGI prediction is always projected on the epicardium; or the distance from the epicardium to the septum in case of septal ablations; a mismatch between the fusion of the three-dimensional mapping system (which included the exact position of the ablation locations) and the CT scan (on which ECGI predictions were based); the difference in body position during ECGI (semi-supine during body-surface potential mapping, supine during CT) and the ablation procedure (supine); and, although unlikely with monomorphic PVCs, a mismatch in the reconstructed PVC (recorded weeks to months before the ablation) and the ablated PVC.

To guide therapy, a $2 \mathrm{~cm}$ accuracy may be sufficient. During ablation therapy, the location of ablation is confirmed with invasive activation and pace mapping. This may be greatly sped up by reducing the initial mapping area to an area with a $2 \mathrm{~cm}$ radius with ECGI. However, for completely noninvasive treatment, as suggested recently by $\mathrm{Cu}$ culich et al [176], a more precise localization might be necessary. This can be achieved by addressing the issues mentioned in the previous paragraph; additionally, PVC localization could become more accurate when using not earliest activation time, but location of deepest negative potential. [103] 


$$
1 \text { I }
$$

\begin{tabular}{|c|c|c|c|c|c|c|c|c|c|c|c|}
\hline$Y_{5}$ & 1 & PVCs & No & Pre & Endo & $\begin{array}{l}\text { Basal posterior lateral } \\
\text { free wall of } L V\end{array}$ & $12 / 12$ & 11,227 & 24,441 & $\begin{array}{l}\text { Basal superior lateral } \\
\text { free wall of } L V\end{array}$ & $30^{*}$ \\
\hline \multirow[t]{2}{*}{$\mathrm{B}_{3}$} & 2 & PVCs & No & Live & Endo & $\begin{array}{l}\text { Inferior } \\
\text { midbasal RV }\end{array}$ & $12 / 12$ & 1,347 & 8 & $\begin{array}{l}\text { Inferior midbasal } \\
\mathrm{RV} / \mathrm{LV} \text { groove }\end{array}$ & 25 \\
\hline & 3 & & & & Endo & $\begin{array}{l}\text { Inferior } \\
\text { midbasal RV }\end{array}$ & $12 / 12$ & 1,347 & 8 & $\begin{array}{l}\text { Inferior midbasal } \\
\mathrm{RV} / \mathrm{LV} \text { groove }\end{array}$ & 25 \\
\hline $\mathrm{U}_{9}$ & 4 & PVCs & No & Pre & Endo & $\begin{array}{l}\text { Inferior } \\
\text { basal LV }\end{array}$ & & 40,000 & 450 & $\begin{array}{l}\text { Inferior basal } \\
\mathrm{RV} / \mathrm{LV} \text { groove }\end{array}$ & 20 \\
\hline
\end{tabular}

\begin{tabular}{|c|c|c|c|c|c|c|c|c|c|c|}
\hline & 5 & & & & & & & & & 24 \\
\hline \multirow[t]{2}{*}{$\mathrm{H}_{2}$} & 6 & PVCs, VT & ARVC & Live & Endo & $\mathrm{RV}$ free wall & $97 \%$ & 4,621 & $\mathrm{RV}$ free wall & 21 \\
\hline & 7 & & & & Epi & Septal RVOT & $91 \%$ & & RVOT & 3 \\
\hline $\mathrm{N}_{9}$ & 8 & PVCs & Scar & Pre & Endo & Inferior basal RV & $97 \%$ & 14,000 & $\begin{array}{l}\text { Inferior midbasal } \\
\mathrm{RV} / \mathrm{LV} \text { groove }\end{array}$ & 19 \\
\hline $\mathrm{F}_{2}$ & 9 & PVCs & No & Live & Endo & $\begin{array}{l}\text { Inferior RV } \\
\text { free wall }\end{array}$ & $90 \%$ & 2,300 & $\begin{array}{l}\text { Inferior RV } \\
\text { free wall }\end{array}$ & 20 \\
\hline \multirow[t]{3}{*}{ H6 } & 10 & PVCs & No & Live & Endo & Septal RVOT & $11 / 12$ & 23,000 & Septal RVOT/aorta & 10 \\
\hline & 11 & & & & Endo & Septal aorta $†$ & $12 / 12$ & & Septal RVOT/aorta & 7 \\
\hline & & & & & & & & & Average distance & 19 \\
\hline
\end{tabular}

Table 7.1: PVC ablations guided by ECGI in MUMC+. Note that the distance between ECGI-predicted PVC origin (projected on the epicardium) and ablation location can include the thickness of the myocardial wall in case of endocardial ablation. Pre: ECGI was performed preprocedurally to guide ablation. Live: ECGI was performed preprocedurally and live during the ablation. Endo: endocardial. Epi: epicardial. *: Distance estimated visually as no image fusion was possible at this stage. $\dagger$ : Not ablated due to close proximity to left main stem coronary artery. 


\subsubsection{Use of ECGI in PVC AbLATION OUTSIDE MUMC+}

Although these were the first ECGI-guided ablation cases in Maastricht, similar setups have been applied previously at other hospitals. The commercially available ECVUE system (CardioInsight, Cleveland, $\mathrm{OH}$ ) was used to localize PVC origins preprocedurally and was shown to be superior in PVC localization compared to the 12-lead ECG. [177] It resulted in fewer RF applications required to terminate PVCs, decreased time to first RF application, and decreased total procedure time. However, it did increase patient radiation exposure due to the need for a CT scan. [177]

In a recent prospective randomized study with 42 participants, patients were divided in a group that received ablation based on trigger-localization with ECVUE, and a group that was ablated based on 12-lead ECG localization. ECVUE-based ECGI was shown to be able to localize PVCs better than the 12-lead ECG. [178] The ventricle of origin (LV vs RV) was identified correctly in $95.2 \%$ of the cases with ECVUE, and only $76.6 \%$ with 12-lead ECG. Sublocalization within the ventricles was accurate in $95.2 \%$ of the cases with ECVUE, and $38.1 \%$ with 12 -lead ECG. As a result, the number of radiofrequencyenergy applications was significantly lower when using ECVUE-based localization ( 2 applications) than when using 12 -lead localization (4 applications), and procedural times were shorter. [178] Ablation success was similar in both groups, both acutely and at three-month follow up. Failure of ECVUE to localize PVCs mainly occurred in PVCs that originated near the septum, especially when localized at the LVOT or RVOT. [177]

In a study using NEEES (EP Solutions SA, Yverdon-les-Bains, Switzerland), PVCs were localized in 20 patients. In $86 \%$ of the cases, the correct ventricular segment was diagnosed. [179] These studies confirm that ECGI can contribute significantly to ablation therapy.

Methods are being developed that localize PVCs based on the wave-front-based implementation of ECGI. [53], [180] Although it is based on the 12-lead ECG only, it includes a patient-specific geometry and inverse reconstruction. In a preliminary study with 9 participants, this system was able to successfully localize PVCs (defined as location of successful ablation) in $86 \%$ of the cases, whereas human-based 12-lead ECG localization of PVC had a 27\% accuracy. [163] This confirms our results from Section 7.3, where we demonstrated that although electrogram correlation decreases significantly, beat localization with ECGI was reasonable accurate with a limited number of electrodes. 


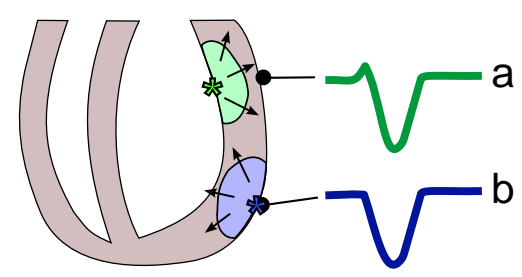

Figure 7.10: Illustration of the genesis of epicardial electrograms for epicardial or endocardial origins of a PVC. The epicardial electrogram at the location of epicardial breakthrough is expected to yield an $\mathrm{rS}$ morphology for an endocardial origin (a), as the initially approaching wave front will give rise to a (small) positive deflection, and the subsequently passing wave front a negative deflection. An epicardial origin (b) will only yield a negative deflection, resulting in a $\mathrm{Q}$ morphology on the local epicardial electrogram.

\subsubsection{ENDOCARDIAL VS EPICARDIAL ORIGINS}

Another valuable aspect of PVC localization would be to distinguish between an endocardial or epicardial origin. In the clinic, this could facilitate the choice between an endocardial or transthoracic epicardial catheter approach, reducing the need to convert from one to the other in specific cases. As we have demonstrated in Figure 3.7 in Chapter 3, an endocardial origin gave an $\mathrm{rS}$ morphology on the epicardial electrogram, and an epicardial origin yielded a Q morphology, see also Figure 7.10. However, at the same time, we have shown that there is a significant variability in the accuracy of a single electrogram. For example, epicardial electrogram 5 in Figure 3.2B exhibited an rS morphology in recording, but missed this initial $r$ peak in reconstruction, showing a $\operatorname{Qr}$ pattern. These morphological differences could be clinically relevant when determining the origin of activation.

Others have investigated this aspect as well. The authors of the ECVUE based study reviewed local unipolar electrogram morphology at the earliest epicardial breakthough, to differentiate between endocardial (rS morphology) and epicardial (Qmorphology) origin; however, they did not provide evidence for accurate differentiation. [177] The authors of the wave-front-based approach suggested that their method is able to differentiate between endo-, epi- and mid-myocardial origins. [163]

In other words, although local electrogram morphology may be suggestive for an endocardial or epicardial origin, currently no convincing prospective results are available to answer this clinically very relevant question.

\subsubsection{REAL-TIME ECGI}

With advancements in computational power, real-time application of ECGI becomes possible. This requires obtaining and processing CT or MRI data in a short amount of 
time, while the body-surface electrodes are attached, in order to have the transfer matrix available for inverse reconstruction of epicardial potentials as soon as the body-surface potential recordings are started. Additionally, real-time application of ECGI requires efficient algorithms to detect and handle disconnection of electrodes, to adjust regularization parameters to changes in recording quality, to determine activation and recovery isochrones from reconstructed electrograms, and to visualize the requested data. First steps to achieve this are made by commercial, easy-to-use packages such as those from CardioInsight (Cleveland, OH, USA).

Real-time application in the EP lab could help to support PVC and VT ablation therapy by investigating and localizing arrhythmogenic effects of drugs or burst pacing. In addition, dynamic changes in electrical substrate (e.g., steep gradients of repolarization) could be investigated directly, and effects of drug therapy evaluated. Thus, real-time ECGI could help to provide better understanding and possibly treatment of arrhythmias.

\subsubsection{CONCLUSION}

In conclusion, ECGI-based localization of PVCs has the potential to support ablation therapy, improving localization accuracy, reducing procedural times and reducing the number of ablation applications. Due to its per-beat result, also infrequent PVCs can be successfully mapped with ECGI. Ultimately, ECGI could help to improve success rate of ablation therapy.

\subsection{IMAGE INTEGRATION}

Arrhythmia mechanisms require a substrate and a trigger. Figure 7.11 shows a case in which the trigger was presumed to be in myocardial scar, as MRI showed regions of late enhancement. The trigger was localized with ECGI. After fusion of ECGI and MRI, it was apparent that the ECGI-based PVC origin was not localized within the region of late enhancement. The patient subsequently underwent an EP study. Invasive voltage mapping with Carto showed no significant regions of abnormal potential amplitudes, suggesting that the late enhancement from MRI had limited electrical consequences. Optimal ablation locations were determined by activation mapping and pace mapping. These locations were outside the region of late enhancement from MRI, further confirming the limited role of late enhancement in this case. The ablation locations with highest suspicion for PVC origin (earliest EAT, highest pace mapping score) were localized within $19 \mathrm{~mm}$ from the predicted PVC origin. Again, this distance is partially explained by the thickness myocardial wall due to the endocardial location of ablation, and epicardial location 


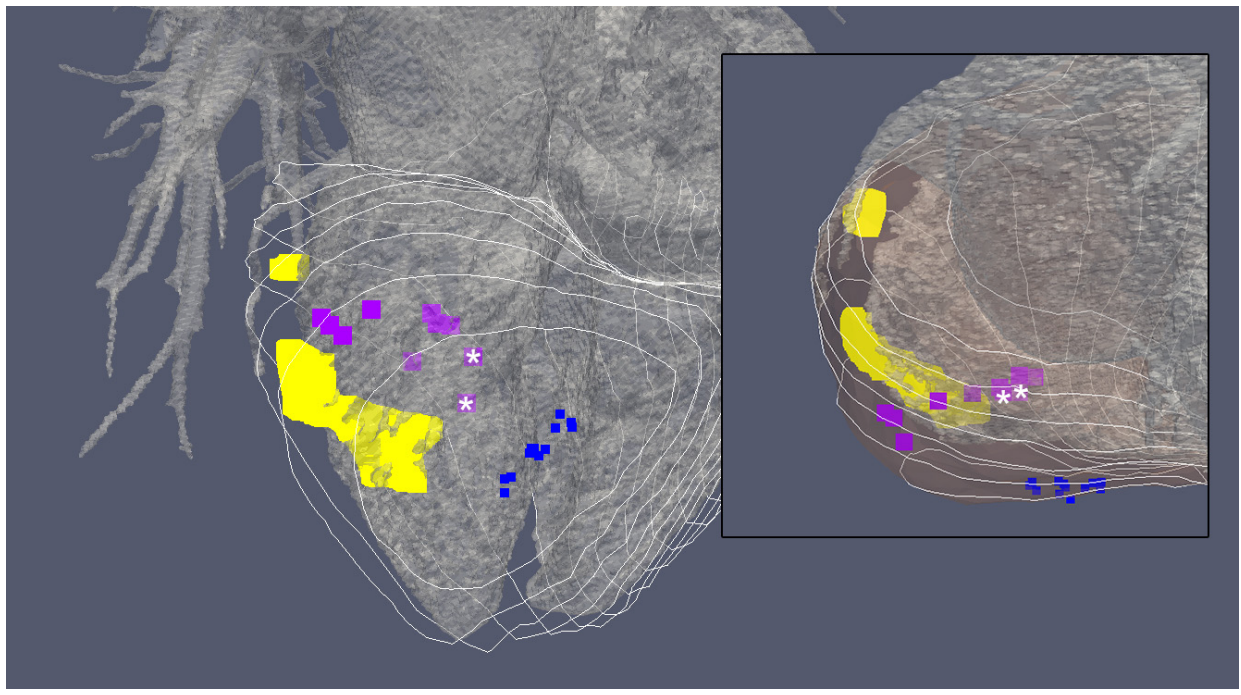

Figure 7.11: Image integration of CT, ECGI, MRI and Carto in a PVC ablation case. The contrast pool from CT shows the anatomy of the intracardiac and intravascular structures (gray). The isochrone lines show the ECGI-based activation sequence of a PVC on the epicardium (white). The ECGI-reconstructed origins of multiple PVCs are projected on the epicardium as blue squares. The region of late enhancement as segmented from MRI is depicted in yellow. The endocardial ablation locations (registered with the Carto threedimensional mapping system) are shown as purple squares; those with the highest pace map score $(12 / 12,97 \%)$ and earliest EAT are marked with an asterisk. Inset shows a different view.

of PVC prediction (see inset in Figure 7.11). Additionally, the fusion of four imaging modalities (CT, MRI, ECGI, and Carto) will have a lower spatial accuracy than the individual modalities.

This shows that image integration will likely become an important part of clinical therapy. As is evident from the previous case, a substrate that is visible with imaging modalities (e.g., the suggestion of myocardial scar from MRI) need not always correspond to results from electrical imaging with ECGI. For example, late enhancement on MRI is not always the result of myocardial scar tissue. Combined use of tissue imaging with MRI and electrical imaging with ECGI can improve ablation success, both when the different modalities show correspondence between electrical and tissue abnormalities, and when there is no correspondence.

Other research groups have also highlighted the use of image integration in arrhythmia therapy. Cochet et al [181] combined MRI with ECGI for noninvasive assessment of cardiac arrhythmias. Their output was a threedimensional cardiac model integrating cardiac anatomy, myocardial substrate, and epicardial activation. They were able, as we were, to integrate this in navigation systems and guide mapping and ablation. [181] 
More recently, they used a similar approach, but without ECGI, to visualize the left phrenic nerve and coronary arteries during ventricular tachycardia ablation. [182] These structures should not be damaged during ablation and high-resolution image integration for procedural visualization can therefore prevent complications. In another study, they also integrated wall thinning from CT, late enhancement from MRI, and myocardial hypoattenuation from CT. [183] They showed that the integrated information prompted to adapt or intensify the mapping procedure in $57 \%$ of the cases, resulted in an epicardial approach in $33 \%$, and modified epicardial ablation strategy (due to proximity of coronary arteries or phrenic nerve) in $43 \%$ of the patients. One can imagine that additional integration of noninvasive electrical imaging with ECGI further influences mapping and ablation strategies and might improve outcome.

\subsection{THE ROLE OF RECOVERY ABNORMALITIES IN ARRHYTHMIA MECHANISMS}

Sudden cardiac death has been identified as one of the current challenges of the field of cardiac arrhythmias. [184] One fifth of deaths is sudden, and a majority is likely to be of cardiac origin. SCD is particularly important in young individuals, explaining two-thirds of the sudden deaths in young individuals ( $\leq 40$ years). [185]

As discussed in this thesis, and especially in Chapter 6, abnormalities in electrical recovery of the heart can form an important substrate for cardiac arrhythmias and SCD. [131], [133]-[137] Recovery is usually gradual and smooth, as demonstrated for humans in the literature [73], [154], and for both normal and abnormal beats in canines in this thesis (Section 3.8). Neighboring areas with different repolarization times provide a substrate for re-entry tachycardia and reduce the threshold for VF. [131] However, such recovery gradients are difficult to assess on the 12-lead ECG: a proarrhythmic steep gradient of repolarization may occur in a small area that is not reflect by QT dispersion on the 12-lead ECG. Conversely, repolarization differences that are far apart on the heart surface might be visible on the 12-lead ECG as QT dispersion, but will likely not create a proarrhythmic local steep repolarization gradient. [131] As shown in this thesis, ECGI overcomes the low spatial resolution of the 12-lead ECG. Due to the increased spatial resolution and anatomical information provided by ECGI at the organ level, it could be better suited to detect repolarization abnormalities. In Chapter 6, we have shown that ECGI can image and localize steep recovery gradients as electrical substrate of an arrhythmia that was not apparent from the 12-lead ECG.

ECGI has been used previously to investigate recovery abnormalities on the organ level. In long QT syndrome [73] and Brugada syndrome [154], it was shown that patients had abnormally steep gradients of repolarization. Repolarization gradients were 
steeper in symptomatic than in asymptomatic patients. [73] Additionally, ECGI was used to characterize early recovery. [72] In that case report, two patients with signs of early recovery on the 12-lead ECG were shown to have steep local repolarization gradients with local early repolarization.

We expect that investigation of the role of recovery abnormalities will bring more insight in mechanisms of arrhythmogenesis and could improve risk prediction for SCD.

\subsection{Conclusions ANd FUtUre WORK (PARt II)}

We have employed ECGI in PVC ablation therapy in selected patients. ECGI can guide invasive PVC and VT ablation, especially when arrhythmias are infrequent during the procedure. In the literature, ECGI has been shown to reduce procedural time and ablation applications. Future studies are needed to investigate whether ablation success rate is improved as well. Image integration will play a key role in advanced arrhythmia imaging for ablation therapy.

Additionally, we have shown that ECGI can be used to image the arrhythmia substrate in patients with (suspected) recovery abnormalities. Preliminary applications at MUMC+ have successfully guided therapy. We aim to extend this approach to larger populations of patients with (suspected) recovery abnormalities, e.g., carriers of gene mutations in cardiac ion channels. ECGI will shed light on the normal and abnormal recovery of the heart. We expect that detection of local, steep gradients of repolarization will be associated with risk for cardiac arrhythmias, and that PVCs that follow closely after abnormal recovery can be arrhythmogenic if the steep recovery gradient functions as line of conduction block.

Further insights might be gained when combining ECGI with other imaging modalities, such as CT and MRI. Functional CT scanning will facilitate the incorporation of cardiac mechanics in ECGI. ECGI is currently based on a static heart geometry, and we have shown that this simplification has a negative correlation with the quality of reconstructed epicardial potentials. Including cardiac mechanics will likely improve accuracy further, and is essential to investigate electro-mechanical and mechano-electrical coupling in arrhythmogenesis. Tissue characterization with MRI will shed light on the relation between tissue characteristics (e.g., myocardial scar) and electrical (recovery) abnormalities.

Multi-scale integration can be achieved by investigating relevant disease mechanisms on a cellular scale. Regional APD differences can result from heterogeneous expression of different ion channels, which can be exacerbated by genetic or acquired factors, thereby promoting arrhythmogenic repolarization abnormalities. Computational modeling has 
proven useful to analyze the potential proarrhythmic effects of ion-channel dysfunction under a variety of conditions.

In short, we propose to investigate the following hypotheses:

1. ECGI-guided PVC ablation, ideally supported by integration of multiple imaging modalities, can improve therapy outcome.

2. Local steep repolarization gradients can form the vulnerable substrate for arrhythmias in some patients; this may not be visible on the 12-lead ECG but can be imaged with ECGI.

3. Short-coupled PVCs in co-existence with a recovery substrate may result in $\mathrm{VT} / \mathrm{VF}$ in severe cases, due to functional conduction block in the region of steep recovery gradient followed by reentry.

4. Translational research combining ECGI with in vitro and in silico cellular studies brings new insight in the role of ion channelopathies in arrhythmia substrate and trigger mechanisms.

5. Incorporation of cardiac mechanics in ECGI improves imaging of recovery abnormalities.

Ultimately, our long-term aim is to arrive at insights and methods that provide better risk stratification and improved treatment of patients potentially susceptible to cardiac arrhythmias and sudden cardiac death.

\section{ACKNOWLedgments}

The author would like to thank Laurent Pison, $\mathrm{MD}, \mathrm{PhD}$, and Harry Crijns, $\mathrm{MD}, \mathrm{PhD}$, for referring patients for the ECGI/ablation study. 

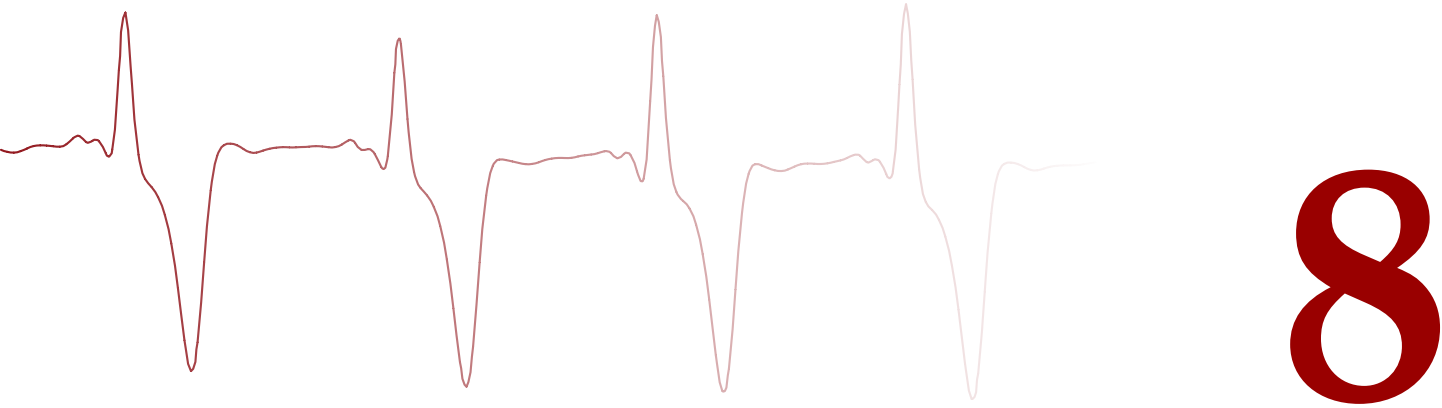

Summary 
The rhythmic contraction of the heart is governed by electrical impulses. Perturbations of the normal rhythm are called cardiac arrhythmias. If cardiac arrhythmias cause inadequate pumping of the heart, they can be life-threatening and require restoration of normal rhythm.

The electrical activity of the heart propagates as an electromagnetic field to the body surface, where it can be recorded as an electrocardiogram (ECG). The ECG is a wellestablished, patient-friendly, quick, reproducible, and cheap tool to determine normal cardiac activation and recovery, and diagnose cardiac arrhythmias and other conditions. However, it lacks the capacity to directly assess electrical activity at the level of the heart muscle at high spatial resolution.

Conversely, noninvasive electrocardiographic imaging (ECGI) is a technique that employs mathematical formulations to reconstruct the electrical activity directly at the level of the heart muscle, from extensive body-surface electrocardiograms and a digitized, patient-specific body and heart geometry. It uses a model of the propagation of the electromagnetic field (from the heart to the body surface) to reconstruct the electrical source of the recorded body-surface ECGs. The development, validation and application of this technique represent the main topics of this thesis. Application of ECGI consists of the following steps, as illustrated in Figure 8.1:

1. Recording of multiple body-surface ECGs for high-density mapping.

2. Imaging the patient's heart and torso to obtain the position of the electodes relative to the heart.

3. Digitizing the patient's torso-heart geometry, and mapping the recorded bodysurface potentials on the torso.

4. Noninvasive reconstruction of the potentials on the heart surface from the recorded body-surface potentials. This process is hindered by mathematical instability and requires additional constraints to obtain stable and accurate solutions.

5. Computing electrical activation and recovery of the heart (represented by isochrones) from noninvasively reconstructed potentials at the heart surface.

Chapter 2 reviews all of the above steps and discusses the methodologies that are commonly applied.

The Maastricht implementation of ECGI is described and validated in Chapter 3. In an animal study, invasive recordings on the heart surface were obtained, and compared to noninvasive reconstructions with ECGI. This study shows that ECGI reconstructs electrograms at the heart surface with a high median accuracy, but with considerable variability. This variation can be explained by a spatial mismatch, which is overall $<20 \mathrm{~mm}$. Variability in electrogram quality is adequately addressed by a spatiotemporal approach 

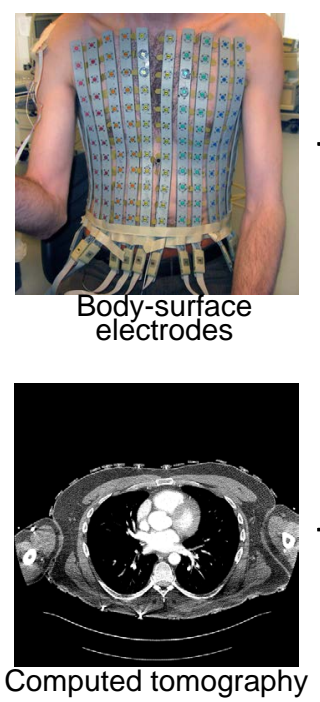
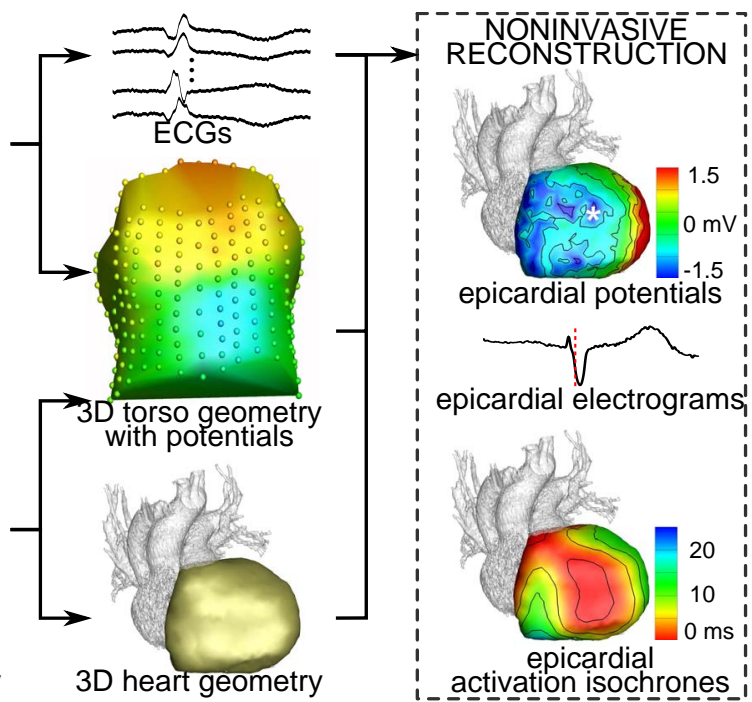

Figure 8.1: Electrocardiographic Imaging (ECGI) noninvasively reconstructs electrograms and activation and recovery isochrones on the heart surface. Body-surface ECGs are combined with a torso-heart geometry obtained with CT. By carefully reversing the physical laws of electromagnetism, epicardial potentials can be reconstructed. From these, epicardial electrograms and the sequence (depicted by isochrones) of activation and recovery are subsequently computed.

that incorporates the characteristics of neighboring electrograms as well, enabling more accurate derivation of activation and recovery times. The origin of artificially-induced beats can be determined with a median error of $10 \mathrm{~mm}$, which, upon translation to a clinical setting, could potentially expedite catheter-based diagnostic evaluation and ablation of abnormal beats, as discussed later.

One of the methods introduced in Chapter 3 deals with computation of the activation and recovery times from the electrogram reconstructed at each virtual point on the heart geometry. We show that a method taking into account the electrograms of neighboring nodes results in more accurate computation of activation and recovery times. The specifics of this method are investigated in more detail in the Chapter 7 .

In Chapters 4 and 5 novel cardiac source models are investigated. Cardiac source models are the representations of the electrical activity at the heart, for example, as local potentials at the heart surface. Our novel methods do not directly reconstruct potentials at the heart surface, but employ different models of the cardiac electrical activity that allow for a more efficient (sparser) representation. A sparse representation of electrical activity at the heart makes ECGI less sensitive to noise. 
The physiology-based method (Chapter 4) is inspired by 'physiologically-realistic' spatial patterns of potentials on the heart surface. These patterns are simulated by computational models of the electrical activity of cardiac muscle cells. The subsequent potential patterns are then used as building blocks for the reconstruction of actual potentials on the heart surface. This novel method was compared to traditional methods and validated using the invasive data of Chapter 3. It recovers details of heart-surface electrograms that are lost with traditional methods, attains higher correlation coefficients and leads to improved estimation of recovery times. The best results are obtained by including approximate knowledge about the beat origin and selecting only the appropriate building blocks.

Chapter 5 describes the 'wavelet-based method'. Wavelets are oscillations with certain mathematical properties, and can be used as building blocks for time signals. These temporal building blocks describe the reconstructed potentials more efficiently. The method pursues a sparse wavelet representation over the time course of an electrogram, and simultaneously exploits the spatial relationship between local electrograms. When compared to traditional methods, this novel method yields potentials at the heart surface with higher accuracy, and more accurate reconstruction of recovery times.

Thus, the most important improvement by these sparsity-based methods is an increased accuracy of reconstructed recovery times, which is relevant when investigating the influence of recovery abnormalities on the development of cardiac arrhythmias in patients.

After these technical and mathematical innovations, we subsequently applied ECGI for two clinical purposes: 1) investigation of patient-specific mechanisms of cardiac arrhythmias; and 2) guidance of ablation therapy in a clinical setting.

In Chapter 6, ECGI is applied to a clinical case to obtain new, patient-specific insights in the mechanisms that can lead to arrhythmias. In this specific patient, the presence of abnormalities in electrical recovery of the heart appears to play a role in the development of life-threatening arrhythmias. These recovery abnormalities could not be detected from the clinical ECG, but were identified using the noninvasive ECGI reconstruction. The presence of a large dispersion of recovery times (or, in other words, large variability in recovery times in a small area of tissue) can lead to functional reentry. Functional reentry is a known mechanism of arrhythmogenesis and can lead to life-threatening arrhythmias such as ventricular tachycardia and ventricular fibrillation. An abnormal electrical recovery substrate may be dangerous when premature beats are present, as in this case, because this can promote functional reentry. This chapter also demonstrates the added value of a translational approach, by combining ECGI with computational modeling of the effects of genetic mutations of ion channels in cardiac muscle 
cells.

Premature ventricular contractions (PVCs) are beats that do not arise from the sinus node, but from tissue that normally does not generate beats independently. These beats activate the heart slowly and asynchronously. A high burden of PVCs can necessitate treatment. Invasive ablation therapy can eliminate the tissue that generates these beats. Ablation therapy is performed by introducing a catheter in the patient's heart, localizing the culprit tissue, and subsequent silencing that tissue by applying radiofrequency energy, or high or cold temperatures. The general success rate of PVC ablation is suboptimal, partially due to the difficulty of localizing the culprit tissue, especially when no or limited PVCs are present during the ablation procedure. In Chapter 7, we describe the application of ECGI to pre-procedurally predict the culprit lesion. The noninvasively reconstructed activation map of the PVC is then used during the ablation procedure to guide localization and ablation of these abnormal impulses.

Additionally, Chapter 7 puts the results obtained in this thesis in perspective. We highlight the importance of technical developments to further ECGI and improve accuracy. Amongst others, we propose to include cardiac mechanics in ECGI in future studies.

To obtain more insights in cardiac arrhythmogenesis, we propose to further investigate the relationship between recovery abnormalities and cardiac arrhythmias. Translational studies that combine computational or wet-lab studies of cellular electrophysiology, advanced image integration at tissue and organ level, and ECGI will provide considerably more knowledge than studies that focus on single aspects alone. 

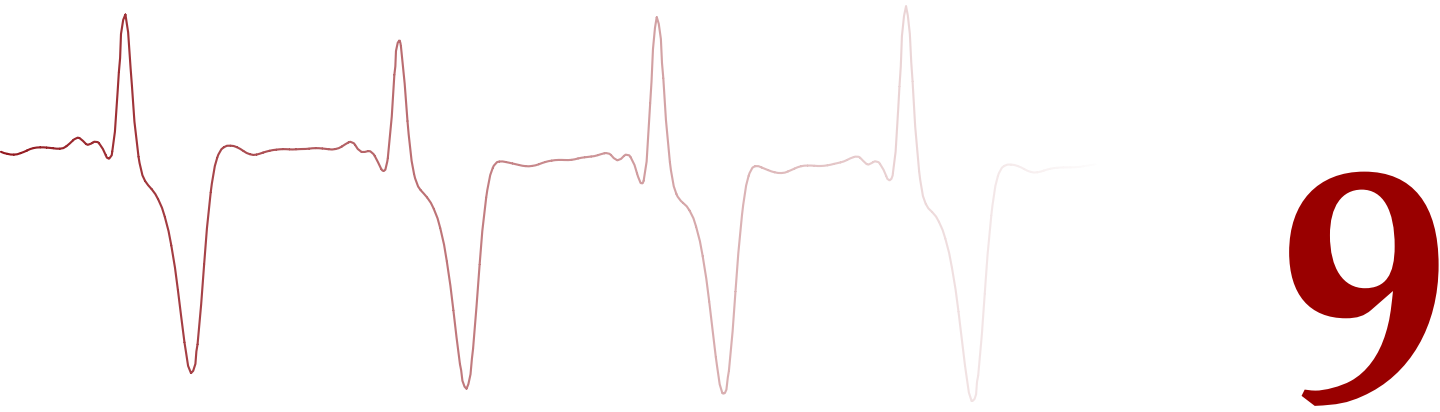

Samenvatting 
De ritmische samentrekking van het hart wordt door elektrische impulsen aangestuurd. Verstoringen van dit ritme worden hartritmestoornissen genoemd. Wanneer hartritmestoornissen de pompfunctie van het hart ernstig verstoren, kan dit levensbedreigend zijn. In dat geval moet het normale ritme hersteld worden.

De elektrische activiteit van het hart propageert als een elektromagnetisch veld naar het lichaamsoppervlak, waar het kan worden opgenomen als een electrocardiogram (ECG), ook wel bekend als het 'hartfilmpje'. Het ECG is een gevestigde, patiëntvriendelijke, snelle, reproduceerbare en goedkope techniek om de normale en abnormale activiteit van het hart te onderzoeken. Het ECG is echter niet in staat de elektrische activiteit in hoge resolutie op het niveau van het hart zelf zichtbaar te maken.

Dit is wel mogelijk met de eveneens niet-invasieve techniek 'electrocardiographic imaging' (ECGI), ofwel elektrocardiografische beeldvorming. ECGI is een techniek die door middel van wiskundige beschrijvingen in staat is de elektrische activiteit van het hart direct op de hartspier te reconstrueren, gebaseerd op uitgebreide metingen van ECGs op het lichaamsoppervlak en een digitale hart-torso geometrie van de patiënt. ECGI gebruikt een fysisch model van de propagatie van het elektromagnetische veld (van het hart naar de torso) om de elektrische bron op het hart te reconstrueren die leidt tot de gemeten ECGs. De ontwikkeling, validatie en toepassing van deze techniek vormen de belangrijkste onderwerpen van dit proefschrift. Het uitvoeren van ECGI omvat de volgende stappen, zoals ook zichtbaar in Figuur 9.1:

1. Het opnemen van een groot aantal ECGs op het lichaamsoppervlak.

2. Scannen van het hart en de torso van de patiënt, om de positie van de elektroden ten opzichte van het hart te bepalen.

3. Het digitaliseren van de patiënt-specifieke hart-torso geometrie, en het koppelen van de ECGs aan de gedigitaliseerde torso.

4. Niet-invasieve reconstructie van de potentialen op het hartoppervlak, bepaald uit de gemeten potentialen op het lichaamsoppervlak. Dit proces wordt bemoeilijkt door wiskundige instabiliteit, en vereist extra selectie binnen alle mogelijke oplossingen, om zo tot een stabiel en accuraat resultaat te komen.

5. Het berekenen van de volgorde van activatie (depolarisatie) en herstel (repolarisatie) op het hartoppervlak (weergegeven door isochronen) uit de gereconstrueerde potentialen van het hart.

Hoofdstuk 2 bespreekt deze stappen en de meest gebruikte methoden.

In Hoofdstuk 3 wordt beschreven hoe ECGI in Maastricht is opgebouwd en gevalideerd. Invasieve metingen op het hartoppervlak werden verricht in een dierstudie, en vergeleken met niet-invasieve reconstructies van ECGI. Deze studie toont aan dat ECGI 

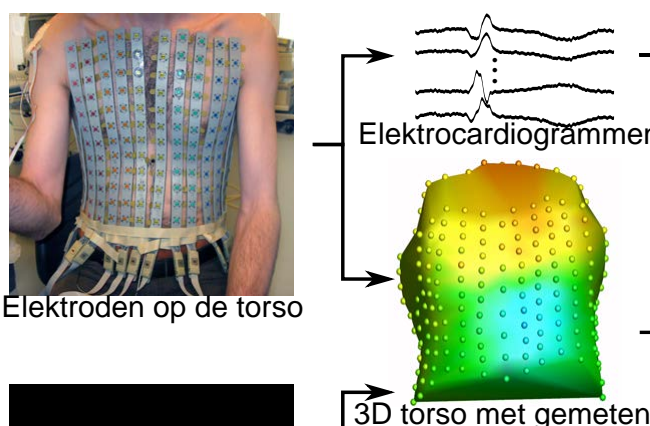

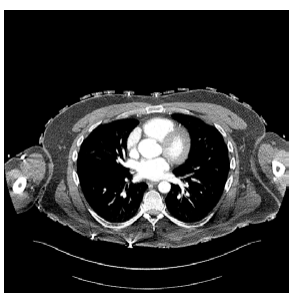

CT scan
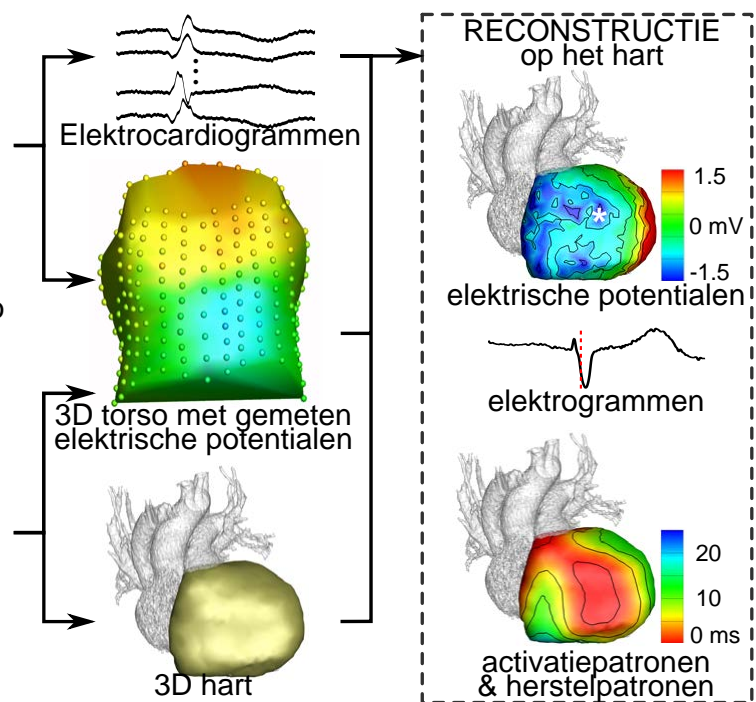

Figuur 9.1: Electrocardiographic Imaging (ECGI) reconstrueert electrogrammen en patronen van elektrische activatie en herstel direct op het hartoppervlak. ECGs gemeten op het lichaamsoppervlak worden gecombineerd met een torso-hart geometrie uit een CT scan. Door vervolgens gecontroleerd de fysische wetten van elektromagnetisme om te keren, kan men potentialen op het hartoppervlak reconstrueren. Hieruit kan vervolgens de volgorde van elektrische activatie (depolarisatie) en herstel (repolarisatie) van het hart berekend worden, en middels isochronen afgebeeld worden.

electrogrammen reconstrueert op het hartoppervlak met een hoge gemiddelde nauwkeurigheid, maar met een aanzienlijke variabiliteit. Deze variabiliteit kan verklaard worden door een verschuiving van de gereconstrueerde elektrogrammen ten opzichte van de gemeten elektrogrammen; deze verplaatsing is in het algemeen kleiner dan $20 \mathrm{~mm}$. Wanneer men de elektrische depolarisatie- en repolarisatietijden uit deze elektrogrammen bepaalt, kan de variabiliteit in electrogramkwaliteit goed aangepakt worden door informatie over naburige elektrogrammen mee te nemen. Dit leidt tot een accurate bepaling van de activatie- en herstelpatronen. Verder blijkt dat de origine van een hartslag met een mediane fout van $10 \mathrm{~mm}$ bepaald kan worden. Wanneer men dit vertaalt naar de klinische praktijk, zou dit kunnen betekenen dat katheter-gebaseerde diagnostiek en therapie hiermee gestuurd zou kunnen worden, zoals we later zullen beschrijven.

In Hoofdstukken 4 en 5 worden nieuwe 'bronmodellen' voor de elektrische activiteit van het hart onderzocht. Bronmodellen zijn een representatie van de elektrische bron in het hart, die op het lichaamsoppervlak tot de ECG metingen leidt. In het tot nog toe beschreven bronmodel vormen de potentialen op het hartoppervlak de bron van de potentialen op het lichaamsoppervlak. De nieuwe bronmodellen reconstrueren niet di- 
rect hartpotentialen, maar gebruiken andere voorstellingen die tot een meer efficiënte ('spaarzame') representatie leiden. Een efficiënte representatie van elektrische hartactiviteit zorgt ervoor dat de ECGI reconstructie minder gevoelig is voor de invloed van ruis.

De 'physiology-based regularization' methode (Hoofdstuk 4) is gebaseerd op realistische patronen van potentiaalverdelingen op het hart. Deze potentiaalverdelingen zijn gesimuleerd met computermodellen van de elektrische activiteit van hartspiercellen. De meest voorkomende potentiaalverdelingen uit de simulaties werden vervolgens gebruikt als bouwstenen voor de reconstructie van de daadwerkelijke potentialen. Deze nieuwe methode werd vergeleken met traditionele methodes en gevalideerd in de invasieve data van Hoofdstuk 3. De methode blijkt in staat om meer detail te behouden in de gereconstrueerde electrogrammen dan traditionele methoden, en met name tijden van elektrische repolarisatie beter te bepalen. De beste resultaten worden behaald wanneer men informatie heeft over de mogelijke origine van de hartslag, en de bouwstenen daarop selecteert.

Hoofdstuk 5 beschrijft de 'wavelet-based method'. Wavelets zijn oscillaties met bepaalde wiskundige kenmerken, en kunnen gebruikt worden als bouwstenen voor tijdsignalen. De nieuwe methode beschrijft de temporele karakteristieken van electrogrammen door middel van wavelets, en maakt tegelijkertijd gebruik van de ruimtelijke relatie tussen de potentialen. In deze gecombineerde spatiële-temporele methode wordt een efficiënte representatie in zowel ruimte als tijd nagestreefd. In vergelijking met traditionele methoden levert deze nieuwe methode potentialen met hogere nauwkeurigheid, die vooral tot een betere berekening van repolarisatietijden leidt.

Zo is de belangrijkste verbetering die bereikt wordt met deze twee nieuwe bronmodellen een nauwkeurigere bepaling van de repolarisatietijden. Dit is relevant voor onderzoek naar de invloed van repolarisatieafwijkingen op de ontwikkeling van hartritmestoornissen van patiënten.

$\mathrm{Na}$ deze technische en wiskundige innovaties hebben we vervolgens ECGI toegepast voor twee klinische doeleinden: 1) onderzoek naar de patiënt-specifieke mechanismen van hartritmestoornissen; en 2) het sturen van ablatietherapie in de kliniek.

In Hoofdstuk 6 wordt ECGI toegepast in een klinische casus om nieuwe, patiëntspecifieke inzichten te verkrijgen in de mechanismen die leiden tot hartritmestoornissen. In deze specifieke patiënt lijkt de aanwezigheid van afwijkingen in het elektrisch herstel een rol te spelen bij de ontwikkeling van een levensbedreigende ritmestoornis. Deze repolarisatieafwijkingen waren niet zichtbaar op het klinische ECG, maar konden worden geïdentificeerd met ECGI. De aanwezigheid van grote variabiliteit in repolarisatietijden in een klein gebied van weefsel kan leiden tot functionele re-entry. Een re-entry ritmestoornis is een situatie waarbij de elektrische activatie van het hart niet uitdooft, maar 
in cirkelachtige patronen kan blijven doorgaan. Dit kan leiden tot levensbedreigende ritmestoornissen, bijvoorbeeld ventriculaire tachycardie en ventrikelfibrillatie. De aanwezigheid van repolarisatieafwijkingen is mogelijk van extra belang wanneer er premature hartslagen zijn, zoals bij deze patiënt ook het geval was, aangezien dit waarschijnlijk sneller tot functionele re-entry leidt. Dit hoofdstuk toont ook de toegevoegde waarde van een translationele benadering, door het combineren van ECGI met computationele modellen van de effecten van genetische mutaties van ionkanalen in hartspiercellen.

Premature ventriculaire contracties (PVC's) zijn hartslagen die niet uit het normale geleidingsysteem van het hart komen, maar ontstaan in weefsel dat normaal niet tot spontane hartslagen leidt. Doordat het normale geleidingsysteem niet betrokken is, is de activatie van het hart langzaam en asynchroon. Wanneer men veel PVC's vertoont, kan een behandeling noodzakelijk zijn. Invasieve ablatietherapie kan het weefsel dat PVC's genereert elektrisch uitschakelen. Ablatie wordt uitgevoerd door het inbrengen van een katheter in het hart van de patiënt, waarna het PVC-genererend weefsel wordt gelokaliseerd en vervolgens wordt uitgeschakeld door toepassing van radiofrequente energie of zeer hoge of lage temperaturen. Het succespercentage van PVC ablatie is niet optimaal, mede doordat het moeilijk kan zijn om het slechte weefsel te lokaliseren, vooral wanneer er geen of weinig PVC's tijdens de procedure aanwezig zijn. In Hoofdstuk 7, beschrijven we de toepassing van ECGI om vóór de ablatieprocedure al de locatie van de PVC te bepalen, om zo de ablatie te kunnen sturen.

Daarnaast plaatst Hoofdstuk 7 de resultaten die in dit proefschrift werden behaald in perspectief. We benadrukken het belang van verdere technische ontwikkelingen om tot nauwkeurigere resultaten te komen, zoals bijvoorbeeld het meenemen van de beweging van het hart in de ECGI procedure.

Om meer inzicht te krijgen in hartritmestoornissen, stellen we daarnaast voor om de relatie tussen repolarisatieafwijkingen en ritmestoornissen verder te onderzoeken. Het is hierbij van belang om deze studies translationeel uit te voeren, middels computationele modellen of levende cellen om de cellulaire elektrofysiologie te onderzoeken, en te combineren met geavanceerde integratie met beeldvorming op weefselniveau, en met ECGI. Deze integratieve combinatie van technieken zal ons meer kennis brengen dan de individuele technieken op zich. 
Cardiovascular disease remains a major cause of death and disability in western society. The results obtained in this thesis could bring improvements in screening, diagnosing and treatment of (the risk for) cardiac arrhythmias. Apart from a social burden, cardiac arrhythmias also create an economic burden on society. This chapter describes the valorization possibilities based on the knowledge obtained in this thesis. Knowledge valorization is "translating academic wisdom to societal benefit," and can be achieved by a broad range of activities and products. Examples includes patents, licenses, software, making models and systems available, co-publications with social or economic stakeholders, publications in journals and newspapers or other non-academic media, advice for organizations or companies, training programs, spin-offs and start-up companies. The results obtained in this thesis have a clear, direct clinical benefit, but could also be valorized in several indirect ways.

\subsection{SCREENING FOR SUDDEN CARDIAC DEATH}

In the United States of America (USA) alone, approximately 340,000 individuals per year die from sudden cardiac arrest. [186] Sudden cardiac death (SCD) is particularly important in young individuals, explaining two-thirds of the sudden deaths in individuals $\leq 40$ years [185]. Although it seems likely that (primary) cardiac arrhythmias are a major player in SCD in the general population, and especially in young individuals, it remains a challenge to assess the risk for SCD in apparently healthy individuals.

Electrocardiographic imaging (ECGI), the technique discussed in this thesis, might help to improve risk assessment for SCD in apparently healthy individuals, as it provides a detailed image of the electrical activity and recovery at the level of the heart surface. As discussed in Chapters 6 and 7, ECGI might detect arrhythmogenic substrate that is not apparent from screening tools such as the 12-lead electrocardiogram (ECG). More studies are needed to investigate whether ECGI indeed has a higher sensitivity and specificity to detect risk factors for SCD than the 12-lead ECG. Initial studies should focus on homogeneous groups of patients, e.g., with genetic mutations in cardiac ion channels for which the risk of SCD is not yet known. When the added benefit of ECGI is shown in these selective studies, risk factors for SCD could be studied in the general population.

ECGI's hypothesized improved sensitivity for SCD would help to treat and protect individuals in which risk factors for SCD are not detected with normal screening tools. This could prevent unnecessary death and disability, and the associated socioeconomic costs. Improved specificity would help to reduce unnecessary treatment of healthy individuals, for example by reducing the number of implantations of implantable 
cardioverter defibrillators (ICDs), thereby decreasing their psychological and economic burden.

Thus, the target groups for this application consist of patients and their family members, who receive more information about their risk for SCD; and cardiologists, who receive better tools to determine that risk and adjust treatment for their patients.

The socioeconomic effect of ECGI should be studied to determine its feasibility as a screening tool. The application of ECGI is time consuming and expensive, and studies are needed to investigate whether the cost (extensive screening) are outweighed by the benefits (reduction in SCD and unnecessary treatment).

\subsection{OPPORTUNITIES FOR PERSONALIZED AND TRANSLATIONAL MEDICINE}

ECGI allows to study mechanisms of arrhythmia, and the effect of treatment, in a personalized matter. An example of this is shown in Chapter 6, where the application of ECGI helped hypothesize possible mechanisms of induction of arrhythmia in a patient. Such insights can help to provide a better, more specific diagnosis for a patient's symptoms. Additionally, these data help to select the best treatment. Moreover, the effect of treatment could be assessed with ECGI.

Additionally, ECGI can help to bridge the gap between studies on cellular level and effects on organ level. For example, studies investigating the effect of genetic mutations on cellular level could provide hypotheses on organ level that can be investigated with noninvasive reconstructions of potentials at the heart surface with ECGI. This would allow to study the phenotypic results of genetic mutations in the intact organ in humans.

The target audience for this application again consists of patients and cardiologists, who are informed better of diagnosis and treatment options. Additionally, scientists can benefit from new opportunities to translate cellular findings to organ studies.

\subsection{IMPROVED ABLATION GUIDANCE}

As discussed extensively in Chapter 7, premature ventricular contractions (PVCs) are arrhythmias that might require treatment, as they can impair the pumping function of the heart. Additionally, sick myocardial tissue can generate ventricular tachycardia (VT) that can be life threatening. However, the non-medicinal treatment of PVCs and VTs with invasive ablation therapy is still suboptimal. One of the reasons is the difficulty in localizing the culprit tissue during the ablation procedure, especially when the patient shows few or no PVCs or VTs during the procedure. ECGI can determine the origin of a PVC in a single beat, and can be performed pre-procedurally. This pre-procedural pre- 
diction can be visualized during the ablation procedure and helps to guide the ablation, as discussed in Chapter 7. Previous studies have shown that this reduces the time needed for the procedure. However, the success rate of ECGI-guided PVC ablation appears to be similar to non-guided ablation in preliminary studies. Thus, more studies are needed to investigate whether there is a socioeconomic benefit of using ECGI during PVC ablation.

Ultimately, improved treatment of PVC and VT would increase quality of life and might reduce the need for ICD implantation or repetition of unsuccessful ablation procedures.

A recent, preliminary study investigated completely noninvasively cardiac ablation by using radiotherapy. [176] For this application, catheter-based confirmation of the culprit tissue is unavailable, and ECGI provides a noninvasive alternative. The initial results should be confirmed in larger cohorts, and could initiate a new era to arrhythmia treatment by allowing for completely noninvasive diagnosis and treatment of cardiac arrhythmias. Clearly, this would allow for commercial exploitation of modalities that combine ECGI with radiotherapy.

\subsection{APPLICATION OUTSIDE THE CARDIAC DOMAIN}

Inverse problems are common outside the cardiac domain. For example, recordings of electrical signals on the scalp can be used to investigate the electrical activity of the brain. Other organs that exhibit electrical activity that could be noninvasively reconstructed include the stomach, intestines, uterus and skeletal muscles. The novel source models described in Chapters 4 and 5 could be applied to these inverse problems as well, especially when these problems can be expressed in a sparse way.

The spatiotemporal method introduced in Chapter 3 , used to determine the moment of electrical events from reconstructed potentials, might be beneficial in those cases as well, especially when there is a clear natural relationship between neighboring signals on the organ's surface, for example, because they resemble an activation wave front.

\subsection{COMMERCIAL BenEFits}

The Maastricht implementation of ECGI is well investigated and validated, with a special (and novel) focus on recovery abnormalities. With the opportunities expressed in the previous paragraphs, commercial exploitation seems a logical prospect. An ideal ECGI system for clinical application is easy-to-use, allows a flexible attachment of electrodes on a variety of torso shapes, enables quick creation of a torso-heart geometry from CT scans, and provides a live visualization of inverse reconstruction during ablation procedures, electrophysiology studies, drug challenges and for screening purposes. 

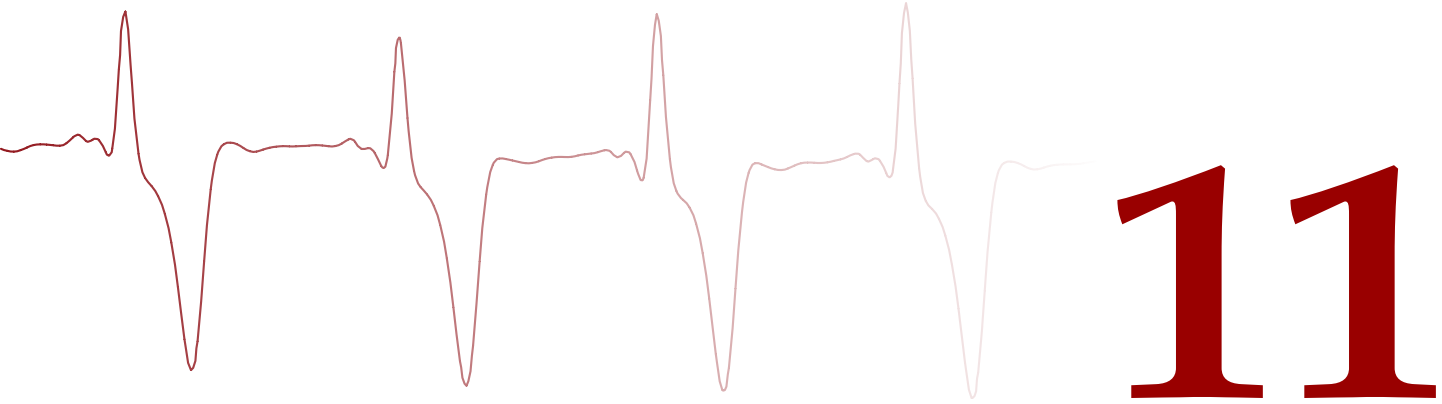

Acknowledgements / Dankwoord 
Allereerst, en niet alleen omdat het hoort, wil ik mijn promotoren bedanken. Ik ben een van de weinige gelukkigen met drie promotoren (en geen copromotoren). Soms betekent dat driemaal zo lang wachten tot een knoop is doorgehakt, maar meestal betekent het meer dan driemaal zo veel interessante, stimulerende en productieve interactie (en ook de mogelijkheid om de feedback van één promotor te negeren ten faveure van de andere promotoren, of vaker, ten faveure van mijn eigen mening). Het begrip "dagelijkse supervisoren" zal veel promovendi enigszins ironisch in de oren klinken; weinigen zijn gezegend (of belast?) met dagelijkse bemoeienis van hun begeleiders. Mijn promotoren Ralf Peeters, Paul Volders, en Ronald Westra heb ik zelden wekelijks, laat staan dagelijks gezien, maar hadden ieder op hun eigen manier toch vrijwel dagelijks invloed op mijn analyses en ideeën.

Ralf, niet alleen heeft jouw wiskundig inzicht een belangrijke inhoudelijke bijdrage geleverd aan deze scriptie, je rustige houding en je vermogen om de materie te overzien en te relativeren hebben mij veel gebracht. Daarnaast zal ik niet vergeten hoe jij mij begeleidde op een internationaal congres. En al was dit welliswaar slechts een uur rijden in België, jouw rustgevende aanwezigheid maakte deze eerste ervaring een stuk prettiger.

Paul, niet zelden heb je enige overreding moeten gebruiken om mij de gewenste richting op te krijgen, maar vaak bleek dit juist een schot in de roos. Je inhoudelijke overwegingen, in hun breedvoerige formulering, bleken vaak zeer relevant. Je vlijmscherpe schrijfstijl heeft ons werk naar een hoger niveau getild, al was de benodigde feedbacktijd daardoor vaak een uitdaging voor mijn geduld. De waarde die je hecht aan sociale binding binnen een team hebben geresulteerd in vele gezellige en culinair hoogstaande etentjes, en een team dat ondanks hun inhoudelijk vaak grote verschillen een sterke onderlinge binding kent. Vasthoudend heb je me gedwongen na te denken over de toekomst en dat heeft geleid tot interessante ideeën over samenwerking. Ik waardeer je inspanningen om $\mathrm{mij}$ in staat te stellen dit project als postdoctoraal onderzoeker voort te zetten.

Ronald, een begeleider zoals jij kan ik iedereen toewensen. Je frisse kijk op mijn onderzoek, niet gehinderd door een vastgeroeste klinische of elektrofysiologische blik, stimuleerde mij vaak om ook eens op een andere manier naar de problemen te kijken. Daarnaast waardeer ik je uitgesproken interesse in mijn persoonlijke welzijn, die nooit verminderde, hoe druk je het ook had met al je verplichtingen. Hoewel politiek volgens jezelf niet je sterkste punt is, koos je in discussies altijd voor mijn welzijn (maar niet noodzakelijk mijn mening). Bij mijn afstuderen bij kennistechnologie boog je je over mijn oma en opa om ze te complimenteren met hun kleinzoon en je nam daarvoor de tijd. Ze stonden helemaal te glimmen.

Dan een speciaal woord van dank voor het hoofd van de afdeling Cardiologie in MUMC+, Harry Crijns, van wie ik altijd veel indirecte steun heb ervaren, met name 
bij onze klinische inspanningen, maar ook in de vorm van een vriendelijk woordje op de gang. Ik kijk er naar uit om ons onderzoek voor klinische doeleinden verder uit te bouwen. Also thanks to the other members of the assessment committee: Olaf Dössel, Elia Formisano, Yoram Rudy, Hein Wellens, and Joachim Wildberger.

Onderzoek is allang geen one-man-job meer en veel van dit werk was niet mogelijk geweest door de directe of indirecte steun van een grote groep mensen. De eerste scanprotocollen die ik voor mijn onderzoek gebruikte zijn nog ondersteund door Andreas Herrler en zijn collega's van het anatomie departement. Vanuit de radiologie is altijd steun geweest van Joachim Wildberger, Marco Das, Ralph Eschweiler, Ankie Hersbach en in een dubbelrol Bas Kietselaer. Daarnaast dank aan Frits Prinzen voor zijn steun en adviezen. De preklinische experimenten waren nooit mogelijk geweest zonder de enthousiaste steun van zijn team: Marc Strik, Lars van Middendorp, Marion Kuiper, Sophie Bosch en Rob Wiegerinck.

Of course I should thank my mathematical and engineering geniuses/colleagues, who contributed considerably to the mathematical work that can be found in this thesis. Joël Karel, af en toe vraag ik me af of wij wel hetzelfde gestudeerd hebben, want jouw wiskundig inzicht overstijgt het mijne verre. Je hebt met je nieuwe ideeën veel bijgedragen aan het werk in deze scriptie. Pietro Bonizzi, if somebody knows something about signal analysis, it's you, and your refreshing viewpoints have had considerable influence as well. Having you as a roommate meant that I could always ask your opinion on something, by which you have influenced also many smaller parts of this thesis. Apart from that, I very much enjoyed all the coffees (and aperitivos!) we shared.

Mijn kamergenoten bij cardiologie door de jaren heen verdienen ook veel dank. Jordi Heijman, niet alleen een collega maar ook een vriend. De kwaliteit van je werk werd voor mij gelukkig eerder een inspiratie dan een onhaalbare eis. Ik ben bijzonder blij dat we onze onderzoeksvelden in het laatste jaar bij elkaar hebben kunnen brengen, nadat we acht jaar geleden voor het eerst kamergenoten werden. Ook bedankt voor alle razendvlugge feedback op enkele hoofdstukken, en voor het vervullen van de rol als paranimf. Ik wens je veel succes in het nastreven van je mooie onderzoekslijnen, en vooral ook in het leven. Michael Clerx, nog zo'n belichaming van de uitstekende cardiologischewiskundige samenwerking, en de daarbij horende mooie dosis eigenzinnigheid. Altijd in voor een relativerende kop goede koffie. Dank voor het leveren van tekstuele feedback op veel van deze hoofdstukken, en met name dank voor de samenwerking die leidde tot 'ons' hoofdstuk! Veel succes met het afronden van je eigen proefschrift. Annerie Moers, altijd in voor een praatje en met een welkome klinische blik op basaalwetenschappelijke onderwerpen. Geen kamergenoot maar toch altijd op de kamer was Monique de Jong, onze immer vrolijke overbuurvrouw. Menig frustrerende dag werd opgeleukt door jouw 
enthousiasme, en daarnaast heb je belangrijke bijdragen geleverd aan ons validatiewerk. Onze 'winter barbecue' is nog steeds een culinair hoogtepunt waarnaar regelmatig verwezen wordt.

Een speciaal woord van dank aan de cardiologen-elektrofysiologen van de afdeling cardiologie in MUMC+, en dan met name Harry Crijns, Laurent Pison en Rachel ter Bekke. Het selecteren en doorsturen van patiënten voor ons ECGI onderzoek is van onschatbare waarde, en ik kijk ernaar uit om onze samenwerking uit te breiden. Daarnaast ook dank aan de imaging cardiologen, voor het meedenken over de toekomst van ECGI in relatie tot beeldvormende technieken.

De vriendelijkheid en wetenschappelijke interesse van het personeel van het 'elektrofysiologisch onderzoek' (EFO) lab is daarnaast ook van onschatbare waarde gebleken. Behalve van de immer enthousiaste Laurent heb ik veel geleerd van Ruud Duysings, en veel steun ervaren van de andere teamleden.

Also many thanks to all the international contacts and collaborators that I've met over the years. Within the Netherlands, this includes Adriaan van Oosterom, Peter van Dam, Arno Janssen and Peter Oosterhoff. Special thanks to Dana Brooks, Burak Erem and Jaume Coll-Font, for our (award winning) collaboration. I hope to extend our collaboration further! Rob MacLeod, many thanks for our many fruitful discussions at conferences, for welcoming me at your lab, and for providing me with guidance in general. Special thanks to Jess, Brett, Wilson and Karli for creating a very welcome environment during my visit to the SCI institute in Utah. Een speciaal woord van dank voor Joep Geraedts voor al het carrière-advies onder het genot van goed Belgisch bier.

De teams waar ik onderdeel van uitmaakte zijn groter dan alleen bovenstaande personen en zijn daarnaast ook nog eens veranderlijk over de tijd. Bij cardiologie onder meer dank aan Roel, de immer vriendelijke, altijd behulpzame en alwetende steunpilaar van ons team, Inge, Viola, Miren, Sandrine, Lucien, Cristina, en medepromovendi Dirk, Dan, Jordi, Annerie, Michael, Rachel, Mylène, Pieter, Cristina en Chiara. And at knowledge engineering special thanks to the biomathematics and bioinformatics group, with Enno, Pieter, Zita, Rachel, Evgueni, Carlo, Maryam, Markos, Nasser, Firat, and many others. Daarnaast een woord van dank aan mijn trouwe conferentie-buddy Stef voor alle gezelligheid en het vinden van al die culinaire hoogstandjes over de hele wereld. Ook bedankt aan de 'andere buurmannen' Arne en Theo.

De vele medebestuursleden, commissiegenootjes, klimmaatjes en vrienden van de studenten alpen club MaasSAC hebben mij welkome afleiding geboden tijdens mijn studie en onderzoek. Met name de heerlijke weekenden, zomers en winters in de alpen met Irene, Joris, Marleen, Daniel, Renée, Sjoerd, Rianne, Maaike, Rik, Mark, Marijn, Tomas, Oliver, Gusta, Hanneke, Sytze, Daphne, Chris, Michael, Bram, Yon en vele 


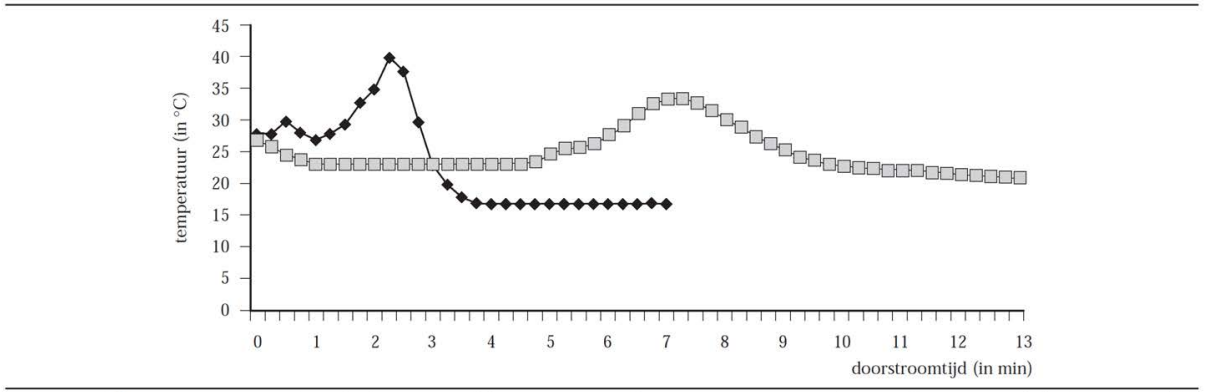

FIGUUR 2. Het temperatuurverloop in het waterleidingsysteem op de gang van een met Legionella pneumophila besmette afdeling van een revalidatiekliniek, bij het maximaal openen van 2 koudwaterkranen na 24 h stilstand in het watersysteem: ( $)$ kraan aan het begin van de gang vlakbij de verticale schacht, waarin ook heetwaterleidingen en verwarmingspijpen liepen; ( $\square$ ) kraan aan het einde van de gang.

Figuur 11.1: Mijn eerste wetenschap

anderen waren fantastisch! Inmiddels uitwaaierend over heel Nederland, maar met een blijvend gedeelde passie, zullen we elkaar hopelijk nog regelmatig treffen in de rotsen of op toppen van bergen, of gewoon voor een goed glas witbier.

Mijn trouwe gang van de middelbare school biedt een welkome tegenhanger voor alle sportieve activiteit. Mischa, Ivo, Frank en Piet, laten we nog lang doorgaan met onze jaarlijkse weekendjes! Mischa, daarnaast veel dank voor alle gezelligheid en je heerlijke kookkunsten tijdens onze man cave periode. Ook bedankt voor alle chats. Maar goed dat onze bazen niet weten hoeveel wij gedurende de dag communiceren, al ben ik ervan overtuigd dat ons gezamenlijk relativerend vermogen alleen maar productief werkt. Daarnaast dank voor het vervullen van de rol als paranimf.

Mijn familie, en dan vooral mijn broertjes en ouders, ben ik ook erg dankbaar. Daan, niet alleen voor alle tijd die we vroeger al schietend, klauterend of knutselend in de bossen doorbrachten, maar ook voor je nuchtere en relativerende rake opmerkingen. Je enorme doorzettingsvermogen was een leidend voorbeeld tijdens sommige onderzoeksfrustraties. Caspar, altijd geïnteresseerd en gelukkig tegenwoordig ook altijd lief. We hebben zelfs even onderzoek met dezelfde methoden gedaan en ook als dat niet het geval was, kon je altijd prima meepraten. Dankzij je brede interesse konden we gelukkig ook over veel andere dingen praten tijdens onze gezellige boulderavonden. En natuurlijk papa en mama, die mij niet alleen letterlijk gevoed hebben, maar ieder op hun eigen manier ook het wetenschappelijk zaadje in mij. Ik herinner me nog levendig hoe ik vroeger als kind met papa door de gang van een revalidatiecentrum mocht rennen, om te meten hoeveel bacteriën er in het water zaten, zie Figuur 11.1. Dit leidde tot mijn eerste wetenschap- 
pelijke publicatie, al word ik daar niet in genoemd... [187] Papa bracht ons alle drie aan de keukentafel zijn passie voor de fysica bij, en dat heeft zeker de keuze voor dit onderzoek beïnvloed. Later, als puber, mocht ik mijn computerkennis inzetten om mama's eerste onderzoeksdatabase op te zetten. Wat was ik trots toen haar inspanningen, vele jaren later, tot haar succesvolle promotie leidden, waarbij ik paranimf mocht zijn. Natuurlijk hebben mijn ouders mij ook buiten de wetenschap veel meegegeven, zoals compassie, empathie en zelfstandigheid, maar ook een voorliefde voor vakanties en tripjes...

En dan natuurlijk nog mijn lieve Irene. Wat ben ik blij dat we zoveel delen: hobby's, opleiding, en een gezond verlangen naar vakanties. Gedurende het laatste jaar accepteerde je dat mijn 'ik werk niet in het weekend' houding langzaam maar zeker moest wijken voor alle deadlines. Het hielp daarbij wellicht dat jij van ons tweeën de enige echte dokter bent, en ik je diensten en lange dagen daarom gemakkelijk kon aangrijpen om zelf nog wat werk te verrichten. Je bewoog mij om als echte Limburger toch naar Brabant te verhuizen (waardoor ik, paradoxaal genoeg, juist een 'trein-Limbo' geworden ben), en nu heb ik zelfs een PSV sjaal. Onze avonturen in de rotsen, bergen en verre oorden zijn hopelijk een metafoor voor alle hoogtepunten die we samen nog gaan vieren in het leven. 
Matthijs Cluitmans was born on December 26, 1985 in Geleen, the Netherlands. After attending high school in Maastricht he went to Leuven (Belgium) to study Physics. After his romanticized idea of physics was carefully crushed by mathematical abstraction, he decided to switch to Medicine and started studying at Maastricht University. To satisfy his still lingering interest in exact sciences, he combined his bachelor study in Medicine with a bachelor study in Knowledge Engineering (a study that mixes computer science, artificial intelligence and applied mathematics). After obtaining both bachelor degrees (in 2008 and 2009), he obtained the Operations Research master degree (cum laude) at the Department of Knowledge Engineer-

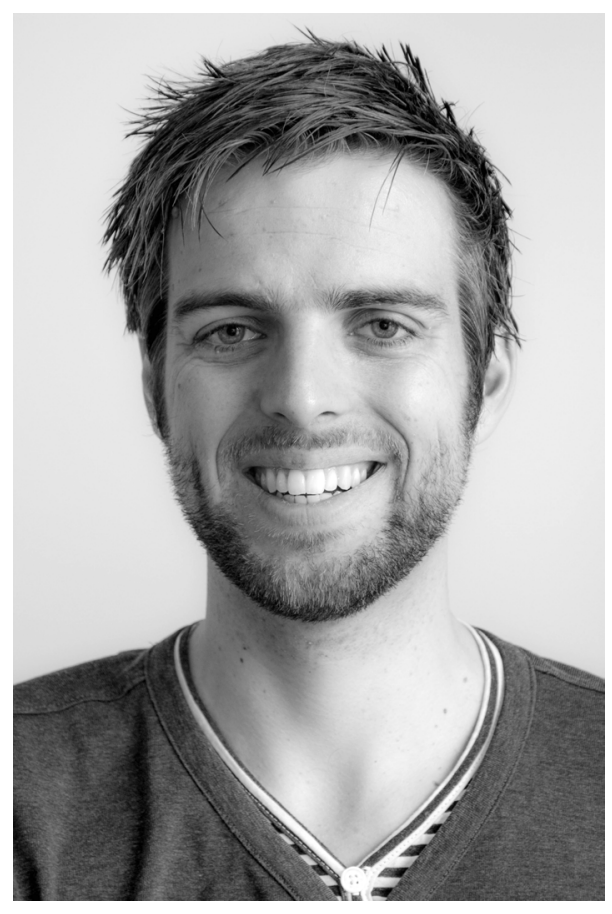
ing in 2010. He then began this combined $\mathrm{MD} / \mathrm{PhD}$ journey, successfully finishing his rotations and obtaining his degree as medical doctor in 2014, and obtaining his $\mathrm{PhD}$ degree in 2016.

In his $\mathrm{PhD}$ research, Matthijs combined mathematical, engineering and clinical approaches to obtain more insight in cardiovascular imaging techniques and the mechanisms behind cardiac arrhythmias. He established various international collaborations, paid a short visit to the University of Utah (Salt Lake City, Utah, USA), played a role in the international Consortium for ECG Imaging, and obtained several awards. During his future career, Matthijs aims to use his dual background as biomedical engineer and medical doctor to bring the fields of engineering and medicine closer together.

Matthijs lives in Eindhoven, the Netherlands, and enjoys mountaineering, mountain biking, photography, and drinking a beer with friends. He has a special interest in mountain medicine and volunteers as an MD during sports events in south Limburg (despite its lack of mountains). 


\section{AWARDS AND FELLOWSHIPS}

\section{Clinical Needs Translational Award (winner)}

Winner of the joint Clinical Needs Translational Award, a joint initiative between the Working Group on e-Cardiology of the European Society of Cardiology (ESC) and Computing in Cardiology (CinC). Awarded at the CinC conference 2016, Vancouver, Canada.

\section{IBBM Fellowship}

Awarded a fellowship for the Summer Course on Image-based Biomedical Modeling (IBBM), 2016, Park City, Utah, USA.

\section{Young Investigator Award (finalist)}

Finalist in the Jos Willems Young Investigator Competition of the International Society for Computerized Electrocardiology (ISCE) conference 2015, San Jose, CA, USA.

\section{Young Investigator Award (winner)}

Winner of the Rosanna Degani Young Investigator Awards for the Computing in Cardiology (CinC) conference 2014, Cambridge, MA, USA.

\section{Best poster presentation}

Awarded a best poster prize at the Computing in Cardiology conference 2013, Zaragoza, Spain.

\section{Publications}

\section{JOURNAL ARTICLES}

1. M Cluitmans, P Bonizzi, J Karel, M Das, B Kietselaer, M De Jong, F Prinzen, R Peeters, R Westra, and P Volders, "In vivo validation of electrocardiographic imaging," 2016. In revision.

2. M Cluitmans*, J Karel*, P Bonizzi, P Volders, R Westra, R Peeters, "Electrocardiographic imaging: regularization with spatiotemporal sparsity in the wavelet domain," 2016 . Submitted.

3. M Cluitmans*, M Clerx*, N Vandersickel, R Peeters, P Volders, and R Westra, "Physiologybased regularization of the electrocardiographic inverse problem," 2016. In revision.

4. T Lankveld, S Zeemering, E Marcos, M Rienstra, M Potse, H Brunner-La Rocca, M Cluitmans, P Volders, P Dagnelie, R Henry, M Schram, S Sep, C Stehouwer, I Van Gelder, H Crijns, and U Schotten, "Increased p-wave complexity in patients with atrial fibrillation compared to a control population," 2016. Submitted.

5. M Cluitmans, R Peeters, R Westra, and P Volders, "Noninvasive reconstruction of cardiac electrical activity: update on current methods, applications and challenges.," Neth Heart J, vol. 23 , no. 6, pp. 301-311, 2015 .

* These first two authors contributed equally to this work.

\section{CONFERENCE PROCEEDINGS}

1. M Cluitmans, J Coll-Font, B Erem, D Brooks, P Bonizzi, J Karel, P Volders, R Peeters, and $\mathrm{R}$ Westra, "Spatiotemporal activation time estimation improves noninvasive localization of cardiac electrical activity," in Computing in Cardiology, vol. 43, 2016.

2. M Cluitmans, J Karel, P Bonizzi, M Jong, P Volders, R Peeters, and R Westra, "In-vivo evaluation of reduced-lead-systems in noninvasive reconstruction and localization of cardiac electrical activity," in Computing in Cardiology, vol. 42, 2015, pp. $221-4$. 
3. M Cluitmans, $M$ Jong, $P$ Volders, $R$ Peeters, and R Westra, "Physiology-based regularization improves noninvasive reconstruction and localization of cardiac electrical activity," in Computing in Cardiology, vol. 41, 2014, pp. 1-4.

4. M Cluitmans, P Bonizzi, J Karel, P Volders, R Peeters, and R Westra, "Inverse reconstruction of epicardial potentials improves by vectorcardiography and realistic potentials," in Computing in Cardiology, vol. 40, 2013, pp. 369-372.

5. M Cluitmans, J Karel, P Bonizzi, P Volders, R Westra, and R Peeters, "Wavelet-sparsity based regularization over time in the inverse problem of electrocardiography," in Conf Proc IEEE Eng Med Biol Soc., IEEE, 2013, pp. 3781-3784.

6. M Cluitmans, R Peeters, $P$ Volders, and R Westra, "Realistic training data improve noninvasive reconstruction of heart-surface potentials," in Conf Proc IEEE Eng Med Biol Soc., IEEE, 2012, pp. 6373-6376.

\section{AbSTRACTS}

1. M Cluitmans, M Clerx, J Karel, P Bonizzi, R Peeters, P Volders, and R Westra, "Whole-heart simulations improve localization of origin of a heart beat in noninvasive electrocardiographic imaging," in International Conference on Basic and Clinical Multimodal Imaging, 1 oth International Symposium on Noninvasive Functional Source Imaging, 2015.

2. M Cluitmans, M Jong, J Karel, P Bonizzi, F Prinzen, R Peeters, R Westra, and P Volders, "High-resolution validation of localization of electrocardiographic imaging shows a centimeter-accuracy," in International Society for Computerized Electrocardiology (ISCE) 40th Annual Conference, San Jose, California, USA, 15-19 April 2015, 2015.

3. M Cluitmans, J Karel, P Bonizzi, P Volders, R Peeters, and R Westra, "Wavelet-sparsity based regularization," in ECG Imaging 2015 Workshop, Bad Herrenalb, Germany, 25-28 March 2015, 2015 .

4. M Cluitmans, and P Volders, "Noninvasive imaging of repolarization gives insight in arrhythmogenic substrate," in Nederlandse Vereniging voor Cardiologie (NVVC) Voorjaarscongres, Noordwijk, the Netherlands, 9-10 April 2015, 2015.

5. M Cluitmans, P Bonizzi, J Karel, R Peeters, P Volders, and R Westra, "Inverse reconstruction of epicardial potentials is improved by novel regularization methods and extensive validaton," in Workshop 3: Integrating Modalities and Scales in Life Science Imaging, Columbus, Ohio, USA, 17- 21 March 2014, Mathematical Biosciences Institute (MBI), Ed., Monica Hurdal, Michael Liebling, Rob MacLeod, and Kristin Swanson, 2014.

6. R Ter Bekke, A Moers, M Jong, M Cluitmans, E Vanoli, and P Volders, "Left-stellate ganglion stimulation triggers torsades de pointes in a canine model of drug-induced long-qt 1 syndrome," in Heart Rhythm, Heart Rhythm Society, 2013.

7. M Cluitmans, R Westra, and R Peeters, "Singular value decomposition and change of basis improve the inverse solution in electrocardiography," in 3rd Dutch Conference on BioMedical Engineering (BME 2011), Egmond aan Zee, 20-21 Jan. 2011, 2011.

8. M Cluitmans, R Westra, and R Peeters, "The inverse solution of electrocardiography is improved by including localized, physiological knowledge," in 3oth Benelux Meeting on System and Control, Lommel, Belgium, 15-17 March 2011, ISBN 978-90-9026089-1, Universiteit Gent, 2011 , p. 197. 


\section{Figure acknowledgements}

Figure 1.1 (page 3): From [1], with permission.

Figure 1.3 (page 5) : From Servier Medical Art by Servier (http://www.servier.com, licensed under a Creative Commons Attribution 3.0 Unported License) and Textbook of Cardiology (www.textbookofcardiology.org, licensed under the Creative Commons AttributionNonCommercial-ShareAlike 3.0 Unported License).

Figure 1.4 (page 7) : From: [188] (top) and [189] (bottom), with permission.

Figure 1.5 (page 9): From [8], public domain.

Figure 1.6 (page 10): From [10], public domain.

Figure 1.7 (page 11) : From https://en.wikipedia.org/wiki/Electrocardiography\# /media/File:Willem_Einthoven_ECG.jpg, public domain.

Figure 7.3 (page 116) : Adapted from Wikimedia Commons, File:Wiggers Diagram.png, CC BY-SA 2.5 .

All other figures are created by the author of this thesis, of by coauthors of the chapters. 


\section{Bibliography}

[1] Durrer D, Dam RT van, Freud GE, Janse MJ, Meijler FL, and Arzbaecher RC, “Total excitation of the isolated human heart," Circulation, vol. 41, no. 6, pp. 899-912, 1970.

[2] Thomson Reuters. (2016). Web of science, [Online]. Available: http : / / webofknowledge.com.

[3] Issa ZF, Miller JM, and Zipes DP, "Chapter 1 - molecular mechanisms of cardiac electrical activity," in Clinical Arrhythmology and Electrophysiology: A Companion to Braunwald's Heart Disease (Second Edition), Issa ZF, Miller JM, and Zipes DP, Eds., Second Edition, Philadelphia: W.B. Saunders, 2012, pp. 1 -9.

[4] Issa ZF, Miller JM, and Zipes DP, "Chapter 3 - electrophysiological mechanisms of cardiac arrhythmias," in Clinical Arrhythmology and Electrophysiology: A Companion to Braunwald's Heart Disease (Second Edition), Issa ZF, Miller JM, and Zipes DP, Eds., Second Edition, Philadelphia: W.B. Saunders, 2012 , pp. 36-61.

[5] Jenkins D. (2009). A (not so) brief history of electrocardiography, [Online]. Available: http://www . ecglibrary. com/ecghist.html.

[6] Galvani L, "De viribus electricitatis in motu musculari commentarius," De Bononiensi Scientiarum et Artium Instituto atque Academia Commentarii, vol. 7, pp. 363-419, 1791.

[7] Matteucci C, "Sur un phenomene physiologique produit par les muscles en contraction," Ann Chim Phys, vol. 6, no. 1842, pp. 339-341, 1842.

[8] Waller $\mathrm{AD}$, "A demonstration on man of electromotive changes accompanying the heart's beat," J Physiol, vol. 8, no. 5, pp. 229-234, 1887.

[9] Einthoven W, "Nieuwe methoden voor clinisch onderzoek [new methods for clinical investigation]," Ned T Geneesk, vol. 29, no. II, pp. 263-286, 1893.

[10] Einthoven W, "Ueber die form des menschlichen electrocardiogramms," Pflügers Archiv European Journal of Physiology, vol. 60, no. 3, pp. 101-123, 1895 .

[11] Einthoven W, "Le telecardiogramme," Arch Int de Physiol, vol. 4, pp. 132-164, 1906.

[12] Barnes AR, Pardee HE, White PD, Wilson FN, Wolferth CC, et al., "Standardization of precordial leads: supplementary report," American Heart Journal, vol. 15, no. 2, pp. 235-239, 1938.

[13] Ambroggi $\mathrm{L}$ de and Corlan $\mathrm{AD}$, "Body surface potential mapping," in Comprehensive Electrocardiology, Macfarlane PW, Oosterom A van, Pahlm O, Kligfield P, Janse M, and Camm J, Eds., Springer London, 2010, pp. 1375-1413.

[14] Rudy Y and Messinger-Rapport BJ, "The inverse problem in electrocardiography: solutions in terms of epicardial potentials," Crit Rev Biomed Eng, vol. 16, no. 3, pp. 21 5-68, 1988.

[15] Dam PM van, Oostendorp TF, Linnenbank AC, and Oosterom A van, "Non-invasive imaging of cardiac activation and recovery," Ann Biomed Eng, vol. 37, no. 9, pp. 1739-1756, 2009. 
[16] Van Oosterom A, "The equivalent double layer: source models for repolarization," in Comprehensive Electrocardiology, Macfarlane PW, Oosterom A van, Pahlm O, Kligfield P, Janse $\mathrm{M}$, and Camm J, Eds., Springer London, 2010, pp. 227-246.

[17] Cluitmans M, Electrocardiographic imaging as a medical tool: the mathematical basis and medical implications of electrocardiographic imaging. Bachelor thesis, 2007.

[18] Scientific Computing and Imaging Institute (SCI), CIBC, $\mathrm{Seg}_{3} D$ : volumetric image segmentation and visualization. 2015.

[19] Burton BM, Tate JD, Erem B, Swenson DJ, Wang DF, Steffen M, Brooks DH, Dam PM van, and Macleod RS, "A toolkit for forward/inverse problems in electrocardiography within the SCIRun problem solving environment," Conf Proc IEEE Eng Med Biol Soc, vol. 2011, pp. 267-70, 2011.

[20] Hansen P, "Regularization tools version 4.0 for matlab 7.3," Numerical Algorithms, vol. 46, no. 2, pp. 189-194, 2007.

[21] MATLAB, version 8.2.o.701 (R2013b). Natick, Massachusetts, USA: The MathWorks Inc., 2013.

[22] Martin RO and Pilkington TC, "Unconstrained inverse electrocardiography: epicardial potentials," Biomedical Engineering, IEEE Transactions on, vol. BME-19, no. 4, pp. 276-285, 1972.

[23] Martin RO, Pilkington TC, and Morrow MN, "Statistically constrained inverse electrocardiography," IEEE Trans Biomed Eng, vol. 22, no. 6, pp. 487-492, 1975.

[24] Barr RC, Ramsey M, and Spach MS, "Relating epicardial to body surface potential distributions by means of transfer coefficients based on geometry measurements," IEEE Trans Biomed Eng, vol. 24, no. 1, pp. 1-11, 1977.

[25] Franzone PC, Taccardi B, and Viganotti C, "An approach to inverse calculation of epicardial potentials from body surface maps," Adv Cardiol, vol. 21, pp. 50-4, 1978.

[26] Yamashita $\mathrm{Y}$ and Takahashi T, "Use of the finite element method to determine epicardial from body surface potentials under a realistic torso model," IEEE Trans Biomed Eng, vol. 31 , no. 9, pp. 611-621, 1984 .

[27] Cuppen JJ and Oosterom A van, "Model studies with the inversely calculated isochrones of ventricular depolarization," IEEE Trans Biomed Eng, vol. 31, no. 10, pp. 652-659, 1984.

[28] Messinger-Rapport BJ and Rudy Y, “The inverse problem in electrocardiography: a model study of the effects of geometry and conductivity parameters on the reconstruction of epicardial potentials," IEEE Trans Biomed Eng, vol. 33, no. 7, pp. 667-676, 1986.

[29] Messinger-Rapport BJ and Rudy Y, "Regularization of the inverse problem in electrocardiography: a model study," Mathematical Biosciences, vol. 89, no. 1, pp. 79-118, 1988.

[30] Gulrajani RM, Savard P, and Roberge FA, “The inverse problem in electrocardiography: solutions in terms of equivalent sources," Crit Rev Biomed Eng, vol. 16, no. 3, pp. 171-214, 1988.

[31 ] Barr RC and Spach MS, "Inverse calculation of qrs-t epicardial potentials from body surface potential distributions for normal and ectopic beats in the intact dog," Circ Res, vol. 42, no. 5, pp. 661-675, 1978.

[32] Messinger-Rapport BJ and Rudy Y, "Noninvasive recovery of epicardial potentials in a realistic heart-torso geometry. normal sinus rhythm," Circ Res, vol. 66, no. 4, pp. 1023-39, 1990.

[33] Oster HS, Taccardi B, Lux RL, Ershler PR, and Rudy Y, "Noninvasive electrocardiographic imaging: reconstruction of epicardial potentials, electrograms, and isochrones and localiza- 
tion of single and multiple electrocardiac events," Circulation, vol. 96, no. 3, pp. 1012-24, 1997.

[34] Ramanathan C, Ghanem RN, Jia P, Ryu K, and Rudy Y, "Noninvasive electrocardiographic imaging for cardiac electrophysiology and arrhythmia," Nat Med, vol. 10, no. 4, pp. 422-8, 2004 .

[35] Sapp JL, Dawoud F, Clements JC, and Horácek BM, "Inverse solution mapping of epicardial potentials: quantitative comparison with epicardial contact mapping," Circ Arrhythm Electrophysiol, vol. 5, no. 5, pp. 1001-1009, 2012.

[36] MacLeod RS and Brooks DH, "Recent progress in inverse problems in electrocardiology," IEEE Eng Med Biol Mag, vol. 17, no. 1, pp. 73-83, 1998.

[37] Rudy Y, "Noninvasive electrocardiographic imaging of arrhythmogenic substrates in humans," Circ Res, vol. 112, no. 5, pp. 863-874, 2013.

[38] Oosterom A van, “The inverse problem of bioelectricity: an evaluation," Med Biol Eng Comput, vol. 50, no. 9, pp. 891-902, 2012.

[39] Van der Graaf AM, Bhagirath P, Ramanna H, Driel VJ van, Hooge J de, Groot NM de, and Götte MJ, "Noninvasive imaging of cardiac excitation: current status and future perspective," Annals of Noninvasive Electrocardiology, vol. 19, no. 2, pp. 105-113, 2014.

[40] Van Oosterom A, "A comparison of electrocardiographic imaging based on two source types," Europace, vol. 16 Suppl 4, pp. iv120-iv128, 2014.

[41] Oostendorp TF, Dessel PFHM van, Coronel R, Belterman C, Linnenbank AC, Schie IH van, Oosterom A van, Oosterhoff P, Dam PM van, and Bakker JMT de, "Noninvasive detection of epicardial and endocardial activity of the heart," Neth Heart J, vol. 19, no. 11, pp. 488491, 2011.

[42] Ramanathan C, Jia P, Ghanem R, Ryu K, and Rudy Y, "Activation and repolarization of the normal human heart under complete physiological conditions," Proc Natl Acad Sci U S A, vol. 103, no. 16, pp. 6309-14, 2006.

[43] Rudy Y, "Cardiac repolarization: insights from mathematical modeling and electrocardiographic imaging (ecgi)," Heart Rhythm, vol. 6, no. 11, Supplement 1, S49-S55, 2009.

[44] Cuculich PS, Wang Y, Lindsay BD, Faddis MN, Schuessler RB, Damiano RJ, Li L, and Rudy $\mathrm{Y}$, "Noninvasive characterization of epicardial activation in humans with diverse atrial fibrillation patterns," Circulation, vol. 122, no. 14, pp. 1364-1372, 2010.

[45] Wang Y, Cuculich PS, Zhang J, Desouza KA, Vijayakumar R, Chen J, Faddis MN, Lindsay BD, Smith TW, and Rudy Y, "Noninvasive electroanatomic mapping of human ventricular arrhythmias with electrocardiographic imaging," Sci Transl Med, vol. 3, no. 98, 98ra84, 2011.

[46] Cuculich PS, Zhang J, Wang Y, Desouza KA, Vijayakumar R, Woodard PK, and Rudy Y, "The electrophysiological cardiac ventricular substrate in patients after myocardial infarction: noninvasive characterization with electrocardiographic imaging," J Am Coll Cardiol, vol. 58, no. 18, pp. 1893-1902, 2011.

[47] Marrus SB, Andrews CM, Cooper DH, Faddis MN, and Rudy Y, "Repolarization changes underlying long-term cardiac memory due to right ventricular pacing: noninvasive mapping with ecgi," Circ Arrhythm Electrophysiol, vol. 5, pp. 773-781, 2012.

[48] Zhang J, Desouza KA, Cuculich PS, Cooper DH, Chen J, and Rudy Y, "Continuous ecgi mapping of spontaneous vt initiation, continuation, and termination with antitachycardia pacing," Heart Rhythm, vol. 10, no. 8, pp. 1244-5, 2012.

[49] van der Graaf AWM, Bhagirath P, van Driel VJHM, Ramanna H, de Hooge J, de Groot NMS, and Götte MJW, "Computing volume potentials for noninvasive imaging of cardiac excitation," Ann Noninvasive Electrocardiol, vol. 20, no. 2, pp. 132-139, 2014. 
[50] Huiskamp GJ and van Oosterom A, "The depolarization sequence of the human heart surface computed from measured body surface potentials." IEEE Trans Biomed Eng, vol. 35, no. 12, pp. 1047-1058, 1988.

[51] Oosterom A van and Huiskamp GJ, "The effect of torso inhomogeneities on body surface potentials quantified using "tailored"geometry.," J Electrocardiol, vol. 22, no. 1, pp. 53-72, 1989.

[52] Dam PM van, Oostendorp TF, and Oosterom A van, "Application of the fastest route algorithm in the interactive simulation of the effect of local ischemia on the ecg," Med Biol Eng Comput, vol. 47, no. 1, pp. 11-20, 2009.

[53] Dam PM van, Tung R, Shivkumar K, and Laks M, "Quantitative localization of premature ventricular contractions using myocardial activation ECGI from the standard 12-lead electrocardiogram," Journal of Electrocardiology, vol. 46, no. 6, pp. 574-9, 2013.

[54] Ploux S, Lumens J, Whinnett Z, Montaudon M, Strom M, Ramanathan C, Derval N, Zemmoura A, Denis A, De Guillebon M, Shah A, Hocini M, Jaïs P, Ritter P, Haïssaguerre M, Wilkoff $\mathrm{BL}$, and Bordachar $\mathrm{P}$, "Noninvasive electrocardiographic mapping to improve patient selection for cardiac resynchronization therapy: beyond qrs duration and left bundle branch block morphology," J Am Coll Cardiol, vol. 61, no. 24, pp. 2435-2443, 2013.

[55] Berger T, Pfeifer B, Hanser FF, Hintringer F, Fischer G, Netzer M, Trieb T, Stuehlinger M, Dichtl W, Baumgartner C, Pachinger O, and Seger M, "Single-beat noninvasive imaging of ventricular endocardial and epicardial activation in patients undergoing crt," PLOS ONE, vol. 6, no. 1, e16255, Jan. 2011.

[56] Han C, Liu Z, Zhang X, Pogwizd S, and He B, "Noninvasive three-dimensional cardiac activation imaging from body surface potential maps: a computational and experimental study on a rabbit model," IEEE Trans Med Imaging, vol. 27, no. 11, pp. 1622-30, 2008.

[57] Revishvili AS, Wissner E, Lebedev DS, Lemes C, Deiss S, Metzner A, Kalinin VV, Sopov OV, Labartkava EZ, Kalinin AV, Chmelevsky M, Zubarev SV, Chaykovskaya MK, Tsiklauri MG, and Kuck KH, "Validation of the mapping accuracy of a novel non-invasive epicardial and endocardial electrophysiology system," Europace, vol. 17, no. 8, pp. 1282-1288, 2015.

[58] Cakulev I, Sahadevan J, Arruda M, Goldstein RN, Hong M, Intini A, Mackall JA, Stambler BS, Ramanathan C, Jia P, Strom M, and Waldo AL, "Confirmation of novel noninvasive high-density electrocardiographic mapping with electrophysiology study: implications for therapy," Circ Arrhythm Electrophysiol, vol. 6, no. 1, pp. 68-75, 2013.

[59] Intini A, Goldstein RN, Jia P, Ramanathan C, Ryu K, Giannattasio B, Gilkeson R, Stambler BS, Brugada P, Stevenson WG, Rudy Y, and Waldo AL, "Electrocardiographic imaging (ecgi), a novel diagnostic modality used for mapping of focal left ventricular tachycardia in a young athlete," Heart Rhythm, vol. 2, no. 11, pp. 1250-2, 2005.

[60] Burnes JE, Taccardi B, Ershler PR, and Rudy Y, "Noninvasive electrocardiogram imaging of substrate and intramural ventricular tachycardia in infarcted hearts," J Am Coll Cardiol, vol. 38, no. 7, pp. 2071-2078, 2001.

[61] Han C, Pogwizd SM, Killingsworth CR, and He B, "Noninvasive imaging of threedimensional cardiac activation sequence during pacing and ventricular tachycardia," Heart Rhythm, vol. 8, no. 8, pp. 1266-1272, 2011.

[62] Shah AJ, Hocini M, Xhaet O, Pascale P, Roten L, Wilton SB, Linton N, Scherr D, Miyazaki S, Jadidi AS, Liu X, Forclaz A, Nault I, Rivard L, Pedersen ME, Derval N, Sacher F, Knecht S, Jais P, Dubois R, Eliautou S, Bokan R, Strom M, Ramanathan C, Cakulev I, Sahadevan J, Lindsay B, Waldo AL, and Haissaguerre M, "Validation of novel $3 \mathrm{~d}$ electrocardiographic 
mapping of atrial tachycardias by invasive mapping and ablation: a multicenter study," $\mathrm{JAm}$ Coll Cardiol, vol. 62, no. 10, pp. 889-97, 2013.

[63] Wang Y, Cuculich PS, Woodard PK, Lindsay BD, and Rudy Y, "Focal atrial tachycardia after pulmonary vein isolation: noninvasive mapping with electrocardiographic imaging (ecgi)," Heart Rhythm, vol. 4, no. 8, pp. 1081-1084, 2007.

[64] Roten L, Pedersen M, Pascale P, Shah A, Eliautou S, Scherr D, Sacher F, and Haïssaguerre $\mathrm{M}$, "Noninvasive electrocardiographic mapping for prediction of tachycardia mechanism and origin of atrial tachycardia following bilateral pulmonary transplantation," J Cardiovasc Electrophysiol, vol. 23, no. 5, pp. 553-555, 2012.

[65] Title LM, Iles SE, Gardner MJ, Penney CJ, Clements JC, and Horácek BM, "Quantitative assessment of myocardial ischemia by electrocardiographic and scintigraphic imaging," $J$ Electrocardiol, vol. 36 Suppl, pp. 17-26, 2003.

[66] Jia P, Ramanathan C, Ghanem RN, Ryu K, Varma N, and Rudy Y, "Electrocardiographic imaging of cardiac resynchronization therapy in heart failure: observation of variable electrophysiologic responses," Heart Rhythm, vol. 3, no. 3, pp. 296-310, 2006.

[67] Varma N, Jia P, and Rudy Y, "Electrocardiographic imaging of patients with heart failure with left bundle branch block and response to cardiac resynchronization therapy," J Electrocardiol, vol. 40, no. 6 Suppl, S $174-S_{178,} 2007$.

[68] Berger T, Fischer G, Pfeifer B, Modre R, Hanser F, Trieb T, Roithinger FX, Stuehlinger M, Pachinger O, Tilg B, and Hintringer F, "Single-beat noninvasive imaging of cardiac electrophysiology of ventricular pre-excitation," Journal of the American College of Cardiology, vol. 48, no. 10, pp. $2045-2052,2006$.

[69] Ghosh S, Avari JN, Rhee EK, Woodard PK, and Rudy Y, "Noninvasive electrocardiographic imaging (ecgi) of a univentricular heart with wolff-parkinson-white syndrome," Heart Rhythm, vol. 5, no. 4, pp. 605-8, 2008.

[70] Ghosh S, Avari JN, Rhee EK, Woodard PK, and Rudy Y, "Hypertrophic cardiomyopathy with preexcitation: insights from noninvasive electrocardiographic imaging (ecgi) and catheter mapping," J Cardiovasc Electrophysiol, vol. 19, no. 11, pp. 1215-1217, 2008.

[71] Ghanem RN, Burnes JE, Waldo AL, and Rudy Y, "Imaging dispersion of myocardial repolarization, ii: noninvasive reconstruction of epicardial measures," Circulation, vol. 104, no. 11, pp. 1306-12, 2001.

[72] Ghosh S, Cooper DH, Vijayakumar R, Zhang J, Pollak S, Haïssaguerre M, and Rudy Y, "Early repolarization associated with sudden death: insights from noninvasive electrocardiographic imaging," Heart Rhythm, vol. 7, no. 4, pp. 534-537, 2010.

[73] Vijayakumar R, Silva JNA, Desouza KA, Abraham RL, Strom M, Sacher F, Van Hare GF, Haïssaguerre M, Roden DM, and Rudy Y, "Electrophysiologic substrate in congenital long qt syndrome: noninvasive mapping with electrocardiographic imaging (ecgi)," Circulation, vol. 130, no. 22, pp. 1936-43, 2014.

[74] Aliot EM, Stevenson WG, Almendral-Garrote JM, Bogun F, Calkins CH, Delacretaz E, Della Bella P, Hindricks G, Jaïs P, Josephson ME, Kautzner J, Kay GN, Kuck KH, Lerman BB, Marchlinski F, Reddy V, Schalij MJ, Schilling R, Soejima K, Wilber D, European Heart Rhythm Association (EHRA), Registered Branch of the European Society of Cardiology (ESC), Heart Rhythm Society (HRS), American College of Cardiology (ACC), and American Heart Association (AHA), "Ehra/hrs expert consensus on catheter ablation of ventricular arrhythmias," Heart Rhythm, vol. 6, no. 6, pp. 886-933, 2009.

[75] Haissaguerre M, Hocini M, Denis A, Shah AJ, Komatsu Y, Yamashita S, Daly M, Amraoui S, Zellerhoff S, Picat MQ Quotb A, Jesel L, Lim H, Ploux S, Bordachar P, Attuel G, Meillet V, 
Ritter P, Derval N, Sacher F, Bernus O, Cochet H, Jais P, and Dubois R, "Driver domains in persistent atrial fibrillation,” Circulation, vol. 130, no. 7, pp. 530-538, 2014

[76] Adabag AS, Luepker RV, Roger VL, and Gersh BJ, "Sudden cardiac death: epidemiology and risk factors," Nat Rev Cardiol, vol. 7, no. 4, pp. 216-225, 2010.

[77] Burnes JE, Taccardi B, and Rudy Y, "A noninvasive imaging modality for cardiac arrhythmias," Circulation, vol. 102, no. 17, pp. 2152-2158, 2000.

[78] Ghanem RN, Jia P, Ramanathan C, Ryu K, Markowitz A, and Rudy Y, "Noninvasive electrocardiographic imaging (ecgi): comparison to intraoperative mapping in patients," Heart Rhythm, vol. 2, no. 4, pp. 339-54, 2005.

[79] Zorzi A, Migliore F, Elmaghawry M, Silvano M, Marra MP, Niero A, Nguyen K, Rigato I, Bauce B, Basso C, Thiene G, Iliceto S, and Corrado D, "Electrocardiographic predictors of electroanatomic scar size in arrhythmogenic right ventricular cardiomyopathy: implications for arrhythmic risk stratification," J Cardiovasc Electrophysiol, vol. 24, no. 12, pp. 1321-1327, 2013.

[80] Jiang Y, Qian C, Hanna R, Farina D, and Dössel O, "Optimization of the electrode positions of multichannel ecg for the reconstruction of ischemic areas by solving the inverse electrocardiographic problem," International Journal of Bioelectromagnetism, vol. 11, no. 1, pp. 2737, 2009 .

[81] Messnarz B, Seger M, Modre R, Fischer G, Hanser F, and Tilg B, "A comparison of noninvasive reconstruction of epicardial versus transmembrane potentials in consideration of the null space," IEEE Trans Biomed Eng, vol. 51, no. 9, pp. 1609-1618, 2004.

[82] Pullan AJ, Cheng LK, Nash MP, Ghodrati A, MacLeod R, and Brooks DH, “The inverse problem of electrocardiography," in Comprehensive Electrocardiology, Macfarlane PW, Oosterom A van, Pahlm O, Kligfield P, Janse M, and Camm J, Eds., Springer London, 2010, pp. 299-344.

[83] Modre R, Tilg B, Fischer G, and Wach P, "Noninvasive myocardial activation time imaging: a novel inverse algorithm applied to clinical ecg mapping data," IEEE Trans Biomed Eng, vol. 49, no. 10, pp. 1153-1161, 2002.

[84] Nielsen BF, Lysaker M, and Tveito A, "On the use of the resting potential and level set methods for identifying ischemic heart disease: an inverse problem," Journal of Computational Physics, vol. 220, no. 2, pp. 772 -790, 2007.

[85] Potse M, Dubé B, Richer J, Vinet A, and Gulrajani RM, "A comparison of monodomain and bidomain reaction-diffusion models for action potential propagation in the human heart," Biomedical Engineering, IEEE Transactions on, vol. 53, no. 12, pp. 2425-2435, 2006.

[86] Nielsen BF, Cai X, and Lysaker M, "On the possibility for computing the transmembrane potential in the heart with a one shot method: an inverse problem," Mathematical Biosciences, vol. 210 , no. 2, pp. $523-553,2007$.

[87] Wang D, Kirby RM, Macleod RS, and Johnson CR, "Inverse electrocardiographic source localization of ischemia: an optimization framework and finite element solution," J Comput Phys, vol. 250, pp. 403-424, 2013.

[88] Stenroos M, "The transfer matrix for epicardial potential in a piece-wise homogeneous thorax model: the boundary element formulation,” Phys Med Biol, vol. 54, no. 18, pp. 5443-55, 2009.

[89] Stenroos M and Haueisen J, "Boundary element computations in the forward and inverse problems of electrocardiography: comparison of collocation and galerkin weightings," IEEE Trans Biomed Eng, vol. 55, no. 9, pp. 2124-33, 2008. 
[90] Ramanathan C and Rudy Y, "Electrocardiographic imaging: ii. effect of torso inhomogeneities on noninvasive reconstruction of epicardial potentials, electrograms, and isochrones," J Cardiovasc Electrophysiol, vol. 12, no. 2, pp. 241-252, 2001.

[91] MacLeod RS and Brooks DH, "Recent progress in inverse problems in electrocardiology," IEEE Eng Med Biol Mag, vol. 17, no. 1, pp. 73-83, 1998.

[92] Hansen PC and O'Leary DP, “The use of the 1-curve in the regularization of discrete illposed problems," SIAM Journal on Scientific Computing, vol. 14, no. 6, pp. 1487-1503, 1993.

[93] Tikhonov A and Arsenin V, Solutions of ill-posed problems, ser. Scripta series in mathematics. Washington: Winston, 1977.

[94] Golub G and Reinsch C, "Singular value decomposition and least squares solutions," Numerische Mathematik, vol. 14, pp. 403-420, 51970.

[95] Greensite F and Huiskamp G, "An improved method for estimating epicardial potentials from the body surface," Biomedical Engineering, IEEE Transactions on, vol. 45, no. 1, pp. 98 104, 1998.

[96] Ramanathan C, Jia P, Ghanem R, Calvetti D, and Rudy Y, "Noninvasive electrocardiographic imaging (ecgi): application of the generalized minimal residual (gmres) method," Ann Biomed Eng, vol. 31, no. 8, pp. 981-94, 2003.

[97] Cluitmans M, Peeters R, Volders P, and Westra R, "Realistic training data improve noninvasive reconstruction of heart-surface potentials," in Conf Proc IEEE Eng Med Biol Soc., IEEE, 2012, pp. 6373-6376.

[98] Milanič M, Jazbinšek V, Macleod RS, Brooks DH, and Hren R, "Assessment of regularization techniques for electrocardiographic imaging," J Electrocardiol, vol. 47, no. 1, pp. 20-8, 2014.

[99] Cluitmans MJM, Peeters RLM, Westra RL, and Volders PGA, "Noninvasive reconstruction of cardiac electrical activity: update on current methods, applications and challenges," Neth Heart J, vol. 23, no. 6, pp. 301-11, 2015.

[100] Erem B, Brooks DH, van Dam PM, Stinstra JG, and MacLeod RS, "Spatiotemporal estimation of activation times of fractionated ECGs on complex heart surfaces," Conf Proc IEEE Eng Med Biol Soc, vol. 2011, pp. 5884-7, 2011.

[101] Ghosh S and Rudy Y, "Accuracy of quadratic versus linear interpolation in noninvasive electrocardiographic imaging (ecgi)," Ann Biomed Eng, vol. 33, no. 9, pp. 1187-1201, 2005.

[102] Wang Y and Rudy Y, "Application of the method of fundamental solutions to potential-based inverse electrocardiography," Ann Biomed Eng, vol. 34, no. 8, pp. 1272-1288, 2006.

[103] Oster HS and Rudy Y, "Regional regularization of the electrocardiographic inverse problem: a model study using spherical geometry," IEEE Trans Biomed Eng, vol. 44, no. 2, pp. 188-99, 1997.

[104] Bear LR, Cheng LK, LeGrice IJ, Sands GB, Lever NA, Paterson DJ, and Smaill BH, "The forward problem of electrocardiography: is it solved?” Circ Arrhythm Electrophysiol, no. 8, pp. 677-684, 2015.

[105] The Maastricht ECGI Group. (2016), [Online]. Available: http://mecgi .org.

[106] Aras K, Good W, Tate J, Burton B, Brooks D, Coll-Font J, Doessel O, Schulze W, Potyagaylo D, Wang L, van Dam P, and MacLeod R, "Experimental data and geometric analysis repository-edgar," J Electrocardiol, vol. 48, no. 6, pp. 975-981, 2015.

[107] Van Oosterom A, "Closed-form analytical expressions for the potential fields generated by triangular monolayers with linearly distributed source strength," Medical \& Biological Engineering \& Computing, vol. 50, no. 1, pp. 1-9, 2011. 
[108] Pullan A, Cheng L, and Buist M, Mathematically modelling the electrical activity of the heart: from cell to body surface and back again. World Scientific, 2005.

[109] Cluitmans MJM, De Jong MMJ, Volders PGA, Peeters RLM, and Westra RL, "Physiologybased regularization improves noninvasive reconstruction and localization of cardiac electrical activity," in Computing in Cardiology, vol. 41, 2014, pp. $1-4$.

[110] Lopez-Rincon A, Bendahmane M, and Ainseba B, "On $3 \mathrm{~d}$ numerical inverse problems for the bidomain model in electrocardiology," Computers \& Mathematics with Applications, vol. 69, no. 4, pp. 255-74, 2015.

[111] Cluitmans MJM, Karel J, Bonizzi P, Jong M de, Volders P, Peeters R, and Westra RL, "Invivo evaluation of reduced-lead-systems in noninvasive reconstruction and localization of cardiac electrical activity," in Computing in Cardiology, vol. 42, 2015, pp. 221-4.

[112] Fitzhugh R, "Impulses and physiological states in theoretical models of nerve membrane," Biophys J, vol. 1, no. 6, pp. 445-66, 1961.

[113] Luo CH and Rudy Y, "A model of the ventricular cardiac action potential. depolarization, repolarization, and their interaction," Circ Res, vol. 68, no. 6, pp. 1501-26, 1991.

[114] Tusscher $\mathrm{KH}$ ten and Panfilov AV, "Alternans and spiral breakup in a human ventricular tissue model," American Journal of Physiology-Heart and Circulatory Physiology, vol. 291, no. 3, H1088-H1 100, 2006.

[115] Clerx M, Lange E de, Collins P, and Volders PGA, "Myokit: a simple interface to cardiac cellular electrophysiology," Progress in Biophysics and Molecular Biology, vol. 120, pp. 10014, 2016.

[116] Vandersickel N, Boer T de, Vos MA, and Panfilov AV, "Perpetuation of torsade de pointes in heterogeneous hearts: competing foci or re-entry?” Journal of Physiology, 2016.

[117] Tibshirani R, "Regression shrinkage and selection via the lasso," Journal of the Royal Statistical Society. Series B (Methodological), pp. 267-288, 1996.

[118] Ghosh S and Rudy Y, "Application of 11-norm regularization to epicardial potential solution of the inverse electrocardiography problem," Annals of Biomedical Engineering, vol. 37, no. 5, pp. 902-912, 2009.

[119] van Oosterom A, "The use of the spatial covariance in computing pericardial potentials," IEEE Trans Biomed Eng, vol. 46, no. 7, pp. 778-87, 1999.

[120] Geselowitz D and Miller W, "A bidomain model for anisotropic cardiac muscle," Annals of Biomedical Engineering, vol. 11, no. 3, pp. 191-206, 1983.

[121] Cluitmans M, Clerx M, Vandersickel N, Peeters R, Volders P, and Westra R, "Physiologybased regularization of the electrocardiographic inverse problem," In revision, 2016.

[122] Candes EJ, Wakin MB, and Boyd SP, "Enhancing sparsity by reweighted $\ell_{1}$ minimization," Journal of Fourier analysis and applications, vol. 14, no. 5-6, pp. 877-905, 2008.

[123] Zou H and Hastie T, "Regularization and variable selection via the elastic net," Journal of the Royal Statistical Society: Series B (Statistical Methodology), vol. 67, no. 2, pp. 301-320, 2005 .

[124] Hastie T, Tibshirani R, and Wainwright M, Statistical learning with sparsity: the lasso and generalizations. CRC Press, 2015.

[125] Qian J, Hastie T, Friedman J, Tibshirani R, and Simon N, Glmnet for matlab, 2013.

[126] Karel JMH, "A wavelet approach to cardiac signal processing for low-power hardware applications," PhD thesis, Maastricht University, 2009.

[127] Karel J, Peeters R, Westra R, Moermans K, Haddad S, and Serdijn W, "Optimal discrete wavelet design for cardiac signal processing," in Engineering in Medicine and Biology Society, 
2005. IEEE-EMBS 2005. 27th Annual International Conference of the, IEEE, 2005, pp. 27692772.

[128] Lee S, Zhu J, and Xing EP, “Adaptive multi-task lasso: with application to eqtl detection," in Advances in Neural Information Processing Systems 23, Lafferty J, Williams C, Shawe-Taylor J, Zemel R, and Culotta A, Eds., Curran Associates, Inc., 2010, pp. 1306-1314.

[129] Obozinski G, Taskar B, and Jordan M. (2006). Multi-task feature selection, [Online]. Available: http: // citeseerx . ist.psu . edu/viewdoc/download?doi=10 .1 1.1.94 . 951\& rep=repi\&type $=$ pdf.

[130] Erem B and Brooks DH, "Differential geometric approximation of the gradient and hessian on a triangulated manifold," Proc IEEE Int Symp Biomed Imaging, vol. 2011, pp. 504-507, 2011.

[131] Morita H, Wu J, and Zipes DP, “The qt syndromes: long and short," The Lancet, vol. 372, no. 9640, pp. 750-763, 2008.

[132] Baumert M, Porta A, Vos MA, Malik M, Couderc JP, Laguna P, Piccirillo G, Smith GL, Tereshchenko LG, and Volders PG, " $Q t$ interval variability in body surface ecg: measurement, physiological basis, and clinical value: position statement and consensus guidance endorsed by the european heart rhythm association jointly with the esc working group on cardiac cellular electrophysiology," Europace, euv405, 2016.

[133] Pelliccia A, Di Paolo FM, Quattrini FM, Basso C, Culasso F, Popoli G, De Luca R, Spataro A, Biffi A, Thiene G, et al., "Outcomes in athletes with marked ecg repolarization abnormalities," New England Journal of Medicine, vol. 358, no. 2, pp. 152-161, 2008.

[134] Sugrue A, Kremen V, Qiang B, Sheldon SH, DeSimone CV, Sapir Y, Striemer BL, Brady P, Asirvatham SJ, Ackerman MJ, Friedman P, and Noseworthy PA, "Electrocardiographic predictors of torsadogenic risk during dofetilide or sotalol initiation: utility of a novel t wave analysis program," Cardiovasc Drugs Ther, vol. 29, no. 5, pp. 433-441, 2015.

[135] Haïssaguerre M, Derval N, Sacher F, Jesel L, Deisenhofer I, Roy L de, Pasquié JL, Nogami A, Babuty D, Yli-Mayry S, et al., "Sudden cardiac arrest associated with early repolarization," New England Journal of Medicine, vol. 358, no. 19, pp. 2016-2023, 2008.

[136] Tikkanen JT, Anttonen O, Junttila MJ, Aro AL, Kerola T, Rissanen HA, Reunanen A, and Huikuri HV, "Long-term outcome associated with early repolarization on electrocardiography," New England Journal of Medicine, vol. 361, no. 26, pp. 2529-2537, 2009.

[137] Leenhardt A, Glaser E, Burguera M, Nürnberg M, Maison-Blanche P, and Coumel P, "Shortcoupled variant of torsade de pointes. a new electrocardiographic entity in the spectrum of idiopathic ventricular tachyarrhythmias," Circulation, vol. 89, no. 1, pp. 206-215, 1994.

[138] Heijman J, Spätjens RLHMG, Seyen SRM, Lentink V, Kuijpers HJH, Boulet IR, de Windt LJ, David M, and Volders PGA, "Dominant-negative control of camp-dependent iks upregulation in human long-qt syndrome type 1," Circ Res, vol. 110, no. 2, pp. 211-219, 2012.

[139] Higuchi $\mathrm{T}$ and Nakaya $\mathrm{Y}$, " $\mathrm{T}$ wave polarity related to the repolarization process of epicardial and endocardial ventricular surfaces," Am Heart J, vol. 108, no. 2, pp. 290-295, 1984.

[140] Coronel R, de Bakker JMT, Wilms-Schopman FJG, Opthof T, Linnenbank AC, Belterman $\mathrm{CN}$, and Janse $\mathrm{MJ}$, "Monophasic action potentials and activation recovery intervals as measures of ventricular action potential duration: experimental evidence to resolve some controversies," Heart Rhythm, vol. 3, no. 9, pp. 1043-1050, 2006.

[141] Striessnig J, Bolz HJ, and Koschak A, "Channelopathies in cav1.1, cav1.3, and cav1.4 voltage-gated l-type ca2+ channels,” Pflugers Arch, vol. 460, no. 2, pp. 361-374, 2010. 
[142] Mangoni ME, Couette B, Bourinet E, Platzer J, Reimer D, Striessnig J, and Nargeot J, "Functional role of l-type cav1.3 ca2+ channels in cardiac pacemaker activity," Proc Natl Acad Sci U S A, vol. 100, no. 9, pp. 5543-5548, 2003.

[143] Kruse M, Schulze-Bahr E, Corfield V, Beckmann A, Stallmeyer B, Kurtbay G, Ohmert I, Schulze-Bahr E, Brink P, and Pongs O, "Impaired endocytosis of the ion channel trpm4 is associated with human progressive familial heart block type i," J Clin Invest, vol. 1 19, no. 9, pp. 2737-2744, 2009.

[144] Guinamard R, Bouvagnet P, Hof T, Liu H, Simard C, and Sallé L, “Trpm4 in cardiac electrical activity," Cardiovasc Res, vol. 108, no. 1, pp. 21-30, 2015.

[145] Liu H, El Zein L, Kruse M, Guinamard R, Beckmann A, Bozio A, Kurtbay G, Mégarbané A, Ohmert I, Blaysat G, Villain E, Pongs O, and Bouvagnet P, "Gain-of-function mutations in trpm 4 cause autosomal dominant isolated cardiac conduction disease," Circ Cardiovasc Genet, vol. 3, no. 4, pp. 374-385, 2010.

[146] Liu H, Chatel S, Simard C, Syam N, Salle L, Probst V, Morel J, Millat G, Lopez M, Abriel H, Schott JJ, Guinamard R, and Bouvagnet P, "Molecular genetics and functional anomalies in a series of 248 brugada cases with 11 mutations in the trpm 4 channel," PLoS One, vol. 8, no. 1, e54131, 2013.

[147] Daumy X, Amarouch MY, Lindenbaum P, Bonnaud S, Charpentier E, Bianchi B, Nafzger S, Baron E, Fouchard S, Thollet A, Kyndt F, Barc J, Le Scouarnec S, Makita N, Le Marec H, Dina C, Gourraud JB, Probst V, Abriel H, Redon R, and Schott JJ, "Targeted resequencing identifies trpm 4 as a major gene predisposing to progressive familial heart block type i," Int J Cardiol, vol. 207, pp. 349-358, 2016.

[148] Ullrich ND, Voets T, Prenen J, Vennekens R, Talavera K, Droogmans G, and Nilius B, “Comparison of functional properties of the ca2+-activated cation channels trpm 4 and trpm 5 from mice," Cell Calcium, vol. 37, no. 3, pp. 267-278, 2005.

[149] Nilius B, Prenen J, Droogmans G, Voets T, Vennekens R, Freichel M, Wissenbach U, and Flockerzi V, "Voltage dependence of the ca2+-activated cation channel trpm4," J Biol Chem, vol. 278, no. 33, pp. 30813-30820, 2003.

[150] Courtemanche M, Ramirez RJ, and Nattel S, "Ionic mechanisms underlying human atrial action potential properties: insights from a mathematical model," Am J Physiol, vol. 275, no. 1 Pt 2, $\mathrm{H}_{3} 01-\mathrm{H}_{3} 21,1998$.

[151] Stewart P, Aslanidi OV, Noble D, Noble PJ, Boyett MR, and Zhang H, "Mathematical models of the electrical action potential of purkinje fibre cells," Philos Trans A Math Phys Eng Sci, vol. 367 , no. 1896, pp. 2225-2255, 2009.

[152] O'Hara T, Virág L, Varró A, and Rudy Y, "Simulation of the undiseased human cardiac ventricular action potential: model formulation and experimental validation," PLoS Comput Biol, vol. 7, no. 5, e1002061, 2011.

[153] Molina C, Heijman J, and Dobrev D, "Differences in left versus right ventricular electrophysiological properties in cardiac dysfunction and arrhythmogenesis," Arrhythmia \& Electrophysiology Review, vol. 5, no. 1, pp. 14-9, 2016.

[154] Zhang J, Sacher F, Hoffmayer K, O'Hara T, Strom M, Cuculich P, Silva J, Cooper D, Faddis M, Hocini M, Haïssaguerre M, Scheinman M, and Rudy Y, "Cardiac electrophysiological substrate underlying the ecg phenotype and electrogram abnormalities in brugada syndrome patients," Circulation, vol. 131, no. 22, pp. 1950-1959, 2015.

[155] Slawsky MT and Castle NA, "K+ channel blocking actions of flecainide compared with those of propafenone and quinidine in adult rat ventricular myocytes," J Pharmacol Exp Ther, vol. 269, no. 1, pp. 66-74, 1994. 
[156] Yang T, Snyders DJ, and Roden DM, "Inhibition of cardiac potassium currents by the vesnarinone analog opc-18790: comparison with quinidine and dofetilide," J Pharmacol Exp Ther, vol. 280, no. 3, pp. 1170-1175, 1997.

[157] Hill RJ, Duff HJ, and Sheldon RS, "Class i antiarrhythmic drug receptor: biochemical evidence for state-dependent interaction with quinidine and lidocaine," Mol Pharmacol, vol. 36, no. 1, pp. 150-159, 1989 .

[158] Wang Z, Fermini B, and Nattel S, "Effects of flecainide, quinidine, and 4-aminopyridine on transient outward and ultrarapid delayed rectifier currents in human atrial myocytes," $J$ Pharmacol Exp Ther, vol. 272, no. 1, pp. 184-196, 1995.

[159] Punshchykova O, Švehlíková J, Tyšler M, Grünes R, Sedova K, Osmančík P, Ždárská J, Heřman D, and Kneppo P, "Influence of torso model complexity on the noninvasive localization of ectopic ventricular activity," Measurement Science Review, vol. 16, no. 2, pp. 96-102, 2016.

[160] Bear LR, Smaill BH, LeGrice IJ, Sands GB, Lever NA, Paterson DJ, and Cheng LK, "How accurate is inverse electrocardiograpic mapping? a systematic evaluation in pigs," Personal communication (2015).

[161] Consortium for ECG Imaging Workgoup on PVC Localization. (). 2016, [Online]. Available: http://www.ecg-imaging.org/workgroups/pvc-localization.

[162] Erem B, Coll-Font J, Orellana RM, Stovícek P, and Brooks DH, "Using transmural regularization and dynamic modeling for noninvasive cardiac potential imaging of endocardial pacing with imprecise thoracic geometry," IEEE Trans Med Imaging, vol. 33, no. 3, pp. $726-$ $738,2014$.

[163] Gordon JP, Laks M, Boyle NG, and Dam PM van, "Advantages of the cardiac isochrone positioning system for localization of pvcs to the endocardium, epicardium, and midmyocardium from a 12 lead ecg," Circulation, vol. 130, no. Suppl 2, A19734-A19734, 2014.

[164] Van Dam PM, Oostendorp TF, Linnenbank AC, and Van Oosterom A, "Non-invasive imaging of cardiac activation and recovery," Annals of Biomedical Engineering, vol. 37, no. 9, pp. 1739-56, 2009.

[165] Zeemering S, "Sparse estimation: applications in atrial fibrillation," $\mathrm{PhD}$ thesis, Maastricht University, 2015.

[166] Eugenio PL, "Frequent premature ventricular contractions: an electrical link to cardiomyopathy," Cardiol Rev, vol. 23, no. 4, pp. 168-172, 2015.

[167] Latchamsetty R and Bogun F, "Premature ventricular complexes and premature ventricular complex induced cardiomyopathy," Curr Probl Cardiol, vol. 40, no. 9, pp. 379-422, 2015.

[168] Bogun F, Crawford T, Reich S, Koelling TM, Armstrong W, Good E, Jongnarangsin K, Marine JE, Chugh A, Pelosi F, Oral H, and Morady F, "Radiofrequency ablation of frequent, idiopathic premature ventricular complexes: comparison with a control group without intervention," Heart Rhythm, vol. 4, no. 7, pp. 863-867, 2007.

[169] Haïssaguerre M, Extramiana F, Hocini M, Cauchemez B, Jaïs P, Cabrera JA, Farré J, Farre G, Leenhardt A, Sanders P, Scavée C, Hsu LF, Weerasooriya R, Shah DC, Frank R, Maury $\mathrm{P}$, Delay M, Garrigue $\mathrm{S}$, and Clémenty J, "Mapping and ablation of ventricular fibrillation associated with long-qt and brugada syndromes," Circulation, vol. 108, no. 8, pp. 925-928, 2003.

[170] Nam GB, Ko KH, Kim J, Park KM, Rhee KS, Choi KJ, Kim YH, and Antzelevitch C, "Mode of onset of ventricular fibrillation in patients with early repolarization pattern vs. brugada syndrome," Eur Heart J, vol. 31, no. 3, pp. 330-339, 2010. 
[171] Viskin S, Lesh MD, Eldar M, Fish R, Setbon I, Laniado S, and Belhassen B, "Mode of onset of malignant ventricular arrhythmias in idiopathic ventricular fibrillation," $J$ Cardiovasc Electrophysiol, vol. 8, no. 10, pp. 1115-1120, 1997.

[172] Bänsch D, Oyang F, Antz M, Arentz T, Weber R, Val-Mejias JE, Ernst S, and Kuck KH, "Successful catheter ablation of electrical storm after myocardial infarction," Circulation, vol. 108, no. 24, pp. 3011-3016, 2003.

[173] Haïssaguerre M, Shoda M, Jaïs P, Nogami A, Shah DC, Kautzner J, Arentz T, Kalushe D, Lamaison D, Griffith M, Cruz F, de Paola A, Gaïta F, Hocini M, Garrigue S, Macle L, Weerasooriya R, and Clémenty J, "Mapping and ablation of idiopathic ventricular fibrillation," Circulation, vol. 106, no. 8, pp. 962-967, 2002.

[174] Abdalla IS, Prineas RJ, Neaton JD, Jacobs Jr D, and Crow RS, "Relation between ventricular premature complexes and sudden cardiac death in apparently healthy men," Am J Cardiol, vol. 6o, no. 13, pp. 1036-1042, 1987.

[175] Lang RM, Badano LP, Mor-Avi V, Afilalo J, Armstrong A, Ernande L, Flachskampf FA, Foster E, Goldstein SA, Kuznetsova T, Lancellotti P, Muraru D, Picard MH, Rietzschel ER, Rudski L, Spencer KT, Tsang W, and Voigt JU, "Recommendations for cardiac chamber quantification by echocardiography in adults: an update from the american society of echocardiography and the european association of cardiovascular imaging," Eur Heart J Cardiovasc Imaging, vol. 16, no. 3, pp. 233-270, 2015.

[176] Cuculich P, Schill M, Kashani R, Cooper DH, Faddis MN, Gleva MJ, Noheria A, Smith $\mathrm{TW}$, and Robinson C, "First report of entirely noninvasive stereotactic cardiac ablation radiotherapy (no-scar) for vt in humans," Heart Rhythm, vol. 13, no. 5, S $S_{13} 8,2016$.

[177] Erkapic D and Neumann T, "Ablation of premature ventricular complexes exclusively guided by three-dimensional noninvasive mapping," Cardiac Electrophysiology Clinics, vol. 7, no. 1, 109 â€“"115, 2015.

[178] Erkapic D, Greiss H, Pajitnev D, Zaltsberg S, Deubner N, Berkowitsch A, Möllman S, Sperzel J, Rolf A, Schmitt J, Hamm CW, Kuniss M, and Neumann T, "Clinical impact of a novel three-dimensional electrocardiographic imaging for non-invasive mapping of ventricular arrhythmias-a prospective randomized trial," Europace, vol. 17, no. 4, pp. 591-597, 2015 .

[179] Wissner E, Revishvili A, Metzner A, Tsyganov A, Kalinin V, Lemes C, Saguner AM, Maurer T, Deiss S, Sopov O, Labarkava E, Chmelevsky M, and Kuck KH, "Noninvasive epicardial and endocardial mapping of premature ventricular contractions," Europace, 2016.

[180] van Dam PM, Gordon JP, Laks MM, and Boyle NG, "Development of new anatomy reconstruction software to localize cardiac isochrones to the cardiac surface from the 12 lead ecg," J Electrocardiol, vol. 48, no. 6, 959-965, 2015.

[181] Cochet H, Dubois R, Sacher F, Derval N, Sermesant M, Hocini M, Montaudon M, Haïssaguerre $\mathrm{M}$, Laurent $\mathrm{F}$, and Jaïs $\mathrm{P}$, "Cardiac arrythmias: multimodal assessment integrating body surface ecg mapping into cardiac imaging,” Radiology, vol. 271, no. 1, pp. 239-247, 2014.

[182] Yamashita S, Sacher F, Mahida S, Berte B, Lim HS, Komatsu Y, Amraoui S, Denis A, Derval N, Laurent F, Montaudon M, Hocini M, Haïssaguerre M, Jaïs P, and Cochet H, "Role of high-resolution image integration to visualize left phrenic nerve and coronary arteries during epicardial ventricular tachycardia ablation," Circ Arrhythm Electrophysiol, vol. 8, no. 2, pp. 371-380, 2015.

[183] Yamashita S, Sacher F, Mahida S, Berte B, Lim HS, Komatsu Y, Amraoui S, Denis A, Derval N, Laurent F, Sermesant M, Montaudon M, Hocini M, Haïssaguerre M, Jaïs P, and Cochet 
$\mathrm{H}$, "Image integration to guide catheter ablation in scar-related ventricular tachycardia," $J$ Cardiovasc Electrophysiol, vol. 27, no. 6, 699-708, 2016.

[184] Wellens HJJ, “Cardiac arrhythmias: the quest for a cure: a historical perspective," J Am Coll Cardiol, vol. 44, no. 6, pp. 1155-1163, 2004.

[185] van der Werf C, van Langen IM, and Wilde AAM, "Sudden death in the young: what do we know about it and how to prevent?" Circ Arrhythm Electrophysiol, vol. 3, no. 1, pp. 96-104, 2010.

[186] Mozaffarian D, Benjamin EJ, Go AS, Arnett DK, Blaha MJ, Cushman M, de Ferranti S, Després JP, Fullerton HJ, Howard VJ, Huffman MD, Judd SE, Kissela BM, Lackland DT, Lichtman JH, Lisabeth LD, Liu S, Mackey RH, Matchar DB, McGuire DK, Mohler 3rd ER, Moy CS, Muntner P, Mussolino ME, Nasir K, Neumar RW, Nichol G, Palaniappan L, Pandey DK, Reeves MJ, Rodriguez CJ, Sorlie PD, Stein J, Towfighi A, Turan TN, Virani SS, Willey JZ, Woo D, Yeh RW, Turner MB, AHASC, and Subcommittee SS, "Heart disease and stroke statistics-2015 update: a report from the american heart association," Circulation, vol. 131, no. 4, e29-322, 2015.

[187] Hoebe C, Cluitmans J, Wagenvoort J, Van Leeuwen W, and Bilkert-Mooiman M, "Koud leidingwater als bron van fatale nosocomiale pneumonieën door legionella pneumophila in een revalidatiekliniek," Nederlands tijdschrift voor geneeskunde, vol. 143, no. 20, pp. 1041$1045,1999$.

[188] Knollmann BC and Roden DM, "A genetic framework for improving arrhythmia therapy," Nature, vol. 451, no. 7181, pp. 929-936, 2008.

[189] Liu M, Yang KC, and Dudley Jr SC, "Cardiac sodium channel mutations: why so many phenotypes?” Nat Rev Cardiol, vol. 11, no. 10, pp. 607-615, 2014. 